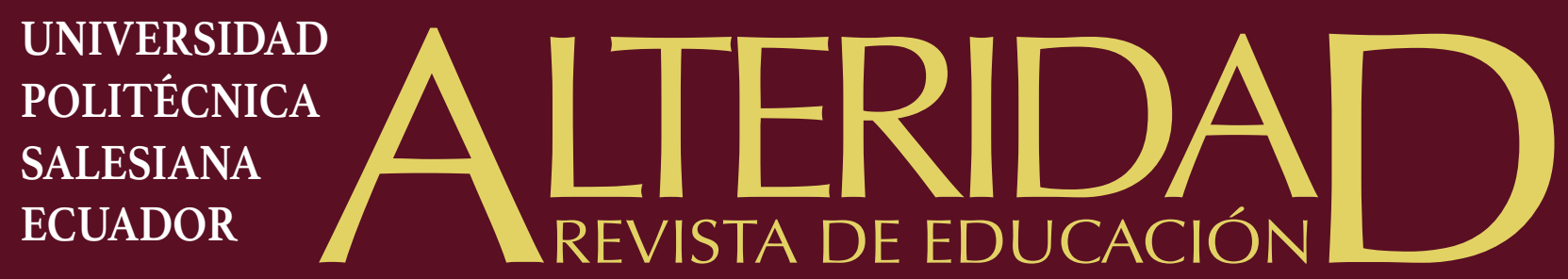

ISSN impreso I390-325X

Período enero - junio de 2022

ISSN electrónico I390-8642

Vol. I7, No. I

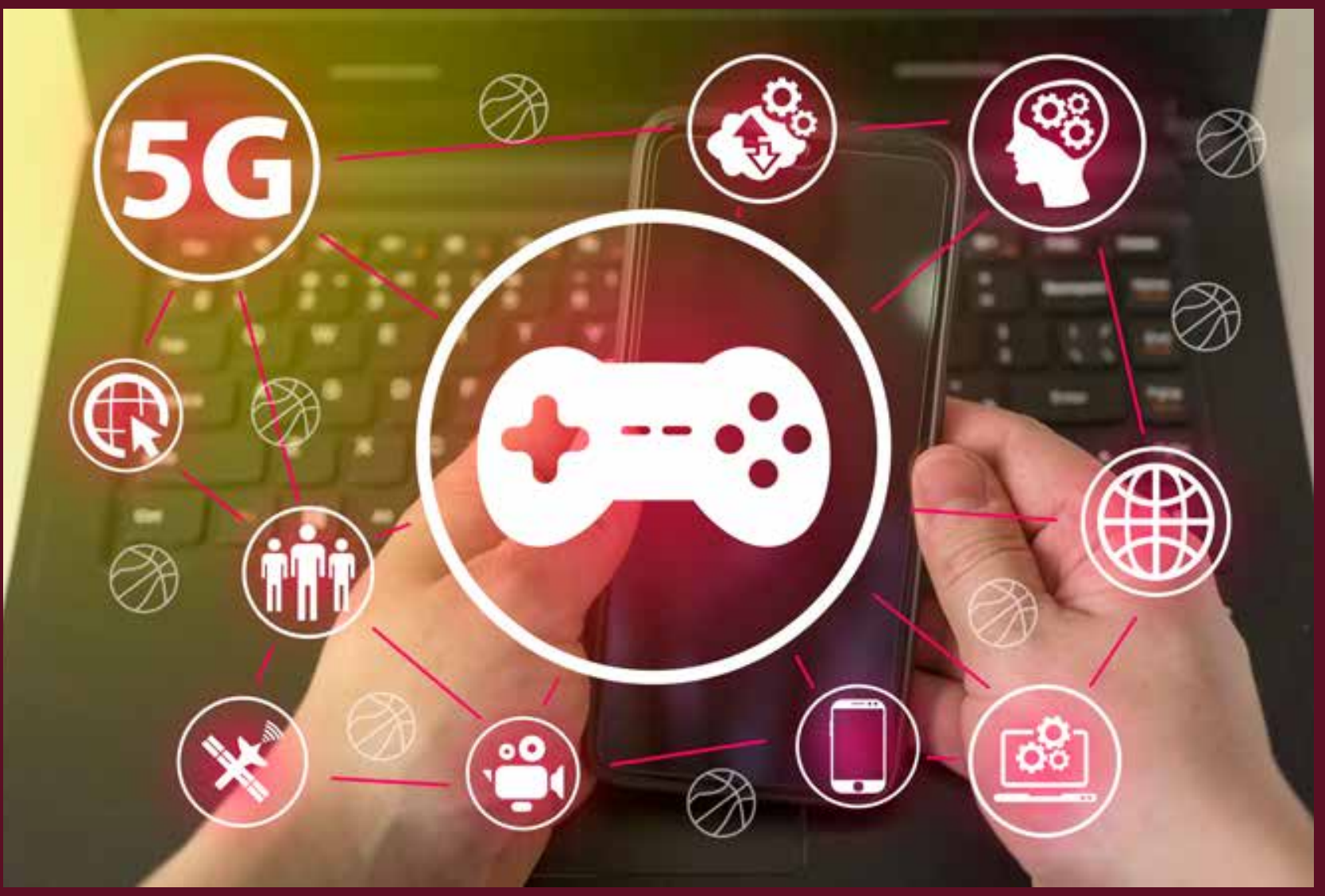

La gamificación en el aprendizaje: Estrategia metodológica para la motivación del alumnado

"Gamification in learning: a methodological strategy to motivate students" 


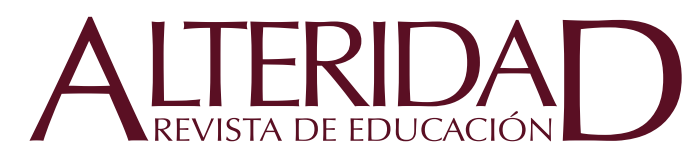

Volumen I7, número I (Período enero - junio 2022)

\section{Indexaciones}

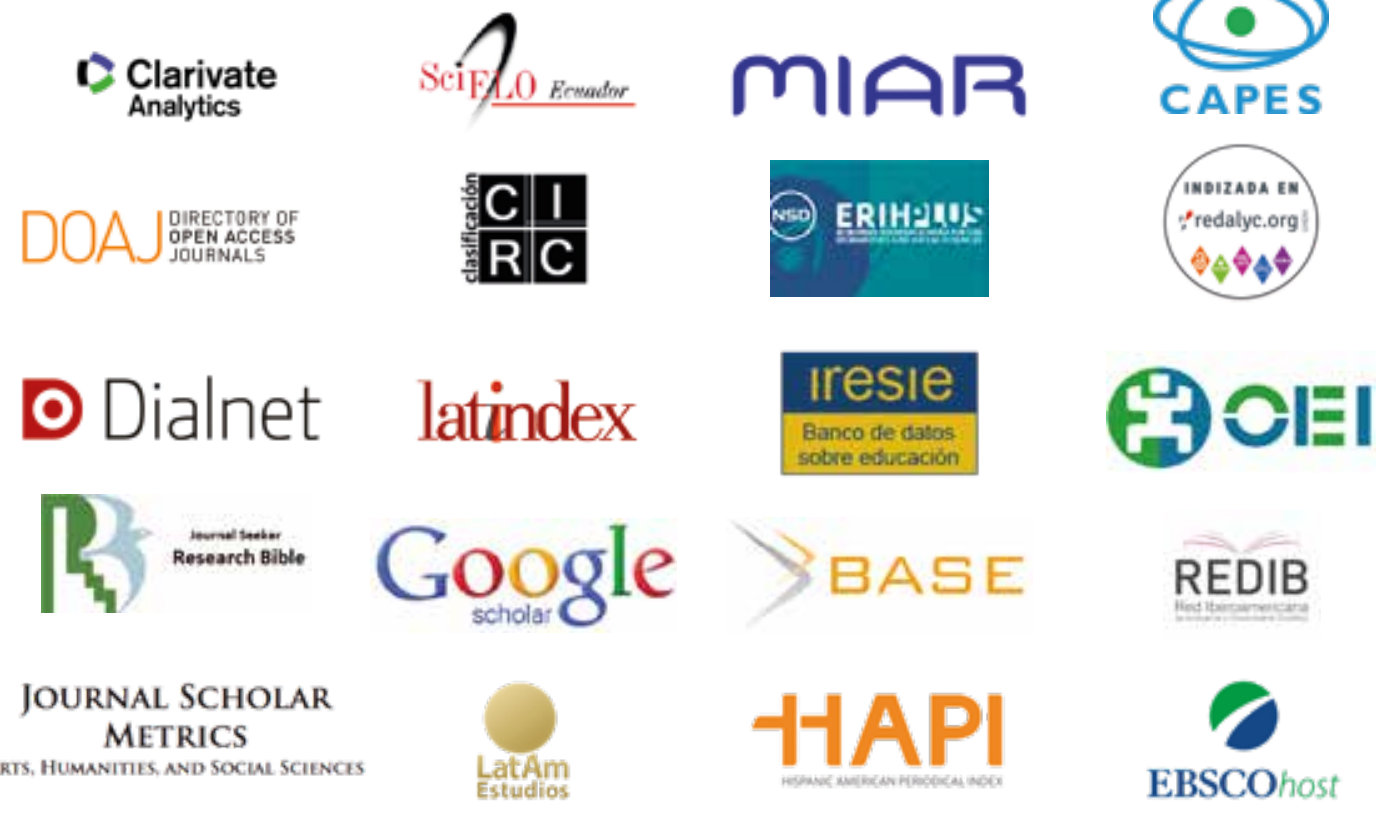

\section{Criterios de calidad del proceso editorial}

Crossref

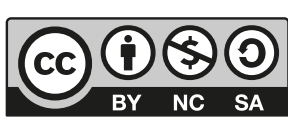

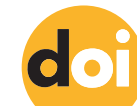

SHERPA/RøMEO

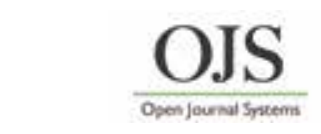

turniting)

\section{Revistas consorciadas}

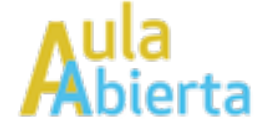

ENSEÑ ANZA

Teaching

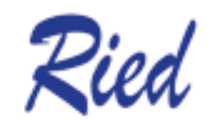

at
@

RECIE edmetic 


\section{Consejo de Editores (Editors Board)}

Editores Jefe (Editor-in-Chief)

- Dr. Jaime Padilla-Verdugo, Editor Jefe. Universidad

Politécnica Salesiana (Ecuador).

- Dra. Verónica Marín-Díaz, Editora Jefe. Universidad de Córdova (España).

Editores Asociados (Associate Editors)

- Dra. Victoria Jara-Cobos, Editora Asociada. Universidad Politécnica Salesiana (Ecuador).

Editores temáticos (Thematic Editors)

- Dra. Carmen Llorente-Cejudo, Universidad de Sevilla (España)

- Dr. Javier Gil-Quintana, Universidad Nacional de Educación a Distancia (España)

- Dra. Alejandra Hurtado Mazeyra, Universidad Nacional de San Agustín (Perú)

Co-Editores internacionales (International Coeditors)

Dr. Geraldo Caliman, Universidade Católica de Brasília, (Brasil)

\section{Consejo Científico (Advisory Board)}

- Dr. Alejandro Rodríguez-Martín, Universidad de Oviedo, España

- Dr. Alfonso Torres Carrillo, Universidad Pedagógica Nacional, Colombia

- Dr. Alfredo Veiga-Neto, Universidade Federal do Rio Grande do Sul, Brasil

- Dra. Ana Castro Zubizarreta, Universidad de Cantabria, España

- Dra. Ana Rosa Arias Gago, Universidad de León, España

- Dr. Antonio Bautista García-Vera, Universidad Complutense de Madrid, España

- Dra. Armanda Matos, Universidad de Coímbra, Portugal

- Dr. Carlos Paladines Escudero, Pontificia Universidad Católica del Ecuador, Ecuador

- Dra. Carmen Martínez Novo, Universidad de Florida, USA

- Dra. Cristina Allemann-Ghionda, Universidad de Colonia, Alemania

- Dr. Daniel Mato, Universidad Nacional Tres de Febrero, Argentina

- Dra. Diana Rivera Rogel, Universidad Técnica Particular de Loja, Ecuador

- Dr. Emilio Álvarez-Arregui, Universidad de Oviedo, España

- Dr. Felipe Trillo Alonso, Universidad de Santiago de Compostela, España

- Dra. Gabriela Borjes, Universidade Federal de Juiz de Fora, Brasil

- Dr. Geraldo Caliman, Universidade Católica de Brasília, Brasil

- Dr. Gunther Dietz, Universidad Veracruzana, México

- Dr. Ignacio Aguaded, Universidad de Huelva, España

- Dr. Jairo Lugo-Ocando, Northwestern University in Qatar, Qatar

- Dr. Javier Loredo Enríquez, Universidad Iberoamericana, México

- Dr. Jesús María Salinas Ibáñez, Universidad de las Islas Baleares, España

- Dr. Jorge Maldonado Mahauad, Universidad de Cuenca, Ecuador

- Dr. José Antonio Caride Gómez, Universidad de Santiago de Compostela, España

- Dr. José Joaquín Brunner, Universidad Diego Portales, Chile

- Dr. José Manuel Castellano Gil, Universidad Nacional de Educación, Ecuador

- Dr. José Tejada Fernández, Universidad Autónoma de Barcelona, España

- Dr. Juan Silva Quiroz, Universidad de Santiago de Chile, Chile

- Dr. Julio Cabero-Almenara, Universidad de Sevilla, España

- Dr. Lorenzo Cantoni, Università della Svizzera Italiana, Suiza
- Dr. Luis Miguel Pedrero Esteban, Universidad Pontificia de Salamanca, España

- Dr. Luis Miguel Romero Rodríguez, Universidad Internacional de La Rioja, España

- Dr. Manuel Area, Universidad de La Laguna, España

- Dra. María Caridad Valdés Rodríguez, Universidad de las Ciencias Informáticas, Cuba

- Dra. Maria Isabel Punín Larrea, Universidad Técnica Particular de Loja, Ecuador

- Dra. María Teresa Bejarano Franco, Universidad de Castilla La Mancha, España

- Dra. Maria-Soledad Ramírez-Montoya, TEC de Monterrey, México

- Dra. Marina Isabel Pagani, Universidad de Concepción del Uruguay, Argentina

- Dr. Maurice Tardif, Université de Montréal, Canadá

- Dra. Mercedes González-Sanmamed, Universidad de La Coruña, España

- Dra. Natalia González Fernández, Universidad de Cantabria, España

- Dra. Paloma Antón Ares, Universidad Complutense de Madrid, España

- Dra. Paula Renés Arellano, Universidad de Cantabria, España

- Dr. Peter McLaren, Chapman University, USA

- Dra. Pilar Arnáiz Sánchez, Universidad de Murcia, España

- Dr. Rafael Tejeda Díaz, Universidad Técnica de Manabí, Ecuador

- Dr. Ronaldo Zacharias, Centro Universitário Salesiano de São Paulo, Brasil

- Dra. Rosa García-Ruiz, Universidad de Cantabria, España

- Dra. Soraya Ferreira Vieira, Universidade Federal de Juiz de Fora, Brasil

- Dra. Sylvia Schmelkes del Valle, Universidad Iberoamericana, México

- Dra. Verónica Marín Díaz, Universidad de Córdova, España

- Dr. Vicent Gozálvez Pérez, Universidad de Valencia, España

Consejo Internacional de Revisores (International Board of Reviewers)

- Dra. Adelina de Oliveira Novaes, Universidade Cidade de São Paulo, Brasil

- Dra. Adir Casaro Nascimento, Universidade Catolica Dom Bosco, Brasil

- Dr. Adrián Segura-Robles, Universidad de Granada, España

- Dra. Adriana Gewerc, Universidad de Santiago de Compostela, España

- Dra. Adriana Peña Pérez Negrón, Universidad de Guadalajara, México

- Dra. Águeda Delgado-Ponce, Universidad de Huelva, España

- Dra. Alba García Barrera, Universidad a Distancia de Madrid, España

- Dr. Alberto Ayaviri-Panozo, Universidad Mayor Real, Bolivia

- Dr. Alejandro Escudero-Santana, Universidad de Sevilla, España

- Dr. Alejandro Marquez Jiménez, IISUE, México

- Dra. Aleksandra Jablonska, Universidad Pedagógica Nacional, México

- Dra. Alessandra Diehl, Universidade de Ribeirão Preto, Brasil

- Dra. Alexandra Santamaría-Urbieta, Universidad Internacional de La Rioja, España

- Dr. Alexandre Godoy Dotta,UniBrasil Centro Universitário, Brasil

- Dr. Alexandre Martins, Marquette University, USA 
- Dra. Alicia Inés Villa, Universidad Nacional de La Plata, Argentina

- Dra. Alma Rosa Hernández Mondragón, Universidad La Salle, México

- Álvaro Guaymás, Universidad Nacional de Salta (UNSa), Argentina

- Dr. Amauris Laurencio Leyva, Universidad de La Habana, Cuba

- Dra. Amelia Gort Almeida, Universidad de La Habana, Cuba

- Dra. Amneris Chaparro Martínez, UNAM, México

- Dra. Ana Atorresi, Universidad Nacional de Río Negro, Argentina

- Dra. Ana Carolina Maldonado-Fuentes, Universidad del Bío-Bío, Chile

- Ana Cristina Canosa, Centro Universitário Salesiano de São Paulo, Brasil

- Dra. Ana Isabel González Contreras, Universidad de Extremadura, España

- Dra. Ana Lucia Braz, Universidade do Grande ABC, Brasil

- Dra. Ana Lupita Chaves, Universidad de Costa Rica, Costa Rica

- Ana Marcela Castellanos Guzmán, Universidad La Salle, México

- Dra. Ana María Barbancho, Universidad de Málaga, España

- Dra. Ana Maria Eyng, PUCPR, Brasil

- Dra. Ana Pérez-Escoda, Universidad Internacional de La Rioja, España

- Dr. André Boccato de Almeida, PUC-SP, Brasil

- Dra. Ángela Santamaría Chavarro, Universidad del Rosario, Colombia

- Dra. Angélica Guzmán Droguett, Pontificia Universidad Católica de Chile, Chile

- Dra. Anita Gramigna, Università degli Studi di Ferrara, Italia

- Dra. Annachiara Del Prete, Pontificia Universidad Católica de Valparaíso, Chile

- Anne Köster, European University Viadrina, Alemania

- Dra. Antonella Cagnolati, Università degli Studi di Foggia, Italia

- Dra. Antonia Ramírez-García, Universidad de Córdova, España

- Dr. Antonio Hilario Aguilera Urquiza, UFMS, Brasil

- Dr. Antônio Igo Barreto Pereira, Universidade Federal do Acre, Brasil

- Dr. Antonio Matas-Terrón, Universidad de Málaga, España

- Dr. Aristeo Santos López, UAEM, México

- Dra. Azucena Ochoa Cervantes, Universidad Autónoma de Querétaro, México

- Dra. Beatriz Gualdieri, Universidad Nacional de Luján, Argentina

- Dra. Bianca Thoilliez Ruano, Universidad Autónoma de Madrid, España

- Dr. Blas Orlando Garzón Vera, Universidad Politécnica Salesiana, Ecuador

- Dra. Carina Fracchia, Universidad Nacional del Comahue, Argentina

- Dra. Carla Förster Marín, Pontificia Universidad Católica de Chile, Chile

- Dr. Carlos Roberto Ruano, University of Toronto, Canadá

- Dra. Carmen Cantillo Valero, Universidad Nacional de Educación a Distancia, España

- Dra. Carmen Marta-Lazo, Universidad de Zaragoza, España

- Dra. Cecilia Dolores Correa de Molina, Universidad Simón Bolívar, Colombia

- Dra. Chantal-M Biencinto-López, Universidad Complutense de Madrid, España

- Dra. Claudia Araújo de Lima, Universidade Federal de Mato Grosso do Sul, Brasil

- Dra. Claudia Vélez De La Calle, Universidad de San Buenaventura de Cali, Colombia

- Dra. Claudia Moreno Standen, Universidad de Chile, Chile

- Dra. Constantina Xavier Filha, Universidade Federal de Mato Grosso do Sul, Brasil

- Dra. Damia Mendoza-Zambrano, Universidad Laica Eloy Alfaro de Manabí, Ecuador

- Dr. Daniel Leonidas Loncón, UNPSJB, Argentina

- Dr. Daniel Llanos Erazo, Universidad Politécnica Salesiana, Ecuador
- Dra. Daniela Maturana Castillo, Universidad de Santiago de Chile, Chile

- Dr. David Ruiz Torres, Universidad de Granada, España

- Diana Ávila, Universidad Politécnica Salesiana, Ecuador

- Doris Macías Mendoza, Universitat Politècnica de València, España

- Dr. Eduardo Amadeu Dutra Moresi, Universidade de Brasilia, Brasil

- Dr. Eduardo Fabara Garzón, Universidad Andina "Simón Bolívar", Ecuador

- Dr. Eduardo Mercado Cruz, ISCEEM, México

- Dr. Edwin Fabián Bersosa Vaca, Universidad Politécnica Salesiana, Ecuador

- Dra. Elena Guadalupe Rodríguez-Roa, Universidad La Salle, México

- Dra. Elisabete Oliveira, ECOS - Comunicação em Sexualidade, Brasil

- Dra. Elsa Celina Gatti García, Universidad de la República, Uruguay

- Dra. Emiko Saldivar, University of California - Santa Barbara, USA

- Dr. Emiliano Gil Blanco, Universidad San Francisco de Quito, Ecuador

- Dr. Enrique Arturo Parra Marín, Universidad de Chile, Chile

- Dr. Enrique Sánchez Rivas, Universidad de Málaga, España

- Dr. Eric Passone, Universidade Cidade de São Paulo, Brasil

- Dr. Ernesto Colomo-Magaña, Universidad de Málaga, España

- Dr. Ernesto Guerra García, Universidad Autónoma Indígena de México, México

- Dra. Eska Elena Solano Meneses, UAEM, México

- Dr. Felipe de Jesús Perales Mejía, UPN - Unidad Torreón, México

- Dr. Fernando Lara, Pontificia Universidad Católica del Ecuador, Ecuador

- Fernando Paladines, Universidad Politécnica Salesiana, Ecuador

- Dra. Flor Marina Bermúdez-Urbina, UNICACH, México

- Dr. Francisco Enrique Rodríguez-Alveal, Universidad del BíoBío, Chile

- Dr. Francisco Javier Merchán Iglesias, Universidad de Sevilla, España

- Dr. Francisco Jiménez Jiménez, Universidad de La Laguna, España

- Dr. Francisco José Fernández-Cruz, Universidad Complutense de Madrid, España

- Dra. Gema Sáez Rodríguez, Universidad Francisco de Vitoria, España

- Dr. Gersem dos Santos Luciano, Universidade Federal do Amazonas, Brasil

- Gianella Carrión-Salinas, Universidad Técnica Particular de Loja, Ecuador

- Dra. Gina Benavides Llerena, Universidad Andina “Simón Bolívar", Ecuador

- Giovanna Barroca de Moura, Universidade Estadual dal Paraíba, Brasil

- Dra. Gloria Piedad Gasca-Hurtado, Universidad de Medellín, Colombia

- Dr. Greby Uriel Rioja Montaño, Universidad Mayor de San Simón, Bolivia

- Dr. Helio Aisenberg Ferenhof, Universidade Federal de Santa Catarina, Brasil

- Dr. Henry Chero Valdiviezo, Universidad Católica Los Ángeles de Chimbote, Perú

- Dra. Herminia Hernández, Universidad de La Habana, Cuba

- Dr. Hugues Ribeiro, Universidade Estadual Paulista, Brasil

- Dra. Irene García Lázaro, Universidad de Sevilla, España

- Dra. Irene Melgarejo Moreno, Universidad Católica de Murcia, España

- Dra. Irina Salcines Talledo, Universidad de Cantabria, España

- Dra. Isabel-Maria Gallardo Fernández, Universitat de Valencia España 
- Dra. Isabel María Gómez Trigueros, Universidad de Alicante, España

- Dra. Isabel Sonsoles De Soto García, Universidad Pública de Navarra, España

- Dr. Ivan Sanchez Soto, Universidad del Bio Bio, Chile

- Dr. Ivar César Oliveira de Vasconcelos, Universidade Católica de Brasília, Brasil

- Dr. Jaime Rivas Castillo, Universidad Don Bosco, El Salvador

- Dr. Javier Gil-Quintana, Universidad Nacional de Educación a Distancia, España

- Dr. Jean Carlos García Zacarías, PUCE, Ecuador

- Dra. Jennie Brand Barajas, Universidad La Salle, México

- Dra. Jessica Paños Castro, Universidad de Deusto, España

- Dr. Jesús Cabrerizo Diago, UNED, España

- Dr. Jesus Fernández Gavira, Universidad de Sevilla, España

- Dr. Jorge Agustín Zapatero, Universidad Complutense de Madrid, España

- Dr. Jorge Leoncio Rivera Muñoz, Universidad Nacional Mayor de San Marcos, Perú

- Dr. José Alfonso Jiménez Moreno, UABC, México

- Dr. José Andrés García Méndez, ENAH, México

- Dr. José Antonio Álvarez Bermejo, Universidad de Almeria, España

- Dr. José Antonio Fernández Bravo, Universidad Camilo José Cela, España

- Dr. José Antonio Marín Marín, Universidad de Granada, España

- Dr. José Antonio Trasferetti, Pontifícia Universidade Católica de Campinas, Brasil

- Dr. José Francisco Alvarado García, Universidad Iberoamericana, México

- Dr. José Guilherme dos Santos Fernandes, Universidade Federal do Pará, Brasil

- Dr. José Luis Guzón Nestar, CES Don Bosco, UCM y San Pío X, UPSA, España

- Dr. José Luis Moral, Università Pontificia Salesiana, Italia

- Dr. José Manuel Meza Cano, Universidad Nacional Autonoma de Mexico, México

- Dr. José Raúl Jiménez Ibáñez, Universidad de La Salle, Colombia

- Dra. Josiane Peres Gonçalves, Universidade Federal de Mato Grosso do Sul, Brasil

- Dr. Josu Ahedo, Universidad Internacional de La Rioja, España

- Dr. Juan José Leiva Olivencia, Universidad de Málaga, España

- Dr. Juan Vicente Ortiz Franco, Fundación Universitaria Los Libertadores, Colombia

- Dra. Juana Yadira Martín Perico, USTA, España

- Dra. Kajiana Nuernberg Sartor Vidotto, UFSC, Brasil

- Dra. Kathie Njaine, Fundação Oswaldo Cruz, Brasil

- Laura Liliana Rosso, Universidad Nacional de Nordeste, Argentina

- Dra. Laura Sánchez Blanco, Universidad Pontificia de Salamanca, España

- Dra. Laura Selene Mateos-Cortés, Universidad Veracruzana, México

- Dr. Leonardo J. Ramirez, Universidad Militar de Nueva Granada, Colombia

- Dra. Leonor Buendía Eisman, Universidad de Granada, España

- Dra. Liliana Inés Ávila Garzón, UPTC, Colombia

- Dra. Liliana Mayer, UMET, Argentina

- Dra. Lisset Coba Mejía, Facultad Latinoamericana de Ciencias Sociales, Ecuador

- Dra. Lorena Pastor Gil, Universidad Complutense de Madrid, España

- Dr. Luis Antonio Reyes Ochoa, Universidad Católica Silva Henríquez, Chile

- Dr. Luis Enrique Sime Poma, Pontificia Universidad Católica del Perú, Perú

- Dr. Luis Fernando Cuji Llugna, Universidad Nacional de Tres de Febrero, Argentina
- Dr. Luis Fernando García Álvarez, INAH, México

- Dr. Luis Huerta-Charles, New Mexico State University, USA

- Dra. Ma.Dolores Díaz Noguera, Universidad de Sevilla, España

- Dra. Mara Lúcia Castilho, Instituto Federal de Brasilia, Brasil

- Dra. Marcela Rosario Salazar Ibáñez, Universidad La Salle, México

- Dr. Márcio Roberto de Lima, Universidade Federal de São João Del-Rei, Brasil

- Dra. Maria Alfredo Lopes Moreira, Universidade do Minho, Portugal

- Dra. María Ángeles Blanco Suárez, Universidad Complutense de Madrid, España

- Dra. María Bertha Fortoul Ollivier, Universidad La Salle, México

- Dra. Maria de Fátima Pereira, Universidade do Porto, Portugal

- Dra. María del Mar Molero Jurado, Universidad de Almeria, España

- Dra. María del Mar Rodríguez Rosell, Universidad Católica de Murcia, España

- Dra. María del Valle De Moya Martínez, UCLM, España

- Dra. María Domingo Coscollola, Universitat Internacional de Catalunya, España

- Dra. María Elena Ortiz, Universidad Politécnica Salesiana, Ecuador

- Dra. María Inés Copello Danzi, Universidad de la República, Uruguay

- Dra. Maria Inês Millen, Centro de Ensino Superior de Juiz de Fora, Brasil

- Dr. Maria Isabel Punín Larrea, Università Pontificia Salesiana, Italia

- Dra. María Jesús Martínez Usarralde, Universidad de Valencia, España

- Dra. María José Batista Pinto, Universidade Federal de Minas Gerais, Brasil

- Dra. María José Labrador-Piquer, Universitat Politècnica de València, España

- Dra. María José Serván, Universidad de Málaga, España

- María Margarita Marhx Bracho, Universidad La Salle, México

- Dra. María Sol Villagómez, Universidad Politécnica Salesiana, Ecuador

- Dra. María Teresa Prieto Quezada, Universidad de Guadalajara, México

- Dra. Maria-Carmen Caldeiro-Pedreira, Universidad Pública de Navarra, España

- Dra. Mariana Coralina Carmo, Universidade Estadual de Campinas, Brasil

- Dra. Maribel Enaida Alegre Jara, Universidad Nacional del Santa, Perú

- Dra. Maribel Pinto, Instituto Politécnico de Viseu, Portugal

- Dr. Mário Marcelo Coelho, Pontifícia Universidade Católica de São Paulo, Brasil

- Dr. Mario Rueda Beltrán, Universidad Nacional Autónoma de México, México

- Dr. Marlécio Maknamara, Universidade Federal da Bahia, Brasil

- Dra. Meireluci Costa Ribeiro, Universidade Federal de São Paulo, Brasil

- Dra. Milagros Elena Rodríguez, Universidad de Oriente, Venezuela

- Dra. Mirtes Lira, Universidade de Pernambuco, Brasil

- Dra. Mistli López Pérez, Universidad Autónoma del Estado de Morelos, México

- Dra. Mónica Cecilia Sáenz Amaguaya, Universidad Politécnica Salesiana, Ecuador

- Dra. Mónica Montenegro, Universidad de Buenos Aires, Argentina

- Dr. Nelson Rubén Martínez Reyes, Universidad Don Bosco, El Salvador

- Dra. Noelia Alcaraz Salarirche, Universidad de Málaga, España

- Dra. Noelia Ceballos Lopez, Universidad de Cantabria, España 
- Dra. Olga Alegre de la Rosa, Universidad de La Laguna, España

- Dra. Olga Espinoza Aros, Pontificia Universidad Católica de Chile, Chile

- Dr. Oscar Andrés Vivas Albán, Universidad del Cauca, Colombia

- Dr. Pablo Gómez Jiménez, Universidad Juárez Autónoma de Tabasco, México

- Dra. Paola Perochena González, Universidad Internacional de La Rioja, España

- Dra. Patricia Hora Correia, Universidade do Estado da Bahia, Brasil

- Dra. Patricia Muñoz Borja, Universidad Santiago de Cali, Colombia

- Dra. Patricia Torrijos, Universidad de Salamanca, España

- Dr. Paulo César Nodari, Universidade de Caxias do Sul, Brasil

- Dr. Pierre André Garcia Pires, Universidade Federal do Acre, Brasil

- Dr. Raimundo França, Universidade Federal de Alagoas, Brasil

- Dr. Ramon Luis de Santana Alcântara, Universidade Federal do Maranhão, Brasil

- Dr. Raúl Baños Navarro, Universidad de Almería, España

- Dra. Regina Ferreira Barra, Universidade Federal de Juiz de Fora, Brasil

- Dra. Renata Nunes Vasconcelos, Universidade Federal de Alfenas, Brasil

- Dra. Rita Gomes do Nascimento, Ministério da Educação, Brasil

- Dr. Robério Barreto, Universidade do Estado da Bahia, Brasil

- Roberto López Chila, Universidad Politécnica Salesiana, Ecuador

- Dra. Romilda Teodora Ens, Pontifícia Universidade Católica do Paraná, Brasil

- Dra. Ruth Aguilar Feijoo, Universidad Técnica Particular de Loja, Ecuador

- Dra. Ruth Amanda Cortés Salcedo, IDEP, Colombia

- Dra. Salomé Sola-Morales, Universidad de Santiago de Chile, Chile

- Dra. Sandra Alves da Silva Santiago, Universidade Federal da Paraíba, Brasil

- Dra. Sandra Liliana Londoño Calero, Pontificia Universidad Javeriana, Colombia

- Santos Daniel Castillo Gaona, Universidad Politécnica Salesiana, Ecuador

- Dr. Sebastián Granda Merchán, Universidad Politécnica Salesiana, Ecuador

- Dr. Sergio Enrique Hernández Loeza, UNAM, México

- Dra. Shirlei Rezende Sales, Universidade Federal de Minas Gerais, Brasil

- Dra. Shirley Silva, Universidade de São Paulo, Brasil

- Dra. Sibel Celik, Dicle University, Turquía

- Dra. Silvia Barco, Universidad Nacional del Comahue, Argentina

- Dra. Sílvia Ester Orrú, Universidade de Brasília, Brasil

- Dra. Sonia Koehler, Centro Universitário Salesiano de São Paulo, Brasil

- Dra. Sonia Liliana Ivanoff, UNPSJB, Argentina

- Dra. Sonia Sampaio, Universidad Federal de Bahía, Brasil

- Susana Barco, Universidad Nacional del Comahue, Argentina

- Dra. Suyai Malen García Gualda, Universidad Nacional del Comahue, Argentina

- Dra. Suzana dos Santos Gomes, Universidade Federal de Minas Gerais, Brasil

- Dra. Tânia Regina Dantas, Universidade do Estado da Bahia, Brasil

- Dra. Tania Suely Azevedo Brasileiro, Universidade Federal do Oeste do Pará, Brasil

- Dra. Teresa Laura Artieda, Universidad Nacional del Nordeste, Argentina

- Dr. Tiago Duque, Universidade Federal de Mato Grosso do Sul, Brasil

- Dr. Tomás Izquierdo Rus, Universidad de Murcia, España

- Dra. Váldina Gonçalves da Costa, UFTM, Brasil
- Dra. Vanessa Figueiredo, Universidade Federal de Mato Grosso do Sul, Brasil

- Dra. Vania Tanira Biavatti, Universidade Regional de Blumenau, Brasil

- Dr. Vicente Willians do Nascimento Nunes, Universidade Estácio de Sá, Brasil

- Dra. Victoria Jara Cobos, Universidad Politécnica Salesiana, Ecuador

- Dra. Victoria Vázquez Verdera, Universidad de Valencia, España

- Dra. Wedja Maria Oliveira Leal, PUC-SP, Brasil

- Dr. Wellington de Oliveira, UFVJM, Brasil

- Dr. Welson Barbosa Santos, Universidade Federal de Goiás, Brasil

- Willy Chambi Zabaleta, Universidad Salesiana de Bolivia, Bolivia

- Dra. Ximena Vélez Calvo, Universidad del Azuay, Ecuador

- Dra. Yadira Navarro Rangel, BUAP, México

- Dra. Yamila Irupé Núñez, Universidad Nacional de Misiones, Argentina

- Dra. Yamirlis Gallar Pérez, Universidad Internacional SEK, Ecuador

- Dra. Yolanda Castro Robles, Pontificia Universidad Javeriana, Colombia

\section{Consejo Técnico (Board of Management)}

- Dr. Ángel Torres-Toukoumidis

- Soledad Aguilar

- Wilson Verdugo (Soporte OJS)

- Marco Gutiérrez (Soporte OJS)

- Paulina Torres (Redacción y estilo)

- Martha Vinueza (Maquetación)

- Adriana Curiel (Traducción)

- Jessica Rivadeneira-Peñafiel (Asistente editorial)

\section{Edita (Published by)}

\section{Universidad Politécnica Salesiana}

www.ups.edu.ec

Consejo de Publicaciones (Board of Publications)

- Dr. Juan Cárdenas Tapia, Rector - Presidente

- Dr. Juan Pablo Salgado, Vicerrector de Investigación

- Dr. Luis Álvarez Rodas, Editor General

- Dr. Ángel Torres-Toukoumidis, Editor de Universitas

- Dr. Jaime Padilla Verdugo, Editor de Alteridad

- MSc. Sheila Serrano Vincenti, Editora de La Granja

- MSc. Jorge Cueva Estrada, Editor de Retos

- Dr. John Calle Sigüencia, Editor de Ingenius

- Dra. Floralba Aguilar Gordón, Editora de Sophia

- MSc. Betty Rodas Soto, Editora de Utopía

- Dr. Jorge Altamirano Sánchez, Editor Revista Virtual-Cátedra Unesco

- MSc. Mónica Ruiz Vásquez, Editora del Noti-Ups

- MSc. David Armendáriz González, Editor Web

- Dr. José Juncosa Blasco, Editorial Universitaria Abya-Yala

\section{Editorial Universitaria Abya-Yala}

(Quito, Ecuador)

Hernán Hermosa (Coordinación General)

Av. 12 de Octubre N23-116 y Wilson, Bloque A, UPS

Casilla 17-12-7-19

Teléfono: (593-2)3962-800 ext. 2638

E-mail: editorial@ayayala.org.ec

Portal electrónico: http://www.abyayala.org 


\section{ALTERIDAD}

p-ISSN: 1390-325x / e-ISSN: 1390-8642

Vol. 17, No. 1/ enero-junio 2022

\section{Índice}

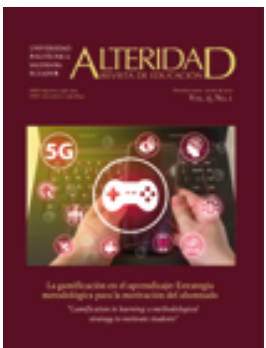

\section{La gamificación en el aprendizaje: Estrategia metodológica} para la motivación del alumnado

"Gamification in learning: a methodological strategy to motivate students"

\section{Editores Temáticos}

Dra. Carmen Llorente-Cejudo, Universidad de Sevilla (España)

Dr. Javier Gil-Quintana, Universidad Nacional de Educación a Distancia (España)

Dra. Alejandra Hurtado Mazeyra, Universidad Nacional de San Agustín (Perú)

Editorial

SECCIÓN MONOGRÁFICA (MONOGRAPHIC SECTION)

DR. DIEGO DE SOUSA MENDES, DR. MARCIO ROBERTO DE LIMA Y TAMARA APARECIDA REIS DE FREITAS

Gamificación, "No tengo ni idea de lo que es": un estudio en la Formación Inicial del Profesorado de Educación Física

Gamification, "I have no idea what it is": a study in the Physical Education Initial Teacher Training

DR. WILLIAM REYES-CABRERA

Gamificación y aprendizaje colaborativo en línea: un análisis de estrategias en una universidad mexicana

Gamification and collaborative online learning: an analysis of strategies in a Mexican university

LORENA MARTÍN-PÁRRAGA, ANTONIO PALACIOS-RODRÍGUEZ Y DR. ÓSCAR MANUEL GALLEGO-PÉREZ

Jugamos o gamificamos? Evaluación de una experiencia formativa sobre gamificación para la mejora de las competencias digitales del profesorado universitario

Do we play or gamify? Evaluation of gamification training experience to improve the digital competence of university teaching staff

VIVIANA MALVASI Y DAVID RECIO-MORENO

Percepción de las estrategias de gamificación en las escuelas secundarias italianas

Perception of gamification strategies in Italian secondary school

CARMEN NAVARRO-MATEOS Y DR. ISAAC J. PÉREZ-LÓPEZ

Una app móvil potencia la motivación del alumnado en una experiencia de gamificación universitaria

A phone app as an enhancer of students' motivation in a gamification experience in a university context

\section{SECCIÓN MISCELÁNEA (MISCELLANEOUS SECTION)}

DR. FERNANDO GONZÁLEZ-ALONSO, DRA. AZUCENA OCHOA-CERVANTES Y DR. JOSÉ LUIS GUZÓN-NESTAR.......... Aprendizaje Servicio en Educación Superior entre España y México. Hacia los ODS

Service-learning in higher education between Spain and Mexico. Towards the SDGS

DRA. MARÍA VERÓNICA LEIVA-GUERRERO, JIMENA IVONNE SANHUEZA-MANSILLA, MARÍA PAZ SOTO-CALDERÓN Y MARÍA ELIANA MUÑOZ-LAMELES

Monitoreo de aprendizajes en escuelas públicas chilenas en contexto COVID-19

Learning monitoring at low income schools in COVID-19 context

RITA DE CÁSSIA DE ALMEIDA REZENDE Y DR. GERALDO CALIMAN.

Interacción, diálogo y prácticas pedagógicas en el bachillerato

Interaction, dialogue and pedagogical practices in high school

JUSTO OLIVARES-ROSADO, DRA. ISABEL LÓPEZ-COBO Y DR. JESÚS CONDE-JIMÉNEZ

Estudio motivacional sobre el aprendizaje de la religión en Educación Básica mediante las TIC

Motivational study on learning religion in Basic Education using ICT

DR. RODOLFO ANTONIO PADILLA-BERDUGO, JORGE ALBERTO AMADOR-LÓPEZY YR. JOSÉ LUIS OLIVO-FRANCO Fijaciones visuales y caracteres: incidencias en la comprensión de textos expositivos

Visual fixations and characters: incidence in the understanding of expository texts

Normas de Publicación en "Alteridad" 


\section{ALTERIDAD}

p-ISSN: 1390-325x / e-ISSN: 1390-8642

Vol. 17, No. 1/ enero-junio 2022

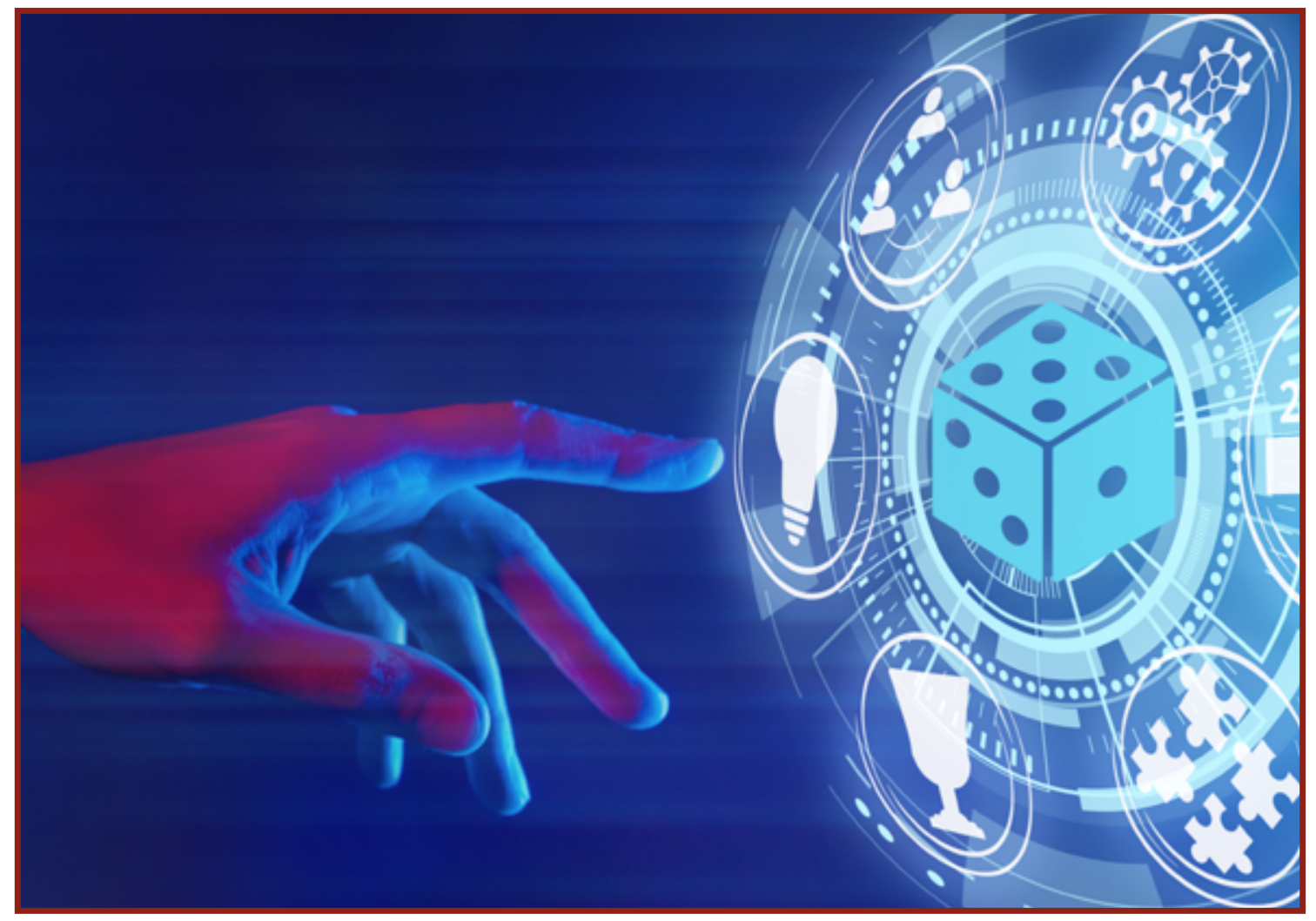

Fuente: https:// www.shutterstock.com/es/image-photo/gamification-gaming-technology-illustration-neon-style-1917566459

\section{Gamificación}

La gamificación permite usar elementos de la dinámica de los juegos en la realidad educativa para conseguir los objetivos de aprendizaje planteados a través del uso de distintas técnicas y recursos. De ese modo, se otorga un abordaje lúdico y activo a los procesos de enseñanza-aprendizaje donde los y las estudiantes tienen un rol protagónico y participativo mientras obtienen una experiencia de aprendizaje divertida, motivadora y positiva. 


\section{ALTERIDAD \\ P-ISSN: 1390-325x / e-ISSN: 1390-8642 \\ Vol. 17, No. 1/ enero-junio 2022}

\section{Editorial}

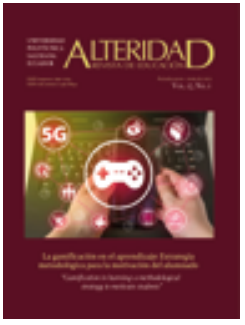

Editores Temáticos

- Dra. Carmen Llorente-Cejudo, Universidad de Sevilla (España)

- Dr. Javier Gil-Quintana, Universidad Nacional de Educación a Distancia (España)

- Dra. Alejandra Hurtado-Mazeyra, Universidad Nacional de San Agustín (Perú)

EDITOR JEFE

- Dr. Jaime Padilla-Verdugo, Universidad Politécnica Salesiana (Ecuador)

La gamificación se incorpora en el ámbito educativo como una tendencia pedagógica incrementada exponencialmente tanto por el uso de los dispositivos digitales, como por la incorporación de los juegos en formato portátiles entre estudiantes, propiciando un aprendizaje ubicuo. Unas características del aprendizaje acrecentadas por y en la sociedad posdigital y sus aplicaciones hacen que, a través de la gamificación, se sugiera el uso de elementos de juego que se descubren como una espiral de una actividad, de una práctica, o de una propuesta formativa que posee una clara intencionalidad final. Supone establecer un objetivo concreto, que es al que habrá que focalizar la atención en los entornos de aprendizaje analógicos y digitales para poder hacer un correcto uso de la gamificación.

En el contexto concreto del mundo educativo, son muchas las prácticas en las que la gamificación ha obtenido ya un gran éxito, bien debido a que ha alcanzado un grado alto de aceptación en su sistema de motivación extrínseca (donde las recompensas hacen muy bien su labor de alentar el camino), o porque el entorno simulado que se proporciona genera una sensación de seguridad al alumnado.

Una metodología donde lo tecnológico y lo digital adquieren un papel primordial al llevar a cabo dichas prácticas, pero que curiosamente se presenta como una estrategia que no necesita inexorablemente de ellos, ya que puede desarrollarse desde un nivel analógico sin ningún tipo de dificultad. Todos estos aspectos, otorgan a la gamificación un amplio abanico de posibilidades que, a través de sus temáticas prioritarias (gamificación en educación, resultados desde experiencias gamificadas, gamificación y narrativa transmedia, entre otros), serán el reflejo de avances en procesos de aprendizajes gamificados, planes formativos, experiencias, diseño y resultados de metodologías gamificadas, e investigaciones en las que esta estrategia sea su objeto de estudio central.

Desde este número monográfico de la «Revista Alteridad», el equipo de editores ha querido profundizar e indagar en las reflexiones, estudios y conclusiones con el propósito de generar un marco desde el que analizar las experiencias de innovación educativa en los escenarios educativos; desde la profundización en los conceptos, definiciones, características y clasificación de las acciones formativas gamificadas, hasta qué implicaciones se pueden extrapolar en la formación digital del docente o del alumnado.

Con el entusiasmo de contribuir a la formación de educadores e investigadores interesados por esta metodología, la Sección Monográfica "La gamificación en el aprendizaje: Estrategia metodológica para la motivación del alumnado" se articula en torno a cinco manuscritos. Bajo el título "Gamificación, "No tengo ni idea de lo que es": un estudio en la Formación Inicial del Profesorado de Educación Física", los autores De Sousa, De Lima y Reis, a través de un estudio cualitativo, tratan de identificar aquellos elementos más relevantes en profesores de formación inicial (PEFI), desta- 
cando entre sus resultados más significativos que la asociación de las acciones gamificadas con las tecnologías digitales potencia el desarrollo de la educación en la cultura digital. La sección continúa con un análisis cuantitativo de una experiencia de gamificación mediante tres estrategias diferentes: Triada PET, Story Telling y Escape Room. Del artículo de William Reyes, titulado "Gamificación y aprendizaje colaborativo en línea: un análisis de estrategias en una universidad mexicana" se destaca que las tres estrategias utilizadas incidieron de manera significativa en el aprendizaje colaborativo, y que estas son determinantes para lograr el aprendizaje colaborativo en línea. Por otro lado, y desde la formación del profesorado universitario, la gamificación se trabaja desde la universidad para conseguir y desarrollar habilidades óptimas que beneficien al alumnado, tanto personal como profesionalmente, donde desde una universidad de América Latina se estudió la percepción de la estrategia metodológica a través del modelo de Aceptación de la Tecnología (TAM). Tanto Martín como Palacios y Gallego, exponen bajo el título “¿Jugamos o gamificamos? Evaluación de una experiencia formativa sobre gamificación para la mejora de las competencias digitales del profesorado universitario" cómo todos los participantes percibieron esta estrategia adecuada, resaltando dimensiones como: facilidad de uso en el aula, integración, transformación del interés en el aprendizaje o capacidad para mostrar una actitud positiva en su uso.

Si existe un área que presenta grandes dificultades a la hora de asimilar conceptos, probablemente sea el de las matemáticas. Así, desde un estudio multicaso denominado "Percepción de las estrategias de gamificación en las escuelas secundarias italianas" combinando técnicas cuantitativas y cualitativas, y con una muestra de 4845 estudiantes, más 12 entrevistas al profesorado, ofrecen resultados muy interesantes como que el alumnado apenas percibe el uso de juegos, analógicos o digitales, por el profesorado, y al mismo tiempo, manifiestan tener escaso conocimiento sobre la gamificación y sus posibilidades. Es por ello que Malvasi y Recio señalan en sus conclusiones la necesidad de una sólida formación del alumnado y profesores para optimizar las posibilidades de la gamificación en los escenarios educativos.

Por último, desde la incorporación de las aplicaciones educativas y el uso de dispositivos móviles a la estrategia de gamificación, se presenta el último estudio que, bajo el título "Una app móvil potencia la motivación del alumnado en una experiencia de gamificación universitaria" describe la aplicación «\$in TIME» diseñada ad hoc con la intención de gestionar los diferentes elementos que conformaron el proyecto de gamificación basado en la película «In Time» en espacios universitarios.

Esperamos, al igual que les sucede a los editores temáticos que han tenido el privilegio de formar parte de este monográfico, que este se convierta en un punto de referencia para investigadores y docentes que estén interesados en identificar buenas prácticas educativas vinculadas con la estrategia de la gamificación, permitiéndole avanzar en la obtención de resultados y conclusiones futuras que repercutan de manera directa o indirecta en el ejercicio de su práctica docente e investigadora.

La Sección Miscelánea incluye temáticas diversas relacionadas con la educación, en particular, con el aprendizaje como el aprendizaje-servicio, el aprendizaje en contexto del COVID-19, la interacción social entre profesores y alumnado, la motivación en el aprendizaje mediante el uso de las TIC y la incidencia de las fijaciones visuales en la comprensión de textos. El artículo "Aprendizaje Servicio en Educación Superior entre España y México. Hacia los ODS”, trata una temática actual e interesante dando cuenta, por una parte, de los fundamentos filosóficos y pedagógicos y de los modelos que lo sustentan; y por otra, de las experiencias diseñadas desde algunas universidades españolas y mexicanas, aplicadas por estudiantes en sectores sociales vulnerables vinculados a la niñez, la adolescencia, la juventud, la mujer, las comunidades indígenas, entre otros. El resultado de esas experiencias de aprendizaje en contextos reales, no solo generan motivación y crecimiento personal en estudiantes, 
sino también su compromiso social, orientados al logro progresivo de los ODS. El artículo visibiliza las posibilidades que la ApS ofrece para articular teoría y práctica en la formación universitaria, así como para fortalecer y dar más coherencia al trabajo que las universidades realizan en el ámbito de la vinculación con la sociedad.

En el contexto de la pandemia producto de la COVID-19, conocer cómo evaluar el aprendizaje se tornó muy relevante. El artículo "Monitoreo de aprendizajes en escuelas públicas chilenas en contexto COVID-19" trata de responder a esa problemática que algunos investigadores denominan proceso de enseñanza-aprendizaje de emergencia. Esta investigación brinda la oportunidad de pensar sobre las condiciones de aprendizaje y evaluación en clases no presenciales. Las entrevistas en profundidad realizadas a directivos muestran la diversidad de concepciones y propósitos del monitoreo, su sistematicidad y la concentración de la comunicación en las redes sociales como WhatsApp y correo electrónico, así como también las dificultades para atender a la diversidad y singularidad de cada estudiante.

Con el propósito de analizar cómo la escuela y, en particular, el profesorado contribuye a la formación autónoma y dinámica del estudiantado, el artículo "Interacción, diálogo y prácticas en el bachillerato" propone el estudio de caso de una escuela pública de Ceilândia (Brasil), con un alto nivel de vulnerabilidad por cuestiones socioeconómicas, violencia, problemas familiares, embarazos adolescentes y drogadicción. La triangulación entre el análisis documental, las entrevistas semiestructuradas y la observación participante, permite identificar diversos círculos de exclusión; y proponer estrategias pedagógicas basadas en metodologías activas, en la que los y las estudiantes de manera resiliente, se convierten en protagonistas de su propio aprendizaje.

A partir de la concepción de que la motivación constituye el motor para el aprendizaje, el artículo "Estudio motivacional sobre el aprendizaje de la religión en Educación Básica mediante las TIC" busca identificar las causas de la desmotivación en el aprendizaje de la religión en una institución de Educación Básica. Los resultados del estudio realizado con 268 estudiantes y ocho profesores de religión, evidencian bajos niveles motivacionales debido a deficiencias en los ambientes de aprendizaje y al uso de metodologías de enseñanza tradicionales; para incrementar la motivación se sugiere el uso de metodologías participativas mediadas por las TIC.

El artículo "Fijaciones visuales y caracteres: incidencias en la comprensión de textos expositivos" completa la sección miscelánea. La comprensión lectora tiene varios factores sociales, culturales y emocionales que puede incrementar o disminuir sus niveles e incidir en la motivación para el aprendizaje; entre ellos se analiza de manera original en este estudio, la fijación visual. La investigación se realiza con la participación de 80 estudiantes de secundaria, quienes leen tres textos relacionados con el electromagnetismo en la pantalla Eye tracher T.120, la cantidad de fijaciones se analiza mediante el software Tobii 3.4.6. Los resultados indican que las fijaciones visuales cumplen un rol importante en la comprensión lectora puesto que la información ingresa a través de la vista.

Este número de Alteridad se cierra con la siguiente interrogante ¿Se puede hablar de una educación pospandemia? Han transcurrido dos años desde el inicio del COVID-19 y todavía no existe una respuesta científica unificada acerca del comportamiento del virus y de las personas; y, en consecuencia, cualquier planificación se torna emergente y coyuntural. Si bien, por una parte, se ha incrementado considerablemente el uso de las TIC para el proceso de enseñanza-aprendizaje de manera innovadora; por otra, la pandemia ha dejado al descubierto que no todas las familias poseen celulares, tablet o computadores, y menos internet. En este contexto, se plantean diversos desafíos para la educación: ¿cómo y cuándo ayudar al estudiantado que abandonaron la escuela a reincorporarse a sus estudios? ¿la modalidad híbrida llegó para quedarse? ¿Las diversas Apps son válidas y confiables?; qué decir de la flexibilidad curricular, de la validez de la evaluación en línea, de la calidad 
académica con la que egresan los nuevos profesionales. Existen más preguntas que respuestas, que se espera podamos ir resolviendo juntos maestros, investigadores, estudiantes, informáticos, psicólogos, sociólogos, políticos, entre otros. Esperamos que Alteridad se convierta en ese espacio de discusión para debatir los nuevos retos.

Se invita a profesores e investigadores a enviar artículos para el próximo monográfico sobre "Enseñanza y aprendizaje de las matemáticas: diferentes enfoques y niveles educativos". La Convocatoria estará abierta hasta el 28 de febrero del 2022. 


\section{Sección Monográfica}

(Monographic section)

\section{La gamificación en el aprendizaje: Estrategia metodológica para la motivación del alumnado}

\section{"Gamification in learning: a methodological strategy to motivate students"}

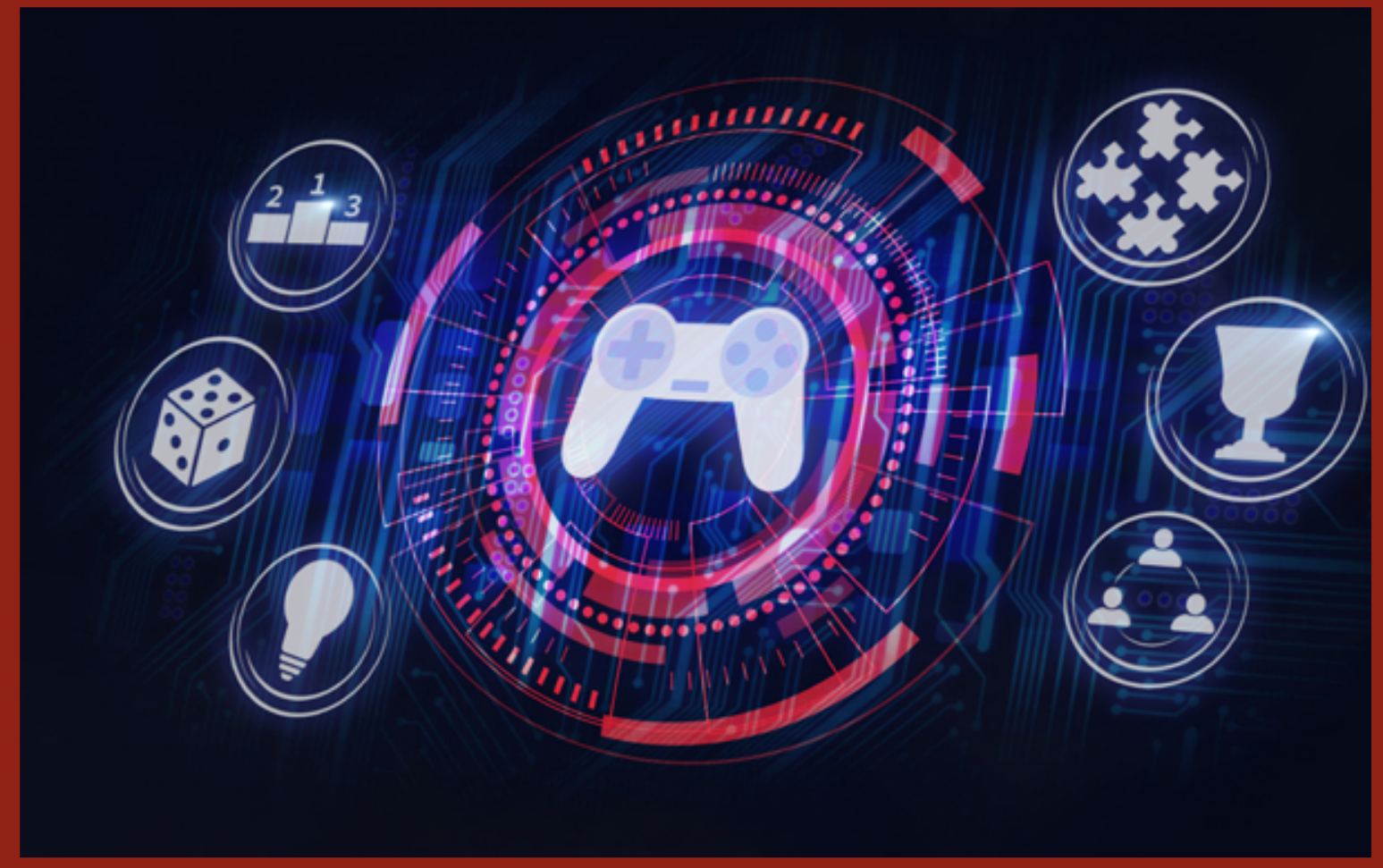

Fuente: https:/ / www.shutterstock.com/es/image-illustration/gamification-business-processes-game-development-concept-1869188803 


\section{EAाERाDa Gamificación, "No tengo ni idea de lo que es": un 56 \% 91 estudio en la Formación Inicial del Profesorado de Educación Física}

\section{Gamification, "I have no idea what it is": a study in the Physical Education Initial Teacher Training}

Dr. Diego de Sousa Mendes es profesor e investigador de la Universidad Federal de São João del-Rei (Brasil) (diegomendes@ufsj.edu.br) (https://orcid.org/0000-0002-2297-5883)

(D) Dr. Marcio Roberto de Lima es profesor de la Universidad Federal de São João del-Rei (Brasill) (marcinholima@ufsj.edu.br) (http://orcid.org/0000-0003-3790-1 104)

(1) Tamara Aparecida Reis de Freitas es profesora en la Universidad Federal de São João del-Rei (Brasil) (tamarinhafreitas22@ gmail.com) (https://orcid.org/0000-0001-9774-8696)

Recibido: 2021-08-31 / Revisado: 2021-11-05 / Aceptado: 2021-11-26 / Publicado: 2022-01-01

\section{Resumen}

La composición de estrategias didácticas que impliquen la imbricación de elementos y lenguajes propios de la cultura digital al contexto de la formación educativa es una condición indispensable para la renovación de la acción pedagógica en la contemporaneidad. En este sentido, esta investigación tuvo como objetivo identificar elementos relevantes en un proceso de enseñanza desarrollado con 24 profesores en formación inicial (PEFI) en relación con la gamificación. De naturaleza cualitativa, este estudio consolidó un Estudio de Caso estructurado a partir de las experiencias pedagógicas desarrolladas en el componente curricular "Metodología de la Enseñanza de la Educación Física III". Los datos empíricos se produjeron a partir de observaciones de las clases con registros en un diario de campo y grabaciones digitales de audio de los debates, que fueron transcritas. Este corpus fue sometido a un Análisis de Contenido mediado por el software ATLAS.ti. Los resultados indican que el proceso de aprendizaje de la gamificación se desarrolló a partir de la experiencia de crear clases gamificadas. La presencia, el énfasis o la ausencia de ciertos elementos de juego fueron indicadores de la comprensión que el grupo de PEFI estableció respecto al tema. También cabe destacar que la asociación de las acciones gamificadas con las tecnologías digitales ha potenciado el desarrollo de la educación en la cultura digital. Finalmente, la experiencia se realiza forjando posibilidades de significación y aprendizaje al asumir la comunicación y la reflexión como elementos mediadores de las acciones pedagógicas gamificadas.

Descriptores: Gamificación, formación, docente, enseñanza, educación.

\begin{abstract}
The implementation of didactic strategies that involve the imbrications of elements and specific languages of digital culture in the context of teacher training is an indispensable condition for the renewal of contemporary pedagogical action. This research aimed to identify relevant elements in a teaching process developed with 24 teachers in initial training (TIT) regarding gamification. This study consolidated a Case Study structured from the pedagogical experiences developed during the "Physical Education Teaching Methodology III." The empirical data were produced through classroom observations with records in a field diary and digital audio recordings of debates held, which were transcribed. This corpus was submitted to a Content Analysis mediated by the ATLAS. ti software. The results indicated that the learning process about gamification developed from the experience of creating gamified classes. The presence, emphasis, or absence of certain game elements revealed the understanding that the TIT group established concerning thematic. It is also noteworthy that the association of gamified actions to digital technologies boosted education development in digital culture. Finally, the experience is undertaken forged possibilities of meaning and learning by assuming communication and reflection as mediating elements of gamified pedagogical actions.
\end{abstract} education.

Keywords: Gamification, teacher, training, teaching,

Forma sugerida de citar: Mendes, D., Lima M., \& Freitas, T. (2022). Gamificación, "No tengo ni idea de lo que es": un estudio en la Formación Inicial del Profesorado de Educación Física. Alteridad, (17)1, 12-23. https://doi.org/10.17163/alt. v17n1.2022.01 


\section{Introducción}

Las formas de sociabilidad contemporánea se han ido reconfigurando con la mediación de las Tecnologías Digitales de la Información y la Comunicación (TDIC). Nuevos formatos de acceso, uso/tratamiento y producción de información se diversifican con estas tecnologías, ampliando la comunicación móvil, la computación ubicua y la (inter)acción social en los ciberespacios.

Este escenario socio-técnico tipifica la cultura digital (Lemos \& Lévy, 2010; De Barros, 2019; Lemos, 2020), que, a partir del uso intensivo de las tecnologías digitales en red, interpone cambios expresivos en todos los sectores de las actividades humanas. Entendiendo que toda esta gama de transformaciones no está determinada por los artefactos técnicos digitales, sino condicionada por/involucrada con ellos, admitimos la existencia de muchos desafíos que involucran aspectos éticos, socioeconómicos, de inclusión digital, de seguridad de la información personal, de predicción del comportamiento, etc.

En este campo, entendemos la urgencia de la composición de estrategias que impliquen la imbricación de elementos y lenguajes propios de la cultura digital al contexto de la formación educativa. Con el propósito que estos espacios se muestren como incluyentes y promotores de la inclusión en esta cultura. Es una posición que acoge el pensamiento de que la educación también se ve impulsada a reconfiguraciones y necesita ser compatible con la cultura digital, siendo indispensable para comprender estas transformaciones y desarrollar otras formas de educar (Lima, 2013).

Con este enfoque, dirigimos nuestra atención a la formación docente inicial, buscando acercarla a una estrategia derivada del lenguaje y diseño de los juegos, denominada gamificación. Inicialmente, señalamos que "[...] la gamificación consiste en el uso de elementos típicos de los juegos (desafíos, colaboración, resolución de problemas, retroalimentación continua, etc.) en contextos distintos a los juegos" (Carvalho \&
Lima, 2019, p. 1204). En este particular, reconocemos los juegos electrónicos como productos culturales expresivos de la cultura digital, y que pueden contribuir a la creación de "[...] espacios de aprendizaje mediados por el desafío, el placer y el entretenimiento" (Alves et al., 2014, p. 76).

Con esto en mente, se propuso a un grupo de profesores en formación inicial (PEFI) una experiencia pedagógica para acercarlos a la gamificación. Dicha experiencia ocurrió en 2019, a partir de un componente curricular del curso de Pregrado en Educación Física de la Universidad Federal de São João del-Rei (UFSJ). Se propuso a los PEFI la producción y el análisis colectivo de una unidad didáctica centrada en los alumnos y las alumnas de secundaria, que fueron subvencionados por los fundamentos de la gamificación como estrategia de enseñanza. Nuestro problema de investigación fue indagar: ¿qué elementos eran relevantes en un proceso de enseñanza desarrollado con profesores en formación inicial en relación con la gamificación?

\subsection{Gamificación y formación del profesorado}

El término gamificación fue utilizado por primera vez en 2003 por el programador informático e inventor británico Nick Pelling, con la semántica de aplicar conceptos de diseño de juegos para "[...] hacer que las transacciones electrónicas sean rápidas y agradables" (Nanodome, 2011). Sin embargo, recién en 2011, el concepto comienza a destacarse en el sentido de agregar valor a categorías de negocios y aprendizajes diversos (Alves, 2015).

Aunque existen diferentes conceptualizaciones para la gamificación (Apostol et al., 2013; Kim, 2011; Deterding et al., 2011), asumimos su concepto como el "uso de la mecánica, la estética y el concepto de los juegos, con el objetivo de proporcionar compromiso entre las personas, motivar acciones, fomentar el aprendizaje y promover la resolución de problemas" en escenarios no lúdicos (Kapp, 2012, p. 336, traducción propia). 
Para ello, se incorporan sistemáticamente elementos presentes en los juegos a situaciones no lúdicas. Existen muchas definiciones de elementos de juego que se explicitan en la caracterización del proceso de gamificación (McGonigal, 2011; Zichermann \& Cunningham, 2011; Werbach \& Hunter, 2012; Kapp, 2012). Entre ellas se encuentran las metas, los objetivos, los personajes, las reglas, los sistemas de retroalimentación, los niveles y etapas, los logros, las insignias, etc. En este estudio, consideramos la definición de elementos de juego de Werbach y Hunter (2012), quienes los organizan en tres tipos generales: dinámicas, mecánicas y componentes. Las dinámicas se refieren a los elementos más generales y abstractos, que no forman parte directamente del juego, pero crean el ambiente. Las mecánicas son objetivas y guían las acciones de los jugadores en la dirección deseada, delimitando lo que el jugador puede o no hacer dentro del juego. Y los componentes son aplicaciones específicas visualizadas y utilizadas en la interfaz del juego.

La gamificación en la educación implica el desarrollo de estrategias didácticas que promueven transformaciones en la esfera del profesor y del alumno. Para el profesorado, la gamificación interfiere en la forma de organizar los contenidos y las dinámicas de enseñanza y se planifica de manera que se fomente la incorporación de recursos de aprendizaje interactivos y estimulantes, lo que puede implicar la valorización de las tecnologías y los lenguajes de la cultura digital. Al diseñar espacios de aprendizaje más evocadores para el alumnado, se espera que se comprometan en la resolución de problemas, dando sentido a lo que hacen y aprenden.

En particular, una acción gamificada extrapola la postura de pasividad/receptividad, exigiendo a los participantes que se movilicen en la consecución de los objetivos de aprendizaje. En este sentido, los aspectos colaborativos y cooperativos de los juegos pueden integrar la planificación gamificada y favorecer la formación de la inteligencia colectiva (Lévy, 2007). Como explican Alves et al. (2014, p. 81) en "[...] las acciones gamificadas que requieren prácticas colaborati- vas, el concepto de inteligencia colectiva de Lévy se ve reforzado ya que los jugadores necesitan interactuar, intercambiar experiencias y conocimientos para realizar una tarea determinada”.

La gamificación en un contexto educativo no se basa en formatos prescriptivos y/o condicionantes, recayendo en la sobrevaloración de un sistema de recompensas frente a los estímulos. Por el contrario, defendemos que las estrategias gamificadas pueden fomentar procesos reflexivos que permitan a los participantes adoptar una postura crítica sobre lo que aprenden e incluso sobre la propia intervención que media en el proceso de enseñanza. Además, también debemos reconocer que:

Características como la distribución de las puntuaciones de las actividades, el suministro de retroalimentación y el fomento de la colaboración en los proyectos son los objetivos de muchos planes pedagógicos. La diferencia es que la gamificación proporciona una capa más explícita de interés y un método para coser estos elementos para lograr la similitud con los juegos. (Fardo, 2013 p. 63)

En esta dirección, según Alves et al. (2014), una actividad gamificada tendría que englobar en su planificación los siguientes aspectos: i) Que los profesores conozcan, experimenten y utilicen los juegos; ii) Adaptar las acciones a los participantes; iii) Definir el alcance de los contenidos referenciales, las habilidades a desarrollar, así como las actitudes a potenciar; iv) Comprender los problemas que pueden ser explorados como contenidos narrativos y/o gamificados; v) Delimitar el objetivo de la estrategia gamificada considerando su adhesión al alcance definido; vi) Construir una narrativa, considerando su compatibilidad con el tema y el contexto; vii) Definir la(s) plataforma(s) y los recursos (salas físicas/virtuales, mensajeros electrónicos, equipos, etc.) adecuados para el desarrollo del tema y las acciones contempladas; viii) Tareas de diseño y dinámicas para su consecución. 
Por lo tanto, reconocemos que los elementos típicos del diseño de juegos, cuando se incorporan a las dinámicas educativas, pueden contribuir a estructurar el trabajo del profesor y a mejorar el rendimiento de los alumnos y las alumnas, haciendo que el aprendizaje sea más eficaz. Observamos que la adopción de la gamificación en la educación no asegura, por sí misma, un cambio de comportamiento en lo que respecta al aprendizaje y al compromiso. Asimismo, admitimos que la gamificación no es una solución única y definitiva para los problemas educativos, que implican la infraestructura, la valoración de la carrera docente y la reconfiguración de los procesos de formación, entre otros aspectos.

\section{Metodología}

La investigación realizada fue cualitativa (Bogdan \& Biklen, 1994; Minayo, 2012), del tipo Estudio de Caso (Yin, 2015; André, 2005). Participaron en el estudio 24 profesores en formación inicial (9 mujeres identificadas y 15 hombres). Los sujetos colaboraron espontáneamente con la investigación, habiendo sido informados desde el principio sobre sus procedimientos y objetivos.

La experiencia se realizó en el componente curricular de Metodología de la Enseñanza de la Educación Física III e incluyó la elaboración de diez clases secuenciales, denominadas Interlocuciones Pedagógicas (IP). Antes del desarrollo de los seminarios prácticos, hubo una primera etapa del estudio -Etapa 1- que consistió en un período de
Preparación, en el que se desarrollaron colectivamente los estudios teóricos y un guion con el tema y el objetivo de cada uno de los 10 IP, permitiendo que el trabajo culminara en la planificación secuencial de diez (10) clases coherentemente articuladas. La etapa I duró un mes, con reuniones dos veces por semana de 50 minutos.

La Etapa 2 se ocupó de la Organización de los seminarios prácticos. En esta etapa, la IP se basó en la gamificación, dirigida a estudiantes de secundaria, y se presentó en planes de trabajo escritos. El contenido curricular elegido para el experimento fue el Parkour, ${ }^{1}$ y el PEFI organizó colectivamente diez planes de clase. El PEFI se organizó en cinco grupos de trabajo, cada uno de los cuales presentó un seminario. La segunda etapa tuvo la misma duración y organización temporal que la primera.

La tercera etapa se ocupó de la aplicación y el análisis de las IP. Las IP se presentaron y discutieron en cinco semanas consecutivas, con dos clases semanales de dos horas de duración. La dinámica establecida preveía que cada grupo actuara en tres roles a lo largo de los seminarios 1) Instructores: responsables por la implementación de dos IP secuenciales en un solo día; 2) Evaluadores: analizaban la IP realizada por el grupo de profesores de la semana; 3 ) Participantes: actuaban como alumnos/as de la escuela secundaria —alumnos/as de la IP implementada. El cuadro 1 presenta la forma en que se organizó el trabajo:

Tabla 1. Organización del trabajo de los grupos en la disciplina

\begin{tabular}{|l|l|l|l|l|l|}
\hline \multicolumn{1}{|c|}{$\begin{array}{c}\text { Semana } \\
\text { Grupo }\end{array}$} & \multicolumn{1}{|c|}{ Semana 1 } & \multicolumn{1}{|c|}{ Semana 2 } & \multicolumn{1}{|c|}{ Semana 3 } & \multicolumn{1}{c|}{ Semana 4 } & \multicolumn{1}{|c|}{ Semana 5 } \\
\hline Grupo 1 & Instructores & Participantes & Participantes & Participantes & Evaluadores \\
\hline Grupo 2 & Evaluadores & Instructores & Participantes & Participantes & Participantes \\
\hline Grupo 3 & Participantes & Evaluadores & Instructores & Participantes & Participantes \\
\hline Grupo 4 & Participantes & Participantes & Evaluadores & Instructores & Participantes \\
\hline Grupo 5 & Participantes & Participantes & Participantes & Evaluadores & Instructores \\
\hline
\end{tabular}


Las reuniones de disciplina se organizaron de manera que, en la primera clase de la semana, el grupo de Instructores desarrollara su IP, y en la segunda clase de la semana, se realizara un debate colectivo coordinado por el grupo de evaluadores.

Los datos fueron recolectados entre septiembre y noviembre de 2019, considerando las etapas de "Preparación, Organización e Implementación y Análisis de las IP" desarrolladas por el PEFI. La recolección de datos se realizó a través de observaciones de las clases, registros de diarios de campo y grabaciones digitales de audio de las discusiones sostenidas, que posteriormente fueron transcritas.

El análisis de datos se realizó mediante el Análisis de Contenido (AC) de Bardin (2016), con una organización Categórica Temática, con el apoyo del software ATLAS.ti, versión 7.5.7.

El análisis de contenido (AC) se organiza metodológicamente en tres etapas, según Bardin (2016): i) Pre-análisis; ii) Exploración del material y iii) Tratamiento de los resultados, inferencia e interpretación. En nuestro estudio, en la fase de pre-análisis se realizó una lectura cuidadosa de los diarios de campo y de las transcripciones de los debates para identificar los principales temas planteados. Cada sección de los diarios y de las transcripciones de los debates se organizó por fecha y se incorporó al software ATLAS.ti. El software aloja las fuentes primarias en un archivo denominado Hermeneutic Unit (Unidad Hermenéutica), y las secciones de este corpus se identificaron con una sigla cada una: de D1 a D6. Las transcripciones se identificaron por T1 a T6.

La fase de exploración del material permitió referencias mediante el registro de índices o, como en ATLAS.ti se denomina codes (códigos). Se trata de palabras clave que indican los núcleos de significado relevantes para el estudio y que se producen a partir del corpus, evitando una perspectiva a priori. El trabajo de indexación sumó 32 codes.

Posteriormente, se verificó el contexto en el que se utilizaba cada code, lo que en el AC se denomina unidades de contexto. En ATLAS.ti, las unidades de contexto se refieren como quotation (citas). Esto permitió verificar el contexto semántico en el que se utilizaron los codes.

A continuación, fue posible organizar los codes en grupos con un núcleo común de significado. Según el AC, en el software estos grupos se denominan Family (Familia) y constituyen las categorías empíricas del análisis. En total, se constituyeron tres categorías empíricas a posteriori que engloban los significados del PEFI:

\section{Tabla 2. Categorías empíricas}

\begin{tabular}{|l|l|l|}
\cline { 2 - 3 } & \multicolumn{1}{|c|}{ Título de la categoría } & \multicolumn{1}{c|}{ Contenido } \\
\hline Categoría 1 & $\begin{array}{l}\text { Aprendizaje de la comprensión de los elementos } \\
\text { de la gamificación. }\end{array}$ & $\begin{array}{l}\text { presenta la comprensión de los elementos de la } \\
\text { gamificación por parte de los PEFI }\end{array}$ \\
\hline Categoría 2 & $\begin{array}{l}\text { Interfaces entre la gamificación, las TDIC y la cul- } \\
\text { tura digital }\end{array}$ & $\begin{array}{l}\text { se refiere a la interfaz de la gamificación con la cul- } \\
\text { tura digital y la presencia de las tecnologías digita- } \\
\text { les en la acción }\end{array}$ \\
\hline Categoría 3 & Relación teórico-práctica en debate & $\begin{array}{l}\text { la tensión entre teoría y práctica a partir de un ejer- } \\
\text { cicio crítico y creativo }\end{array}$ \\
\hline
\end{tabular}




\section{Resultados y discusión}

Identificamos que los PEFI inicialmente no sabían lo que era la gamificación, expresando frases como: "no tengo ni idea de lo que es", "no lo sé" "creo que podría ser algo relacionado con el uso de videojuegos en clase. Pero, ¿cómo sería esto?" (D1, 28/09/2019). Su asociación con el uso de videojuegos en las clases escolares también fue evidente. Esto indica la relevancia de dar visibilidad a la comprensión de la gamificación en la formación inicial del profesorado, como ya lo señalan Martins y Giraffa (2015), explicitando sus preceptos teórico-metodológicos, así como su distinción del acto de jugar, crear juegos educativos, o incluso la inclusión de juegos electrónicos en la rutina y los procesos educativos.

A pesar de una comprensión inicialmente limitada de la gamificación, nuestros registros indican un avance en las acciones y manifesta- ciones de los investigados, que pasaron a entender el término ya no como sinónimo de "uso de juegos electrónicos" en el contexto de las acciones escolares, sino como una posibilidad de transposición creativa de elementos de diseño de juegos a diversas situaciones, especialmente para la organización de los dispositivos educativos escolares. Los elementos que fundamentan esa re-significación se constituyeron a partir de las categorías que siguen.

\subsection{Aprendizaje a partir de la com- prensión de los elementos de la gamificación}

Observamos una apropiación gradual por parte de los PEFI de ciertos elementos de juego que caracterizan la gamificación en la IP, como se muestra en el Gráfico 1.

\section{Gráfico 1. Frecuencia de los elementos de gamificación en las interlocuciones pedagógicas}

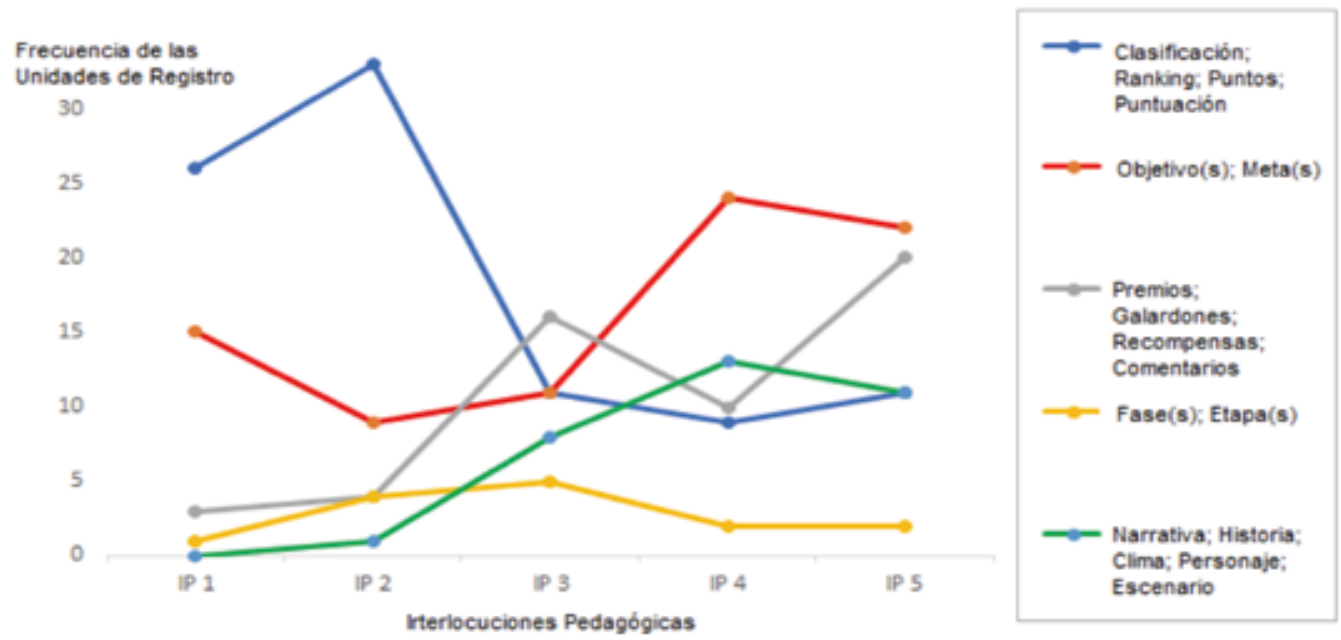

\section{Elaboración propia}

El gráfico muestra que los PEFI sobrevaloraron los sistemas de puntuación en detrimento de los demás elementos potenciales de gamificación al principio de las interlocuciones. A lo largo de los seminarios se mejoró el establecimiento de objetivos claros y cohesionados en las clases. La presencia de las narrativas como ejes conductores de la experiencia docente y como elementos simbólicos responsables de provocar el compromiso en el alumnado no fue utilizada por los PEFI inicialmente. Sin embargo, fue uno de los elementos de los juegos que más se utilizó 
al final de la experiencia, lo que parece indicar que este componente fue cobrando sentido, se comprendió mejor y se movilizó en el contexto de las clases gamificadas, como indican los pasajes destacados:

PEFI 11: Me pareció genial el lanzamiento de la historia con la lava en la clase... Está presente en muchos juegos y es una forma diferente de darle más dinámica al juego. (T4, 27/11/2019)

PEFI 15: El recurso de esta última clase, de los grupos captando las partes críticas a lo largo del camino, que recoges y forman las llaves para abrir un cofre y encontrar dentro esa historia del virus zombi, fue muy bueno. [...]. (T4, 27/11/2019)

El uso de metas y objetivos coherentes que guíen las acciones, y diversos sistemas de retroalimentación, no centrados en la mera competencia o cuantificación por calificación/puntos, sino a través del uso de insignias, premios, recompensas, fueron también elementos de gamificación que prácticamente no aparecían en las primeras clases, pero que se estaban asociando a las situaciones de enseñanza del Parkour de forma creativa y cada vez más frecuente. Según uno de los PEFI incluidos:

PEFI 10: Nos guste o no, hasta entonces estábamos trabajando en todas las clases con el enfoque que sería el ranking, que sería la puntuación, [...] me pareció genial ahora el feedback de las actividades que se hacían... [...] Lo que vemos en la gamificación es que cada juego es un problema, un reto a superar, a conquistar, una meta. [...] Creo que dejaron muy claro eso con la idea de rescatar la bandera. [...] Otra mecánica que me pareó genial, la que ustedes utilizaron, es la de repartir las vidas, ¿no? [...] Las bonificaciones dispersas. (T4, 27/11/2019)

Finalmente, el recurso de las fases o secciones de aprendizaje también estuvo presente desde el inicio de los seminarios, sin embargo, de forma más estable y sutil, sin mucho énfasis, pues cada una de las lecciones creadas por el PEFI constituía la adquisición de un nivel más de complejidad del tema desarrollado.

Comprobamos que los alumnos y las alumnas entendieron, durante los seminarios prácticos, el proceso de gamificación de forma más amplia, más allá del establecimiento de sistemas de disputa/competencia entre individuos y equipos, que avanzan etapas y son recompensados con regalos y puntos. Estos componentes pueden imponerse en los procesos de gamificación, pero no los aseguran. Por el contrario, pueden reafirmar formas y estrategias didácticas convencionales de sistemas tradicionales de enseñanza, como el énfasis en las actividades sin propósitos educativos contextualizados; la sobrevaloración del sistema de progresión lineal (fase o serie, años escolares, ciclos, etc.), y la objetivación de acciones a través de retroalimentaciones estrictamente cuantificadas - calificaciones que ocupan el lugar del fin (en el doble sentido que utiliza la palabra - ya sea como circunstancia que termina con otra o aún como sinónimo de motivo, razón de ser; objetivo hacia el que se tiende; intención).

En este sentido, Santaella et al. (2020) señalan que los procesos de gamificación a veces limitan la propia noción de juego a un enfoque conductista: como estrategia para motivar a los individuos y aumentar la productividad, ya sea en las relaciones empresariales o educativas.

Encontramos, por lo tanto, que el proceso de aprendizaje fue impulsado centralmente por la experiencia (Bondía, 2002; Schell, 2011) con la creación y la experiencia gamificada, en la que la presencia, el énfasis o la ausencia de ciertos elementos del juego actuaron como reveladores de la comprensión que el grupo de PEFI estableció respecto a la gamificación. La experiencia ayudó a los PEFI a aprender, convirtiéndose en un elemento para dilucidar las ideas, haciendo perceptibles los conceptos en su materialidad, sorteando la abstracción que a veces resulta del aprendizaje textual solo en la formación docente. 


\subsection{Interfaces entre gamificación, TDIC y cultura digital}

Otro núcleo de significado encontrado se refiere a la presencia de las Tecnologías Digitales de la Información y la Comunicación (TDIC) en la experiencia. En cuatro de las cinco clases del seminario práctico, los grupos de profesores del PEFI utilizaron teléfonos móviles, aplicaciones digitales, geolocalización, mapas digitales, filmación y registro fotográfico de las actividades y las clases, estableciendo una interfaz consistente entre el proceso de gamificación y los artefactos de la cultura digital.

Para los PEFI, el uso de las TDIC se consideró fundamental para lograr prácticas y acciones gamificadas porque estos recursos ayudaban a construir un ambiente, una estética de juego, remitiendo a la construcción simbólica de lo que Huizinga (1996) llamó el círculo mágico. De este modo, a medida que se utilizaban recursos específicos, se amplificaba la sensación de estar inmerso en un entorno de juego. En este sentido, se recogieron discursos como:

PEFI 08: Vaya, con esta musiquita de móvil [énfasis añadido], esto se está pareciendo incluso a uno de esos pequeños videojuegos que le gustan a mi hija. (T5, 12/11/2019)

PEFI 02: Realmente, el uso de los teléfonos móviles y los códigos QR [énfasis añadido] fue genial. (T1, 11/05/2019)

¡PEFI 03: Mi evaluación con respecto a tu clase de ayer, [...] Hay elementos muy interesantes que trajiste, recursos de gamificación... el tema de los mapas de Google Earth [...] la característica de las cámaras [énfasis añadido] que me pareció muy buena! Podíamos ver a la gente repitiendo las maniobras, [...] todo el mundo tenía la sensación de estar jugando un juego real. (T1, 11/05/2019).

Los discursos muestran que la presencia y el uso de las TDIC en los seminarios prácticos, en muchos pasajes, estuvieron asociados a contextos de valorización de estas tecnologías en la constitución de acciones gamificadas. Eso no quiere decir que las situaciones gamificadas solo puedan promover más inmersión y compromiso en sus participantes si se apoyan en el uso y la presencia de las TDIC. Como se señala en el estudio de Pimentel et al. (2020), reconocemos que realizar una propuesta gamificada no significa utilizar juegos digitales o Tecnología Digital (TD) en contextos de aprendizaje. Como dicen los autores, "la gamificación puede ser desarrollada sin el uso de artefactos digitales" (Pimentel et al., 2020, p. 8). Sin embargo, en nuestro estudio, tales recursos ocuparon un lugar de ambientación, de producción de sentido, de asociación creativa, más allá de su dimensión más instrumental, promoviendo lo que Brown y Cairns (2004) llamaron una gradación de la experiencia de inmersión en el ámbito de los juegos, en la que los jugadores pasan gradualmente de una situación de poca dedicación al juego al engagement (compromiso), al engrossment (ensimismamiento) y al total immersion (inmersión total).

Nuestros datos atestiguan que la asociación de acciones gamificadas al uso de las TDIC ha mejorado la perspectiva de la educación en la cultura digital durante la formación de los profesores y las profesoras. Al fin y al cabo, como reconoce Pimentel (2018, p. 78), la gamificación puede "involucrar y motivar a las personas, con el objetivo de aprender a través de las interacciones entre las personas, con las tecnologías [énfasis añadido] y con el entorno". Adoptando, por lo tanto, lo que Pimentel et al. (2020, p.10) consideran una articulación entre la gamificación y "el concepto de tecnología en una visión crítica de la relación hombre-tecnología".

Un ejemplo puede extraerse del primer seminario temático, que exploró la gamificación y su relación con la cultura digital a través de los teléfonos móviles y los códigos QR. ${ }^{1}$ El objetivo de la clase era introducir, de forma inicial, la modalidad de Parkour. Para ello, en el entorno de una pista polideportiva, se esparcieron varios códigos QR creados por el grupo de instructores 
del PEFI y se colocaron en lugares por los que los jugadores debían moverse utilizando algunas habilidades básicas requeridas en el deporte, como escalar, saltar obstáculos, etc. para acceder a ellos en un tiempo determinado. Para llegar a los códigos $\mathrm{QR}$, los participantes tenían que explorar e interactuar con el espacio, superando los obstáculos con movimientos corporales de forma creativa, ágil y segura.

Los códigos QR contenían información, como curiosidades, reglas, hechos históricos y descripciones de movimientos específicos sobre el Parkour. Esta actividad con las TDIC estaba asociada a la gamificación, ya que requería la formación de grupos. Había un reto/objetivo que cumplir (recoger la mayor cantidad de información posible en menos tiempo). Su resultado implicaba un premio y una clasificación, además de configurarse como una primera fase (de un juego-fase previa de reconocimiento de la modalidad de Parkour) que se extendería durante las siguientes cuatro clases posteriores.

Sin embargo, la acción pedagógica puso de manifiesto la oportunidad que la asociación entre gamificación y TDIC puede promover en términos de formación para y con la cultura digital. Este hecho puso de manifiesto que muchos PEFI, a pesar de utilizar smartphones (teléfonos inteligentes), no conocían la aplicación de los códigos $\mathrm{QR}$, ni su posibilidad de uso creativo o pedagógico. Así, fue una acción que incentivó la creación pedagógica, el conocimiento de las características e historia de esa aplicación, y, sobre todo, abrió la posibilidad de autoría con esa TDIC en función de una enseñanza más creativa del componente curricular Educación Física su asociación con estrategias gamificadas.

\subsection{Relación teórico-práctica en debate}

En nuestra última categoría, agrupamos los registros relativos a los debates de evaluación realizados en cada seminario temático. Una experiencia intersubjetiva que se mostró relevante en cuanto a la constitución de una comprensión de la gamificación articulada a los significados producidos (Lima \& Andrade, 2018) en el momento de las interlocuciones pedagógicas.

Como sugiere Mendes (2016), los seminarios prácticos fueron concebidos a la luz de una actitud pedagógica centrada en la interlocución. Esta palabra designa "un compartir múltiple, una comunicación, una conversación, un diálogo, y que presupone la existencia de sujetos que se comunican a partir de situaciones concretas en las que se encuentran" (Mendes, 2016, p. 184). En este sentido, muchos debates, permeados por la revisión de los fundamentos teóricos de la gamificación, así como de los textos esenciales estudiados en la asignatura, permitieron reflexionar sobre puntos en los que los PEFI manifestaban dificultad de comprensión, o incluso contradicciones teórico-prácticas, como se puede ver en algunos de los pasajes destacados:

PEFI 04: Yo quería hablar [...] La parte que hicieron un circuito...ah, así, no se podía ni ver, sentir, que estábamos en un juego. No sé; ni siquiera estaba relacionado con la lección anterior.

PEFI 05: Pero era un circuito. Esta parte no debía ser gamificada [énfasis añadido], solo en el momento del reto. Tanto es así que ni siquiera tenía puntos [énfasis añadido].

PEFI 08: Pero a eso se refiere. La gamificación no es solo hacer una actividad con puntos, con la competencia. Eso ya se hace en el aula tradicional. Lo que queremos decirle a su grupo es que tiene que pensar en el proceso, ¿sabe? [...] Cómo vas a hacer que el alumno aprenda, pero con un objetivo, que tiene que ser una meta, y que [...] luego lo vas a llevar a un objetivo, pero a través de todo, lo que es un juego... es el personaje, cobrando vida, ¿sabes? (T2, 08/11/2019)

En la comunicación anterior, la comprensión de la PEFI 05 sobre la gamificación todavía no estaba clara en términos de procedimiento. Para ella, algunas actividades podían ser gamifi- 
cadas; otras no. Su comprensión parecía reducir la gamificación a la presencia de tal o cual actividad en la escena pedagógica. A través de los contraargumentos de otros PEFI y de la profesora, esta comprensión pudo ponerse en tensión, abriendo espacio para la elaboración de nuevas asociaciones en relación con el procedimiento implicado en las acciones gamificadas.

En otro debate, algunos PEFI cuestionaron el énfasis de la literatura en predecir que la gamificación puede promover o fomentar el compromiso y la motivación de sus participantes. Durante el debate evaluativo del tercer seminario temático, el PEFI 14 cuestiona a la clase sobre la motivación que habían producido los demás participantes. El académico señala que, a pesar de que hasta ese momento esa era la clase que mejor se caracterizaba en cuanto a los supuestos de la gamificación, muchos participantes no se habían comprometido.

PEFI 14: Ellos [el grupo] utilizaron bien la gamificación. Creo que fue el grupo que más utilizó la gamificación. [...] Pero creo que un punto... [...] es que a los alumnos les faltó un poco de motivación. No sentí que estuvieran $\tan$ motivados. Porque creo que ese es uno de los elementos que intenta aportar la gamificación. Quería saber por parte del grupo lo que pensaban. (D2, 13/11/2019)

El debate reveló un ejercicio de cuestionamiento crítico por parte del PEFI para indagar sobre las proposiciones conceptuales estudiadas. Esta acción parece ser fundamental en la formación del profesorado, como ya recomiendan diferentes pensadores alineados con el concepto de formación reflexiva del profesorado (Nóvoa, 1989; Zeichner, 1993; Pimenta \& Ghedin, 2003). El PEFI argumentó que las acciones gamificadas pueden o no promover la motivación y el compromiso de los y las estudiantes para las acciones deseadas, aunque reconoció que la presencia de elementos de diseño de juegos tiene un potencial muy atractivo cuando tiene una buena arquitectónica.
Se plantearon las siguientes hipótesis para ayudar a entender por qué el alumnado no se sentía motivado por la experiencia gamificada: i) se consideró que esto podría ser un hecho ocasional, o incluso cuestiones externas que escapan al ámbito de la planificación de la gamificación, como las horas de clase después de la comida, el calor temporal del día y, en definitiva, que no era una cuestión inherente a la gamificación; ii) también se consideró que algunos elementos de la gamificación entran en conflicto con la cultura escolar, por ejemplo, en lo que respecta a la libertad de acción. En este contexto, se argumentó que los juegos son desarrollados por los jugadores de forma libre, voluntaria y deliberada y que los propios jugadores eligen los juegos que quieren jugar, el momento del día y la hora del mismo, el número de veces que se repiten, etc. Sin embargo, las acciones típicamente presentes en la cultura escolar y en los planes de estudio no permiten o incluso carecen de las condiciones para que la libertad de acción y la voluntariedad se experimenten plenamente.

Finalmente, notamos que los ejercicios evaluativos-reflexivos ganaron más consistencia conceptual a lo largo de los seminarios prácticos, pasando de una ausencia casi total de interlocuciones con las lecturas sugeridas en el período anterior del curso o incluso en las encuestas realizadas por los PEFI sobre el tema estudiado, a una presencia más significativa de análisis teóricos y prácticos al final de la experiencia. Como ejemplo, traemos una evaluación realizada por un PEFI en el último seminario práctico, que presenta estas características argumentativas:

PEFI 16: He leído los textos [...] Lo he releído, [el texto] de Mauro Berinbau, que es: "Gamificación: una propuesta de desarrollo basada en el diseño de juegos, con un enfoque en la comunicación". [...] Él habla de la estructura piramidal del juego, que es el establecimiento de las reglas, la libertad de acción y la condición de la experiencia para el jugador. Y luego el tema de Zombie fue la construcción de la experiencia.... [...] había el establecimien- 
to de reglas. [...] Había un pequeño mapa, había una ruta que podías tomar... Si querías ir al RU [restaurante universitario] podías ir. Es la libertad. [Risas]. Sobre la división [de los equipos]: los dividías en corredores, apoyo y guionista. [...] Recuerdo el texto; Barklei habla de la matriz de generación de comportamientos, que dividirá en corredores, asesinos, socializadores y exploradores. Pude entender que los corredores eran un poco conquistadores y asesinos. [...] Luego está el apoyo, que estaba filmando, ¿no? [...] Luego estaba el que filmaba, que tenía que prestar atención al otro de su equipo [...]. Y el guionista que tenía el mapa, ¿no? Yo lo veía como un explorador. Porque la característica del explorador es [PEFI hace la lectura]: "el deseo con la interacción del mundo del juego y sus posibilidades, buscando la sorpresa de la novedad, a través del descubrimiento de nuevos lugares, criaturas, objetos". (T5, 12/04/2019)

Notamos que la reflexión se reposicionó en un campo más teleológico y epistémico al final del proceso. En este sentido, pudimos notar que la experiencia forjó nuevas posibilidades de significación y aprendizaje al tomar la comunicación y la reflexión como elementos mediadores de las acciones producidas. Por lo tanto, si consideramos que el juego es siempre un acto dialógico, a veces colectivo y colaborativo, el aprendizaje sobre la dinámica de los juegos y su transposición a otros contextos no lúdicos también parece serlo.

\section{Conclusiones}

Los resultados demuestran la importancia de la formación en gamificación en la formación del docente. Los resultados revelaron la presencia de tres elementos relevantes para que los y las docentes se apropien de la gamificación: la comprensión del proceso de gamificación de forma más amplia, más allá de los sistemas de disputa/ competencia entre individuos y equipos; la asociación entre gamificación y TDIC puede promover la formación para y con la cultura digital; la presencia de ejercicios evaluativos-reflexivos, ya que permitieron a los PEFI reevaluar el proceso pedagógico, haciéndoles comprender el potencial del uso de la gamificación en la enseñanza.

Así, las conclusiones corroboran otros estudios ya realizados en el área y señalan el potencial de la gamificación en la formación docente (Alves et al., 2014; Carvalho \& Lima, 2019; Martins \& Giraffa, 2015).

Consideramos relevante que las acciones de formación docente para y con gamificación contemplen, siempre que sea posible, ejercicios teórico-metodológicos de creación y experiencia práctica de situaciones gamificadas, centradas en la práctica docente, ya que ayudaron al desarrollo de una mayor consistencia conceptual y a la capacidad de justificar de forma más cohesionada y científica las acciones didácticas y la planificación pedagógica relativas a la gamificación.

\section{Notas}

\begin{abstract}
El parkour es un deporte contemporáneo de práctica corporal, de origen francés, cuyo objetivo es recorrer un trayecto, superando cualquier obstáculo de forma rápida, segura y eficaz, solo utilizando las habilidades y capacidades del cuerpo humano.

El código QR es un código de barras bidimensional, o código barramétrico, que se puede escanear fácilmente con la mayoría de los teléfonos móviles equipados con cámara y que es capaz de retener y permitir el acceso a diferentes informaciones.
\end{abstract}

\section{Referencias bibliográficas}

Alves, F. (2015). Gamificação: Como criar experiencias de aprendizagem engajadoras. DVS Editora.

Alves, L. R. G., Minho, M. R. da S., \& Diniz, M. V. C. (2014). Gamificação: Diálogos com a educação. In FADEL, Luciane Maria, Ulbricht, Vania Ribas, Batista, Claudia Regina; Vanzin, Tarcísio, Gamificação na Educação. Pimenta cultural.

André, M. E. D. A. D. (2005). Estudo de caso em pesquisa e avaliação educacional. Líber livro.

Apostol, S., Zaharescu, L., \&Alexe, I. (2013). Gamification of learning and educational games. Elearning \& Software For Education, (2).

Bardin, L. (2016). Análise de conteúdo. Edições 70.

Bogdan, R., \& Biklen, S. (1994). Investigação qualitativa em educação: uma introdução à teoria e aos métodos. Porto editora. 
Bondía, J. L. (2002). Notas sobre a experiência e o saber de experiência. Revista brasileira de educação, (19), 20-28.

Carvalho, D. A. C., \& Lima, Marcio Roberto de. (2019). Percepções e Possibilidades da Inserção de uma Estratégia Gamificada na Formação Inicial de Professores. Anais do XVIII SBGames, Rio de Janeiro, Brasil. https://bit.ly/3Iz05W0

De Barros, P. M. (2019). Cultura digital e a formação de professores: o ensino de história no tempo presente. InterMeio: Revista do Programa de Pós-Graduação em Educação-UFMS, 25(49.1). https://bit.ly/31Ln8fn

Deterding, S., Khaled, R., Nacke, L. E., \& Dixon, D. (2011). Gamification: Toward a Definition In CHI 2011 Gamification Workshop Proceedings, Vancouver, BC, Canada.

https://bit.ly/3lLOMQw

Fardo, M. (2013). A gamificação como método: estudo de elementos dos games aplicados em processos de ensino e aprendizagem. (Dissertação de mestrado). Universidade de Caxias do Sul, Caxias do Sul, Rio Grande do Sul, Brasil.

Huizinga, J. (1996). Homo ludens. Editora Perspectiva SA.

Kapp, K. M. (2012). The gamification of learning and instruction: game-based methods and strategies for training and education. John Wiley \& Sons. KIM, A. J. Gamification 101: Designing the player journey. Google Tech Talk, 2011.

Lemos, A. (2020). Epistemologia da comunicação, neomaterialismo e cultura digital. Galáxia (São Paulo), (43), 54-66.

https://bit.ly/3dtLVHr

Lemos, A., \& Lévy, P. (2010). O futuro da internet: em direção a uma ciberdemocracia planetária.: Paulus, 13.

Lévy, P. (2007). Inteligência coletiva (A). Edições Loyola.

De Lima, M. R. (2013). Web 2.0: plataforma para la reconfiguración de la educación en la cibercultura. Alteridad, 8(1), 144-154. bit.ly/3ybp1hr

Lima, M. R. de, \& Andrade, I. M. de. (2018). Significado que los docentes le dan a la integración de tecnologías digitales en sus prácticas pedagógicas. Alteridad, 14(1), 12-25.

https://doi.org/10.17163/alt.v14n1.2019.01

Martins, C., \& Giraffa, L. M. M. (2015). Gamificação nas práticas pedagógicas em tempos de ciber- cultura: proposta de elementos de jogos digitais em atividades gamificadas. XI SJEEC Seminário Jogos Eletrônicos, Educação e Comunicação, 2015, Brasil.

McGonigal, M. (2012). J. A realidade em jogo: por que os games nos tornam melhores e como eles podem mudar o mundo. BestSeller.

Mendes, D. S. (2016). O estágio na licenciatura em educação física em perspectiva semiótica:(re) ver-se e (re) criar-se em imagens. $244 \mathrm{f}$. Tese (Doutorado em Educação). Universidade Estadual Paulista "Júlio de Mesquita Filho", Presidente Prudente/SP, 2016.

Minayo, M. C. D. S. (2012). Análise qualitativa: teoria, passos e fidedignidade. Ciência \& saúde coletiva, 17, 621-626. https://bit.ly/31EMD27

Nanodome (2011). The (short) prehistory of "gamification"... Funding Startups (\& Other Impossibilities). https://bit.ly/3rVkoqu

Nóvoa, A. (1989). Profissão: professor. reflexões históricas e sociológicas. Análise Psicológica, 7, 435-456. https://bit.ly/3oAHXTJ

Pimenta, S. G., \& Ghedin, E. (2003). Professor reflexivo no Brasil: gênese e crítica de um conceito. Cortez Editora.

Pimentel, F. S. (2018). Considerações do planejamento da gamificação de uma disciplina no curso de Pedagogia. In FOFONCA, Eduardo et al. (Org.), Metodologias pedagógicas inovadoras: contextos da educação básica e da educação superior (v. 1. pp. 76-87). Editora IFPR.

Pimentel, F. S. C., Nunes, A. K. F., \& Sales Júnior, V. B. D. (2020). Formação de professores na cultura digital por meio da gamificação. Educar em Revista, 36. https://bit.ly/3pCwBhk

Santaella, L., Nesteriuk, S., \& Fava, F. (Eds.). (2018). Gamificação em debate. Blucher.

Schell, J. (2011). Arte de game design: o livro original. Elsevier.

Werbach, K., \& Hunter, D. (2012). For the win: How game thinking can revolutionize your business. Wharton Digital Press.

Yin, R. K. (2015). Estudo de Caso: Planejamento e métodos. Bookman editora.

Zeichner, K. M. (1993). A formação reflexiva de professores: idéias e práticas. Educa.

Zicherman, G., \& Cunningham, C. (2011). Gamification by design. Sebastopol. 


\section{ALERIDAD Gamificación y aprendizaje colaborativo en línea: un análisis de estrategias en una universidad mexicana \\ Gamification and collaborative online learning: an analysis of strategies in a Mexican university}

Dr. William Reyes-Cabrera es profesor e investigador de la Universidad Autónoma de Yucatán (México) (wreyes@correo.uady.mx) (https://orcid.org/0000-0002-3443-6385)

Recibido: 2021-09-19 / Revisado: 2021-12-01 / Aceptado: 2021-12-13 / Publicado: 2022-01-01

\section{Resumen}

Aunque la educación a distancia ha demostrado sus beneficios en el proceso de enseñanza, una de sus principales carencias es la falta de estrategias propias; sin embargo, la gamificación se considera como una herramienta efectiva para trabajar en esta modalidad. Por ello, se realizó una investigación de enfoque cuantitativo, de alcance exploratorio del tipo preexperimental en una universidad mexicana con los siguientes objetivos: identificar el nivel de incidencia que tienen las estrategias de gamificación en el aprendizaje colaborativo en un curso a distancia desde la perspectiva del estudiantado universitario; y comprobar si existen diferencias significativas entre las diferentes estrategias de gamificación en el aprendizaje colaborativo del estudiantado universitario. Se utilizó un curso a distancia donde se emplearon tres estrategias de gamificación (Tríada PET, Digital StoryTelling y Escape Room); el alumnado contestó el instrumento COLLES al finalizar cada una de estas para contrastar las respuestas obtenidas, por lo que se compararon las medias, desviaciones estándar y la ANOVA de un factor para muestras repetidas. Los resultados indicaron que las tres estrategias incidieron en el aprendizaje colaborativo, aunque existieron diferencias significativas en sus escalas. Se concluye que las características de cada estrategia son determinantes para lograr el aprendizaje colaborativo en línea. Se recomienda seleccionar y articular las estrategias de gamificación en el diseño de un curso a distancia y realizar mediciones constantes para identificar y mantener los niveles de aprendizaje colaborativo.

Descriptores: Educación, medición, aprendizaje, colaboración, gamificación, estrategia.

\section{Abstract}

Despite distance education has proved its benefits in the teaching process, one of its main shortcomings is the lack of its own strategies, however, gamification is considered as an effective tool to work with on this modality. Based on the above, a pre-experimental, exploratory, quantitative research study was carried out in a Mexican university following the next objectives: identifying the level of incidence that gamification strategies have regarding collaborative learning in a distance course from the perspective of university students; as well as checking if there are significant differences between the different gamification strategies in collaborative learning of university students. A distance course was used, for this purpose where three gamification strategies were performed (PBL, Digital StoryTelling and Escape Room); students answered then the COLLES instrument at the end of each of these strategies to find contrast to the responses obtained. Thus, the means, standard deviations and ANOVA of one factor for repeated samples were compared. The results indicated that all three strategies had a positive impact on collaborative learning, however, there are significant differences in their scales. It is concluded that the characteristics of each strategy are key in achieving online collaborative learning. It is recommended to select and articulate gamification strategies when designing a distance course considering constant measurements to identify and maintain the levels of collaborative learning.

Keywords: Education, measurement, learning, collaboration, gamification, strategy.

Forma sugerida de citar: Reyes-Cabrera, W. (2022). Gamificación y aprendizaje colaborativo en línea: un análisis de estrategias en una universidad mexicana. Alteridad, (17)1, 24-35. https://doi.org/10.17163/alt.v17n1.2022.02 


\section{Introducción}

La educación a distancia $(\mathrm{EaD})$ ha tomado mayor relevancia en los últimos años, impulsada principalmente por la evolución de la tecnología, los cambios sociales, económicos y recientemente, como alternativa para continuar con las actividades cotidianas desde los hogares, como consecuencia de la pandemia que aqueja a la humanidad. Además, ha demostrado sus ventajas frente a la educación presencial, principalmente por la flexibilidad en los horarios para trabajar, en el acceso a los recursos para la realización de las tareas, así como del soporte tecnológico que es más asequible tanto para las instituciones como para la mayoría del estudiantado (Pattanshetti et al., 2018). Otras ventajas son el grado de experiencia que tienen los profesores, las profesoras y estudiantes en el manejo de las plataformas educativas para las actividades sincrónicas y asincrónicas, además de contar con la aceptación social ya generalizada de esta modalidad (Teo et al., 2020), lo que permite que esté presente en todos los niveles educativos. Sin embargo, se ha observado carencias en la planificación y ejecución de estrategias de enseñanza y aprendizaje pertinentes para la EaD (Al-Jedaiah, 2020), lo que ha originado la traslación de estrategias de la educación presencial a la virtualidad o un "reempaquetado de estrategias de instrucción tradicionales" (Wiggins, 2016, p. 27), así como las crecientes preocupaciones en la poca socialización que tiene el alumnado al no interactuar físicamente con otros compañeros/as, incrementado ahora por las restricciones de movilidad actuales, lo que abona a la deserción, abandono de los estudios, e incluso a problemas emocionales y psicológicos (Boverman \& Bastiens, 2020).

Frente a estos escenarios es indispensable contar con estrategias que permitan la motivación y el interés por continuar con los estudios formales en la $\mathrm{EaD}$, fundamentalmente para mejorar las condiciones de aprendizaje, más allá de ser considerado como un aprendizaje basado en la tecnología (Teo et al., 2020), para lograr experien- cias de aprendizaje únicas, con una amalgama de herramientas digitales y estrategias propias para la $\mathrm{EaD}$ (Barna \& Fodor, 2019). Existen diversas estrategias y metodologías abocadas a lograr estas nuevas formas de aprender en la virtualidad, siendo la gamificación una herramienta efectiva en el aprendizaje a distancia y en línea (Rebelo \& Isaías, 2020) ya que puede motivar y comprometer a los estudiantes para continuar con el proceso de formación (Poondej \& Lerdpornkulrat, 2019), potenciando las experiencias de las nuevas generaciones en todos los niveles educativos al emplear este tipo de estrategias y los recursos tecnológicos actuales (Duggal et al., 2021).

\subsection{La gamificación y sus estrategias}

La gamificación es la "utilización de elementos de diseño de juegos en contextos no lúdicos" (Deterding et al., 2011, p. 2425); un método (Rodríguez \& Arias, 2020) orientado hacia la motivación (Laine \& Lindberg, 2020), que puede inducir cambios en el comportamiento y fortalecer el compromiso de los participantes (Hamari et al., 2016); sin embargo, la aplicación superficial de la gamificación considerando solo los aspectos básicos del juego perdiendo de vista el carácter pedagógico y del aprendizaje, hace que la gamificación pueda generar frustración y aburrimiento (Khoshkangini et al., 2021) por lo que se recomienda utilizar diversas estrategias para mantener los niveles de atención y motivación. Al respecto, Kapp et al. (2014) señalan que la estructura de los eventos de gamificación o "eventos interactivos de aprendizaje" (p. 2) deben ser enfocados al contenido de las unidades de aprendizaje sin perder las experiencias que generan los juegos. Por su parte, Kingsley y Grabner-Hagen (2018) afirman que todo el curso, tanto en el vocabulario, como en las mecánicas empleadas y el diseño de los componentes deben estar orientados al juego; mientras que Werbach y Hunter (2015) señalan que una actividad se considere como de gamificación se deben emplear tres o más mecánicas. 
Aunque la gamificación no tiene estrategias propias, existen varias que por sus características se vinculan con esta (Silva et al., 2019). Por ejemplo: una de las estrategias más utilizadas es la tríada PBL [Points, Badges and LeaderBoards] o en español, PET: Puntos, Emblemas y Tablas de Clasificación. PBL es una estrategia básica en la gamificación que consiste en emplear tres mecánicas: un sistema de puntos para los participantes por la realización de una actividad; los emblemas o insignias que son premios o reconocimientos por alcanzar un logro en alguna de las actividades de manera sobresaliente; y las tablas de clasificación, consideradas como un "ranking"que se genera de acuerdo con el puntaje y emblemas obtenidos por los participantes (Werbach \& Hunter, 2015; y Fernandes et al., 2018). Otra de las estrategias más utilizadas es el StoryTelling o Digital StoryTelling si se usa recursos digitales (Buendgens, 2021), donde se desarrolla la narración de una historia que involucra a los participantes, tomando el rol de los personajes de dicha historia. Esta narración está dividida en capítulos o episodios que van transcurriendo en la medida que los participantes tomen decisiones para la progresión y finalización de estos. (Lawrence \& Paige, 2016; Gambarato \& Dabagian, 2016). Finalmente, la estrategia de EscapeRoom (Fotaris et al., 2016; y Bartlet \& Anderson, 2019) conocido en español como Cuarto de Escape o Breakout en inglés, consiste en tener a un grupo de personas en una habitación cerrada, en la que, para salir, deben resolver una serie de pistas o acertijos asociados al tema de alguna asignatura. En los cursos a distancia, las habitaciones y los acertijos son generados digitalmente y los participantes deben resolverlos de manera colaborativa mediante sus dispositivos conectados a internet (Jiménez et al., 2020).

\subsection{El aprendizaje colaborativo a distancia}

El aprendizaje colaborativo es un enfoque educativo de la enseñanza y aprendizaje para que los estudiantes trabajen juntos en la resolución de un problema, completar una tarea o crear un producto (Laal \& Laal, 2012), esta forma de aprendizaje se ha empleado en la enseñanza presencial con buenos resultados (Laal \& Ghodsi, 2012) y en la $\mathrm{EaD}$ ha permitido desarrollarse exitosamente, principalmente con el apoyo de las tecnologías de información y comunicación (Al-Samarraie \& Saeed, 2018); sin embargo, el principal reto está en el profesorado y expertos al momento de diseñar las experiencias de aprendizaje y el rendimiento académico en un entorno en línea (Kumi-Yeboah, 2018). Para estudiar al aprendizaje colaborativo se han realizado distintas investigaciones, (SyedMohamad et al., 2006; Fraternali \& Herrera, 2019; Azhari et al., 2020), mismos que han permitido tener una serie de indicadores clave para identificar el aprendizaje colaborativo en línea, como el pensamiento reflexivo, la interacción, el apoyo del profesorado, los compañeros y compañeras, interpretación, relevancia, entre otros; a partir de los cuales se puede medir si se alcanzó el aprendizaje colaborativo en línea mediante las estrategias y materiales utilizadas en las unidades de aprendizaje en un curso $\mathrm{EaD}$ (Taylor \& Maor, 2000; Yen et al., 2018).

\subsection{Objetivos de la investigación}

Aunque se ha demostrado que la gamificación promueve la motivación y el compromiso por aprender, se necesita identificar cómo incide en el aprendizaje colaborativo. Por lo tanto, es necesario conocer el efecto de estas estrategias que son cada vez más utilizadas en los contextos educativos a distancia. A partir de lo anterior, se realizó una investigación con los siguientes objetivos principales:

O1: Identificar el nivel de incidencia que tienen las estrategias de gamificación en el aprendizaje colaborativo en un curso a distancia desde la perspectiva del estudiantado universitario.

O2: Comprobar si existe diferencias significativas entre las diferentes estrategias de gamificación en el aprendizaje colaborativo del estudiantado universitario en un curso a distancia. 


\section{Metodología}

\subsection{Diseño de la investigación}

La investigación realizada tuvo un enfoque cuantitativo de alcance exploratorio del tipo preexperimental (Hancock, 2004) ya que se trabajó con un grupo de estudiantes que cursaron una asignatura oficial a distancia en la que hubo una intervención utilizando tres estrategias de gamificación y que al finalizar cada una de ellas contestaron un instrumento estandarizado para identificar el nivel de aprendizaje colaborativo en línea.

\subsection{Estructura del curso a distancia}

La asignatura seleccionada para la investigación fue la de "Comunicación científica", se impartió en el ciclo escolar 2020-2021 en la Universidad Autónoma de Yucatán (UADY), en una licenciatura del área de las ciencias sociales con una duración de 60 horas, tres unidades de aprendizaje, todas con las mismas características en cuanto al número de horas asignadas, al nivel de pensamiento de sus objetivos (Pikhart \& Klimova, 2019), los productos a entregar, el número de temas, los recursos didácticos, las actividades y el número de asesorías. Este curso se imparte desde 2016 a distancia y se ha empleado diversas estrategias de gamificación como los retos, las insignias, los puntos, las narrativas, competencias, escape room, entre otros. Dada la experiencia adquirida en el empleo de estas estrategias, se decidió utilizar las tres que han sido mencionadas anteriormente, por lo que en el diseño del curso se consideró que en cada unidad de aprendizaje los y las estudiantes realizaran las actividades mediante una estrategia de gamificación diferente; también se eligió la estrategia en función de su complejidad, comenzando por la tríada PBL en la unidad I, luego el Digital StoryTelling en la unidad II y finalmente el Escape Room en la unidad III. En la Tabla 1 se presenta la descripción de cada estrategia y los objetivos empleados en cada unidad:

\section{Tabla 1. Relación de objetivos y estrategias empleados en el curso a distancia}

\begin{tabular}{|c|c|c|c|}
\hline & Objetivo de la Unidad & $\begin{array}{l}\text { Estrategia general } \\
\text { de la Unidad }\end{array}$ & Descripción general la estrategia usada \\
\hline I. & $\begin{array}{l}\text { Identificar la relevancia de las } \\
\text { tecnologías de información } \\
\text { para difundir el conocimiento } \\
\text { científico y tecnológico. }\end{array}$ & PBL & $\begin{array}{l}\text { Formados en equipos, presentarán diversos recursos tecnológi- } \\
\text { cos que permitan difundir el conocimiento científico. Si cumple } \\
\text { con los requisitos recibirán puntos, si es un trabajo sobresalien- } \\
\text { te, se les otorgará una insignia. La relación de puntos e insignias } \\
\text { se presenta en una tabla de clasificación de los equipos. }\end{array}$ \\
\hline II. & $\begin{array}{l}\text { Identificar los medios digitales } \\
\text { e impresos para la publicación } \\
\text { de resultados de investiga- } \\
\text { ción, como una forma de co- } \\
\text { municar la ciencia. }\end{array}$ & Digital StoryTelling & $\begin{array}{l}\text { A los equipos se le plantea un caso para difundir una investi- } \\
\text { gación en medios electrónicos. El caso irá avanzando por ca- } \\
\text { pítulos, la toma de decisiones del equipo permitirá continuar } \\
\text { con los siguientes capítulos hasta su finalización. }\end{array}$ \\
\hline III & $\begin{array}{l}\text { Elaborar materiales para } \\
\text { apoyar en forma oral auxi- } \\
\text { liado con proyecciones por } \\
\text { computadora. }\end{array}$ & Escape Room & $\begin{array}{l}\text { Los equipos de trabajo realizan una serie de materiales (pre- } \\
\text { sentación electrónica, infografía y videotutorial) utilizando los } \\
\text { recursos que el instructor les indique. Cada material debe } \\
\text { hacerse en un lapso de un día como máximo para continuar } \\
\text { con el siguiente material. }\end{array}$ \\
\hline
\end{tabular}

\section{Fuente: Elaboración propia.}

Cabe señalar que el programa de la asignatura no se modificó para adaptarlo a las estrategias; las modificaciones se realizaron en el mate- rial entregado a los y las estudiantes, por ejemplo, en el lenguaje empleado y en el diseño gráfico de los recursos digitales, que se parecían más a un 
videojuego que a una tarea escolar (Kapp et al., 2014; Kingsley \& Grabner-Hagen, 2018; Sheldon, 2012), además las actividades y recursos de cada unidad debían estar orientados a cumplir con el objetivo de la unidad y al desarrollo de la estrategia correspondiente. Las herramientas tecnológicas utilizadas para la implementación del curso a distancia fueron la plataforma Moodle para las actividades asincrónicas como los foros de discusión, las tareas, enlaces a vídeos y materiales de lectura. Debido a las características técnicas de esta plataforma, la obtención de las insignias, desbloqueo de actividades y recursos fue de manera automatizada, lo que favoreció a enriquecer la experiencia con las estrategias de gamificación (Barna \& Fodor, 2019). Por otra parte, para las actividades sincrónicas como las sesiones de asesoría y tutoría, se empleó la plataforma de Microsoft Teams. En conjunto, por las características de ambas plataformas y las estrategias empleadas permitió que tener un curso completo basado en la gamificación para la $\mathrm{EaD}$.

\subsection{Participantes}

Fueron 90 estudiantes de la misma licenciatura y del mismo semestre que cursaron la asignatura, todos fueron invitados a participar voluntariamente en la investigación, explicándoles los objetivos, el alcance y que su participación no modificaba sus experiencias con las estrategias de gamificación ni sus calificaciones. Finalmente, participaron 85, de los cuales 45 fueron mujeres (53\%) y 40 hombres (47\%). Se aseguró que tuvieran acceso a los recursos tecnológicos, todos tenían acceso a un equipo de cómputo y a una conexión a internet; la mayoría $(n=66,78 \%)$ contaban con estos recursos en sus casas, mientras que 19 (22\%) se conectaban en otros lugares como la casa de un familiar o desde el trabajo.

\subsection{Instrumento}

Para la medición del aprendizaje colaborativo se utilizó la encuesta sobre el entorno de aprendi- zaje constructivista en línea o COLLES (Taylor \& Maor, 2000) por sus siglas en inglés. Este es un instrumento que permite evaluar la calidad de un entorno de aprendizaje a distancia desde la perspectiva del constructivismo social de Vygotsky (Dougiamas \& Taylor, 2002). El instrumento consta de 24 reactivos divididos en seis dimensiones o escalas: 1 . Relevancia, reactivos 1 al 4; 2. Pensamiento Reflexivo, del 5 al 8; 3. Interactividad, 9 al 12; 4 . Apoyo del tutor, 13 al 16; 5. Apoyo de los compañeros, 17 al 20; y 5. Interpretación, 21 al 24 (Yeo et al., 2006). La respuesta a los reactivos se realizó mediante una escala likert de cinco alternativas, donde 1 es "casi nunca" a 5 que es "casi siempre".

En relación con la confiabilidad y validez del instrumento, se obtuvo mediante el análisis de estudios similares donde se comprobó a través de los resultados de consistencia interna un coeficiente $\alpha$ de 0.80 (Yeo et al., 2006; Rivero, 2018; Azhari et al., 2020). Por su parte, Baker (2005) considera que las escalas son útiles tanto para los profesores como para los investigadores en la identificación de un aprendizaje colaborativo auténtico basado en la $\mathrm{EaD}$; y recientemente, Gutiérrez y Duche (2021) afirman que este instrumento permite la integración de un modelo para el aprendizaje colaborativo en línea en tiempos de confinamiento por la contigencia sanitaria provocada por el COVID- 19.

\subsection{Recogida y procesamiento de datos}

Al final de cada unidad de aprendizaje, todos los y las estudiantes respondían la encuesta COLLES disponible en la plataforma Moodle. Las respuestas se descargaron en archivos de formato .csv que sirvieron para integrar la base de datos. La siguiente figura muestra graficamente el proceso utilizado para obtener la información. 
Figura 1. Esquema de la recogida y procesamiento de datos

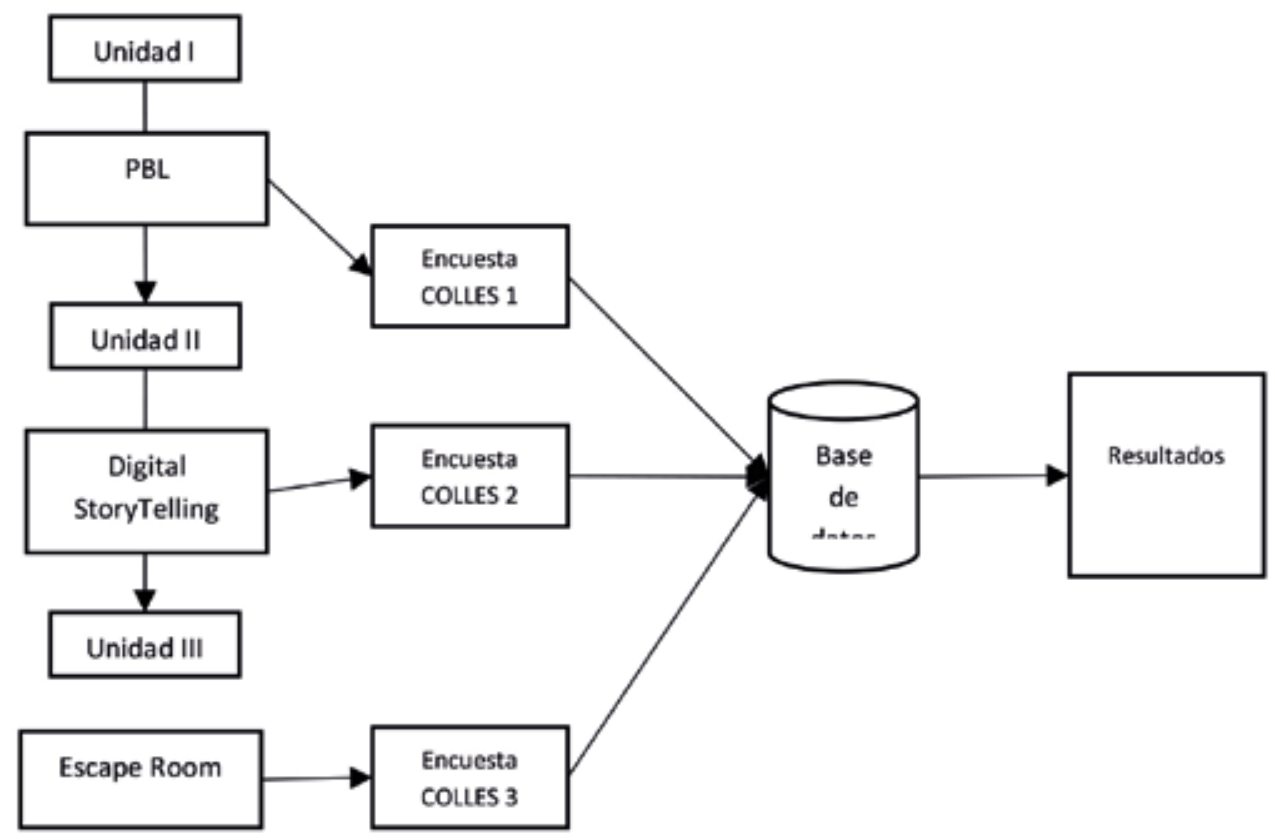

\section{Fuente: Elaboración propia}

Debido a la naturaleza de la investigación y de sus objetivos, se empleó el análisis y comparación de medias y desviaciones estándar, así como el uso de la ANOVA de un factor para muestras repetidas. Para considerar si hubo incidencia en el nivel de aprendizaje colaborativo, se tuvo como criterio que el puntaje promedio debería ser mayor a tres $(>3.0)$, en caso contrario $(<3.0)$ se considera que la estrategia no tuvo incidencia; este criterio también se aplicó para cada escala que compone el instrumento COLLES (Taylor \& Maor, 2000). El valor de significancia definido en las pruebas se consideró que sea de $0.05 \mathrm{y}$ el tamaño del efecto utilizada fue la eta parcial al cuadrado determinado en $>.06$ como un efecto mediano y $>.08$ un efecto grande (Kraft, 2020). El programa utilizado para la realización de las pruebas estadísticas y el procesamiento de la información fue el SPSS versión 24.

\subsection{Aspectos éticos}

Como se comentó anteriormente, todo el alumnado fue informado y aceptó que se publicaran sus respuestas. También se omitió intencionalmente el nombre de la licenciatura y de la facultad con el fin de garantizar el anonimato de los participantes. Esta información es conocida y aprobada por las autoridades de la facultad en la que se realizó la investigación.

\section{Resultados}

Una vez que se procesó la información colectada, se realizaron las pruebas de validez interna consistente en el Alpha de Cronbach y el Omega de McDonald; en el primero se obtuvo $\alpha=0.947 \mathrm{y}$ el segundo $\omega=0.949$. Luego, al de realizar las pruebas estadísticas señaladas en la metodología, se presentan los siguientes resultados: 


\subsection{Niveles de Aprendizaje Colaborativo}

Al obtener los puntajes de cada estrategia, se encontró que la tríada PBL tuvo una media de $(\mathrm{M}=4.17$, D.S. $=0.59)$, Digital StoryTelling $(\mathrm{M}=$
4.27, D.S. $=0.56)$ y Escape Room $(\mathrm{M}=3.97$, D.S. $=0.61)$.

Al realizar el procesamiento de los datos, se obtuvieron las medias de cada escala que conforman el Aprendizaje Colaborativo, cuyos puntajes se representan en la Figura 2 de la siguiente forma:

Figura 2. Comparación de las escalas que conforman el Aprendizaje Colaborativo entre las tres principales estrategias de gamificación utilizadas en el curso a distancia

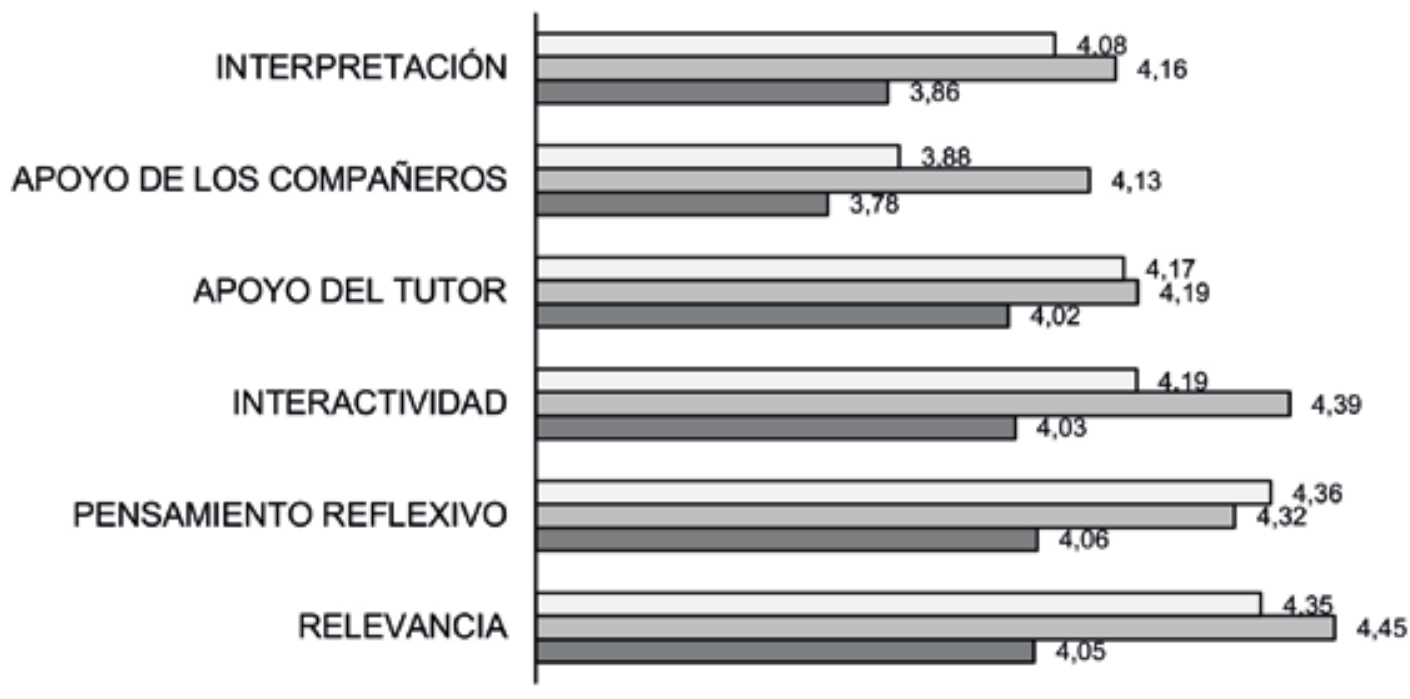

口PB $\quad$ Digital StoryTelling aEscape Room

\section{Fuente: Elaboración propia}

Se observa que en la mayoría de las escalas la estrategia de Digital StoryTelling obtuvo los mayores puntajes, especialmente en Interactividad y Relevancia, $\mathrm{M}=4.39$ y $\mathrm{M}=4.45$ respectivamente; por su parte, la estrategia de Escape Room tuvo los menores puntajes en todas las escalas analizadas. Adicionalmente, se encontró que, tanto en los niveles de aprendizaje colaborativo como en sus escalas, todas las estrategias tuvieron puntajes por encima del criterio establecido en la metodología.

\subsection{Diferencias de los niveles de aprendizaje colaborativo}

Al comprobar si existieron diferencias significativas en el nivel de aprendizaje colaborativo entre las tres estrategias de gamificación utilizadas en el curso a distancia, los valores obtenidos entre el nivel Aprendizaje Colaborativo con el tipo de estrategia tuvo un valor de $\mathrm{F}=4.008, \mathrm{p}=.021$ y $\eta 2 p=.065$, lo que indica que existe diferencias entre las estrategias empleadas; al realizar la prueba post-hoc de Bonferroni las diferen- 
cias fueron entre el Escape Room con Digital StoryTelling $(\mathrm{p}=.031, \eta 2 \mathrm{p}=.077)$.
Con relación a las escalas, se presenta la Figura 3 para representar gráficamente las diferencias entre las estrategias utilizadas.

Figura 3. Diferencias entre los puntajes obtenidos en las escalas que conforman el Aprendizaje Colaborativo
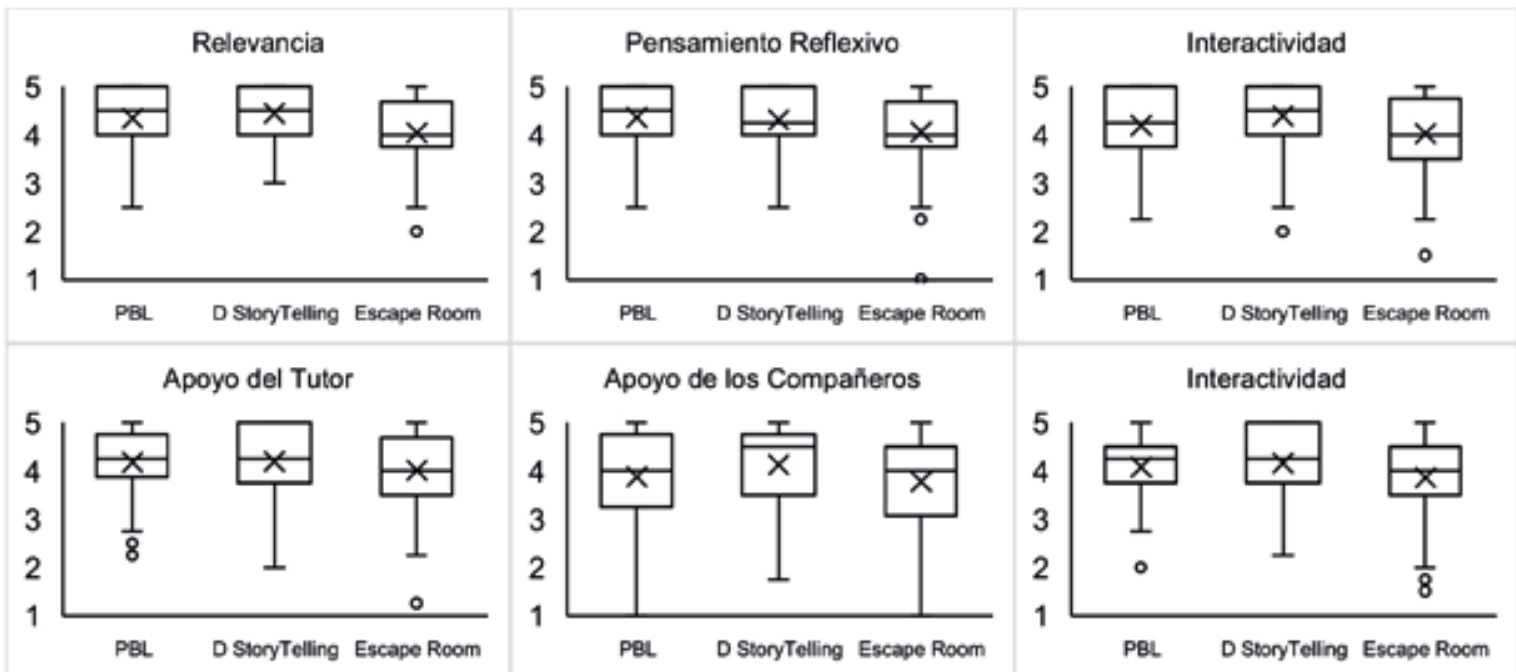

Fuente: Elaboración propia.

Se encontraron diferencias significativas en tres escalas: la de Relevancia $(\mathrm{F}=5.680, \mathrm{p}=0.04$, $\eta 2 \mathrm{p}=.082)$, Pensamiento Reflexivo $(\mathrm{F}=3.610$, $\mathrm{p}=0.029, \eta 2 \mathrm{p}=.071)$ e Interactividad $(\mathrm{F}=3.490$, $\mathrm{p}=0.033, \eta 2 \mathrm{p}=.060)$. Al realizar las pruebas post hoc de Tukey, en la escala Relevancia se encontraron diferencias significativas entre las estrategias de Escape Room y Digital StoryTelling (dif $=-.39$, p.=.004) y Escape Room con PBL (dif=-.29, p.=.040), en la escala Pensamiento reflexivo es Escape Room con PBL (dif=-.30, p.=.035) y la escala Interactividad fueron Escape Room con Digital StoryTelling (dif=-.36, $\mathrm{p}=.025)$.

\section{Discusión y conclusiones}

Inicialmente el curso a distancia se concibió para que los y las estudiantes realizaran todas las actividades en grupo; después, al emplear las estrategias de gamificación también se tuvo en cuenta el trabajo en equipos y apoyo entre los integrantes para el logro de las competencias declaradas en el programa de estudios. Lo anterior dio como resultado que los niveles de Aprendizaje Colaborativo sean altos, sin embargo, en el análisis de las escalas se encontraron diferencias como en el caso de las escalas de Relevancia e Interactividad cuyos puntajes fueron mayores en Digital StoryTelling, una estrategia que es ampliamente utilizada en la gamificación por los profesores, especialmente en el aprendizaje de idiomas y en las ciencias sociales (Silva et al., 2019) debido a que "permite que un mundo ficticio se enfrente de forma más eficaz a los retos que se presentan en el mundo real" (Gambarato \& Dabagian, 2016, p. 242) y que favoreció al vínculo teoría-práctica profesional en el aprendizaje en línea así como el establecimiento de un rico diálogo educativo (Taylor \& Maor, 2000).

Por su parte, la tríada PBL tuvo los mayores puntajes y diferencias significativas en la escala del Pensamiento Reflexivo, que es el desarrollo del pensamiento crítico que los y las estudiantes tienen sobre los contenidos vistos durante la unidad de aprendizaje (Dougiamas \& Taylor, 2002), además que fomentó el espíritu competitivo 
para obtener una mejor posición en el ranking de los equipos, como sucedió en el estudio de Uz y Gul (2020) al emplear esta estrategia. En el caso del Escape Room, también incidió en el Aprendizaje Colaborativo a distancia, sin embargo, al realizar las pruebas estadísticas y comparar sus puntajes con las otras estrategias, aquellas obtuvieron los mejores resultados; esto posiblemente se deba a que esta estrategia está orientada a desarrollarse en un medio físico, utilizando un salón de clase y los objetos reales (Bartlet \& Anderson, 2019) aunque existen diseños virtuales para el escape room cuyos resultados han sido satisfactorios (Jiménez et al., 2020; Hunt et al., 2020). Por lo anterior, se concluye que las estrategias de gamificación utilizadas en el curso a distancia tuvieron incidencia en el Aprendizaje Colaborativo en Línea, siendo la estrategia de Digital StoryTelling la que obtuvo mayor puntaje, posiblemente por las características de cada estrategia que serían factores determinantes en las escalas del Aprendizaje Colaborativo en Línea, principalmente en la Relevancia, Interactividad y Pensamiento Reflexivo.

A partir de estos resultados surgen dos recomendaciones principales: la primera, al utilizar estrategias de gamificación es necesario articular el tipo de aprendizaje que se está buscando y las mecánicas o estrategias que se emplearán para el curso; aunque existe una amplia variedad de mecánicas y estrategias que pueden emplearse y que los investigadores han documentado en sus estudios, es conveniente iniciar con aquellas que se consideran básicas y que han tenido los mayores análisis, específicamente en lo referente a los puntos, las insignias, las tablas de clasificación y las narrativas. En el caso de usar estrategias que se considerarían más complejas como el EscapeRoom, los profesores y las profesoras deben estar familiarizados con sus características y prever sus posibles efectos en el alumnado, ya que se requiere mayor preparación en el diseño de las actividades, principalmente para trabajar en la modalidad a distancia. La segunda recomendación es hacer mediciones con instrumen- tos ya validados para tener indicadores fiables y monitorear al grupo antes, durante y al final del curso a distancia. Utilizar el instrumento COLLES es confiable y válido para identificar el aprendizaje colaborativo de los y las estudiantes, permite al profesorado tomar decisiones oportunamente para mejorar el diseño de los cursos a distancia, en la experiencia de estudiantes con las actividades que realicen y mantener la motivación e interés hacia los temas y para continuar con sus estudios. De esta manera, es posible lograr mejores indicadores sobre la deserción escolar y la aceptación de la sociedad de la modalidad de educación a distancia, independientemente de que sea necesario que las actuales y futuras generaciones estudien de esta forma, ya que lo más importante es combinar la tecnología y las buenas prácticas educativas.

\subsection{Limitaciones y delimitaciones}

No hubo procedimiento muestral porque los y las estudiantes se inscribieron al curso de manera voluntaria y de acuerdo con los procedimientos fijados por la institución; tampoco se consideró el género ni el grupo escolar al que pertenecían como variables del estudio. Por otra parte, el instrumento fue administrado al final de cada unidad con el fin de identificar el nivel de aprendizaje colaborativo logrado por cada estrategia, a diferencia de otros estudios donde se administró al principio y al final de cada unidad para comparar ambos puntajes, a este procedimiento se le conoce como "encuesta preferida y real" (Rivero, 2018, p. 179; Azhari et al., 2020, p. 274; SyedMohamad et al., 2006, p. 187; Sthapornnanon et al., 2009, p. 5).

\section{Apoyos}

Este trabajo fue parte del proyecto "Estrategias de Gamificación a Distancia en la Educación Superior" que fue financiado por el Programa para el Desarrollo Profesional Docente, para el Tipo Superior (PRODEP) de la Secretaría de 
Educación Pública (SEP) en México, en el marco de la convocatoria "Apoyo a la incorporación de nuevos Profesores de Tiempo Completo (PTC)".

\section{Referencias bibliográficas}

Al-Jedaiah, M. (2020). Knowledge Management and E-Learning Effectiveness: Empirical Evidence from Jordanian Higher Education Institutions. International Journal of Emerging Technologies in Learning, 15(5), 50-62. https://doi.org/10.3991/ijet.v15i05.11653

Al-Samarraie, H., \& Saeed, N. (2018). A systematic review of cloud computing tools for collaborative learning: Opportunities and challenges to the blended-learning environment. Computers \& Education, 124, 77-91.

https://doi.org/10.1016/j.compedu.2018.05.016

Azhari, F., Jasmi, N., Abd, M., Jofrry, S., Lee, K., \& Ming, L. (2020). Students' Perceptions about Social Constructivist Learning Environment in e-learning. Indian Journal of Pharmaceutical Education and Research, 54(2), 271-278. https://doi.org/10.5530/ijper.54.2.31

Baker, J. (2005). Constructivist Online Learning Environment Survey. In R. Reynolds, Handbook of Research on Electronic Surveys and Measurements (pp. 299-301). Idea Group Inc. https://doi.org/10.4018/9781591407928.ch036

Barna, B., \& Fodor, S. (2019). Complex Gamification Platform Based on Moodle System. 16th International Conference on Cognition and Exploratory Learning in Digital Age (CELDA 2019) (pp. 409-412). IADIS.

https://doi.org/10.33965/celda2019_201911C052

Bartlet, K., \& Anderson, J. (2019). Gaming to Learn: Bringing Escape Rooms to the Classroom. In P. Pull \& J. Keengwe, Handbook of Research on Innovative Digital Practices to Engage Learners (pp. 1-24). IGI Global. http://doi:10.4018/978-1-5225-9438-3.ch001

Boverman, K., \& Bastiens, T. (2020). Towards a motivational design? Connecting gamification user types and online learning activities. Research and Practice in Technology Enhanced Learning, 15(1).

https://doi.org/10.1186/s41039-019-0121-4
Buendgens, J. (2021). Digital storytelling: multimodal meaning making. In T. Beaven \& F. Rosell (Eds.), Innovative language pedagogy report (pp. 103-108). Research-publishing.net. https://doi.org/10.14705/rpnet.2021.50.1243

Deterding, S., Sicart, M., Nacke, L., \& Dixon, D. (2011). Gamification. using game-design elements in non-gaming contexts. CHI ' 11 : $\mathrm{CHI}$ Conference on Human Factors in Computing Systems (pp. 2425-2428). ACM.

https://doi.org/10.1145/1979742.1979575

Dougiamas, M., \& Taylor, P. (2002). Interpretive analysis of an internet-based course constructed using a new courseware tool called Moodle. https://bit.ly/3leIlpM

Duggal, K., Singh, P., \& Gupta, L. (2021). Impact of Gamification, Games, and Game Elements in Education. In P. Singh, Z. Polkowski, S. Tanwar, S. Pandey, G. Matei, \& D. Pirvu, Innovations in Information and Communication Technologies (pp. 201-210). Springer.

https://doi.org/10.1007/978-3-030-66218-9_23

Fernandes, F., Mateus, A., Leonor, S., Sequeira, M., \& Gaio, R. (2018). Gamethinking: a roadmap to a Design Thinking-based model for Game Development education. Revista Lusófona de Educação (40), 167-176.

https://doi.org/10.24140/issn.1645-7250.rle40.11

Fotaris, P., Mastoras, T., Leinfellner, R., \& Rosunally, Y. (2016). Climbing Up the Leaderboard: An Empirical Study of Applying Gamification Techniques to a Computer Programming Class. The Electronic Journal of e-Learning, 14(2), 94-110. https://bit.ly/3i2HkyY

Fraternali, P., \& Herrera, S. (2019). Model Driven Development of Gamified Applications. Journal of Web Engineering, 18(7), 655-694. https://doi.org/10.13052/jwe1540-9589.1874

Gambarato, R., \& Dabagian, L. (2016). Transmedia dynamics in education: the case of Robot Heart Stories. Educational Media International, 53(4), 229-243.

https://doi.org/10.1080/09523987.2016.1254874

Gutiérrez, O., \& Duche, A. (2021). Integration model for collaborative learning in Covid-19 times: Validation of the Constructivist On-Line Learning Environment Survey (COLLES). 16th Iberian Conference on Information 
Systems and Technologies (CISTI) (pp. 1-5). IEEE.

https://doi.org/10.23919/CISTI52073.2021.9476272

Hamari, J., Shermoff, D., Rowe, E., Asbell-Clarke, J., \& Edwards, T. (2016). Challenging games help students learn: An empirical study on engagement, flow and immersion in game-based learning. Computers in Human Behavior, 54, 170179. https://doi.org/10.1016/j.chb.2015.07.045 Hancock, G. (2004). Experimental, quasi-experimental, and nonexperimental design and analysis with latent variables. In D. Kaplan, The Sage Handbook of Quantitative Methodology for the Social Sciences (pp. 317-334). Sage Publications, Inc.

Hunt, C., Moreno, O., Moreno, P., \& Ferras, M. (2020). Escape rooms' pedagogical potential from female future teachers' perspectives. Journal of University Teaching and Learning Practice, 17(5), 1-16. https://bit.ly/36ZuEm2

Jiménez, C., Arís, N., Magreñán, A., \& Orcos, L. (2020). Digital Escape Room, Using Genial.Ly and A Breakout to Learn Algebra at Secondary Education Level in Spain. Education Sciences, 10(10).

https://doi.org/10.3390/educsci10100271

Kapp, K., Blair, L., \& Mesch, R. (2014). The Gamificaction of learning and instruction. Wiley.

Khoshkangini, R., Valetto, G., Marcon, A., \& Pistore, M. (2021). Automatic generation and recommendation of personalized challenges for gamification. User Modeling and UserAdapted Interaction, 31(1), 1-34.

https://doi.org/10.1007/s11257-019-09255-2

Kingsley, T., \& Grabner-Hagen, M. (2018). Vocabulary by Gamification. The Reading Teacher, 71(5), 545-555. https://doi.org/10.1002/trtr.1645

Kraft, M. (2020). Interpreting Effect Sizes of Education Interventions. Educational Researcher, 49(4), 241-253.

https://doi.org/10.3102/0013189X20912798

Kumi-Yeboah, A. (2018). Designing a cross-cultural collaborative online learning framework for on line instructors. Onlibe Learning, 22(4), 181-201.

https://doi.org/10.24059/olj.v22i4.1520
Laal, M., \& Ghodsi, S. (2012). Benefits of collaborative learning. Procedia-Social and Behavioral Sciences, 31, 486-490.

https://doi.org/10.1016/j.sbspro.2011.12.091

Laal, M., \& Laal, M. (2012). Collaborative learning: what is it? Procedia-Social and Behavioral Sciences, 31, 491-495.

https://doi.org/10.1016/j.sbspro.2011.12.092

Laine, T., \& Lindberg, R. (2020). Designing Engaging Games for Education: A Systematic Literature Review on Game Motivators and Design Principles. IEEE Transactions on Learning Technologies, 13(4), 804-821.

https://doi.org/10.1109/TLT.2020.3018503

Lawrence, R., \& Paige, D. (2016). What Our Ancestors Knew: Teaching and Learning through Storytelling. New Directions for Adult and Continuing Education (149), 63-72.

https://doi.org/10.1002/ace.20177

Pattanshetti, M., Jasola, S., Gupta, V., \& Rajput, A. (2018). The open corpus challenge in eLearning. Knowledge Management \& E-Learning: An International Journal, 10(1), 67-85. https://doi.org/10.34105/j.kmel.2018.10.004

Pikhart, M., \& Klimova, B. (2019). Utilization of Linguistic Aspects of Bloom's Taxonomy in Blended Learning. Educ. Sci., 9(3), 235. https://doi.org/10.3390/educsci9030235

Poondej, C., \& Lerdpornkulrat, T. (2019). Gamification in e-learning: A Moodle implementation and its effect on student engagement and performance. Interactive Technology and Smart Education, 17(1), 56-66.

https://doi.org/10.1108/ITSE-06-2019-0030

Rebelo, S., \& Isaías, P. (2020). Gamification as an engagement tool in e-learning websites. Journal of Information Technology Education: Research, 19, 833-854. https://doi.org/10.28945/4653

Rivero, M. (2018). Student's Perception About Quality of a Blended Learning Environment with Moodle Assistance. Pixel-Bit. (53), 193-205. http://dx.doi.org/10.12795/pixelbit.2018. i53.13

Rodríguez, A., \& Arias, A. (2020). Revisión de propuestas metodológicas: Una taxonomía de agrupación categórica. Alteridad, 15(2), 146-160. https://doi.org/10.17163/alt.v15n2.2020.01

Sheldon, L. (2012). The multiplayer classroom. Cengage Learning. 
Silva, R., Rodrigues, R., \& Leal, C. (2019). Gamification in management education. A literature mapping. Education and Information Technologies, $25,1803-1835$.

https://doi.org/10.1007/s10639-019-10055-9

Sthapornnanon, N., Sakulbumrungsil, R., Theeraroungchaisri, A., \& Watcharadamrongkun, S. (2009). Social Constructivist Learning Environment in an Online Professional. American Journal of Pharmaceutical Education, 73(1), 1-9.

Syed-Mohamad, S., Pardi, K., Zainal, N., \& Ismail, Z. (2006). Expanding nursing education through e-learning: A case study in Malaysia. In H. Park, P. Murray, \& C. Delaney, Studies in Health Technology and Informatics (Vol. 122, pp. 186-189). IMIA. https://bit.ly/3ydP7Qc

Taylor, P., \& Maor, D. (2000). Assessing the efficacy of online teaching with the Constructivist On-Line Learning Environment Survey. In H. A., \& M. Kulski, Flexible Futures in Tertiary Teaching. Proceedings of the 9th Annual Teaching Learning Forum.

https://bit.ly/31LAfgZ

Teo, T., Kim, S., \& Jiang, L. (2020). E-Learning Implementation in South Korea: Integrating Effectiveness and Legitimacy Perspectives. Information Systems Frontiers, 22(2), 511-528. https://doi.org/10.1007/s10796-018-9874-3
Uz, C., \& Gul, A. (2020). Investigating the Effectiveness of Gamification on Group Cohesion, Attitude, and Academic Achievement in Collaborative Learning Environments. TechTrends, 64, 124136.

https://doi.org/10.1007/s11528-019-00442-x

Werbach, K., \& Hunter, D. (2015). Gamification Toolkit: Dynamics, Mechanics, and Components for the Win. Wharton Digital Press.

Wiggins, B. (2016). An Overview and Study on the Use of Games, Simulations, and Gamification in Higher Education. International Journal of Game-Based Learning (IJGBL), 6(1), 18-27. https://doi.org/10.4018/IJGBL.2016010102

Yen, S., Lo, Y., Lee, A., \& Enriquez, J. (2018). Learning online, offline, and in-between: comparing student academic outcomes and course satisfaction in face-to-face, online, and blended teaching modalities. Education and Information Technologies, 23, 2141-2153. https://doi.org/10.1007/s10639-018-9707-5

Yeo,S., Taylor, P., \& Kulski,M.(2006).Internationalising a learning environment instrument for evaluating transnational online university courses. Learning Environments Research, 9(2), 179-194.

https://doi.org/10.1007/s10984-006-9008-6 


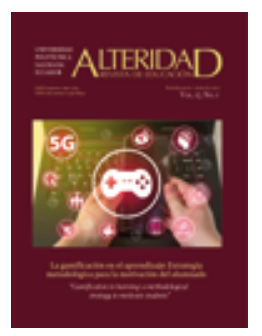

\title{
¿Jugamos o gamificamos? Evaluación de una experiencia formativa sobre gamificación para la mejora de las competencias digitales del profesorado universitario
}

\author{
Do we play or gamify? Evaluation of gamification training \\ experience to improve the digital competence of university \\ teaching staff
}

Lorena Martín-Párraga predoctoral, Universidad de Sevilla (España) (lorena@grupotecnologiaeducativa.es) (https://orcid. org/0000-0002-2406-0708)

D Antonio Palacios-Rodríguez contrato predoctoral, Universidad de Sevilla (España) (aprodriguez@us.es) (https://orcid. org/0000-0002-0689-6317)

(D) Dr. Óscar Manuel Gallego-Pérez es profesor e investigador de la Universidad de Sevilla (España) (ogallego@us.es) (https:// orcid.org/0000-0001-8450-8634)

Recibido: 2021-09-09 / Revisado:: 2021-11-07 / Aceptado: 2021-11-26 / Publicado: 2022-01-01

\section{Resumen}

En las últimas décadas se han realizado estudios en el ámbito de lo educativo que avalan los beneficios que la gamificación ofrece tanto para docentes como al alumnado. En esta línea se trabaja desde la universidad, con la finalidad de conseguir desarrollar habilidades óptimas que beneficien al alumnado, tanto personal como profesionalmente. En consecuencia, esta formación diseñada y seleccionada para docentes es esencial para conseguir una experiencia gamificada exitosa. El objetivo principal de esta investigación es exponer una experiencia formativa llevada a cabo en una universidad de América Latina y comprobar la percepción de esta estrategia metodológica por parte de la institución educativa. Es decir, la finalidad consistiría en demostrar si la gamificación es percibida como útil por parte del profesorado universitario. Para ello, se han empleado dos diseños: descriptivo y la validación mediante ecuaciones estructurales (PLS), de forma que se pudiese evaluar el grado de aceptación que tiene la gamificación como metodología activa en base con el modelo de Aceptación de la Tecnología (TAM). Los resultados expuestos demuestran que la propuesta es adecuada para la docencia universitaria. Todos los participantes, I | 4, percibieron esta estrategia como adecuada, detallando las dimensiones relacionadas con la facilidad de uso en el aula, su integración, la transformación del interés en el aprendizaje, y la capacidad de mostrar una actitud positiva en su uso. Simultáneamente, el nivel de aceptación respecto a la validación del TAM es alto. Por último, detallar la valoración a la hora de replicar el modelo en investigaciones futuras y similares, o a través de las llamadas tecnologías emergentes.

Descriptores: Gamificación, Educación Superior, competencia digital, formación del profesorado, metodologías activas, validación.

\begin{abstract}
Over the last few decades, studies have been carried out in the field of education that endorse the benefits that gamification offers both for teachers and students. The university is working along these lines, with the aim of developing optimal skills that benefit students, both personally and professionally. Consequently, this training designed and selected for teachers is essential to achieve a successful gamified experience. The main purpose of this research is to present a training experience carried out in a university in Latin America and to verify the perception of this methodological strategy by this educational institution. In other words, the aim is to demonstrate whether gamification is perceived as useful by university teaching staff. To this end, two designs were used: descriptive and validation by means of structural equations (PLS), in order to evaluate the degree of acceptance of gamification as an active methodology based on the Technology Acceptance
\end{abstract}

Forma sugerida de citar: Martín-Párraga L., Palacios-Rodríguez, A., \& Gallego-Pérez, Ó. M. (2022). ¿Jugamos o gamificamos? Evaluación de una experiencia formativa sobre gamificación para la mejora de las competencias digitales del profesorado universitario. Alteridad, (17)1, 36-49. https://doi.org/10.17163/alt.v17n1.2022.03 
Model (TAM). The results show that the proposal is suitable for university teaching. All the participants, I14, perceived this strategy as adequate, detailing the dimensions related to the ease of use in the classroom, its integration, the transformation of interest in learning, and the ability to show a positive attitude in its use. At the same time, the level of acceptance regarding the validation of the TAM is high. Finally, the assessment of the replicability of the model in future and similar research, or through so-called emerging technologies, is detailed.

Keywords: Gamification, Higher education, digital competence, teacher training, active methodologies, validation.

\section{Introducción}

Actualmente, la sociedad del siglo XXI se encuentra sumergida en constantes retos tecnológicos y metodológicos, viéndose obligada a transformar su práctica educativa. La adecuación de los ambientes educativos, acorde a dichas tendencias, necesidades demandadas por el sistema y los cambios vertiginosos acaecidos, influyen en la forma de comunicarnos, aprender e interactuar en la llamada sociedad de la información y la comunicación. Una sociedad hipertecnologizada, sumergida en continuos y múltiples cambios, muchos de ellos producidos por el uso e implementación de las tecnologías digitales (Tornero \& Varis, 2010). Nuevos medios comunicacionales que alfabetizan las prácticas, sumándole importancia a la hora de interpretar el funcionamiento de los contextos laborales y recreativos de la comunidad del conocimiento del siglo XXI (Mills, 2010)

Una nueva era tecnológica, fruto de los cambios acelerados, donde la información abre caminos inescrutables promovidos por los avances caracterizados por el uso de las llamadas Tecnologías de la Información y la Comunicación (TIC). La posibilidad de acceder, de manera inmediata, a cada uno de los sectores que componen dicho ciclo anuncia los ingredientes esenciales generadores de un gran valor añadido al desarrollo económico y social de la catalogada sociedad del conocimiento.

El tecnologizar la información es el fin que impulsa y genera nuevas alteraciones en la organización del conocimiento, las prácticas y las formas de organización, así como en el moldeamiento de la cognición humana, sin obviar, y con ello, la temática que nos concierne; el ámbito de lo educativo.

Todo ello pone de manifiesto la necesidad de la transformación digital docente y la adquisición de competencias, definidas, según la Unión Europea, como "una combinación de conocimientos, capacidades y actitudes adecuadas al contexto" (Consejo de la Unión Europea, 2018 , p.7). Se hace por tanto imprescindible la adquisición de competencias que respondan a los desafíos actuales.

Pese a esta progresión, el mero hecho de incorporar las tecnologías no hace que cambien los ambientes de aprendizajes. Según Pelgrum y Voogt (2009), para conseguir dicho propósito, debe existir un liderazgo en los centros que consiga aumentar el deseo, por parte de docentes, a la hora de utilizar nuevos métodos de enseñanza en relación con las TIC, para así poder adquirir mayores niveles competenciales en el uso de las TIC y generar el desarrollo de una cultura colaborativa que fomente la inclusión de las TIC en el proceso de enseñanza y aprendizaje (E-A).

Es aquí donde cobra importancia el término establecido por la Comisión Europea denominado "E-Learning". La educación virtual pasaría a constituirse como una de las herramientas más poderosas cuya calidad debe medirse a la hora de diseñar planes formativos en lo relativo a dicha modalidad.

Según se detalla en la declaración de Estambul (2002) y la de Alejandría sobre Alfabetización Informacional (2005), la formación en competencias digitales es imprescindible para que un ciudadano pueda desenvolverse adecuadamente en la Sociedad del Conocimiento (Gutiérrez-Priego \& García-Peralta, 2016).

Se diría, por tanto, que la educación online es una herramienta crucial para lograr la familiarización en los contextos tecnológicos, logrando el aumento de competencias claves que permitan el desarrollo y la autonomía de buenas prácticas educativas a través de los entornos virtuales. 
Hablaríamos de un tipo de pedagogía virtual que logrará fomentar el trabajo colaborativo, mejorar la tutorización individualizada y que, además, será capaz de incluir diversidad en el aprendizaje, mejorando el nivel competencial del estudiante y el compromiso discente.

Para ello, el docente debe ser capaz de desarrollar metodologías dinamizadoras y cooperativas, que se centren en el "aprender haciendo", como método infalible hacia la mejora del rendimiento, aplicabilidad y motivación del aprendizaje estudiantil.

En definitiva, la necesidad de conseguir un modelo de alfabetización que ofrezca una cultura digital, que promueva: "la alfabetización digital, e-aprendizaje, e-inclusión, e-salud y soluciones digitales en estos campos" (Disposición 8301 de relaciones con las cortes e igualdad, 2019). A su vez, la importancia de facilitar a las comunidades educativas diferentes plataformas digitales y recursos tecnológicos y didácticos que garanticen su correcta aplicación en el proceso de E-A (Disposición 8301 de relaciones con las cortes e igualdad, 2019).

La importancia de hacer uso de las TIC para facilitarnos las labores diarias, engrandecernos profesionalmente y como formación a lo largo de la vida es un aspecto relevante y sustancial cara desempeñar cualquier programa formativo (Cabero-Almenara \& Palacios-Rodríguez, 2020). El considerable aumento de dicha progresión supondrá que en un futuro próximo los puestos de trabajo demandarán habilidades digitales (Williamson et al., 2019).

Cuando hablamos de incluir un modelo competencial en un currículum, nos referimos a que las actividades de aprendizaje no deben limitarse a una sola materia, sino que los contenidos, desarrollados en ella, busquen alcanzar una misma competencia que les permita afrontar cualquier situación. Debemos ser capaces de conseguir un cambio de metodologías, logrando pasar de un tipo de metodología reproductiva a una productiva. Únicamente de esta forma conseguiremos que el alumnado sea capaz de transportar el conocimiento adquirido en una materia de resolución de problemas en diferentes escenarios. La consolidación de las competencias mediante estrategias productivas potencia, a la vez, distintos aspectos del proceso de enseñanzaaprendizaje (E-A).

En este sentido, con la finalidad principal de ofrecer soluciones a dichas alteridades, se plantean nuevas formas de interpretar los entornos educativos, buscando una transformación que consiga una mejor adaptación didáctica en dicho sector. Estas metodologías activas hacen que el y la estudiante adquieran un rol dinámico en su propio aprendizaje, logrando transformar concepciones rígidas y memorísticas.

En la mayoría de nuestras universidades se continúan utilizando metodologías de aprendizaje tradicionales centradas en el profesor y no en el estudiante (Lai et al., 2018; Pelger \& Nilsson, 2018). En consecuencia, encaminados en la búsqueda de una mejor adaptación a los contextos actuales, abordar la diversidad e igualdad de condiciones en las aulas y desarrollar diferentes formas de motivar y dinamizar el proceso de E-A surge la gamificación, también denominada ludificación. Esta estrategia consiste en introducir actividades en el aula a través de las dinámicas propias de los juegos, permitiendo la construcción de aprendizajes dinámicos donde se potencie el aumento de la participación del estudiante y, por consiguiente, que este adquiera la necesidad de "aprender". Es decir, la gamificación es un tipo de estrategia que consigue fomentar un aprendizaje más significativo enfocado en los intereses del alumnado (Kapp, 2012; Herberth-Alexander, 2016; Molina-Álvarez et al., 2017; Corchuelo-Rodríguez, 2018). Esta forma de replantear la educación trae consigo nuevas metodologías y, por ende, la necesidad de una transformación digital y la adquisición de competencias claves que logren ofrecer una educación de calidad mediada en los entornos de transformación digital actuales.

Son diversos los campos de aplicación que ofrece la gamificación, siendo el más investigado 
el del ámbito de lo educativo (Domínguez-Díaz, 2018), donde surge el implementarla en los entornos de e-learning, dada su naturaleza digital (Muntean, 2011). Esto conlleva, que, en los últimos tiempos, estas prácticas gamificadoras estén adquiriendo un protagonismo relevante (Peñalva et al., 2018). Sobre todo, por la diversidad de posibilidades que ofrecen tal y como están poniendo de manifiesto los diversos metaanálisis sobre la temática (Martínez-García et al., 2020). Un estudio llevado a cabo por Çakıroğlu et al. (2017), donde se presentaron pruebas de índole real, reveló el efecto positivo que la gamificación integra en la educación y como esta logra influir, indirectamente en el rendimiento académico, el compromiso personal y las relaciones sociales, generando efectos positivos e impactos motivacionales que benefician el proceso de E-A.

Este tipo de aprendizaje se convierte en óptimo, debido al disfrute en su realización y con ello, una mejor adquisición de los contenidos (Molina-Álvarez et al., 2017). Se entiende que, en los juegos, los retos plantean la necesidad que posee el jugador a la hora de superar sus expectativas, conllevando esto una gran carga psicológica, con el objetivo de influir en el comportamiento humano (De Soto García, 2018).

Muchos de los estudios realizados en el ámbito estudiado llegan a la conclusión de que la gamificación puede ofrecer diferentes ventajas tanto para profesores como para estudiantes. $\mathrm{Al}$ profesor, por ejemplo, le permite comprender mejor los estilos y dificultades de aprendizaje de los estudiantes y ofrecer un feedback más inmediato, así como aprovechar el tiempo de clase para la comprensión de los contenidos (Ortiz-Colón et al., 2018). Por otro lado, los y las estudiantes se involucran más que en las clases tradicionales, provocando emociones y actitudes positivas hacia el aprendizaje (Gallego-Durán et al., 2014).

La gamificación ha demostrado ser eficaz en términos de adquirir conocimientos y habili- dades para el futuro profesional de los estudiantes (Villalustre-Martínez \& del Moral, 2015). A su vez, otros autores afirman que podría utilizarse como mecanismo motivacional que fomente entre alumnos comportamientos positivos para su formación (Kapp, 2012; Smith-Robbins, 2011). Centrándose en la relación entre el aprendizaje basado en actividades gamificadas, Donoso-Anés y López-Gavira (2010) observan que se aumentan las oportunidades de instrucción entre compañeros cuando las actividades en clase promueven el aprendizaje activo. En definitiva, la gamificación es una metodología activa la cual surge por el interés docente a la hora de mejorar y motivar el compromiso del estudiante en su autoaprendizaje (Rodríguez-Jiménez et al., 2019).

Finalmente señalar que, aunque muchos estudios han investigado sobre las actividades y prácticas específicas que se utilizan la gamificación (Cortizo-Pérez et al., 2011), no se ha prestado la suficiente atención al resultado final de las mismas, ni a la satisfacción de los estudiantes ni profesorado que la lleva a cabo.

Para analizar el grado de aceptación de la metodología gamificadora en este estudio, se usa uno de los modelos que mayor significación ha adquirido para explicar el grado de adopción que una persona hace de una tecnología, tanto general como concreta. Este modelo se denominó originalmente "Technology Acceptance Model" (TAM), de Davis (1989). Bajo su paraguas se recoge que las creencias, actitudes o predisposiciones que se tengan sobre las tecnologías repercutirán sobre el empleo que se realice de él. Para ello, está determinadas por dos variables: la utilidad percibida y la facilidad de uso percibida. El modelo TAM propone y demuestra empíricamente que la Percepción de Utilidad (PU) y la Percepción de la Facilidad de Uso (PFU) son los factores más críticos en el proceso de adopción de la tecnología y el uso de los sistemas (Chen \& Tan, 2004). 


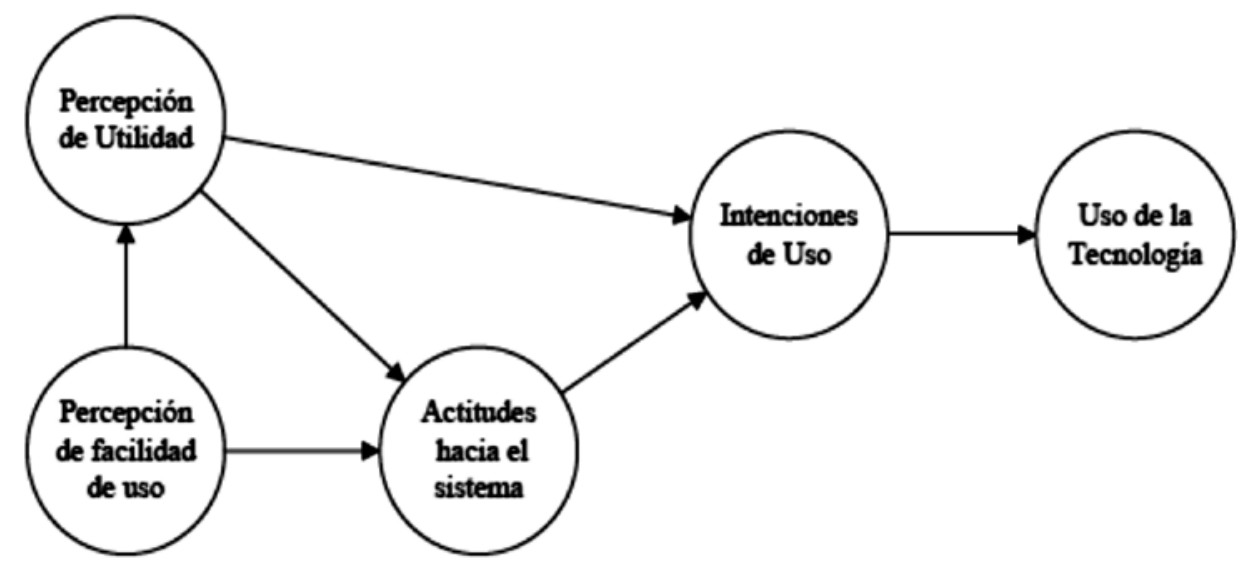

En diferentes estudios se han incorporado diferentes transformaciones (López-Bonilla \& López-Bonilla, 2017; Cabero-Almenara et al., 2016; Cabero-Almenara \& Llorente, 2020; CaberoAlmenara et al., 2021b). Esto se ha realizado a través de la incorporación de nuevas dimensiones como, por ejemplo, dimensiones predictoras (género, grado de obligatoriedad de adoptar la tecnología, experiencia, edad, tipo de usuarios...).

Desde el propio modelo se hace hincapié en que este debe ser construido en cada y para cada situación de aceptación de las tecnologías que se deseen investigar. Por este motivo, se adapta de manera excepcional al estudio que se presenta, contextualizando y ubicando en él las variables mediadoras que se consideran desde el punto de vista del investigador. En este sentido, el modelo ha evolucionado hacia otras versiones, tales como el TAM 2 (Venkatesh \& Davis, 2000) o el modelo de integración de la aceptación de la tecnología y la satisfacción del usuario (Venkatesh \& Bala, 2008).

\section{Metodología}

Con base en las líneas anteriores, esta investigación presenta el diseño de una propuesta formativa destinada a docentes latinoamericanos de Educación Superior. En este sentido, se desarrolla el proceso formativo y se evalúa el grado de aceptación de esta metodología por parte del profesorado. Por ende, el objetivo propuesto es estudiar el nivel de aceptación de la metodología gamificadora del profesorado universitario implicado en la acción formativa: validación del modelo de aceptación de la metodología gamificadora (TAM) y análisis del nivel de aceptación de la metodología gamificadora.

Este estudio utiliza dos tipos de diseños: validación de escala y análisis descriptivo. El primero de ellos trata de validar la escala de aceptación de la metodología gamificadora adaptando el modelo TAM de Davis (1989). Posteriormente, se procede a aportar los datos más significativos relacionados con la aceptación de la metodología gamificadora.

Los 114 participantes son profesores (54) y profesoras (60) de la Universidad Técnica Particular de Loja (UTPL), en Ecuador. Dicho profesorado forma parte de la plantilla de la universidad encargada de desarrollar clases en la modalidad presencial y virtual de los diferentes grados y posgrados que oferta.

La UTPL es una institución autónoma, con finalidad social y pública, imparte enseñanza, desarrolla investigaciones con libertad científica-administrativa, y participa en planes de desarrollo del país.

Se presenta la experiencia llevada a cabo con el profesorado de la UTPL. Las finalidades y 
objetivos que se persiguen con la acción formativa son las siguientes:

- Conocer conceptos básicos relacionados con el juego, los videojuegos, el Aprendizaje Basado en Juegos (ABJ) y la gamificación.

- Gamificación en Educación Superior.

- Conocer la importancia de la aplicación del término en los escenarios formativos.

- Conocer lo diferentes elementos del juego que resultan relevantes para su aplicación en los ámbitos que se demanden.

- Ver diversos ejemplos de aplicaciones educativas gamificadoras.

- La adquisición de estrategias que posibiliten la aplicación de dicha estrategia en la docencia.

- Desarrollar competencias para la producción de recursos gamificadores mediante software libre o con licencia educativa.

- Producir recursos gamificadores para una materia.

Los contenidos que se desarrollaran en el curso son los siguientes:

- Gamificación.

- Teorías que fundamentan la gamificación: Teoría de Flujo y Modelo de Fogg.

- Técnicas y dinámicas de la gamificación.

- Gamificación en el proceso de E-A.

- Gamificación en la Enseñanza Superior.

- Aprendizaje Basado en Juegos (ABJ)

- El Juego en la enseñanza universitaria

- Herramientas para gamificar

1. Edmodo

2. Class Dojo

3. Quizizz

4. Mentimeter

5. Socrative

6. Kahoot

7. Studio

Integración de herramientas gamificadoras en Canvas
Los tres formadores encargados de la misma pertenecen a la Universidad de Sevilla y realizan dicha acción de forma virtual. Los participantes disponen en el aula de todos los contenidos y recursos necesarios, que se completan con tres sesiones de videoconferencia en las que se tratan aspectos teóricos y prácticos sobre la temática.

Los materiales del curso están formados por diferentes tipos, fundamentalmente por fragmentos de texto, esquemas, gráficos y clip de vídeos. Se presentan una serie de enlaces que van dirigidos a diferentes documentos, dichos documentos, y se indican en el texto, son para saber más, y no son de obligada lectura para el seguimiento de la acción formativa.

En cuanto a las tutorías, los tutores tienen presencia diaria en el curso, dinamizando la participación y animando a resolver las dudas colaborativamente o contestándolas directamente. La resolución de dudas tiene lugar a través de un foro de dudas que se abre en la plataforma virtual a tal efecto.

La evaluación final del curso se realizará con base en tres ejes:

- Realización de las actividades que en la formación a distancia se establezcan.

- Participación en las sesiones por videoconferencia.

- Realización de actividad final (producción material gamificador para una de las asignaturas).

Para poder medir el grado de motivación, así como también el nivel de satisfacción experimentado por el profesorado universitario a la hora de participar en experiencias formativas apoyadas en la gamificación y poder indagar sobre las dificultades técnicas, curriculares y organizativas que se presenten, se administra el modelo TAM (Modelo de Aceptación de la Tecnología, de Davies,1989). Su aplicación se realiza de manera telemática a través de la siguiente dirección: https://cutt.ly/8WHa4FS 
Previo al análisis de los datos, se comprueba que estos no se distribuyen normalmente a través del estudio de asimetría y curtosis. La prueba de "bondad de ajuste KolmogorovSmirnov" confirma esta comprobación, con significación (p-valor) igual a .000 para todos los ítems (distribución no normal).

Para alcanzar el objetivo principal, se procede a validar el modelo de aceptación de la metodología gamificadora (TAM-GAM) mediante un análisis de la fiabilidad (Alfa de Cronbach y fiabilidad compuesta), validez de constructo (AVE) y modelado de ecuaciones estructurales (PLS). Tras ello, se realizan análisis descriptivos y de tendencia central.

Los datos obtenidos son analizados con el paquete estadístico SPSS 27 (análisis descriptivo y contraste), y software SmartPLS 3 (modelado ecuaciones estructurales).

\section{Resultados}

Tras haber presentado la propuesta formativa, se procede a estudiar el nivel de aceptación de la metodología gamificadora. En este caso, se crea una adaptación del modelo de aceptación de la tecnología (TAM) sugerido por Davis (1988) para la metodología gamificadora. Por este motivo, antes de describir los datos, se procede a validar la adaptación realizada.

Primero, se arrojan los valores de fiabilidad y validez de constructo. En el caso de la validez, el resultado global tras aplicar la Alfa de Cronbach es de 0.912. Según O’Dwyer y Bernauer (2013), esta cifra implica un alto nivel de fiabilidad. Conjuntamente, se procede a calcular los índices de fiabilidad, fiabilidad compuesta y validez de constructo por dimensiones. Los resultados son mostrados en la Tabla 1.

Tabla 1. Valores de fiabilidad y validez de constructo por dimensiones

Alfa de Cronbach

\section{Fiabilidad compuesta}

\begin{tabular}{|c|c|c|c|}
\hline UP & 0.914 & 0.956 & 0.824 \\
\hline FUP & 0.802 & 0.872 & 0.821 \\
\hline PD & 0.926 & 0.942 & 0.865 \\
\hline ACU & 0.821 & 0.926 & 0.815 \\
\hline IU & 0.895 & 0.985 & 0.846 \\
\hline
\end{tabular}

Como se puede comprobar, todos los resultados relativos la fiabilidad (Alfa y fiabilidad compuesta) son superiores a 0.7 , valor mínimo adecuado según Lévy (2006) para indicar niveles aceptables. Por tanto, se puede afirmar que el modelo propuesto de aceptación de la metodología gamificadora presenta una buena consistencia interna en cuanto a su bloque de indicadores. En cuanto a la validez convergente, todos los índices de varianza media extraída (AVE) son superiores a 0.5 . Este valor es tomado como referencia por Bagozzi y Yi (1988) para indicar que más del $50 \%$ de las varianzas de constructo son debidas a los indicadores del modelo. Por ende, se puede afirmar que la cantidad total de la varianza de los indicadores es tenida en cuenta por el constructo latente.

Tras ello, se analiza la validez discriminante del modelo que permite conocer si cada dimensión es diferente a las otras. Para ello, se emplean dos técnicas: criterio de Fornell-Larcker y análisis de las cargas cruzadas.

El criterio de Fornell-Larcker permite comprobar si la varianza extraída media de una dimensión es mayor que la varianza del resto de dimensiones. En la Tabla 2 se muestran los resultados. 
Tabla 2. Validez discriminante del modelo mediante el criterio de Fornell-Larcker

\begin{tabular}{|c|c|c|c|c|c|}
\hline & ACU & FU & IU & PD & UP \\
\hline$A C U$ & 0.912 & & & & \\
\hline FU & 0.321 & 0.852 & & & \\
\hline IU & 0.782 & 0.280 & 0.941 & & \\
\hline PD & 0.781 & 0.291 & 0.663 & 0.960 & \\
\hline UP & 0.715 & 0.405 & 0.700 & 0.600 & 0.900 \\
\hline
\end{tabular}

Para interpretar esta tabla, se debe tener en cuenta que los elementos de la diagonal son la raíz cuadrada de la varianza extraída media, y los otros las correlaciones entre dimensiones. Como se puede comprobar, todos los valores que están a la izquierda y por debajo de los valores de la diagonal son menores a ellos. Por tanto, se confirma el primer criterio de validez discriminante. A continuación, se realiza el análisis de las cargas cruzadas del modelo. Los resultados pueden observarse en la Tabla 3.

Tabla 3. Cargar cruzadas del modelo

\begin{tabular}{|c|c|c|c|c|c|}
\hline & UP & FUP & PD & ACU & IU \\
\hline UP1 & 0.902 & & & & \\
\hline UP2 & 0.802 & & & & \\
\hline UP3 & 0.905 & & & & \\
\hline UP4 & 0.901 & & & & \\
\hline FUP1 & & 0.812 & & & \\
\hline FUP2 & & 0.800 & & & \\
\hline FUP3 & & 0.910 & & & \\
\hline PD1 & & & 0.963 & & \\
\hline PD2 & & & 0.921 & & \\
\hline PD3 & & & 0.854 & & \\
\hline ACU1 & & & & 0.903 & \\
\hline ACU2 & & & & 0.910 & \\
\hline IU1 & & & & & 0.910 \\
\hline IU2 & & & & & 0.952 \\
\hline
\end{tabular}

Los resultados, superiores a 0.7 , indican altos niveles de correlación (O'Dwyer y Bernauer, 2013). Por tanto, se asegura que los ítems miden el constructo al que se han incorporado. Tras ello, se procede a presentar el modelo formulado mediante la obtención de los coeficientes de regresión estandarizados (path coefficients), los valores de la t de student y los R2 (R-cuadrado) del diagrama estructural. En lo que se refiere a los resultados, el modelo explica un $73 \%$ de la varianza en la dimensión "actitud hacia el uso", un $62 \%$ en la dimensión "intención de uso", un $40 \%$ en la dimensión "percepción de disfrute" y un $23 \%$ en la dimensión "utilidad percibida". Todas las relaciones del modelo son significativas con un nivel de confianza del $99 \%$. 
Finalmente, se evalúa la bondad de ajuste del modelo mediante la media cuadrática estandarizada (SRMR), Chi-cuadrado e índice de ajuste normalizado (NFI). En la Tabla 4 se representan los valores obtenidos, así como los valores de referencia según Hu y Bentler (1999).

Tabla 4. Ajuste del modelo

\begin{tabular}{|l|l|l|}
\hline \multicolumn{1}{|c|}{ Indicador } & \multicolumn{1}{c|}{ Resultado } & \multicolumn{1}{c|}{ Referencia } \\
\hline SRMR & 0.063 & $<0,08$ \\
\hline Chi-cuadrado & 225.324 & $<500$ \\
\hline NFI & 0.785 & $>0.7$ \\
\hline
\end{tabular}

Tras la validación del modelo propuesto, se procede a realizar un análisis del nivel de aceptación de la metodología gamificadora. La Tabla 5 muestra las medias y desviaciones típicas de cada uno de los ítems que conforman el modelo. Para su interpretación, se debe tener en cuenta que la escala usada tiene 7 puntos $(1=$ Totalmente en desacuerdo $/ 7=$ Totalmente de acuerdo).

Tabla 5. Estadísticos descriptivos del nivel de aceptación de la metodología gamificadora

\begin{tabular}{|l|l|l|}
\hline \multicolumn{1}{|c|}{ Ítem } & Media & DT \\
\hline El uso de esta metodología creo que podría mejorar mi aprendizaje en el aula (UP1). & 6,43 & 0,903 \\
\hline $\begin{array}{l}\text { El uso de esta metodología durante las clases me facilitaría la comprensión de ciertos conceptos } \\
\text { (UP2). }\end{array}$ & 6,50 & 0,679 \\
\hline Creo que esta metodología es útil cuando se está aprendiendo (UP3). & 6,55 & 0,597 \\
\hline El uso de esta metodología favorece mi aprendizaje (UP4). & 6,30 & 0,758 \\
\hline Creo que la metodología es fácil de usar (FUP1). & 5,95 & 0,815 \\
\hline Aprender a usarla y manejarme con ella no ha sido un problema para mí (FUP2). & 5,35 \\
\hline Aprender a usarla y manejarme con esta metodología me ha sido clara y comprensible (FUP3). & 6,05 & 0,959 \\
\hline Utilizarla me ha sido divertido (PD1). & 6,38 & 0,705 \\
\hline Disfruté con el uso de esta metodología (PD2). & 6,35 & 0,802 \\
\hline Creo que la metodología permite aprender jugando (PD3). & 6,38 & 0,774 \\
\hline El uso de esta metodología hace que el aprendizaje sea más interesante (ACU1). & 0,679 \\
\hline Creo que su uso en el aula es buena idea (ACU2). & 6,48 \\
\hline Me gustaría utilizar en el futuro esta metodología si tuviera la oportunidad (IU1). & 5,60 & 2,521 \\
\hline $\begin{array}{l}\text { Me gustaría utilizar esta metodología para aprender tanto los temas que se me han presentado } \\
\text { como con otros temas (IU2). }\end{array}$ & 6,58 & 0,50 \\
\hline
\end{tabular}

Todos los ítems se sitúan por encima de los 5.6 puntos. Esto implica que, en general, el nivel de aceptación de la metodología gamificadora es alto. En concreto, se destaca: utilidad mientras se está aprendiendo (UP3), claridad de aprendizaje (FUP3), uso divertido y aprendizaje lúdico (PD1, PD3), interés por el aprendizaje (ACU1) e intención futura de uso para investigar otros temas (IU2).

Para concretar el análisis, se realiza un análisis descriptivo por dimensiones. Los resultados pueden ser observados en la Tabla 6 . 
Tabla 6. Estadísticos descriptivos del nivel de aceptación de la metodología gamificadora (dimensiones y total)

\begin{tabular}{|l|c|c|}
\hline \multicolumn{1}{|c|}{ Dimensión } & Media & DT \\
\hline Utilidad percibida (UP) & 6.44 & 0.73 \\
\hline Facilidad de uso percibida (FUP) & 5.95 & 1.04 \\
\hline Percepción de disfrute (PD) & 6.37 & 0.76 \\
\hline Actitud hacia el uso (ACU) & 5.21 & 1.60 \\
\hline Intención de uso (IU) & 6.54 & 0.62 \\
\hline Total & 6.16 & 0.91 \\
\hline
\end{tabular}

Tal y como se puede observar, el alumnado destaca la intención de uso (IU) y utilidad percibida (UP).

\section{Discusión y conclusión}

La metodología gamificadora es una estrategia que se está presentando como una estrategia útil y con una gran variedad de posibilidades para facilitar el aprendizaje de los y las estudiantes en diferentes áreas curriculares y en diferentes niveles educativos, desde infantil hasta universidad (Juan-Lázaro \& Area-Moreira, 2021). Sin embargo, se debe tener en cuenta que se están efectuando notables esfuerzos por realizar estudios e investigaciones con el objetivo de conocer en profundidad cómo aprenden los alumnos a través de esta.

En esta línea se ha llevado a cabo este trabajo, el cual determina como modelo significativo, dentro de la literatura científica, el TAM, formulado por Davis (1989). Este modelo propone que la utilización percibida hacia las tecnologías y el fácil manejo de estas podrán establecer valores con respecto a la actitud que se posea de la misma por parte del sujeto, siendo capaz este de dirigirla hacia una intención de uso específico. En una experiencia virtual de aprendizaje, los resultados llevan a establecer una de las primeras conclusiones; los y las docentes perciben la incorporación de la metodología gamificadora como una estrategia adecuada, destacando sobre otras dimensiones de la misma: la facilidad de uso en el aula, la aceptación de su integración, la transformación del interés por el aprendizaje, y el mostrar una actitud positiva hacia su uso en el futuro. Resultados similares a los de la investigación de Turpo-Gebera et al. (2021), donde se demuestra que la valoración satisfactoria con la formación recibida en línea indica no solo una adaptación pertinente de los recursos a sus necesidades, sino también la adquisición de mayores niveles de madurez en el dominio de competencias digitales. Con todo ello, se puede afirmar que la formación recibida por el profesorado que ha participado de esta experiencia de aprendizaje se ha desarrollado de manera satisfactoria.

Por otro lado, otra de las conclusiones que se pueden derivar de este estudio es que el nivel de aceptación en lo que a la validación del modelo TAM se refiere es alto (Teo et al., 2009; Venkatesh \& Bala, 2008). Dicho modelo presenta una adecuada consistencia interna en lo que respecta al bloque de indicadores. Por lo tanto, el modelo TAM que se emplea se presenta como un buen predictor para explicar la actitud hacia la metodología gamificadora en la educación universitaria. Todo ello pone de manifiesto la significación del modelo inicialmente formulado por Davis (1989). En este sentido, los resultados obtenidos se encuentran muy en la misma línea de los alcanzados por Arteaga y Duarte (2010), o Cabero et al. (2018).

La gamificación es una metodología activa que se presenta como útil y garante de posibilidades a la hora de facilitar y mejorar el aprendi- 
zaje de los y las estudiantes, sin importar la etapa educativa (Rodríguez-García \& Arias-Gago, 2020). Sin embargo, se debe de tener en cuenta que se están realizando considerables esfuerzos a la hora de realizar estudios e investigaciones que avalen su eficacia y con ello, conocer en profundidad el alcance que posee.

A lo largo del estudio planteado se ha profundizado en la importancia de medir el grado de aceptación de esta tecnología, por parte del profesorado, para poder determinar su utilidad futura. A pesar de presentar una simplicidad y validez alta, el modelo TAM muestra algunas limitaciones. En primer lugar, este modelo hace referencia en la predicción del uso, pero no en el incremento del rendimiento del usuario. Es decir, no existe una relación positiva entre el uso y su rendimiento. Por otro lado, encontramos la capacidad para predecir el uso real de la tecnología, ya que este instrumento se encuentra basado en el auto-reporte. Y en último lugar, la variabilidad de resultados si la muestra se aplica sobre docentes con altos niveles competenciales.

Se puede decir que el modelo analizado supone una transformación de los métodos tradicionales. Es necesario repensar las formas en las cuales se plantea el desarrollo competencial del profesorado universitario. Todo esto a través de distintos niveles y, por tanto, como un aprendizaje constante que moviliza diferentes dimensiones competenciales que van desde el dominio técnico a la innovación con TIC (Flores-Lueg \& Roig, 2016; He \& Zhu, 2017). Aun así, también es necesario apuntar distintas limitaciones del estudio como el número de muestra reducida o la especificidad del contexto donde se desarrolla la propuesta formativa. Por ello, sería interesante la replicación del modelo en estudios e investigaciones con la misma o diferente metodología como, por ejemplo, el Scape-Room o la Flipped Clasrroom, puesto que también poseen un alto potencial educativo (Cabero-Almenara et al., 2021a, 2021b), o a través de la incorporación de diferentes tecnologías, sobre todo, a través de las denominadas tecnologías emergentes (GuillénGámez et al., 2021; López-Cortes et al., 2021).

\section{Financiación}

Diseño, producción y evaluación de t-MOOC para la adquisición de competencias digitales del profesorado universitario. Proyectos $\mathrm{I}+\mathrm{D}+\mathrm{i}$ FEDER Andalucía 2014-2020. Referencia: US-1260616. Junta de Andalucía (Consejería de Economía y Conocimiento).

\section{Referencias bibliográficas}

Arteaga, R., \& Hueros, A. D. (2010). Motivational factors that influence the acceptance of Moodle using TAM. Computers in Human Behavior, 26(6), 1632-1640.

Bagozzi, R., \& Yi, Y. (1988). On the evaluationof structural equation models. Journal of the Academy of Marketing Science, 1674-1694. http://dx.doi.org/10.1007/BF02723327

Cabero, J., Marín, V., \& Sampedro, B. (2018). Aceptación del Modelo Tecnológico en la enseñanza superior. Revista de investigación educativa, 36(2), 435-453.

https://doi.org/10.15581/004.34.129-153

Cabero, J., \& Llorente, C. (2020). La adopción de las tecnologías por las personas mayores: aportaciones desde el modelo TAM (Technology Acceptance Model). Publicaciones, 50(1), 141-157.

https://doi.org/10.30827/publicaciones.v50i1.8521

Cabero-Almenara, J., \& Palacios-Rodríguez, A. (2020). Marco europeo de competencia digital docente «digcompedu». Traducción y adaptación del cuestionario «Digcompedu checkin». Edmetic, 9(1), 213-234.

https://doi.org/10.21071/edmetic.v9i1.124

Cabero-Almenara, J., Barroso-Osuna, J., \& LlorenteCejudo, C. (2016). Technology acceptance model, \& realidad aumentada: estudio en desarrollo. Revista Lasallista de investigación, 13(2), 18-26.

https://doi.org/10.22507/rli.v13n2a2

Cabero-Almenara, J., Guillén-Gámez, F. D., RuizPalmero, J., \& Palacios-Rodríguez, A. (2021a). Teachers' digital competence 
to assist students with functional diversity: Identification of factors through logistic regression methods. British Journal of Educational Technology.

https://doi.org/10.1111/bjet.13151

Cabero-Almenara, J., Romero-Tena, R., LlorenteCejudo, C., \& Palacios-Rodríguez, A. (2021b). Academic Performance and Technology Acceptance Model (TAM) Through a Flipped Classroom Experience: Training of Future Teachers of Primary Education. Contemporary Educational Technology, 13(3), ep305.

https://doi.org/10.30935/cedtech/10874

Çakıroğlu, Ü., Başıbüyük, B., Güler, M., Atabay, M., \& Memiş, B. Y. (2017). Gamifying an ICT course: Influences on engagement and academic performance. Computers in human behavior, 69, 98-107.

https://doi.org/10.1016/J.CHB.2016.12.018.

Chen, F., Lui, A. M., y Martinelli, S. M. (2017). A systematic review of the effectiveness of flipped classrooms in medical education. Medical Education, 51, 585-597.

https://doi.org/10.1111/medu.13272

Consejo de la Unión Europea (2018). Recomendación del Consejo, de 22 de mayo de 2018, relativa a las competencias clave para el aprendizaje permanente. Diario Oficial de la Unión Europea: Vol. C189/1. https://bit.ly/3pK1eBA

Corchuelo-Rodríguez C. A. (2018). Gamificación en la educación superior: Experiencia innovadora para motivar estudiantes y dinamizar contenidos en el aula. Revista Electrónica de Tecnología Educativa, 63, 29-41.

https://bit.ly/3dxqx3Y

Cortizo-Pérez, J. C., Carrero-García, F. M., Monsalve-Piqueras, B., Velasco-Collado, A., Díaz del Dedo, L. I., \& Pérez-Martín, J. (2011). Gamificación y Docencia: Lo que la Universidad tiene que aprender de los Videojuegos.

Davis, F. (1989). Perceived usefulness, perceived ease of use, and user acceptance of information technology. MIS Quarterly, 13(3), 319-340. https://bit.ly/3EEH0PC

De Soto-García, I. S. (2018). Herramientas de gamificación para el aprendizaje de ciencias de la tierra. Edutec. Revista Electrónica de
Tecnología Educativa, (65), 29-39. https://doi. org/10.21556/edutec.2018.65.1143

Declaración de Alejandría (2005).

http://goo.gl/UDfWX

Declaración de Estambul (2002). http://goo.gl/CjlcP

Disposición 8301, de 4 de junio de 2019, de relaciones con las cortes e igualdad (2019). Boletín Oficial del Estado, 133, sec.III, de 4 de junio de 2019, 50509. https://bit.ly/3GrEtsy

Domínguez-Díaz, A. (2018). Aportaciones sobre el uso de gamificación y redes sociales en la educación universitaria: Efectos sobre el rendimiento académico. https://bit.ly/3EEHwgw Donoso-Anés, J. A., \& López-Gavira, R. (2010). Aprendizaje basado en actividades: El uso de casos de estudio real en la asignatura de Contabilidad Financiera III. III Jornadas de Investigación e Innovación Docente, 1-18. Asociación Española de Contabilidad y Administración de Empresas, AECA.

Flores-Lueg, C., \& Roig Vila, R. (2016). Diseño y validación de una escala de autoevaluación de competencias digitales para estudiantes de pedagogía. Píxel-Bit, Revista de Medios y Educación, 12(48), 209-224.

https://doi.org/10.12795/pixelbit.2016.i48.14

Gallego-Durán, F., Villagrá-Arnedo, C. J., SatorreCuerda, R., Compañ, P., Molina-Carmona, R., \& Llorens-Largo, F. (2014). Panorámica: serious games, gamification $y$ mucho más. ReVisión, 7(2), 2. https://bit.ly/3DAyGPy

Guillén-Gámez, F. D., Cabero-Almenara, J., LlorenteCejudo, C., \& Palacios-Rodríguez, A. (2021). Differential Analysis of the Years of Experience of Higher Education Teachers, their Digital Competence and use of Digital Resources: Comparative Research Methods. Technology, Knowledge and Learning, 1-21.

https://doi.org/10.1007/s10758-021-09531-4

Gutiérrez-Priego, R., \& García-Peralta, A. (2016). ¿Cómo mejorar la calidad, la motivación y el compromiso estudiantil en la educación virtual? Campus Virtuales, 5(1), 74-82. https:// bit.ly/3dyS70B

He, T., \& Zhu, C. (2017). Digital informal learning among Chinese university students: the effects of digital competence and personal factors. International Journal of Educational 
Technology in Higher Education, 14(1), 44. https://doi.org/10.1186/s41239-017-0082-x

Herberth-Alexander, O. (2016) La gamificación como estrategia metodológica en el contexto educativo universitario. Realidad y Reflexión, 44, 29-47. https://bit.ly/3y8JyTD

Hu, L. \& Bentler, P. (1999). Cutoff criteria for fi $t$ indexes in covariance structure analysis: Conventional criteria versus new alternatives. Structural Equation Modeling: A Multidisciplinary Journal, 6(1), 1-55.

https://doi.org/10.1080/10705519909540118

Juan-Lázaro, O., \& Area-Moreira, M. (2021). Gamificación superficial en e-learning: evidencias sobre motivación y autorregulación. Pixel-Bit. Revista de Medios y Educación, 62, 146-181. https://doi. org/10.12795/pixelbit.82427

Kapp, K, M. (2012). The gamification of learning and instruction: Game-based methods and strategies for training and education. Pfeiffer.

Lai, H. M., Hsiao, Y. L., \& Hsieh, P. J. (2018). The role of motivation, ability, and opportunity in university teachers' continuance use intention for flipped teaching. Computers \& Education, 124, 37-50.

https://doi.org/10.1016/j.compedu.2018.05.013

Lévy, J. (2006). Modelización con estructuras de covarianzas en ciencias sociales: temas esenciales, avanzados y aportaciones especiales. Netbiblo.

López-Bonilla, L., \& López-Bonilla, J. (2017). Explaining the discrepancy in the mediating role of attitude in the TAM. British Journal of Educational Technology, 48(4), 940-949. https://doi.org/10.1111/bjet.12465

López-Cortés, F., Ravanal Moreno, E., Palma Rojas, C., \& Merino Rubilar, C. (2021). Niveles de representación externa de estudiantes de Educación Secundaria acerca de la división celular mitótica: una experiencia con realidad aumentada. Pixel-Bit. Revista de Medios y Educación, 62, 7-37.

https://doi.org/10.12795/pixelbit.84491

Martínez-García, E. E., Angulo-Armenta, J., \& Torres, C. A. (2020). Tendencias de la gamificación en la enseñanza: meta-análisis. Manuel Prieto, Silvia Pech y Joel Angulo, 161.

https://bit.ly/3dt3G9M
Mills, K. A. (2010). A Review of the "Digital Turn" in the New Literacy Studies. Review of Educational Research, 80(2), 246-271.

https://doi.org/10.3102/0034654310364401.

Molina-Álvarez, J. J., Ortiz-Colón. A. M., \& AgredaMontoro, M. (2017). Análisis de la integración de procesos gamificados en Educación Primaria. En Ruiz Palmero, J., SánchezRodríguez, J. y Sánchez-Rivas, E. (Eds.), Innovación docente y uso de las TIC en educación. UMA Editorial.

O’Dwyer, L. M., \& Bernauer, J. A. (2013). Quantitative research for the qualitative researcher. SAGE publications.

Ortiz-Colón, A. M., Jordán, J., \& Agredal, M. (2018). Gamificación en educación: una panorámica sobre el estado de la cuestión. Educação e Pesquisa, 44.

https://doi.org/10.1590/S1678-4634201844173773

Pelger, S., \& Nilsson, P. (2018). Observed learning outcomes of integrated communication training in science education: Skills and subject matter understanding. Studies in Educational Evaluation, 59, 195-208.

http://dx.doi.org/10.1080/21548455.2017.1417653

Pelgrum, W. J., \& Voogt, J. (2009). School and teacher factors associated with frequency of ICT use by mathematics teachers: Country comparisons. Education and Information Technologies, 14(4), 293-308.

https://doi.org/10.1007/s10639-009-9093-0.

Peñalva, S., Aguaded, I., \& de-Casas-Moreno, P. (2018). El uso de la gamificación como metodología educomunicativa en el contexto universitario. En Gamificación en Iberoamérica. Experiencias desde la comunicación y la educación (pp. 191-210). https://bit.ly/3y3PX2F

Rodríguez-García, A., \& Arias-Gago, A. R. (2020). Revisión de propuestas metodológicas: una taxonomía de agrupación categórica. Alteridad, 15(2), 146-160.

https://doi.org/10.17163/alt.v15n2.2020.01

Rodríguez-Jiménez, C., Navas-Parejo, M. R., SantosVillalba, M. J., \& Fernández-Campoy, J. M. (2019). El uso de la gamificación para el fomento de la educación inclusiva. IJNE: International Journal of New Education, 2(1), 40-59.

https://doi.org/10.24310/IJNE2.1.2019.6557 
Teo, T., Lee, C. B., Chai, C. S., \& Wang, S. L. (2009). Assessing the intention to use technology among pre-service teachers in Singapore and Malaysia: A multigroup invariance analysis of the Technology Acceptance Model (TAM). Computers y Education, 53, 10001009. https://doi.org/10.1016/j.compedu.2009.05.017

Tornero, J. P., \& Varis, T. (2010). Media literacy and new humanism. UNESCO Institute for Information Technologies in Education.

Turpo-Gebera, O., Hurtado-Mazeyra, A., DelgadoSarmiento, Y., \& Peréz-Postigo, G. (2021). Satisfacción del profesorado con la formación en servicio online: aproximaciones desde la usabilidad pedagógica. Pixel-Bit. Revista de Medios y Educación, 62, 39-70.

https://doi.org/10.12795/pixelbit.79472

Venkatesh, V., \& Bala, H. (2008). Technology acceptance model 3 and a research agenda on interventions. Decision Sciences, 39(2), 273312.

https://doi.org/10.1111/j.1540-5915.2008.00192.x

Venkatesh, V., \& Davis, A. (2000). Theoretical Extension of the Technology Acceptance Model: Four Longitudinal Field Studies. Management Science, 46(2), 186-204.

https://doi.org/10.1287/mnsc.46.2.186.11926

Villalustre-Martínez, L., \& del Moral-Pérez, M. E. (2015). Gamificación: Estrategia para optimizar el proceso de aprendizaje y la adquisición de competencias en contextos universitarios. Digital Education Review, (27), 13-31. https://bit.ly/3GhnPvQ

Williamson, B., Potter, J., \& Eynon, R. (2019). New research problems and agendas in learning, media and technology: the editors' wishlist. Learning, Media and Technology, 44(2), 87-91. https://doi.org/10.1080/17439884.2019.1614953. 


\section{EALERIDAD Percepción de las estrategias de gamificación en las escuelas secundarias italianas

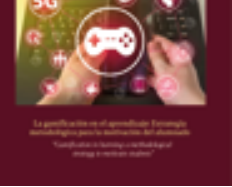 \\ Perception of gamification strategies in Italian secondary school}

(D) Viviana Malvasi es estudiante de Doctorado de la Universidad Nacional de Educación a Distancia (España) (vmalva-sil@alumno.uned.es) (https://orcid.org/0000-0001-8736-3303)

(1) David Recio-Moreno es investigador de la Universidad Nacional de Educación a Distancia (España) (davidrecio@bec.uned.es) (https://orcid.org/0000-0002-1152-6388)

Recibido: 2021-10-20 / Revisado: 2021-11-26 / Aceptado: 2021-12-13 / Publicado: 2022-01-01

\section{Resumen}

La gamificación se proyecta como un desafío para la innovación en los contextos educativos. En los últimos años se han desarrollado propuestas didácticas gamificadas para apoyar la adquisición de los contenidos curriculares de las asignaturas. Esta investigación pretende realizar un diagnóstico del nivel de aplicación de la gamificación como estrategia didáctica en el área de matemáticas, a partir de la per-cepción del profesorado y alumnado de las escuelas secundarias de Italia. Se opta por un estudio mul-ticaso, que combina técnicas cuantitativas y cualitativas, de alcance exploratorio-correlacional. Los datos se recogieron a través del cuestionario, cumplimentado por 4845 estudiantes, y de entrevistas en profundidad a 12 profesores. Se encontró que el alumnado apenas percibe el uso de juegos, analógi-cos o digitales, por parte del profesorado. El profesorado señala tener un conocimiento sobre juegos para facilitar el aprendizaje de los conceptos matemáticos. El alumnado no aprecia que se apliquen estrategias didácticas de gamificación conscientemente. El equipo docente afirma usar incentivos, pre-mios y recompensas. Al mismo tiempo, manifiestan tener un escaso conocimiento sobre la gamifica-ción y sus posibilidades. La gamificación resulta atractiva como planteamiento didáctico para la ense-ñanza de las matemáticas. En este sentido, es evidente la necesidad de ofrecer una formación sólida que siente las bases del juego, de la gamificación y las oportunidades que ofrece para el diseño de ex-periencias de aprendizaje y escenarios de juego.

Descriptores: Gamificación, estrategias didácticas, enseñanza de las matemáticas, educación secundaria, proceso de aprendizaje, método de enseñanza.

\section{Abstract}

Gamification is projected as a challenge for innovation in educational contexts. In recent years, ga-mified didactic proposals have been developed to support the acquisition of the curricular contents of the subjects. This research aims to make a diagnosis of the level of application of gamification as a didactic strategy in the area of mathematics, based on the perception of teachers and students of se-condary schools in Italy. A multi-case study is chosen, which combines quantitative and qualitative techniques, with an exploratory-correlational scope. The data was collected through the questionnai-re, completed by 4,845 students, and in-depth interviews with 12 teachers. It was found that the stu-dents hardly perceived the use of games, analogue or digital, by the teaching staff. The teachers indi-cate that they have knowledge of games to facilitate the learning of mathematical concepts. Students do not perceive that gamification didactic strategies are applied consciously. The teaching team claims to use incentives, prizes, and rewards. At the same time, they claim to have little knowledge about gamification and its possibilities. Gamification is attractive as a didactic approach to teaching mathematics. In this sense, the need to offer solid training that establishes the foundations of the ga-me, gamification and the opportunities it offers for the design of learning experiences and game sce-narios is highlighted.

Keywords: Gamification, didactic strategies, teaching math, secondary education, learning processes, teaching methods.

Forma sugerida de citar: Malvasi, V., \& Recio-Moreno, D. (2022). Percepción de las estrategias de gamificación en las escuelas secundarias italianas. Alteridad, (17)1, 50-63. https://doi.org/10.17163/alt.v17n1.2022.04 


\section{Introducción y estado de la cuestión}

A lo largo de los últimos años, el concepto de juego se ha proyectado en distintos contextos como la publicidad y el marketing (Huotari \& Hamari, 2012; Landers et al., 2017; Zichermann \& Linder, 2013), la salud (González et al., 2016; Hamari \& Koivisto, 2015) o la educación (Domínguez et al., 2013; Qahri-Saremi \& Turel, 2016; Villagra-Arnedo et al., 2016). Este panorama, generado por la creciente producción de la literatura científica en estos contextos (Contreras \& Eguia, 2016; Chou, 2021; Johnson et al., 2016; Kocakoyun \& Ozdamli, 2018; Koivisto \& Hamari, 2019; Sardi et al., 2017), ha provocado una confusión general sobre la definición de la gamificación (Torres-Toukoumidis et al., 2018).

Desde que el término gamificación empezó a utilizarse hasta la actualidad, no hay un acuerdo entre la comunidad científica a la hora de establecer una definición común, ya que cada autor presenta un enfoque particular y hace hincapié en unos aspectos determinados. Entre las definiciones más populares, se entiende la gamificación como el uso de mecánicas de juego en un contexto no lúdico (Deterding et al., 2011), como el uso de elementos de juego y técnicas propias del diseño de juegos en entornos no lúdicos (Werbach \& Hunter, 2012), como un conjunto de herramientas empleadas en iniciativas de marca mediante elementos y mecánicas de juego (Zichermann \& Linder, 2013) o, por último, la gamificación considerada como el uso de elementos de diseño de juego en contextos de no lúdicos (Deterding et al., 2011).

Otras definiciones están enfocadas en la experiencia de los usuarios. En ellas, la gamificación se ve como un proceso para mejorar un servicio a través de experiencias de juego con la finalidad de asistir a los usuarios para generar un valor general (Huotari \& Hamari, 2012) y como un uso deliberado de elementos de juego para conseguir una experiencia de juego a partir de actividades en contextos no lúdicos (Seaborn
\& Fels, 2015). Estos enfoques se centran en las actividades que deben realizar las personas para adquirir un aspecto similar al de un juego, es decir, que las identifiquen como un juego en un ambiente lúdico.

Por último, en relación con las diferentes definiciones que se pueden encontrar en torno a la gamificación, se abordan planteamientos centrados en la conducta y el comportamiento de las personas. En estos casos, la gamificación se comprende como la aplicación de estrategias, pensamientos y mecánicas de juegos en contextos no lúdicos, apartados de juegos, con el fin de que las personas adopten o mantengan determinados comportamientos (Ramírez, 2014). En esta línea, Kapp (2012) define la gamificación como el uso de mecánicas de juego, percepciones y pensamientos de juego para involucrar a las personas, motivarlas a la acción, apoyar su aprendizaje y resolver problemas. Zichermann y Cunningham (2011) apuestan por entenderla como un proceso de pensamiento y mecánicas de juego para conectar con las personas y resolver problemas. Dados los innegables beneficios de esta estrategia didáctica, actualmente se están desarrollando métodos para su aplicación. Por ejemplo, Gasca-Hurtado et al. (2017) presentan una propuesta para la evaluación de entornos gamificados con el fin de asegurar que las experiencias gamificadas cumplan con propósitos, objetivos, principios y elementos que se definen como componentes fundamentales de la gamificación. Es decir, su propuesta está orientada a la definición de un método de diseño para actividades gamificadas.

La revisión bibliográfica llevada a cabo muestra un escaso abordaje de la gamificación como estrategia didáctica en la enseñanza de las matemáticas. Si bien es cierto, existen diferentes investigaciones o experiencias educativas que presentan los resultados obtenidos de la implementación de la gamificación en la etapa de educación primaria y secundaria (FernándezGavira et al., 2018; Pisabarro \& Vivaracho, 2018; Quintero et al., 2018), y en educación superior 
(Corchuelo, 2018; Fernández-Antolín et al., 2021; Santos-Ferreira \& Lacerda-Santos, 2018; PérezLópez et al., 2017). Sin embargo, se encuentran distintos estudios que relacionan la gamificación con la enseñanza de las matemáticas. Tomislav et al. (2018) señalan que las actividades ludificadas contribuyen a incrementar el rendimiento del alumnado en el aprendizaje de los conceptos matemáticos. Otro estudio presenta un proyecto educativo dirigido al alumnado para ayudar a mejorar su percepción ante el fracaso en el área de matemáticas mediante la incorporación de un juego interactivo de misterio, como estrategia didáctica, en el que se trabajan distintos contenidos matemáticos (Carson, 2021). Por su parte, Widodo y Rahayu (2019) muestran los resultados de un estudio donde los y las estudiantes, tras utilizar juegos para trabajar conceptos aritméticos, donde muestran un mayor compromiso y una mayor conexión emocional. En esta línea, López et al. (2021) analizan las opiniones del profesorado del área de matemáticas, sobre todo en relación con las competencias STEM, en centros educativos brasileños y españoles. En este estudio, un alto porcentaje de los docentes piensan que las actividades gamificadas tienen una implicación positiva en el aprendizaje del alumnado, favoreciendo un mayor acercamiento hacia las matemáticas y hacia las habilidades vinculadas con esta competencia matemática. Por último, Zaharin et al. (2021) exploran las percepciones de estudiantes sobre la aceptación, el interés y las habilidades a la hora de implementar la gamificación en los procesos de aprendizaje de las matemáticas. Los resultados muestran una aceptación elevada al considerar la gamificación como una estrategia didáctica que beneficia el aprendizaje de los conceptos matemáticos específicos. Esta conexión entre el juego y el aprendizaje se plantea como una línea de actuación para trabajar los contenidos curriculares del área de matemáticas.

Si se extrapolan todos estos planteamientos al ámbito educativo, la gamificación se puede entender como una estrategia didáctica que puede enriquecer los planteamientos metodológicos del profesorado. An et al. (2021) analizan las percepciones del profesorado en relación con el interés, eficacia, barreras percibidas y necesidades respecto a los enfoques gamificados dentro de la metodología docente, en particular en los modelos MOOC (Massive Online Open Courses). En este caso, el profesorado muestra un interés elevado hacia la gamificación y los elementos del juego, motivado por conseguir una mayor interacción y conexión con el alumnado. Entre las principales barreras se encuentra la falta de tiempo, el conocimiento de la temática, la falta de financiación y la escasa relación entre los contenidos específicos y el enfoque gamificado en el planteamiento didáctico, precisando una capacitación por parte de expertos.

Lejos de planteamientos simplistas de la gamificación, se hace necesario un enfoque global que tenga muy en cuenta la esencia del juego, así como la cohesión entre las dinámicas, los elementos y las mecánicas que lo integran. Para ello, se precisa de una formación consistente para evitar el sentimiento de inseguridad y la necesidad de una formación específica para afrontar con garantía planteamientos metodológicos basados en la gamificación (López et al., 2021). En esta línea, a la hora de afrontar el diseño de una experiencia lúdica de aprendizaje, existen diferentes elementos que hay que tener en cuenta. Siguiendo a Werbach y Hunter (2012), cabría encontrar un sentido al momento de incorporar un conjunto de dinámicas (narrativa, aspectos emocionales, reglas, límites, progresión o interacciones), mecánicas (obtención de recompensas y premios, retroalimentación, desafíos o retos, competición, cooperación o colaboración) y componentes (personajes o avatares, insignias, puntuaciones, clasificaciones, niveles, equipos, combates o competiciones). Todo ello unido a una serie de objetivos que el profesorado precisa definir en el momento inicial, que guiará la fase de diseño y la evaluación final de la experiencia gamificada que decida desarrollar en un área y una etapa específica. Para concluir, 
en un nivel más general, Hossein-Mohand et al. (2021) sostienen que modelos pedagógicos como el flipped learning y las metodologías activas como el aprendizaje basado en proyectos y la gamificación, facilitan los procesos de enseñanza y aprendizaje de las matemáticas, mediante el apoyo también de tecnológicos que favorece el intercambio de información y contenidos, la participación y la colaboración entre el alumnado.

Este es el contexto de este estudio, que trata de descubrir la percepción, tanto del alumnado como del profesorado, sobre el conocimiento de la gamificación como estrategia didáctica en la enseñanza de los contenidos curriculares del área de matemáticas en las escuelas secundarias de Italia.

\section{Método}

La propuesta metodológica que sigue este estudio multicaso (Stake, 2005) pretende explorar más de una unidad de análisis para proporcionar las bases para su generalización (Rule \& Mitchell, 2015). Esta opción aporta criterios de validez interna, externa y confiabilidad de los datos recogidos. En este estudio multicaso, la combinación de técnicas cuantitativas y cualitativas se presenta mediante un diseño mixto de alcance exploratorio-correlacional. En relación con el diseño cuantitativo, el instrumento que se utiliza para la recopilación de los datos es el cuestionario, cumplimentado por un total de 4845 estudiantes adolescentes que residen en 75 de las 80 provincias que conforman la organización territorial de Italia. El cuestionario es elaborado con preguntas y respuestas concretas, a las que se añaden preguntas abiertas y de elección múltiple. La metodología de carácter cualitativo (Stake, 2007; Barton et al., 2009; Igartua \& Humanes, 2009; Gibbs, 2012) se centra en el análisis del discurso (Valles, 2000; Iñiguez, 2006; Van Dijk, 2005) de 12 entrevistas en profundidad al profesorado del área de matemáticas que desarrolla su docencia en las escuelas secundarias.
Con el fin de definir el propósito del estudio, se plantean los siguientes interrogantes dirigidos al alumnado: ¿el profesorado propone juegos digitales en las clases de matemáticas?, ¿el profesorado plantea juegos serios (analógicos)?, ¿qué juegos empleados por el profesorado han ayudado a una mejor comprensión de los contenidos del área de matemáticas?, ¿conoce juegos (analógicos o digitales) que se podrían utilizar en las clases de matemáticas?, ¿el profesorado utiliza incentivos, premios o recompensas en la asignatura de matemáticas por realizar correctamente las tareas escolares fuera del aula?, ¿el profesorado de matemáticas usa incentivos, premios o recompensas por hacer bien las actividades propuestas dentro del aula?

En relación con las acciones que realiza el profesorado, se formulan las siguientes cuestiones: ¿qué entiende por gamificación?, ¿cómo valora la gamificación en cuanto a su incorporación en su metodología docente?, ¿ha recibido formación relacionada con esta estrategia didáctica?, ¿conoce o propone algún juego (analógico o digital) a su alumnado para ayudar o facilitar el aprendizaje de los contenidos del área de matemáticas?, ¿utiliza incentivos, premios o recompensas por el trabajo realizado por su alumnado?, ¿usa alguna aplicación digital para otorgar este tipo de premios, incentivos o recompensas? Utilizar el juego en la práctica educativa es valorado como una forma de acostumbrar al alumnado a recibir en todo momento recompensas por lo que realiza y no esforzarse, ¿qué opina sobre esta afirmación?

\subsection{Objetivos e hipótesis}

Las preguntas anteriores convierten una reflexión inicial en el objetivo de este estudio. Formalmente, la investigación pretende diagnosticar el nivel de aplicación de la gamificación como estrategia didáctica en el área de matemáticas, a partir de la percepción tanto del profesorado como del alumnado en las escuelas secundarias de Italia. La formulación de este 
objetivo responde a un estudio que se proyecta para aproximarse a los fenómenos observables. A continuación se presentan varias hipótesis que han sido contrastadas y formuladas mediante un método hipotético-deductivo, relacionadas con el objetivo:

- Hipótesis 1. En la etapa de educación secundaria de los centros escolares de Italia, la gamificación como estrategia didáctica en el área de matemáticas es percibida por parte del alumnado.

- Hipótesis 2. En la etapa de educación secundaria de los centros escolares de Italia, la gamificación como estrategia didáctica en el área de matemáticas es conocida por parte del profesorado.

- Hipótesis 3. En la etapa de educación secundaria de los centros escolares de Italia, la gamificación como estrategia didáctica en el área de matemáticas es aplicada por parte del profesorado.

\subsection{Población y muestra}

Las personas participantes en la muestra son 4845 estudiantes en escuelas secundarias, residentes en Italia, con edades comprendidas entre los 13 y 22 años, y con una media de 16,43 años. El $52 \%$ de las personas encuestadas se identifican con el género femenino y el $48 \%$ con el masculino. Las provincias de residencia alcanzadas son 75 de un total de 80, distribuidas en distintas zonas geográficas de Italia: norte $(60$ $\%)$, centro $(7 \%)$, sur e islas (33\%). Todas estas personas residen en más de 800 localidades diferentes, el $66,6 \%$ en una zona rural y el 33,4\% en una zona urbana. La muestra reducida de la zona centro no fue intencional, sino que responde a una solicitud de participación de dirigentes de las tres áreas del país, aunque, en su mayoría, no suministran el cuestionario por la situación del alumnado durante la crisis provocada por la COVID-19.
El equipo docente de disciplinas científicas que participa en las entrevistas está formado por 12 personas. Un $17 \%$ de las personas entrevistadas se identifica con el género masculino, mientras que un $83 \%$ lo hace con el femenino. Las edades están comprendidas entre los 32 y 59 años. Cuentan con una experiencia docente entre los 2 y 33 años. A nivel formativo, la muestra posee un enorme potencial para el estudio, y es de vital importancia que el $42 \%$ de las personas entrevistadas estén graduadas en Matemáticas, el $25 \%$ en Ciencias Estadísticas, el $17 \%$ en Física y el $8 \%$ respectivamente en Economía e Ingeniería Informática, respondiendo así a todos los ámbitos de las competencias STEM. Geográficamente, el $58 \%$ reside y desarrolla su actividad profesional en la zona norte de Italia, el $8 \%$ vive en el centro y, por último, el $34 \%$ en la zona sur e islas.

Cabe destacar que, el itinerario escolar está dividido en tres etapas: la escuela primaria (que está compuesta por un total de cinco cursos dirigidos al alumnado con una edad comprendida entre los 6 y los 11 años), la escuela secundaria de primer grado (que está formada por tres cursos y alumnado con edades situadas entre los 11 y los 14 años) y la escuela secundaria de segundo grado (que se encuentra organizada en cinco cursos y el alumnado tiene una edad comprendida entre los 14 y 19 años). Este camino escolar está estructurado por un total de 13 cursos académicos, de los cuáles los diez primeros son obligatorios. Este es el contexto de este estudio, enfocado en la etapa de la escuela secundaria de segundo grado. La motivación principal para dirigir esta investigación a dicha etapa se fundamenta en los bajos resultados que obtiene el alumnado en el área de matemáticas, según las pruebas nacionales establecidas (Invalsi, 2019).

\subsection{Instrumentos}

En la parte cuantitativa del estudio se opta por el diseño de un cuestionario estructurado en preguntas cerradas, abiertas y de múltiple respuesta. Las variables independientes son: género, edad, 
macroárea, tipología de centro escolar, curso y rendimiento académico. Como variables dependientes se consideran las distintas perspectivas que permiten responder a los interrogantes, objetivos e hipótesis de la investigación.

Para afrontar el planteamiento cualitativo se apuesta por el diseño de una entrevista semiestructurada dirigida a los observadores privilegiados, recogiendo información de carácter personal como el género, la edad, la ciudad en la que imparte su enseñanza, la tipología de centro educativo, los años de experiencia y, en último lugar, el grado de formación. Se analizan también los métodos didácticos más utilizados por el profesorado y las principales dificultades encontradas en su implementación. Mediante la herramienta Google Forms se elabora el consentimiento informado para que las personas entrevistadas dieran su aprobación para su grabación y posterior análisis. Durante la entrevista, se favorece que las personas aporten sus respuestas de manera libre, evitando interrumpir sus intervenciones, aunque en ciertos momentos se opta por incidir sobre algunos aspectos para conseguir que el equipo docente ofrezca una respuesta más clara. Para realizar referencias ágiles y simples a la entrevista, y diferenciar las aportaciones de cada una de las personas entrevistadas, se opta por la codificación: "E-Tno: pág". En este sistema de codificación, se hace referencia a la entrevista (E), al profesor (T), al número de entrevista específica $\left(\mathrm{n}^{\circ}\right)$ y a la página en la que se localiza el hecho referido (pág). Por lo tanto, si durante el análisis se requiere reportar una información que aparece en la primera página de la entrevista al participante 1 , quedaría codificado de la siguiente manera: E-T1:1.

\subsection{Procedimiento}

El diseño metodológico cuantitativo, correspondiente a los cuestionarios aplicados a alumnado italiano de segundo nivel de la escuela secundaria, y el diseño metodológico cualitativo, centrado en las entrevistas semiestructuradas a docentes de secundaria, facilitan el análisis desde ambas perspectivas que enriquecen el proceso; ambos se desarrollaron entre marzo 2020 y mayo 2020. Se aplican, por tanto, metodologías mixtas propias de las ciencias sociales al combinar en un solo estudio técnicas de investigación, métodos, enfoques, conceptos o lenguaje cuantitativo o cualitativo (Johnson \& Onwuegbuzie, 2004). Esto posibilita adquirir una mayor comprensión de lo que estamos estudiando, abarcando las fortalezas de ambas metodologías y analizando diferentes enfoques, combinando los datos para obtener resultados convergentes (Callejo \& Viedma, 2006).

En cuanto a la elaboración de los cuestionarios, se genera un formulario enviado a los centros educativos italianos, pidiendo su colaboración para esta investigación. Una vez obtenidos los datos necesarios, son analizados siguiendo el correspondiente procedimiento. En primer lugar, para el análisis cuantitativo se exportaron los datos .csv a un archivo Excel para su posterior interpretación con el programa estadístico $I B M$ SPSS $v$. 25. Se introducen los datos en el programa, asignando valores numéricos a las respuestas obtenidas en los cuestionarios, procediendo al filtrado y depuración, seleccionando el procedimiento adecuado para calcular los estadísticos y ejecutando el procedimiento con el fin de obtener los informes de investigación. Las entrevistas se desarrollan mediante la herramienta Skype y, posteriormente, se realiza la transcripción, recopilación y análisis de datos relacionados con los objetivos e hipótesis. Finalmente, se establece una triangulación metodológica y comparación de los datos de la encuesta con los resultados del análisis del discurso que se presentan en este estudio, respetando a todas las personas participantes.

\subsection{Fiabilidad y validez}

Este criterio determina si las escalas de medidas de nuestra herramienta son fiables, entendiendo la fiabilidad como la precisión del instrumento, atendiendo a los posibles errores encontrados 
en el análisis factorial. Si los errores que se presentan son menores, mayor es la precisión de la medida y, por tanto, del estudio. Para estudiar la fiabilidad del instrumento hemos seguido el procedimiento del $\alpha$ de Cronbach. Tras analizar las estadísticas de fiabilidad, el $\alpha$ de Cronbach ofrece un valor de 0,65 referida a los ítems sobre gamificación, lo que confirma una suficiente fiabilidad.

\section{Resultados}

A continuación, se presentan los resultados obtenidos en el estudio organizados en tres categorías:
Categoría 1. Clases de matemáticas: juegos propuestos versus juegos conocidos

A la cuestión relacionada con la propuesta de juegos analógicos o digitales en las sesiones de matemáticas, el alumnado contesta negativamente, con un $94 \%$ y $95,5 \%$ respectivamente. En cuanto a las preguntas sobre el conocimiento de juegos que favorezcan la comprensión de los contenidos de la asignatura de matemáticas según el alumnado, las respuestas denotan un posicionamiento negativo en un $83 \%$, mientras que el $6 \%$ indica que son juegos analógicos (juegos de cartas, juegos de mesa, sudokus y ajedrez). Un $5 \%$ señala que son juegos digitales, pero no aportan ejemplos, el $4 \%$ apunta a Kahoot y el 2 $\%$ las competiciones (Figura 1).

\section{Figura 1. Juegos que favorecen la comprensión de los conocimientos matemáticos según el alumnado}

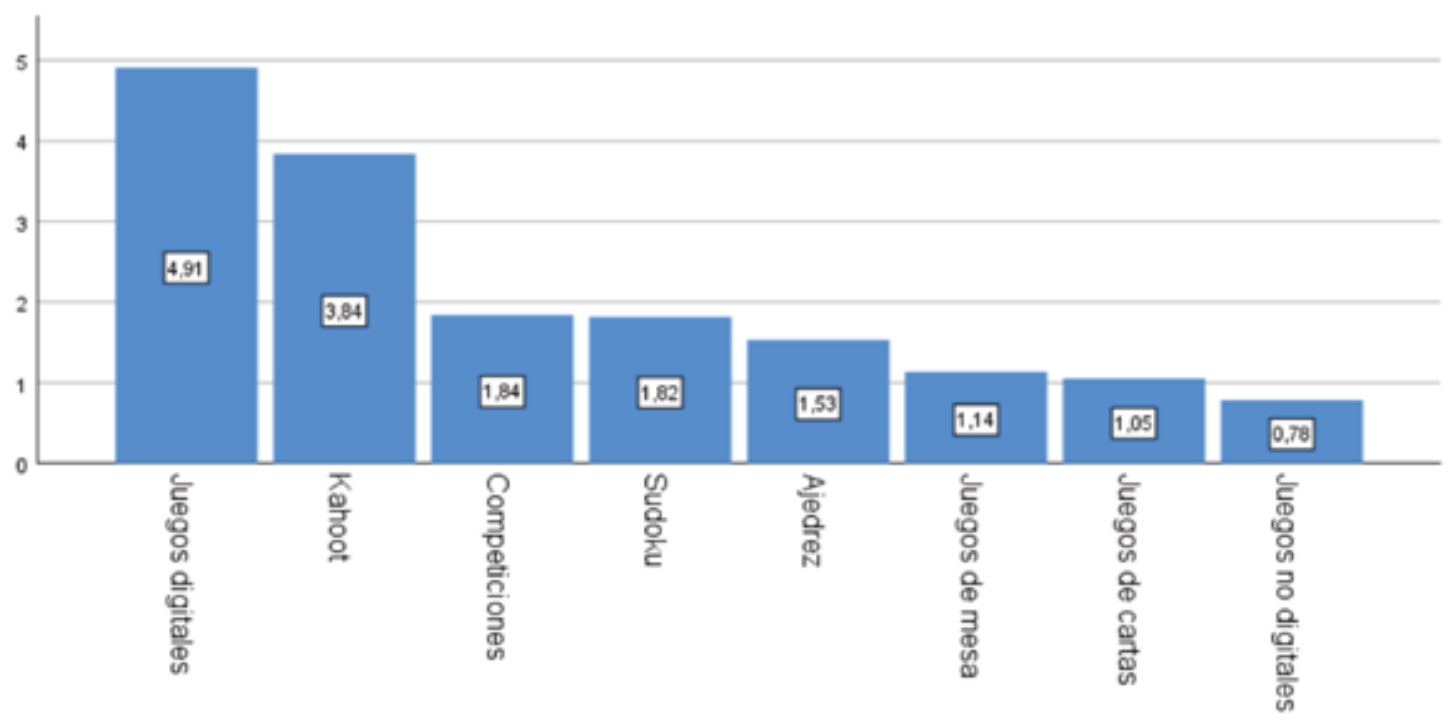

A pesar de la respuesta negativa del alumnado ante la cuestión sobre si el profesorado propone juegos de tipo analógico o digital durante las sesiones de matemáticas, al plantear la pregunta al profesorado sobre si conoce o propone algún juego analógico o digital a su alumnado que facilite el aprendizaje de las matemáticas, el $75 \%$ contesta positivamente haciendo referencia a juegos de adivinanzas, competiciones entre equipos, concursos o premios.

Tomamos juegos como acertijos o sudokus. (E-T1: 6)

Organizo concursos de premios o competiciones de velocidad en el cálculo. (E-T2: 10)

Usamos Scratch. Y luego propongo sudokus cuando hay pocos en la clase. (E-T3: 15) 
Digital no, no digital el Sudoku porque carecemos de herramientas. (E-T4: 20)

Juegos de lógica. (E-T5: 24)

Juegos de las olimpiadas de matemáticas. (E-T7: 32)

Durante los períodos de suspensión de las actividades didácticas y en esta fase de DAD. (E-T8: 35)

Divertidos problemas y acertijos. (E-T9: 39)

Digital no, suelo hacer pequeños concursos tratando de estimularlos, competiciones, sin premios. En cuarto grado quería hacer una competición con las derivadas similar a una competición que hice que se llamaba 'Don't drink and derive’ y parecían felices. (E-T10: 44)

La diferencia en las respuestas entre el alumnado y el profesorado puede deberse en el significado del juego por el alumnado.

Categoría 2. Percepción de la aplicación de las estrategias didácticas de gamificación según el alumnado

Para descubrir el nivel de aplicación de las estrategias didácticas de gamificación en el área de matemáticas, se combinan las respuestas del alumnado con las respuestas del profesorado. Avanzando en la incorporación de la gamificación en las aulas, entendiéndose no solo como la integración de juegos sino como un cambio metodológico hacia un escenario y experiencia de juego, se pone de manifiesto que el alumnado contesta de forma negativa en un $81 \%$ y $77 \%$ a que el profesorado utilice incentivos, premios y recompensas por realizar correctamente las tareas fuera del ámbito escolar o por hacer bien los ejercicios y problemas en el aula. Los datos recogidos revelan que el nivel de percepción de la aplicación de estrategias didácticas de gamificación en los centros educativos de segundo grado de la etapa secundaria es bajo por parte del alumnado.

\section{Categoría 3. Aplicación de la gamificación como estrategia didáctica por parte del profesorado}

A la pregunta relacionada sobre el concepto de gamificación, el $67 \%$ contesta que no sabe en qué consiste y por eso no puede expresar una opinión, o bien porque con lo extensos que son los programas ministeriales y la reducción de las horas de dedicación, apenas hay tiempo para abordar planteamientos innovadores.

Hay poco tiempo para probar nuevas soluciones educativas, considerando también las numerosas salidas educativas en la zona. (E-T3: 15)

No lo sé, pero pretendo estudiarlo. (E-T9: 39)

No lo sé, no la conozco. (E-T2: 10; E-T6: 28; E-T7: 31; E-T10: 44; E-T11: 47; E-T12: 50)

La totalidad de las personas entrevistadas contestan negativamente en cuanto a haber recibido formación específica acerca de la gamificación y su uso como estrategia didáctica para la enseñanza de las matemáticas, aunque el equipo investigador considera que se realiza como una gamificación inconsciente o invisible, es decir, que el profesorado la aplica sin ser consciente de ello. De hecho, a la cuestión dirigida al profesorado sobre si emplea incentivos, recompensas o premios por el trabajo realizado por sus estudiantes, el grupo contesta afirmativamente en un $75 \%$.

Sensación de gratificación de un comentario positivo, una palmada en la espalda para felicitarlos, a este nivel sí pero de recompensas más materiales no. (E-T1: 6)

De forma lúdica, sí. A veces utilizo premios. Por ejemplo, dejo decidir a quién interrogar (broma) u ofrezco la inmunidad por aquel día a preguntas como premio. Esto aligera el clima en el aula, fortalece al grupo dentro del aula. (E-T2: 10) 
Dividí la clase en tres grupos y jugué el clásico juego de preguntas. Quien respondió primero fue recompensado ( $i y$ yo traje dulces como recompensa!). Luego, cuando explico y asigno los ejercicios, a veces propongo un ejercicio muy difícil y el primero que lo hace bien toma 8 como nota oral (claro que les advierto antes). (E-T3: 15)

Utilizo recompensas en términos de votos o menos ejercicios. Tanto positivo como negativo: si terminas último eres penalizado. (E-T4: 20)

Sí, generalmente uso incentivos. También este año participé en un proyecto interdisciplinario con una segunda clase. Los chicos, divididos en las casas de la película de Harry Potter, tenían que presentar cada semana los trabajos asignados, recibiendo puntos por sus casas. Se planearon premios para finales de año. No utilizo aplicaciones digitales para obtener recompensas o premios, pero todavía me parece estimulante recompensar a los chicos con incentivos que luego se convierten en un voto oral o un aumento en la calificación de la prueba escrita. (E-T5: 24)

No utilizo recompensas materiales, sin embargo, en ocasiones, animo el logro de objetivos con promesas de calificaciones (a lo que me doy cuenta de que los chicos están mucho más apegados que yo como docente). Es frecuente, sin embargo, que organizo desafíos en grupos pequeños o individuales en clase con premios "culinarios" ofrecidos al ganador por los otros compañeros. (E-T8: 36)

Sí, lo tengo en cuenta en la evaluación de forma sistemática. Creo que esta metodología es útil especialmente cuando tienes una audiencia de chicos pequeños, estoy pensando en el bienio, los primeros dos años. Con chicos mayores y más maduros ya no es necesario. (E-T9: 39)

Todavía no lo he puesto en práctica, pero me gusta este método. Incluso a nivel escolar, competiciones entre clases. Aprecio el hecho de que da más participación. Francamente, no veo cosas negativas si se toma en el justo modo la cosa. (E-T10: 44)

No, pero si hacen algo extremadamente mal pongo dos, ¿es válido? (E-T11: 48)

Los datos recopilados en la fase cualitativa del estudio, dirigida al profesorado, señalan que cada uno utiliza recompensas o premios diferentes, no materiales, que quizá el alumnado no percibe como tal. Además, no se comparte ninguna aplicación digital para facilitar el uso de estos elementos durante el proceso.

Para profundizar en la concepción del equipo docente sobre la gamificación, se propone una reflexión sobre la valoración de la utilización del juego como un medio para dirigir e instruir al alumnado en la recepción continua por aquellas acciones que realizan y disminuir con ello su esfuerzo. El $67 \%$ de las personas entrevistadas declara no estar de acuerdo con estos postulados, considerando que, con la introducción del juego en los procesos de enseñanza, esforzarse es más divertido.

El juego estimula la competitividad, la formación del carácter, el respeto a las reglas y roles, el conocimiento de uno mismo y de los demás. El método Scout, un método pedagógico muy conocido, se basa en el juego. Los que dicen que el juego acostumbra a no esforzarse, quizás, nunca ha jugado en su vida. (E-T2: 10)

No veo que los estudiantes aprendan por aprender, ya están estudiando para la votación. Ya es así 'do ut des'. (E-T3: 15)

No siempre obtienes una recompensa, o la recompensa también puede ser solo una gratificación verbal. (E-T4: 20)

En la escuela secundaria de segundo grado, sin embargo, creo que el chico o la chica deben haber adquirido ya una madurez para comprender que el esfuerzo realizado es en beneficio de su futuro y no para recibir una 
recompensa. El uso de juegos en las prácticas educativas debe ser un plus, pero no la base. (E-T5: 25)

Podría ser útil si se combina con el método tradicional; en el día a día no funciona así, hay que acostumbrarse a esforzarse para conseguir algo. (E-T6: 28)

El uso del juego en la enseñanza podría ser beneficioso, pero hay que tener cuidado. Creo que lo ideal no es convertirlo en el único enfoque, sino alternar el juego con momentos de trabajo sin él. (E-T7: 32)

No estoy de acuerdo con esta afirmación, ya que supongo que la recompensa debe otorgarse solo cuando la meta se alcance por completo. (E-T8: 36)

No estoy de acuerdo con estos estudios porque para participar en la competición y jugar hay que saber, hay que esforzarse. (E-T10: 44).

Como se puede observar, la mitad del equipo docente evalúa positivamente la introducción del juego (o bien de sus mecánicas) en el acto didáctico, mientras no sea el único enfoque o modelo educativo.

\section{Discusión y conclusiones}

Los resultados encontrados en esta investigación permiten una aproximación al nivel de aplicación de la gamificación como estrategia didáctica en el área de matemáticas en las escuelas secundarias de Italia. Al mismo tiempo, permiten destacar algunas conclusiones y establecer unas líneas futuras de investigación.

En relación con el uso de juegos en las sesiones de matemáticas, el alumnado no aprecia que el profesorado utilice juegos, tanto analógicos como digitales, en el aula. Tampoco poseen un conocimiento sobre juegos que favorezcan la adquisición de los contenidos de la asignatura de matemáticas. Hacen mención a aplicaciones como Kahoot, que son utilizadas de forma esporádica en algún momento del proceso de formación. Por otro lado, el profesorado afirma conocer diferentes tipos de juegos como medio para facilitar el aprendizaje de los conceptos del área. Señalan juegos como las adivinanzas, competición entre equipos, concursos, entre otros. Este tipo de actividades gamificadas, como indican Tomislav et al. (2018), incrementan el rendimiento en relación con el aprendizaje de los conceptos matemáticos concretos.

Abordando la percepción de la aplicación de la gamificación como estrategia didáctica, el alumnado señala que no aprecia que se utilicen estrategias gamificadas en las sesiones de matemáticas. Directamente, no perciben el uso de incentivos, premios y recompensas a cambio de realizar adecuadamente las tareas escolares, tanto dentro como fuera de las aulas, así como los ejercicios y problemas planteados en el aula. Por el contrario, Zaharin et al. (2021), en su exploración realizada sobre la percepción del alumnado, mostraban una elevada aceptación e interés ante las habilidades desarrolladas mediante la incorporación de la gamificación como estrategia para el aprendizaje de los conceptos matemáticos.

En cuanto a la aplicación de estrategias didácticas de gamificación por parte del profesorado, el equipo docente manifiesta un escaso conocimiento sobre la gamificación y las oportunidades que ofrece a su ejercicio profesional. Sostienen no abordar la gamificación en sus planteamientos por la falta de tiempo, las horas de dedicación que supone y por la situación excepcional provocada por la COVID-19, entre otros motivos. Este motivo se encuentra alineado con las conclusiones de López et al. (2021), cuando se hace referencia a una mayor capacitación en los planteamientos metodológicos basados en la gamificación para aumentar la seguridad del profesorado y conectar los contenidos curriculares del área a través de las estrategias de gamificación. También mencionan no haber recibido formación específica sobre la gamificación y su implementación en la enseñanza de las matemáticas. Aunque muchos profesores no son 
conscientes de ello, comentan utilizar premios, incentivos o recompensas en su acción docente. Es decir, no son conscientes de emplear estos elementos en sus planteamientos. El alumnado tampoco es consciente del uso de estos elementos. En este sentido, como mantienen An et al. (2021), se hace necesaria una capacitación por parte de expertos en gamificación que permita apoyar con rigor los planteamientos didácticos en el área de las matemáticas, así como proporcionar mayor tiempo y flexibilidad a sus propuestas, financiación, ejemplificación y recursos que apoyen la incorporación de elementos propios del juego en las prácticas docentes.

Se percibe un uso limitado de algunos elementos de la gamificación, que se encuentran estrechamente relacionados con mantener o adquirir ciertos comportamientos o conductas en su alumnado. Esta idea se encuentra alineada con las aportaciones de Ramírez (2014), sobre todo cuando el uso de estas estrategias se dirige a que las personas adopten o mantengan determinados comportamientos. La mayoría del profesorado no está de acuerdo en dar valor al juego como un medio para instruir al alumnado en la recepción de recompensas únicamente y no favorecer así su esfuerzo. En este sentido, la aplicación de las estrategias didácticas de gamificación, implica una integración del juego y de un cambio metodológico hacia el diseño de experiencias y escenarios de juego. Sostienen que es un recurso, pero no es un modelo educativo.

En el momento actual, en el que se observan multitud de experiencias didácticas gamificadas desarrolladas por profesionales del campo educativo, surge la necesidad de evitar la confusión que se puede generar en torno al concepto de gamificación y conseguir así un consenso claro sobre su definición, aspecto que señalan Torres-Toukoumidis et al. (2018). Esta cuestión se relaciona con el reto de ofrecer una formación sólida al profesorado que facilite un diseño consistente de experiencias lúdicas de aprendizaje para ser llevadas a cabo en distintas áreas y etapas educativas, todo ello en la línea que mantienen Werbach y Hunter (2012), cuando hacen referencia a la incorporación coherente de un conjunto de dinámicas, mecánicas y componentes del juego en el diseño de las diferentes propuestas educativas en el área de matemáticas.

A modo de conclusión, y con el objetivo de establecer unas líneas de actuación e investigación, la gamificación se define como el uso de elementos, mecánicas y dinámicas propias de los juegos en un entorno no lúdico, tal y como definen diferentes autores en sus trabajos como (Deterding et al., 2011; Zichermann \& Linder, 2013). Existen experiencias educativas basadas en un sistema gamificado que únicamente utiliza puntos, medallas y clasificaciones. Estos planteamientos sitúan a la gamificación en un planteamiento reduccionista y limitado, al contrario del postulado de Kapp (2012), cuando se trata de involucrar, motivar al alumnado a la acción, apoyar su aprendizaje y resolver distintos problemas. A la hora de diseñar experiencias didácticas lúdicas en los contextos escolares, se debe considerar que este planteamiento no debe ser único sino que se puede enriquecer la propuesta mediante la incorporación de otros elementos como una situación inicial o problema que contextualicen la experiencia, una narrativa que sirva como hilo conductor y ambientación (personajes, escenarios, hechos, acontecimientos, etc.), un planteamiento de retos diversos que favorezcan la acción, colaboración y participación del alumnado para resolver problemas (Zichermann \& Cunningham, 2011). En esta línea se encuentra el trabajo realizado por Hossein-Mohand et al. (2021) en el que mantienen que modelos pedagógicos como el flipped classroom, las metodologías activas y la gamificación favorecen los procesos de enseñanza y aprendizaje de las matemáticas, unido al apoyo que los recursos tecnológicos pueden ofrecen en las prácticas educativas.

Este enfoque innovador se convierte en un verdadero desafío para el profesorado que trata de proyectar el juego en los contextos educativos y lo vincula con el aprendizaje de los contenidos curriculares de una materia (Domínguez et al., 
2013; Qahri-Saremi \& Turel, 2016; VillagraArnedo et al., 2016), y también para las instituciones de formación universitaria, para tratar de alcanzar una formación sólida que ayude al diseño y desarrollo de propuestas lúdicas que traten de unir el aprendizaje de los contenidos curriculares con la diversión.

\section{Referencias bibliográficas}

An, Y., Zhu, M., Bonk, C. J. Zhu, M., \& Lin, L. (2021). Exploring instructors' perspectives, practices, and perceived support needs and barriers related to the gamification of MOOCs. Journal of Computing in Higher Education, 33, 64-84. https://doi.org/10.1007/s12528-020-09256-w

Barton, J., Stephens, J., \& Haslett, T. (2009). Action Research: Its Foundations in Open Systems Thinking and Relationship to the Scientific Method. Systemic Practice and Action Research, 22, 475-488.

https://doi.org/10.1007/s11213-009-9148-6

Callejo, J., \& Viedma, A. (2006). Proyectos y estrategias de investigación social: la perspectiva de intervención. McGraw-Hill.

Carson, E. C. (2021). Gamification of Math: Integrating Games into 6th Grade Curriculum to Promote Inquiry-based Learning. Asu Library.

Contreras Espinosa, R. S., \& Eguia, J. L. (2016). Gamificación en aulas universitarias. Institut de la Comunicació, Universitat Autònoma de Barcelona.

Corchuelo, C.A. (2018). Gamificación en educación superior: experiencia innovadora para motivar estudiantes y dinamizar contenidos en el aula. EDUTEC. Revista Electrónica de Tecnología Educativa, 63, 29-41.

https://doi.org/10.21556/edutec.2018.63.927

Chou, Y. (2021). Gamification Examples: the fully comprehensive list. https://bit.ly/3nwDYXX

Deterding, S., Dixon, D., Nacke, L. E., O’Hara, K., \& Sicart, M. (2011). Gamification: Using gamedesign elements in non-gaming contexts. CHI '11. CHI, conference on human factors in computing systems (pp. 2425-2428). ACM. http://dx.doi.org/10.1145/1979742.1979575

Deterding, S., Dixon, D., Khaled, R., \& Nacke, L. (2011). From Game Design Elements to
Gamefulness: Defining Gamification. En Proceedings of the 15th International Academic MindTrek Conference: Envisioning Future Media Environments (pp. 9-15). ACM. http://dx.doi.org/10.1145/2181037.2181040

Domínguez, A., Sáenz-de-Navarrete, J., de Marcos, L., Fernández-Sanz, L., Pagés, C., \& MartínezHerráiz, J. J. (2013). Gamifying learning experiences: Practical implications and outcomes. Computers \& Education, 63, 380-392.

http://dx.doi.org/10.1016/j.compedu.2012.12.020

Fernández-Antolín, M. M., del Río, J. M., \& GonzálezLezcano, R. A. (2021). The use of gamification in higher technical education: perception of university students on innovative teaching materials. International Journal of Technology and Design Education, 31, 1019-1038. https:// doi.org/10.1007/s10798-020-09583-0

Fernández-Gavira, J., Prieto, E., Alcaraz, V., Sánchez-Oliver, A., \& Grimaldi, M. (2018). Aprendizajes significativos mediante la gamificación a partir del juego de rol: "Las Aldeas de la Historia". Espiral. Cuadernos del profesorado, 11(22), 69-78. https://bit.ly/3k993Pj

Gasca-Hurtado, G. P., Gómez-Álvarez, M. C., Muñoz, M., \& Mejía, J. (2017). Toward an assessment framework for gamified environments. In Toward an assessment framework for gamified environments (pp. 281293). (Communications in Computer and Information Science; Vol. 748).

https://doi.org/10.1007/978-3-319-64218-5_23

Gibbs, G. (2012). El análisis de datos cualitativos en Investigación Cualitativa. Ediciones Morata.

González, C. S., Gómez, N., Navarro, V., Cairós, M., Navarro, V., Toledo, P., \& Marrero-Gordillo, N. (2016). Learning healthy lifestyles through active videogames, motor games and the gamification of educational activities. Computers in Human Behavior, 55(A), 529551.

http://dx.doi.org/10.1016/j.chb.2015.08.052

Hamari, J., \& Koivisto, J. (2015). "Working out for likes": An empirical study on social influence in exercise gamification. Computers in Human Behavior, 50, 333-347.

http://dx.doi.org/10.1016/j.chb.2015.04.018

Hossein-Mohand, H., Trujillo-Torres, J. M., GómezGarcía, M., Hossein-Mohand, H., \& Campos- 
Soto, A. (2021). Analysis of the Use and Integration of the Flipped Learning Model, Project-Based Learning, and Gamification Methodologies by Secondary School Mathematics Teachers. Sustainability,13, 2606. https://doi.org/10.3390/su13052606

Huotari, K. \& Hamari, J. (2012). Defining gamification: A service marketing perspective. MindTrek '12. Proceeding of the 16th international academic MindTrek conference (pp. 17-21). ACM. http://dx.doi.org/10.1145/2393132.2393137

Igartua, J. J. \& Humanes, M. L. (2009). Teoría e investigación en Comunicación Social. Síntesis.

Invalsi (2019). Rapporto Prove Invalsi 2019. Rapporto Nazionale. https://bit.ly/3kxffzE

Iñiguez, L. (2006). Análisis del discurso: manual para las ciencias sociales. Editorial UOC.

Johnson, D., Deterding, S., Kuhn, K., Staneva, A., Stoyanov, S., \& Hides, L. (2016). Gamification for health and wellbeing: A systematic review of the literature. Internet Interventions, 6, $89-106$.

http://dx.doi.org/10.1016/j.invent.2016.10.002

Johnson, R., \& Onwuegbuzie, A. J. (2004). Mixed Methods Research: A Research Paradigm Whose Time Has Come. Educational Researcher, 33(7), 14-26.

https://doi.org/10.3102/0013189X033007014

Kapp, K. M. (2012). The gamification of learning and instruction: Game-based methods and strategies for training and education. Pfeiffer.

Kocakoyun, S., \& Ozdamli, F. (2018) A review of research on gamification approach in education. En R. Morese, J. Nervo y S. Palermo (Eds.) Socialization. A multidimensional perspective (pp. 51-73). London: IntechOpen. http://dx.doi.org/10.5772/intechopen.74131

Koivisto, J., \& Hamari, J. (2019). The rise of motivational information systems: A review of gamification research. International Journal of Information Management, 45, 191-210.

http://dx.doi.org/10.1016/j.ijinfomgt.2018.10.013

Landers, R. N., Bauer, K. N., \& Callan, R. C. (2017). Gamification of task performance with leaderboards: A goal setting experiment. Computers in Human Behavior, 71, 508-515. http://dx.doi.org/10.1016/j.chb.2015.08.008

López, P., Rodrigues-Silva, J., \& Alsina, Á. (2021). Brazilian and Spanish Mathematics Teachers'
Predispositions towards Gamification in STEAM Education. Education Sciences, 11(10), 618.

http://dx.doi.org/10.3390/educsci11100618

Pérez-López, I. J., Rivera, E., \& Trigueros, C. (2017). «La profecía de los elegidos»: un ejemplo de gamificación aplicado a la docencia universitaria. Revista Internacional de Medicina y Ciencias de la Actividad Física y el Deporte, 17(66), 243-260.

https://doi.org/10.15366/rimcafd2017.66.003

Pisabarro, A.M., \& Vivaracho, C.E. (2018). Gamificación en el aula: gincana de programación. ReVisión, 11(1), 85-93.

https://bit.ly/3lsrQog

Qahri-Saremi, H., \& Turel, O. (2016). School engagement, information technology use, and educational development: An empirical investigation of adolescents. Computers \& Education, 102, 65-78.

http://dx.doi.org/10.1016/j.compedu.2016.07.004

Quintero, L., Jiménez, F., \& Area, M. (2018). Más allá del libro de texto. La gamificación mediada con TIC como alternativa de innovación en Educación Física. Retos, 34, 343-348.

https://doi.org/10.47197/retos.v0i34.65514

Ramírez, J. L. (2014). Gamificación. Mecánicas de juegos en tu vida personal y profesional. RC Libros.

Rule, P., \& Mitchell, J. (2015). A Necessary Dialogue: Theory in Case Study Research. International Journal of Qualitative Methods, 1-11.

https://doi.org/10.1177/1609406915611575

Santos-Ferreira, B., \& Lacerda-Santos, G. (2018). Gamificación como estrategia didáctica. Aplicación en la formación del profesor. Tendencias Pedagógicas, 31, 113-126.

https://doi.org/10.15366/tp2018.31.006

Sardi, L., Idri, A., \& Fernández-Alemán, J. L. (2017). A systematic review of gamification in e-health. Journal of Biomedical Informatics, 71, 31-48. http://dx.doi.org/10.1016/j.jbi.2017.05.011

Seaborn, K., \& Fels, D. I. (2015). Gamification in theory and action. International Journal of Human-Computer Studies, 74(C), 14-31. http://dx.doi.org/10.1016/j.ijhcs.2014.09.006

Stake, R. E. (2007). Investigación con estudio de casos. Ediciones Morata 
Stake, R. E. (2005). Qualitative case studies. En N. Denzin, y Y. Lincoln: The SAGE Handbook of Qualitative Research, 443-466. SAGE Publications.

Tomislav, J., Ivica, B., \& Hyo-Jeong, S. (2018). Examining competitive, collaborative and adaptive gamification in young learners' math learning. Computers \& Education, 125, 444-457.

https://doi.org/10.1016/j.compedu.2018.06.022

Torres-Toukoumidis, A., Romero-Rodríguez, L. M., Pérez-Rodríguez, M. A., \& Björk, S. (2018). Modelo Teórico Integrado de Gamificación en Ambientes E-Learning (E-MIGA). Revista Complutense de Educación, 29(1), 129-145. https://doi.org/10.5209/RCED.52117

Villagra-Arnedo, C., Gallego-Durán, F. J., MolinaCarmona, R., \& Llorens-Largo, F. (2016). PLMan: Towards a gamified learning system. En P. Zaphiris y A. Ioannou (Eds.), Learning and collaboration technologies (pp. 82-93). Springer International Publishing.

http://dx.doi.org/10.1007/978-3-319-39483-1_8

Valles, M. (2000). Técnicas cualitativas de investigación social. Síntesis.
Van Dijk, T. A. (2005). Ideología y análisis del discurso [Ideology and discourse analysis]. Utopía y Praxis Latinoamericana, 10(29), 9-36.

https://bit.ly/3zzq2Q4

Werbach, K., \& Hunter, D. (2012). For the win: How game thinking can revolutionize your business. Wharton Digital Press.

Widodo, S., \& Rahayu, P. (2019). Analysis of elementary school students' mastery in math instruction based on arithmetic gamification. Journal of Physics: Conference Series, 1157(4), 012112. IOP Publishing.

Zaharin, F. Z., Abd Karim, N. S., Adenan, N. H., Md Junus, N. W., Tarmizi, R. A., Abd Hamid, N. Z., \& Abd Latib, L. (2021). Gamification in Mathematics: Students' Perceptions in Learning Perimeter and Area. Jurnal Pendidikan Sains Dan Matematik Malaysia, 11, 72-80.

https://doi.org/10.37134/jpsmm.vol11.sp.7.2021

Zichermann, G., \& Cunningham, C. (2011). Gamification by design: Implementing game mechanics in web and mobile apps. O'Reilly.

Zichermann, G., \& Linder, J. (2013). Game-based marketing: Inspire customer loyalty through rewards, challenges, and contests. Wiley. 


\title{
EITERIDAD Una app móvil potencia la motivación del alumnado (36) 01 en una experiencia de gamificación universitaria
}

\author{
A phone app as an enhancer of students' motivation in a \\ gamification experience in a university context
}

D Carmen Navarro-Mateos es estudiante de Doctorado en la Universidad de Granada (España) (carmenavarro@correo.ugr.es) (https://orcid.org/0000-0002-0757-7975)

D Dr. Isaac J. Pérez-López es profesor e investigador de la Universidad de Granada (España) (isaacj@ugr.es) (https://orcid. org/0000-0002-4156-7762)

Recibido: 2021-11-03 / Revisado: 2021-12-08 / Aceptado: 2021-12-14 / Publicado: 2022-01-01

\section{Resumen}

Los niveles de desinterés y desmotivación de gran parte del alumnado universitario representan uno de los retos más urgentes que debe afrontar el profesorado en la actualidad. En este sentido, cada vez son más los trabajos que evidencian el potencial de la "gamificación" a la hora de incrementar la motivación e implicación de los estudiantes en su proceso formativo. En estrecha relación con esto, las tecnologías digitales y, concretamente, el uso de dispositivos móviles supone un recurso de enorme significatividad para jóvenes y adolescentes. Por tanto, en este trabajo se describe la aplicación "\$in TIME", diseñada "ad hoc" con la intención de gestionar los diferentes elementos que conformaron un proyecto de "gamificación" basado en la película "In Time". Este se implementó en la asignatura Fundamentos de la Educación Física (del segundo curso del grado en Ciencias de la Actividad Física y el Deporte, de la Universidad de Granada-España-). De igual modo, también se muestra la repercusión que tuvo en el alumnado participante, así como la valoración que hicieron de la misma. Todo ello permitirá a los docentes conocer un ejemplo real, que puede servir de ayuda e inspiración a la hora de diseñar sus futuros proyectos y, al mismo tiempo, contar con un nuevo aval del potencial de la "gamificación" en el ámbito educativo.

Descriptores: Educación física, universidad, motivación, aprendizaje activo, tecnología educativa.

\section{Abstract}

The level of disinterest and lack of motivation in great part of the university student body represent one of the most urgent challenges that the faculty must face nowadays. In this way, there are more and more projects that show the potential of gamification when it comes to increasing motivation and implication of students in their learning process. In close relationship, digital technologies and, specifically, the use of mobile phone devices entail a resource of enormous meaning for young adults and teenagers. Therefore, in the present project we describe the application "\$in TIME", designed ad hoc with the intention to manage the different elements that defined a gamification project based on the movie "In Time". This was implemented in the course Basis of Physical Education (a second-year course in the BSc in Science of the Physical Activity and Sports in Granada -Spain-). In the same way, it also shows the repercussion that the participating students had, as well the evaluation they did of it. All of this will allow teaching staff to know a real example, which could help them and inspire them when it comes to designing their future projects and, at the same time, they will count with new evidence of the potential of gamification in education.

Keywords: Physical education, university, motivation, experiential learning, educational technology.

Forma sugerida de citar: Navarro-Mateos, C., \& Pérez-López, I. J. (2022). Una app móvil potencia la motivación del alumnado en una experiencia de gamificación en contexto universitario. Alteridad, 17(1), 64-74. https://doi.org/10.17163/alt. 


\section{Introducción}

Uno de los principales problemas a los que se enfrenta la educación universitaria es la falta de motivación del alumnado, que se traduce en una falta de compromiso e implicación con su proceso formativo (Symonds et al., 2019; Zhao \& Hoge, 2005). Ante esta alarmante realidad son necesarios planteamientos que sitúen al alumnado en el centro del proceso de enseñanza y aprendizaje para romper con la unidireccionalidad que caracteriza al enfoque tradicional y poder dotarles de la capacidad de decisión para lograr un aprendizaje significativo (Gargallo-López et al., 2017; Ituma, 2011). Es en este contexto donde surgen metodologías activas que pretenden convertir al alumno en el protagonista de su proceso formativo, acercando la realidad del siglo XXI a las aulas (McLean \& Gibbs, 2010; Crisol-Moya et al., 2020). La evidencia científica nos muestra cómo, a través de ellas, se potencia la resolución de problemas, el pensamiento crítico, la capacidad de análisis y otras habilidades cognitivas complejas relacionadas con las competencias (Martínez-Hita \& Gómez, 2018; Sáiz, 2013).

Siguiendo con la búsqueda de posibles soluciones para aumentar la significatividad del alumnado, una poderosa estrategia para motivar y favorecer el aprendizaje es la "gamificación" (Deterding et al., 2011), entendida como el uso de mecánicas y dinámicas de los juegos en contextos no lúdicos, ya que, a través de ella, el alumnado puede incrementar su interés y compromiso hacia la propuesta planteada por el docente (Kapp, 2012; Zichermann \& Cunningham, 2011). En línea con ello, las películas y series de televisión se han convertido en la actualidad en una de las principales y más importantes fuentes de ocio de jóvenes y adolescentes (Medina \& González, 2013; Scolari et al., 2019). Es en este contexto en el que Pérez-López (2018) establece el concepto de "gamificción", es decir, aprovechar en el ámbito educativo los aspectos claves de una referencia fílmica para aumentar la motivación del alumnado, gracias al atractivo y significatividad que les supone vivir en primera persona aquello que previamente han estado disfrutando como espectadores. Para ello, se deben tener en cuenta los seis aspectos clave que Pérez-López \& Navarro-Mateos (2019) establecen en su "Tetris de la gamificción": tiempo, estética, tramas, roles y personajes e interacciones. De ese modo se favorecerá la credibilidad de la propuesta, haciendo que la "inmersión" del alumnado sea mayor.

Si a todo ello se le suma el uso de dispositivos móviles (que los/las jóvenes utilizan a diario) como herramientas educativas, su motivación puede incrementar aún más (Brazuelo \& Gallego, 2014; Mojarro et al., 2015; RamírezMontoya \& García-Peñalvo, 2017). De hecho, en los últimos años, la implementación de las tecnologías digitales en las distintas etapas educativas ha aumentado notoriamente debido a sus beneficios en el desarrollo del pensamiento creativo, la resolución de problemas y el aumento de la eficiencia y productividad, lo que se traduce en una mejora de los resultados académicos (Adeosun, 2010; Fullan et al., 2018; McMahon, 2009). La universidad no puede permanecer ajena a este desarrollo tecnológico, cambiando las estrategias y actividades que se plantean para adaptarse a las nuevas oportunidades que surgen (Almerich et al., 2021; Fatykhova et al., 2018).

En esta etapa es clave desarrollar las competencias necesarias para desempeñar una buena labor profesional (Shavelson et al., 2019). Esto convierte a la universidad en el escenario perfecto para el desarrollo de la competencia digital, tan demandada por la sociedad actual, percibiendo el alumnado que el uso de las tecnologías digitales es beneficioso para su aprendizaje y para el desarrollo de competencias fundamentales para el mañana (Karakoyun \& Lindberg, 2020). Es por ello que en una sociedad que se desarrolla rápidamente debido al aumento y desarrollo de las tecnologías, el ámbito educativo debe preocuparse por crear contextos en los que el alumnado pueda adquirir competencias que les permitan analizar críticamente la información 
que reciben y adaptarse a los desarrollos tecnológicos de nuestra era (Baranowski \& OdrowążCoates, 2018; Fatykhova et al., 2018; Olszewski \& Crompton, 2020; Schmidt et al., 2020).

El objetivo de este artículo es, por tanto, describir una aplicación móvil diseñada "ad hoc" para el proyecto de "gamificción" "\$in Time” y conocer las percepciones del alumnado, así como la incidencia que esta tuvo en la motivación.

\section{Contextualización}

El proyecto de "gamificción" denominado "\$in Time" se llevó a cabo en la asignatura Fundamentos de la Educación Física, desarrollada durante el segundo semestre del segundo curso del Grado de Ciencias de la Actividad Física y el Deporte (Universidad de Granada). Esta asignatura es de carácter obligatorio con una carga lectiva de seis créditos. Los principales objetivos de la misma se muestran a continuación:

- Saber planificar la Educación Física (EF) en la etapa de secundaria siguiendo los principios de jerarquización vertical y horizontal.

- Conocer, saber diseñar y manejar los elementos principales del currículo: objetivos, contenidos y temporalización.

- Planificar un curso escolar con unidades didácticas según sus objetivos educativos.

- Revisar y analizar informes de innovaciones y experiencias en la EF, aplicándolos a la planificación de la EF.

Entre los contenidos destacan:

- Fundamentos de la Educación. Educación Física.

- El papel del profesor en el marco educativo y la sociedad actual. Competencias fundamentales.

- El deporte y la actividad física como vehículo de formación general del individuo en la sociedad. Intervenir para educar en valores.

- Fundamentos de la planificación en Educación Física.

- La innovación: el motor del conocimiento práctico en Educación.

\section{Narrativa}

La ambientación de la propuesta se basa en la película "In Time", de Andrew Niccol. En 2117 la evolución de la humanidad ha llegado a tal punto que ha logrado desactivar el gen del envejecimiento a partir de los 18 años. Pero, como contrapartida, desde ese momento solamente cuentan con un año más de vida, salvo que trabajen para acumular o recuperar algo más de tiempo.

En esta experiencia de aprendizaje denominada "\$in TIME" (cuyo logo se puede apreciar en la figura 1) el "Metronomista" es el dueño del banco del tiempo, condicionando la vida de los habitantes de la ciudad. Él es quien determina sus sueldos, los impuestos, los intereses de cualquier préstamo, etc., modificándolos a su antojo. Su principal finalidad es que los ricos cada día sean más ricos y los pobres más pobres. En este sentido, para tener controlados a los ciudadanos del "gueto de Feni" (el alumnado), se vale de los "Minuteros", una banda de matones a su servicio que sobreviven robándole tiempo a la gente común para no tener que trabajar ellos. Por tanto, la idea sobre la que se construye la propuesta es que la vida es tiempo y el tiempo es una oportunidad para enriquecer la vida a través del aprendizaje (para ganar vida, disfrutando con ello) y, a su vez, su "moneda" de cambio, con la que poder comprar, por ejemplo, comida (representada por la realización de retos formativos) y agua (retos creativos). En función de cómo se aproveche ese tiempo el alumnado podría aumentar su tiempo de vida. 


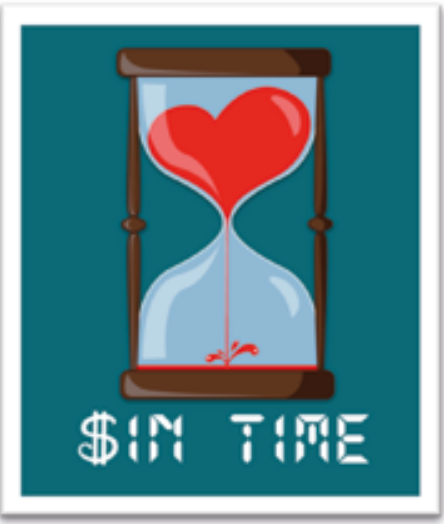

\section{Papel de las tecnologías digitales}

En la película la gente tenía un contador de tiempo en el antebrazo que iba marcando el tiempo de vida que les quedaba. En el proyecto lo llevaban en su teléfono móvil, dado que como complemento al proyecto se desarrolló una aplicación web para el móvil desde la cual se gestionó toda la asignatura (figura 2).

Gracias a la app se pudieron recrear muchas de las emociones que experimentaban los personajes de la película, teniendo que planificarse —uno de los objetivos de la asignatura- del mejor modo posible para no quedarse sin tiempo y "fallecer" (suspender). De hecho, su primer objetivo dentro de la aventura era evitar que su contador de tiempo llegara a cero, lo que les permitiría convertirse en "Troyanos de la Educación”, objetivo final del proyecto. Al mismo tiempo, tenían que tratar de localizar al "Metronomista" para recuperar todo el tiempo que él les robaba y poder repartirlo entre el resto de habitantes de la ciudad para, de este modo, dejar de vivir con la presión del tiempo permanentemente.

Figura 2. Pantalla de inicio y menú principal de la app "Sin TIME"
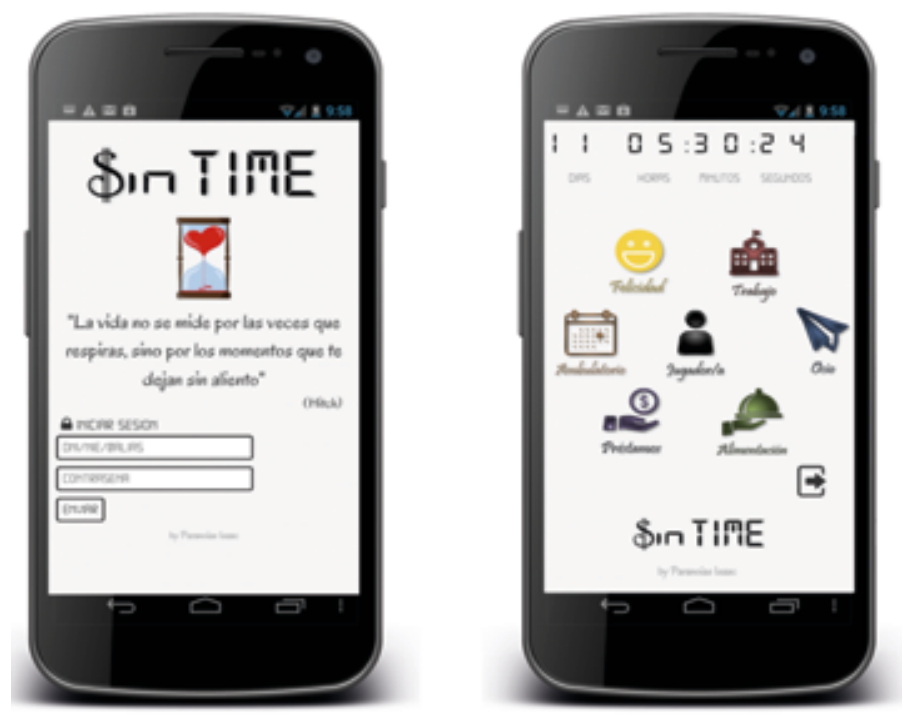
La app permitió gestionar diferentes actividades habituales en la vida de una persona, $o$ un futuro docente en este caso, divididas en seis grandes categorías con sus correspondientes subapartados. A continuación, se describirá cada una de ellas, lo que permitirá conocer su funcionamiento y potencialidad:

\section{Trabajo}

- Jornada laboral: a través de "Twitter" el alumnado podía realizar un seguimiento de las cuentas de personas relevantes en el ámbito de la EF, y la Educación en general, con la intención de aprovechar el enorme aprendizaje que puede proporcionar una red social como esta y, al mismo tiempo, compartir lo vivido y aprendido, aspectos fundamentales en un futuro docente. El uso de "Twitter" ayuda a predisponer positivamente al alumnado y compartir a través de esta red de "microblogging" fragmentos de su vida, comunicarse con otros compañeros y expertos en determinados campos, fomentar la colaboración, organizar la clase, reflexionar y evaluar (Tang \& Hew, 2017).

- Inspección de trabajo: el "Guardián del Tiempo" (docente de la asignatura) mandaba una pregunta cada cierto tiempo (que les llegaba a modo de mensaje/aviso) y tenían que contestarla lo antes posible entrando en la sección correspondiente de la app. Tras las seis primeras respuestas correctas la app indicaba que el tiempo de respuesta había finalizado, y aquellos que habían respondido a tiempo aumentarían su tiempo de vida.

- Paga extra: el "Guardián del Tiempo" proponía un reto y quienes quisieran aceptarlo debían entrar en la sección correspondiente de la app y marcar OK. Tras las seis primeras respuestas afirmativas se cerraba la posibilidad de acceder a esta bonificación de tiempo de vida que lograban quienes lo resolvían adecuadamente.

- Proyecto de innovación: esta sección era en la que los "distritos" (grupos) debían subir su proyecto de innovación (figura 3), donde se recogía todo el aprendizaje adquirido durante la aventura, que posteriormente tenían que defender ante el "Clan de los Temporizadores" (antiguos alumnos y alumnas que habían cursado el Máster de Profesorado), quienes determinaban, junto al "Guardián del Tiempo", la calidad de los mismos y el correspondiente incremento de su tiempo de vida.
(C) to emartaboutista 3 - 31 mas. 2017

Gracias por acompañarme y enseñarme tanto a lo largo del camino. La unión hace la fuerza. \#ProyectosinTime Hooposcanarios HProyectoRishe/

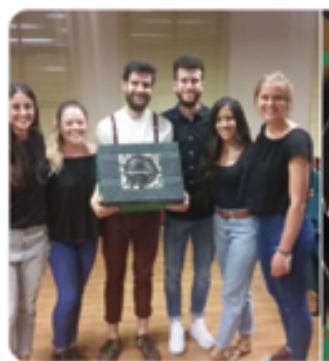
( $\mathrm{ta} 4$

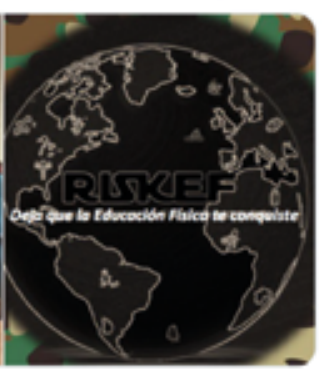

$\bigcirc 5$
4

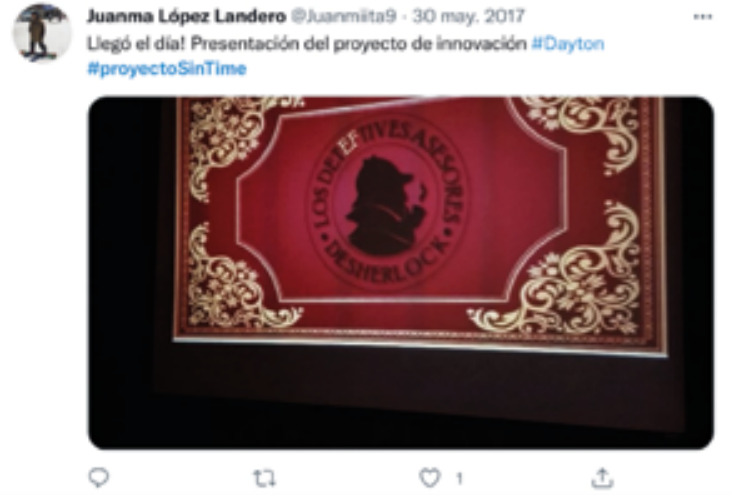




\section{Ocio}

- Deporte: esta sección estaba conectada con la aplicación "Runtastic" y permitía a los jugadores (alumnado) descargar las sesiones que iban realizando, corriendo o montando en bicicleta, y obtener bonificaciones de tiempo por ello.

- Apuestas: tenían la posibilidad de apostar tiempo de vida en las diferentes actividades grupales que se hacían en sesiones prácticas, y que consistían en retos físicodeportivos.

- Vacaciones: existía la posibilidad de conseguir una carta que permitía detener el contador de tiempo durante una semana.

- Altruismo

- Donar: se les daba la posibilidad de dar tiempo a otros compañeros cuyo contador de tiempo se acercaba a 0 .

- Minas: consistía en localizar una "mina" (código QR) por la ciudad antes de un tiempo determinado para evitar que todos redujeran su tiempo de vida. Tenían la posibilidad de comprar una pista a cambio de tiempo o comprando la carta correspondiente. "si lo lograban," la bonificación de tiempo de vida se dividía entre el número de jugadores que la hubieran localizado durante el tiempo establecido.

- Amigos: podían comunicarse con el resto de compañeros a través de un chat sin salir de la app, o subir las fotos de lo realizado en las diferentes sesiones presenciales que tenían, o de aquellos momentos relevantes fuera de ellas que quisieran compartir con el resto de ciudadanos del "gueto de Feni" y con el "Guardián del Tiempo".

\section{Alimentación}

Aquí se establece un paralelismo entre comer con la realización de retos formativos y la necesidad de hidratarse con el planteamiento de retos creativos, donde como si estuvieran en un supermercado podían comprar el alimento/reto que consideraran más oportuno en cada momento (favoreciendo así el ritmo de aprendizaje de cada cual), y tras su resolución obtener un mayor tiempo de vida en función de la calidad del mismo.

\section{Préstamos}

Los jugadores podían enviar una solicitud de préstamo al "Metronomista" con la cantidad de tiempo solicitada y el número de plazos de devolución. En función de la cantidad de tiempo de vida solicitada la contrapartida — intereses - era diferente. El tiempo de vida aumentaba tras el visto bueno del "Metronomista", pero en caso de no cumplir con la contrapartida adecuadamente en el plazo establecido se incrementaba la penalización.

\section{Ambulatorio}

En este apartado tenían un calendario para poder solicitar citas con el "Guardián del Tiempo" y resolver, por ejemplo, cualquier tipo de duda relacionada con los diferentes retos que debían superar en la aventura. Un aspecto de gran relevancia, y muy valorado por el alumnado participante, es que no tenían una única posibilidad de entrega de los retos que decidían afrontar. Conforme iban aprendiendo y mejorando su formación podían tratar de resolver nuevamente cualquiera de los ya realizados previamente fomentando, entre otras cosas, su creatividad. Además, estas citan tenían costes en tiempo de vida, lo que aumentaba su valor y aprovechamiento, dotando del valor que se merece al tiempo invertido en aspectos formativos.

\section{Felicidad}

Con esta sección se le daba la oportunidad al alumnado de hacer un poco más felices a las personas de su entorno (familiares y amigos). Para ello se comprometían a hacer "PDFs" (Proyectos de Felicidad) de manera altruista, con toda la 
planificación y dedicación que ello requería (Pérez-López, 2019).

Además, en la aplicación existe también un apartado de "Jugador/a" donde se incluye una clasificación individual y por "distritos" (grupos), el nivel de cada participante en función de los logros y evidencias que mostraban de su aprendizaje o los puntos de experiencia. Estos últimos podían ser canjeados por diferentes cartas de privilegios para hacerles la vida más llevadera dentro de la aventura como, por ejemplo:

- La carta "libre de minuteros", gracias a la cual no era necesario tener que "huir" de ellos durante una semana, ya que durante ese tiempo no podrían "robarle" tiempo.

- La carta de "beneficio doble", que permitía a quien la tuviera doblar el tiempo obtenido en el último reto realizado.

\section{Análisis e interpretación de las percepciones del alumnado}

Para conocer las sensaciones, aprendizajes y reflexiones del alumnado se habilitó un enlace a "Google Drive" en el que podían compartir de manera anónima todo aquello que les apeteciera. Además, muchos de ellos hicieron referencia al proyecto en sus cuentas personales en redes sociales. A continuación, se ponen algunos ejemplos que compartieron en "Twitter" sobre lo que significó para ellos esta experiencia de aprendizaje, a modo resumen:

- "Una manera real de aprender. Gracias \#Isaac por todo lo que nos has hecho vivir \#ProyectoSinTime".

- “¡Porque esto es solo el inicio! ¡Troyano de la educación con muchísimo por aprender y con ganas de hacerlo! \#ProyectoSinTime".

- “¡El fin se encuentra al inicio! ¡Increíble experiencia de aprendizaje la vivida este cuatrimestre, esto sí es educación! \#ProyectoSinTime".

Por otro lado, en los fragmentos que se comparten seguidamente se puede apreciar la motivación que al alumnado le supuso tener una aplicación creada "ad hoc" para el proyecto, facilitándoles además el seguimiento de la aventura, tal y como muestra Julio (todos los nombres utilizados son ficticios, ya que la procedencia de la información fue anónima):

Cada día me motivo más con la aplicación. Es algo que jamás me hubiera esperado de un profesor, que diseñara algo así para sus alumnos. Un ejemplo es que nunca me ha gustado salir a correr, pero hoy lo he hecho motivado por la app y me ha encantado, además he podido disfrutar de miradores preciosos. Otro aspecto a resaltar es la gran cantidad de secciones que incluye. Podemos gestionar todo lo relacionado con la asignatura desde ella, me parece increíble, ¡una pasada!

En esta misma línea se sitúa el testimonio de Lucía:

La aplicación me parece alucinante, cuidando hasta el más mínimo detalle. Ya era hora de que algún profesor me hiciera ver que existe otra forma de hacer las cosas y lo más importante, que tenga ganas de volver a clase en cuanto salgo de ella. Cada clase se me pasa volando. Gracias por hacerme ver y reflexionar sobre cómo se pueden hacer las cosas diferentes. Además, entre el contador de tiempo y el historial de todos nuestros movimientos durante la aventura nunca había tenido tanto "feedback" ni tan detallado en una asignatura. ¡Una pasada!

Uno de los grandes retos de todo docente es lograr que el alumnado esté permanentemente conectado a la asignatura (más allá de las horas presenciales de clase). El hecho de aprovechar aquello que es significativo para el alumnado, como puede ser el uso de los dispositivos móviles, puede aumentar su predisposición y motiva- 
ción hacia la misma. De hecho, buena muestra de ello es la reflexión de Pedro:

Reconozco que estás consiguiendo que le dedique muchísimo más tiempo a esta asignatura que a ninguna otra, pero es que gracias a la app has logrado que estemos permanentemente conectados con la asignatura. Además, tiene tanta variedad de secciones y muchas de ellas tan habituales a las que solemos utilizar que ya casi la uso más que "WhatsApp" o "Instagram". ¡Me lo dicen y no me lo creo, jajajaja!

Esta sensación también es compartida por Rosa:

¡Estoy ansiosa por tener ya la app en mi móvil! Es increíble el trabajazo que tiene, pero más increíble es que TODOS estemos deseando ponernos a trabajar y estemos enganchadísimos a la asignatura. Personalmente, es la primera vez que voy a llevar a cabo de esta manera una asignatura y me tiene motivadísima. ¡Desde ya te digo que me esforzaré todo lo que pueda!

Estos resultados van en la línea de otras investigaciones en las que se usaban diferentes aplicaciones y dispositivos móviles para mejorar la motivación y calidad de los aprendizajes en diferentes carreras universitarias (Pechenkina et al., 2017; Weibers et al., 2019).

Como se indicaba al comienzo, en "gamificción” es clave recrear las sensaciones y emociones de la referencia fílmica en cuestión para aumentar la "inmersión" y credibilidad (PérezLópez \& Navarro-Mateos, 2019). Tal y como expresa Fernando, gracias al contador de tiempo que incluía la aplicación, los alumnos vivieron emociones similares a las de los protagonistas de "In Time":

¡Menuda tensión me está generando la aplicación! Si querías que tuviéramos que gestionar emociones lo has conseguido, y a base de bien. Estás logrando que aproveche el tiempo como nunca antes lo había hecho, ya me planifico hasta el más mínimo detalle de mi día a día. Si se lo contara a mis padres ni se lo creerían.

Con todo ello se puede comprobar la gran acogida que tuvo la app móvil dentro del proyecto. Es necesario destacar que, además, el grupo que participó en la propuesta de "gamificción" mejoró de manera muy significativa su fitness cardiorrespiratorio, parámetro de gran relevancia en la salud del alumnado, en comparación con el grupo que siguió una metodología de aprendizaje tradicional (Mora-González et al., 2020). En relación con ello, cada vez se encuentran más intervenciones en la literatura científica que aprovechan los dispositivos móviles y el uso de elementos de los juegos para aumentar los niveles de actividad física (Cotton \& Pattel, 2019) y la salud y calidad de vida de diferentes poblaciones, como pacientes de avanzada edad o con patologías como la hipertensión, la obesidad o el autismo (Cechetti et al., 2019; Kappen et al., 2016; Lee et al., 2018; Mâsse et al., 2020; Ryan et al., 2017).

\section{Conclusiones}

Tras todo lo expuesto se hace evidente las posibilidades que ofrece el uso de las tecnologías y, en concreto, de los dispositivos móviles en el ámbito educativo enriqueciendo, en este caso, una experiencia de "gamificción". Al incluir aspectos de enorme significatividad para el alumnado se incide sobre su motivación e implicación, lo que conlleva a un incremento del aprendizaje. Gracias a la aplicación que se diseñó para este proyecto, el alumnado tuvo la oportunidad de estar continuamente conectado a la asignatura, trascendiendo más allá del tiempo de clase. Además, mediante ella se podían gestionar todos los aspectos relativos al proyecto y, en concreto, a la aventura que estaban viviendo (solicitar tutorías, entregar retos, recibir "feedback", hacer consultas al resto de compañeros, etc.).

Por último, destacar la importancia de generar en el alumnado sensaciones y emociones similares a las que viven los protagonistas de 
la referencia fílmica en cuestión en este tipo de proyectos, puesto que de este modo se aumentará la "inmersión", favoreciendo la implicación y compromiso de estos con su formación. En este caso se recreó la principal emoción que vivían los protagonistas de la película "In Time", esa continua presión del tiempo y el necesario aprovechamiento del mismo para poder seguir con vida. El alumnado debía gestionar muchas emociones y planificar adecuadamente su tiempo para poder disfrutar al máximo la experiencia. Todos esos aprendizajes, al vincularlos a diferentes emociones, tal y como expresa la neuroeducación (Mora, 2017), tienen un mayor arraigo.

De cara a las futuras líneas de trabajo, sería interesante seguir analizando el potencial de la narrativa y de los elementos estéticos en aplicaciones que pretenden incidir sobre variables relacionadas con la salud, y donde la adherencia es un factor fundamental. Al construir una experiencia "inmersiva”, que va más allá de elementos extrínsecos como los puntos o clasificaciones, la motivación y las diferentes sensaciones vividas aumentarán ese compromiso con la propuesta que favorecerá la adquisición de los objetivos planteados.

\section{Referencias bibliográficas}

Adeosun, O. (2010). Quality basic education development in Nigeria: Imperative for use of ICT. Journal of International Cooperation in Education, 13(2), 193-211. https://bit. ly/3pWv5Xg

Almerich, G., Suárez-Rodríguez, J., Díaz-García, I., \& Orellana, N. (2021). The influence of using ICT in high-skills competences and ICT competences. A structural model. Education and Information Technologies, 26, 3845-3869. https://doi.org/10.1007/s10639-021-10437-y

Baranowski, M., \& Odrowąż-Coates, A. (2018). Attempting to register changes: the educational dimention of contemporary societies. Society Register, 2(2), 7-10. https://doi. org/10.14746/sr.2018.2.2.01

Brazuelo, F., \& Gallego, D.J. (2014). Estado del mobile learning en España. Educar em revista, 4, 99-128. https://doi.org/10.1590/0104-4060.38646

Cechetti, N. P., Bellei, E. A., Biduski, D., Mazuco, J. P., Klein, M., \& Bertoletti De Marchi, A. C. (2019). Developing and implementing a gamification method to improve user engagement: A case study with an m-Health application for hypertension monitoring. Telematics and Informatics, 41,126-138. https://doi.org/10.1016/j.tele.2019.04.007

Cotton, V., \& Patel, M. S. (2019). Gamification use and design in popular health and fitness mobile applications. American Journal of Health Promotion, 33(3), 448-451.

https://doi.org/10.1177/0890117118790394

Crisol-Moya, E., Romero-López, M. A., \& CaurcelCara, M. J. (2020). Active Methodologies in Higher Education: Perception and Opinion as Evaluated by Professors and Their Students in the Teaching-Learning Process. Frontiers in Psychology, 11, 1703.

https://doi.org/10.3389/fpsyg.2020.01703

Deterding, S., Sicart, M., Nacke, L., O'Hara, K., \& Dixon, D. (2011). Gamification. using gamedesign elements in non-gaming contexts. In CHI'11 extended abstracts on human factors in computing systems (pp. 2425-2428).

Fatykhova, A., Rakhmanina, M., Vasilenko, P., Vasilenko, E., \& Gere v, E. (2018). Development of corporate standard model of ict-competence of academic staff of a modern university. International Journal of Civil Engineering and Technology, 9(13), 1027-1034. https://bit.ly/3EOsEMK

Fullan, M., Quinn, J., \& McEachen, J. (2018). Deep learning. Engage the world change the world. ThousandOaks, California, EEUU: Corwin.

Gargallo-López, B., Pérez-Pérez, C., Verde-Peleato, I., \& García-Félix, E. (2017). Estilos de aprendizaje en estudiantes universitarios y enseñanza centrada en el aprendizaje. RELIEVE-Revista Electrónica De Investigación y Evaluación Educativa, 23(2).

https://doi.org/10.7203/relieve.23.2.9078

Ituma A. (2011). An evaluation of students' perceptions and engagement with e-learning components in a campus based university. Active. Learning in Higher Education, 12(1), 57-68. https://doi.org/10.1177/1469787410387722 
Kapp, K. M. (2012). The Gamification of Learning and Instruction: Game-based Methods and Strategies for Training and Education. John Wiley \& Sons.

Kappen, D. L., Nacke, L. E., Gerling, K. M., \& Tsotsos, L. E. (2016). Design strategies for gamified physical activity applications for older adults. En 49th Hawaii International Conference on System Sciences (HICSS) (pp. 1309-1318). IEEE.

Karakoyun, F., \& Lindberg, O. J. (2020). Preservice teachers'views about the twenty-first century skills: Aqualitative survey study in Turkey and Sweden. Education and Information Technologies, 25(4), 2353-2369. https://doi. org/10.1007/s10639-020-10148-w.

Lee, D., Frey, G., Cheng, A., \& Shih, P. C. (2018). Puzzle walk: A gamified mobile app to increase physical activity in adults with autism spectrum disorder. En 2018 10th International Conference on Virtual Worlds and Games for Serious Applications (VS-Games) (pp. 1-4). IEEE.

Martínez-Hita M., \& Gómez C. J. (2018). Nivel cognitivo y competencias de pensamiento histórico en los libros de texto de Historia de España e Inglaterra. Un estudio comparativo. Revista de Educación, 379,145-169. https:// bit.ly/31Z6VTE

Mâsse, L. C., Vlaar, J., Macdonald, J., Bradbury, J., Warshawski, T., Buckler, E. J., ... \& Ball, G.D. (2020). Aim2Be mHealth intervention for children with overweight and obesity: study protocol for a randomized controlled trial. Trials, 21(1), 1-14. https://doi. org/10.1186/s13063-020-4080-2

McLean, M., \& Gibbs, T. (2010). Twelve tips to designing and implementing a learner-centred curriculum: Prevention is better than cure. Medical teacher, 32(3), 225-230.

https://doi.org/10.3109/01421591003621663

McMahon, G. (2009). Critical thinking and ICT integration in a Western Australian secondary school. Educational Technology \& Society, 12(4), 269-281. https://bit.ly/3J0vDEu

Medina, E., \& González, P. (2013). Los jóvenes españoles demandan una televisión con más ficción y entretenimiento. Ámbitos. Revista internacional de Comunicación, 22, 1-11. https://bit.ly/3s6D72s

Mojarro, Á., Rodrigo-Cano, D., \& Etchegaray, M. C. (2015). Educación personalizada a través de e-Learning. Alteridad, 10(1), 21-30.

https://doi.org/10.17163/alt.v10n1.2015.02

Mora. F. (2017). Neuroeducación. Solo se puede aprender aquello que se ama. Alianza Editorial.

Mora-González, J., Pérez-López, I. J., \& DelgadoFernández, M. (2020). The “\$in TIME” gamification project: using a mobile app to improve cardiorespiratory fitness levels of college students. Games Health Journal, 9(1), 37-44. https://doi.org/10.1089/g4h.2019.0001

Olszewski, B., \& Crompton, H. (2020). Educational technology conditions to support the development of digital age skills. Computers \& Education, 150(2).

https://doi.org/10.1016/j.compedu.2020.103849

Pechenkina, E., Laurence, D., Oates, G., Eldridge, D., \& Hunter, D. (2017). Using a gamified mobile app to increase student engagement, retention and academic achievement. International Journal of Educational Technology in Higher Education, 14(1), 1-12. https://doi.org/10.1186/s41239-0170069-7

Pérez-López, I. J. (2018). La docencia es un juego donde gana el que más disfruta. Habilidad motriz, 50, 2-3. https://bit.ly/3e7p7O5

Pérez-López, I. J. (2019). "Proyectos de felicidad": El aprendizaje a través de la experiencia en Educación Superior. En Inclusión, Tecnología y Sociedad: investigación e innovación en educación (pp. 1024-1038). Dykinson.

Pérez-López, I. J., \& Navarro-Mateos, C. (2019). Gamificción: qué, cómo y por qué. Un relato basado en hechos reales. En $15^{\circ}$ Congreso Internacional de Ciencias del Deporte y la Salud (pp. 108-119). Sportis.

Ramírez-Montoya, M. S., \& García-Peñalvo, F. J. (2017). La integración efectiva del dispositivo móvil en la educación y en el aprendizaje. Revista Iberoamericana de Educación a Distancia, 20(2), 29-47. https://bit.ly/3saLytJ

Ryan, J., Edney, S., \& Maher, C. (2017). Engagement, compliance and retention with a gamified online social networking physical activity 
intervention. Translational behavioral medicine, 7(4), 702-708.

https://doi.org/10.1007/s13142-017-0499-8

Sáiz, J. (2013). Alfabetización histórica y competencias básicas en libros de texto de historia y en aprendizajes de estudiantes. Didáctica de las Ciencias Experimentales y Sociales, 27, 43-66. https://bit.ly/33kZOpm

Schmidt, S., Zlatkin-Troitschanskaia, O., Roeper, J., Klose, V., Weber, M., Bültmann, A. K., \& Brückner, S. (2020). Undergraduate students'critical online reasoning-Process mining analysis. Frontiers in Psychology, 11. https://doi.org/10.3389/fpsyg.2020.576273

Scolari, C. A., Lugo-Rodríguez, N., \& Masanet, M. J. (2019). Educación Transmedia. De los contenidos generados por los usuarios a los contenidos generados por los estudiantes. Revista Latina de Comunicación Social, 74, 116 -132. http://dx.doi.org/10.4185/RLCS-2019-132

Shavelson, R. J., Zlatkin-Troitschanskaia, O., Beck, K., Schmidt, S., \& Marino, J. P. (2019). Assessment ofuniversity students'critical thinking: Next generation performance assessment. International Journal of Testing, 19(4), 337-362.

https://doi.org/10.1080/15305058.2018.1543309
Symonds, J., Schoon, I., Eccles, J., \& Salmela-Aro, K. (2019). The development of motivation and amotivation to study and work across age-graded transitions in adolescence and young adulthood. Journal of Youth and Adolescence, 48, 1131-1145. https://doi. org/10.1007/s10964-019-01003-4

Tang, Y., \& Hew, K. F. (2017). Using Twitter for education: Beneficial or simply a waste of time? Computers \& education, 106, 97-118.

https://doi.org/10.1016/j.compedu.2016.12.004

Welbers, K., Konijn, E. A., Burgers, C., de Vaate, A. B., Eden, A., \& Brugman, B. C. (2019). Gamification as a tool for engaging student learning: A field experiment with a gamified app. E-Learning and Digital Media, 16(2), 92-109.

https://doi.org/10.1177/2042753018818342

Zhao, Y., \& Hoge, J. D. (2005). What elementary students and teachers say about social studies. The social studies, 96(5), 216-221.

https://doi.org/10.3200/TSSS.96.5.216-221

Zichermann, G., \& Cunningham, C. (2011). Gamification by design: Implementing game mechanics in web and mobile apps. O'Reilly Media, Inc. 


\section{ALTERIDAD}

p-ISSN: 1390-325x / e-ISSN: 1390-8642

Vol. 17, No. 1/ enero-junio 2022

\section{Sección Miscelánea}

(Miscellaneous Section)

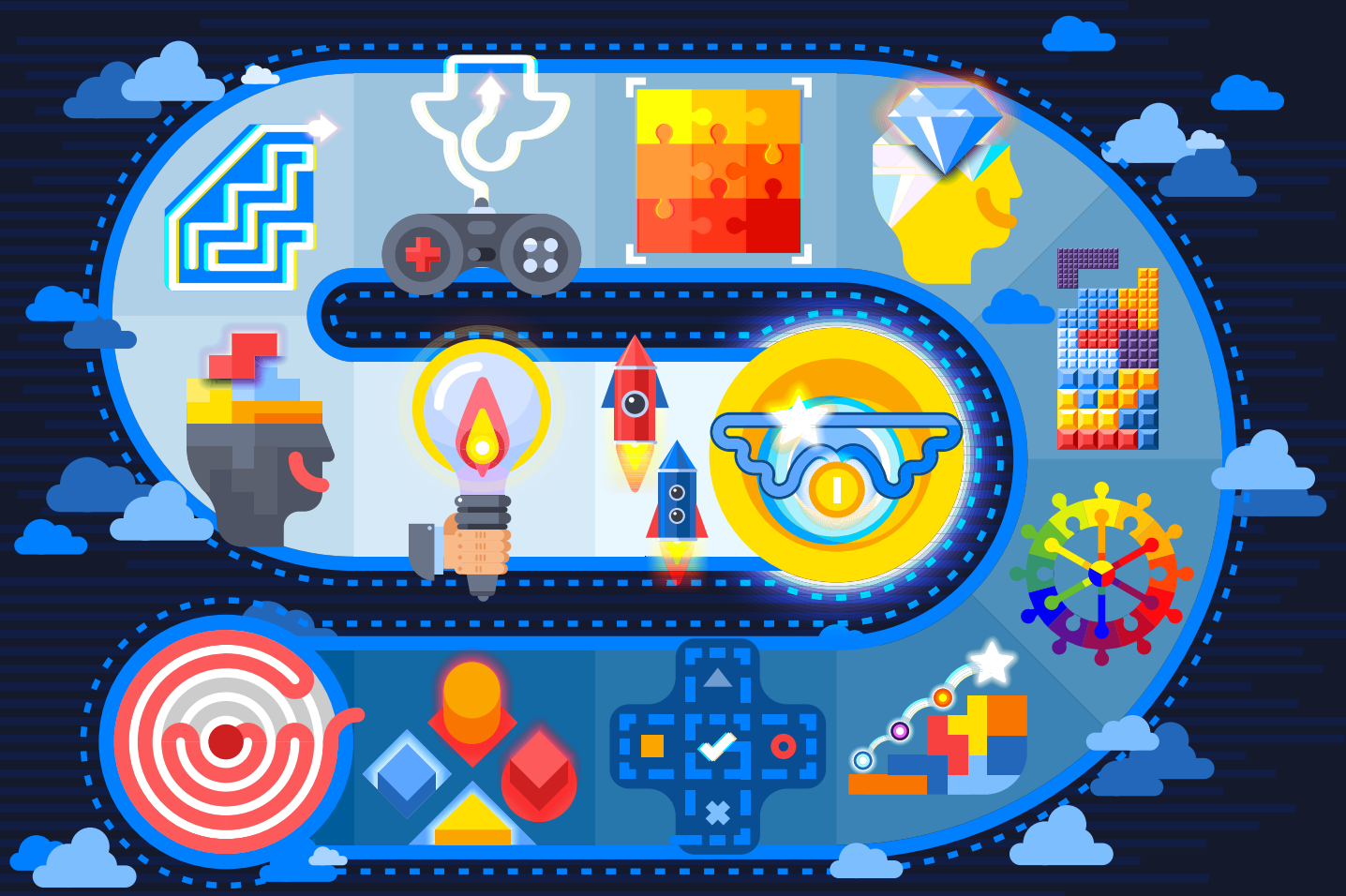

Fuente: https:/ / www.shutterstock.com/es/image-vector/gamification-techniques-business-education-behavior-change-795756976 


\section{EATRRDAD Aprendizaje servicio en educación superior entre España y México. Hacia los ODS}

\section{Service-learning in higher education between Spain and Mexico. Towards the SDGS}

D Dr. Fernando González-Alonso es profesor e investigador Universidad Pontificia de Salamanca (España) (fgonzalezal@upsa.es) (https://orcid.org/0000-0002-6507-3433)

D Dra. Azucena Ochoa-Cervantes es profesora e investigadora de la Universidad Autónoma de Querétaro (México) (azus@uaq.mx) (https://orcid.org/0000-0003-4515-9069)

D Dr. José Luis Guzón-Nestar es profesor del CES Don Bosco-Universidad Complutense de Madrid (España) y del San Pío X Universidad Pontificia de Salamanca (España) (jguzon70@gmail.com) (https://orcid.org/0000-0002-1526-5058)

Recibido: 2021-05-22 / Revisado: 2021-09-12 / Aceptado: 2021-11-26 / Publicado: 2022-01-01

\section{Resumen}

El artículo considera el Aprendizaje Servicio (ApS) universitario entre España y México, ante la inexistencia de revisiones literarias sobre el ApS y con la finalidad de generar motivación y compromiso, a través de las experiencias propuestas y analizadas. Para lo cual, aborda las raíces, los fundamentos filosóficos y pedagógicos y los modelos que lo articulan y justifican como elementos de sumo interés y actualidad. Identifica experiencias de ApS, diseñadas desde universidades de España y México, que respondan a las dimensiones que se proponen y tiendan a los Objetivos para el Desarrollo Sostenible (ODS), para conocerlas, analizarlas, compararlas y valorarlas. Las experiencias son aplicadas por estudiantes universitarios, con el apoyo de organismos en red, como servicio a la comunidad, en sectores sociales vulnerables de infancia, adolescencia y juventud, de comunidades indígenas, mujer, etc. El alumnado experimenta un notorio aprendizaje, al sumar teoría y práctica en situaciones reales, relacionadas con arte y patrimonio cultural, educación física, formación de maestros, educación ambiental y ciudadana, participación solidaria, igualdad de género y el desarrollo y acompañamiento psicopedagógico. Muestra un marco referencial y prospectivo, convirtiendo el ApS de las dos orillas, en estrategias cooperativas de enseñanza, servicios y aprendizajes socioeducativos, que acercan universidad y sociedad, para la mejora académica, el crecimiento personal y grupal y la participación cívica y democrática, favoreciendo el compromiso hacia la justicia social y el logro progresivo de los ODS.

Descriptores: Aprendizaje-Servicio, educación superior, servicio social, España, México, Objetivos Desarrollo Sostenible.

\section{Abstract}

The article considers university Service Learning (SL) between Spain and Mexico, in the absence of literary reviews on SL and with the aim of generating motivation and commitment, through the experiences proposed and analyzed. For which, it addresses the roots, the philosophical and pedagogical foundations and the models that articulate and justify it as elements of great interest and relevance. Identify PHC experiences, designed by Universities in Spain and Mexico, that respond to the dimensions that are proposed and tend to the Sustainable Development Goals (SDG), in order to know, analyze, compare and value them. The experiences are applied by university students, with the support of network organizations, as a service to the community, in vulnerable social sectors of childhood, adolescence and youth, indigenous communities, women, etc. The students experience a remarkable learning, by adding theory and practice in real situations, related to art and cultural heritage, physical education, teacher training, environmental and civic education, solidarity participation, gender equality and psychopedagogical development and support. It shows a referential and prospective framework, turning the SL of both shores into cooperative strategies of teaching, services and socio-educational learning, which bring university and society closer together, for academic improvement, personal and group growth and civic and democratic participation, favoring the commitment to social justice and the progressive achievement of the SDGs.

Keywords: Service-Learning, higher education, social service, Spain, Mexico, Sustainable Development Goals.

Forma sugerida de citar: González-Alonso, F., Ochoa-Cervantes A., \& Guzón Nestar, J. L. (2022). Aprendizaje servicio en educación superior entre España y México. Hacia los ODS. Alteridad, (17)1, 76-88. https://doi.org/10.17163/alt. v17n1.2022.06 


\section{Introducción}

Hace unas décadas, el Aprendizaje Servicio (ApS) como técnica innovadora estaba en la periferia de la academia. Hoy, en cambio, el ApS se ha extendido prácticamente por toda la educación superior. Pocas experiencias de innovación educativa han logrado un éxito tan rápido y global (Deeley, 2016).

En este el artículo se describen inicialmente los fundamentos históricos y filosófico-pedagógicos del ApS, así mismo se hace referencia a experiencias significativas que nos hacen comprender mejor su historia y futuro. Posteriormente, se realiza una revisión literaria sobre el ApS desarrollado desde la educación superior como servicio comunitario hacia personas y grupos más vulnerables. Las experiencias de ApS han sido desarrolladas en universidades de España y México, evidenciando sus proyectos solidarios enmarcados en el contexto de los Objetivos para el Desarrollo Sostenible (ODS) dentro de la Agenda 2030.

A partir del artículo de Robert Sigmon Service-learning: Three Principles (1979) que dio origen a esta metodología, y ayudó a establecerla y formalizarla, se han ofrecido numerosas definiciones de ApS. Algunas de las más citadas son:

ApS es una experiencia educativa vinculada a créditos universitarios en la que los alumnos participan en una actividad organizada de voluntariado que satisface las necesidades comunitarias identificadas y en la que se reflexiona en torno a la actividad, de modo que se adquiera una ulterior comprensión del contenido del curso, una apreciación más global de la disciplina y un mejor sentido de la responsabilidad cívica. (Bringle \& Hatcher, 1996, p. 221)

ApS es un método con el que los estudiantes aprenden y se desarrollan a través de la participación activa en un servicio cuidadosamente organizado que se lleva a cabo en una comunidad y satisface sus necesidades y se coordina con una institución de educación superior y con la comunidad; ayuda a fomentar la responsabilidad cívica; se integra en el plan de estudios académico de los estudiantes y lo mejora e incluye un tiempo estructurado para que los estudiantes... reflexionen sobre la experiencia de servicio. (Corporation for National and Community Service, 1993)

Finalmente, Ehrlich (1996) sostiene que:

ApS es la variedad de pedagogías que unen el servicio comunitario y el estudio académico para que cada uno fortalezca al otro. La teoría básica del ApS es la de Dewey: la interacción del conocimiento y las habilidades con la experiencia es clave para el aprendizaje. El aprendizaje comienza con un problema y continúa con la aplicación de ideas cada vez más complejas y habilidades cada vez más sofisticadas a problemas cada vez más complicados. (p. 3)

Las diversas definiciones convergen en un consenso sobre los elementos esenciales de la pedagogía ApS:

- Integración de los objetivos del aprendizaje y los objetivos del servicio.

- Cumplimentación de los objetivos de aprendizaje académico con objetivos de aprendizaje cívico (educación para la ciudadanía) y también con otras categorías de aprendizaje (crecimiento personal, desarrollo profesional, competencia intercultural, investigación ética, habilidades de investigación).

- Proceso organizado y estructurado.

- Colaboración recíproca entre estudiantes, profesores, miembros de la comunidad e institución que cumple los objetivos colectivos y crea capacidad entre todos.

- Reflexión crítica.

- Duración e intensidad suficientes para producir resultados significativos en ApS.

Desde una perspectiva histórica, el ApS es un proceso relativamente nuevo en la educación superior. El término fue acuñado por primera 
vez en 1967, en referencia a un programa de prácticas que fue patrocinado por la Southern Regional Education Board (EEUU), y a través de la cual, los universitarios obtuvieron créditos académicos y/o una remuneración financiada por el gobierno federal para trabajar en proyectos comunitarios (Sigmon, 1979).

Como práctica pedagógica en educación superior, el ApS se limitó a un pequeño grupo de participantes hasta mediados de 1980. A finales, el ApS fue ganando adeptos y se distinguía claramente de los servicios comunitarios, por su atención a la integración del servicio con el estudio académico (Hollander et al., 2001).

Los años 90 han sido testigo de un gran crecimiento del ApS, de manera que ahora se considera como una "fuerza vital en el cambio educativo" (Liu, 1996, p. XI). Los colegios y universidades han adoptado un compromiso renovado con la responsabilidad cívica y con el ApS como vehículo central para cumplir el compromiso (Ehrlich, 2000).

\section{Fundamentos filosóficos y pedagógicos del ApS}

El ApS es una estrategia de enseñanza-aprendizaje colaborativa y democrática diseñada para promover la mejora académica, el crecimiento personal y el aprendizaje cívico y para fomentar el compromiso social. En ella, el alumnado se asocia recíprocamente con los miembros de la comunidad, con los y las profesores y el personal de administración y servicios, en experiencias relacionadas con el material académico y con cuestiones de interés público. Mediante una reflexión guiada, el alumnado examina sus experiencias de manera crítica y articulan resultados de aprendizaje específicos y aplicables, mejorando así la calidad de su aprendizaje y de su servicio. Los y las estudiantes, el profesorado y los miembros de la comunidad sirven como co-educadores, co-aprendices, co-servidores y co-generadores de conocimiento (Bringle, et al., 2011).
El desarrollo de las iniciativas de ApS ha ido acompañada de un renovado interés por la filosofía de Dewey, cuyos escritos sobre la naturaleza activa y los beneficios y condiciones de la democracia participativa "proporcionan un temprano fundamento teórico para una pedagogía en la que los estudiantes se comprometan cooperativamente a resolver problemas" (Speck \& Hoppe, 2004, p. 19). Dewey (1859-1952), tiene algunas obras que quieren objetivar la importancia de la experiencia para el ser humano y especialmente en el ámbito de la educación: Experience and Objective Idealism (1907) y Experience and Nature (1925). Dewey señala que:

Aprender de la experiencia, implica establecer un vínculo activo entre el comportamiento y sus consecuencias. Es una actividad que está en oposición directa con el modelo tradicional de educación, en el que los alumnos son imbuidos de pasividad. Se aboga por un aprendizaje activo a través de la experiencia, partiendo de la creencia de que 'la mayoría del aprendizaje no es resultado de la instrucción. Es más bien el resultado de la libre participación en un escenario significativo' (Illich, 1971, p. 39). [...] Dewey critica este tipo de educación y aboga por una pedagogía más progresista, en la que el alumno tiene oportunidades para implicarse activamente en su propio aprendizaje. La participación activa concierne a la experiencia. No es la experiencia en sí misma porque debe ser educativa, y solo es educativa cuando se adquiere conocimiento significativo. (Deeley, 2016, p. 51)

Sin embargo, no cabe pensar que la filosofía de Dewey es el único fundamento (Giles \& Eiler, 1994), hay otras rutas que explorar. Por consiguiente, conviene afirmar que el ApS es una filosofía de "crecimiento y finalidad humana, una visión social, un enfoque de comunidad y una forma de saber" (Kendall, 1990, p. 23). Es una pedagogía:

Basada en la experiencia como base para el aprendizaje y en la centralidad e intenciona- 
lidad de la reflexión diseñada para permitir que el aprendizaje suceda. Se basa en el trabajo de investigadores y teóricos del aprendizaje, incluyendo a Dewey, Piaget, Lewin, Schon y Kolb, que creen que aprendemos a través de combinaciones de acción y reflexión. (Jacoby, 2003, p. 4)

Aquí convendría fijarse en la aportación de Dewey, que especialmente "critica la reproducción acrítica de conocimiento y cree que los alumnos deben aprender a resolver problemas de modo competente o desarrollar una 'actitud de la mente que conduzca al buen juicio"' (Deeley, 2016, p. 51).

Da mucha importancia a la creación de sentido, a la relación que se establece entre pensamiento y lenguaje. Por consiguiente, también es importante contar con la reflexión de Vygotsky (1962) por lo que en sus fundamentos está muy vivo el constructivismo social o el socio constructivismo. Deeley (2016) nos ayuda:

El constructivismo social, o la teoría del constructo social, es un valioso componente dentro del marco teórico del ApS. Aquí también se incluye la teoría del constructo personal, que concierne a cómo la gente comprende o dota de sentido sus experiencias personales. [...] A través de la reflexión crítica sobre sus experiencias y trabajo académico, resulta evidente que los alumnos modifican y deconstruyen sus ideas de partida sobre sí mismos y su comprensión del mundo. (p. 41)

Por otra parte, en el ApS se retoma el aprendizaje colaborativo, que resulta "relevante para un paradigma teórico del ApS porque los alumnos están necesariamente implicados en un proceso de puesta en común del pensamiento reflexivo dentro de un entorno de tutoría en grupo reducido" (Deeley, 2016, p. 44).

Aunque el ApS está dirigido principalmente al mundo universitario, en la construcción de su paradigma incluimos el ámbito del aprendizaje de adultos. Siguiendo la estela de Knowles y Jarvis, esta técnica innovadora tiene componentes de este tipo de aprendizaje, por el hecho de que la experiencia de vida puede conducir a los adultos a cuestionar por qué y cómo aprenden (Deeley, 2016, p. 54).

Otra influencia que ha fecundado el ApS es la teoría del aprendizaje transformativo o transformador. La transformación no suele ser patrimonio de la enseñanza en los primeros niveles educativos, sino entre adultos. Cuando hablamos de asociaciones y transformaciones entre campus y comunidades, nos referimos a cambios significativos en la forma en que las universidades entienden el mundo. "La transformación promete una mayor comprensión holística y coherente de nuestras situaciones comunes" (Jacoby, 2003, p. 39). Así, los factores clave del aprendizaje transformativo comprenden la experiencia, la reflexión crítica y el desarrollo (Merriam et al., 2007).

Hay otras visiones que se han proyectado sobre el ApS. Por ejemplo, quienes piensan que se fundamenta en la teoría crítica y la pedagogía feminista (Brown 2001; Deans, 1999). Como señalan Freire (1967) y Shor (1987), la teoría crítica hace hincapié en que la educación es política y debe incluir un enfoque dialéctico de la presentación de problemas y una crítica de los sistemas sociales y las responsabilidades civiles de la educación. La pedagogía feminista también propugna la necesidad de reflejos críticos y un diálogo relacionado con los aspectos educativos del privilegio y el poder (Weiler, 1991). Estos modelos apuntan a la importancia de situar el ApS en el contexto de las cuestiones y desafíos sociales. El desarrollo de la participación y la investigación basada en la comunidad en materia de ApS, son ejemplos de la forma en que estas pedagogías apoyan la promoción comunitaria, y dan mayor voz a la comunidad con sus fortalezas y necesidades (Strand et al., 2003; Reardon, 1998). Además del uso de modelos de aprendizaje social y aprendizaje cognitivo, entre los avances recientes en el marco teórico del ApS y la construcción de su paradigma, se encuentran el uso de una pedagogía del compromiso (Lowery 
et al., 2006) y el ApS como pedagogía posmoderna (Butin, 2005).

$\mathrm{Al}$ igual que Jacoby (2003), se puede esperar que el ApS no se convierta en:

Algo educativo que los investigadores documentan en una revisión histórica publicada en 2030 como una moda pasajera, una innovadora pedagogía, que desapareció a medida que las influencias corporativas reconstruyeron la universidad moderna en un vehículo eficiente para impartir educación estandarizada a bajo costo, pero altamente rentable, la moda. (p. 39).

Las filosofías e iniciativas del ApS integran los modelos anteriores y van añadiendo históricamente otros paradigmas emergentes que surgen en la práctica. Es un buen ejemplo del espíritu que debiera reinar en nuestras comunidades educativas siempre en búsqueda de prácticas de promoción y transformación.

El ApS, sus antecedentes y fundamentos filosófico-pedagógicos, nos sitúan ante un panorama de experiencias del ApS que han evolucionado, según explican Speck y Hoppe (2004) hacia modelos básicos: el filantrópico, basado en la caridad y la filantropía, frente a problemas sociales, mitigados por el altruismo y la justicia; el modelo de participación cívica, donde profesores y alumnos son los agentes que enseñan y sirven cívica y democráticamente a la sociedad; y el comunitario, proyectado en la comunidad local, salvaguardando virtudes y derechos. Posteriormente, García-Romero y Lalueza (2019) consideran tres marcos teóricos sobre los que clasifican el ApS universitario: 1) El aprendizaje experiencial, centrado en el aprendiz que aprende y encuentra sentido y significado en lo que hace para la comunidad; 2) el aprendizaje transformativo, que ha de producir disonancia y nuevo vocabulario por el cambio radical que se produce; y 3) las teorías críticas, con una pedagogía con proyección de transformación comunitaria.

\section{Las experiencias de ApS: metodología, dimensiones y Objetivos para el Desarrollo Sostenible}

Este trabajo es producto de una investigación documental. Como parte de la metodología, las experiencias de ApS que presentamos, se basan en los modelos expuestos, o integran uno mixto, abarcando la acción altruista, participativas en el entorno educativo, con proyección socio comunitaria y de aprendizaje experiencial $y$ transformativo.

Identificamos ocho experiencias de ApS universitarias, cuatro de España y cuatro de México, países de nuestro origen cultural e interés profesional con proyección de ApS, para conocerlas, analizarlas y valorarlas comparativamente a través de dimensiones e indicadores concretos, dado que el grueso importante de publicaciones de ApS se centran en el marco teórico (Chiva et al., 2016; Zayas et al., 2018; Rodríguez-Izquierdo, 2020). Entre las experiencias de ApS publicadas, seleccionamos las planteadas como proyecto articulado desde la universidad, con reconocimiento $\mathrm{y}$ apoyo institucional, que destaquen por sus elementos identificativos, problemas de salud, educación, cultura, medio ambiente, etc., con relación especial a los Objetivos de Desarrollo Sostenible (ODS) (ONU, PNUD, 2015).

Vinculamos las experiencias de ApS con los ODS de la Agenda 2030 porque comparten finalidades que garantizan la paz y el progreso de las sociedades más vulnerables, ya que los 17 ODS buscan el fin de la pobreza (1) y del hambre (2); garantizan la vida sana (3), la educación inclusiva, equitativa, de calidad y el aprendizaje a lo largo de la vida (4); avalan la disponibilidad y sostenibilidad del agua, el saneamiento (6) y la energía asequible y segura (7); promueven el crecimiento económico sostenido y sostenible, el empleo pleno, productivo y decente (8); suscitan sociedades pacificas e inclusivas, el acceso equitativo a la justicia y la 
creación de instituciones eficaces y responsables (16); promueven ecosistemas terrestres y bosques sostenibles, luchan contra la desertificación, la degradación de las tierras y la pérdida de la diversidad biológica (15). Pretenden la igualdad de géneros y el empoderamiento de mujeres y niñas (5); procuran ciudades y asentamientos humanos inclusivos, seguros y sostenibles (11); la reducción de la desigualdad entre países (10); garantizan el consumo y producción sostenible
(12); promueven infraestructuras e industrias inclusivas y sostenibles (9); combaten el cambio climático y sus efectos (13), la sostenibilidad de océanos, mares y recursos marinos (14); buscan reducir la desigualdad entre países y revitalizar la alianza para el desarrollo (17).

En las experiencias de ApS, como criterios de análisis y valoración, tomamos dimensiones e indicadores (Bellera et al., 2014; Campo, 2015; Escofet et al., 2016) de la tabla 1.

Tabla 1. Dimensiones para la valoración de experiencias universitarias de Aps

\begin{tabular}{|l|l|l|l|}
\hline 1. Planificación pedagógica & 2. Aprendizaje & 3. Servicio & 4. Participación \\
\hline 5. Competencias & 6. Axiología y valores & 7. Trabajo en red y la universidad & 8. Vocacional y profesional \\
\hline $\begin{array}{l}\text { 9. Coordinación, evaluación y } \\
\text { satisfacción }\end{array}$ & & \\
\hline
\end{tabular}

Elaboración propia a partir de Bellera et al., 2014; Campo, 2015; Escofet et al., 2016.

\section{Experiencias de ApS universitarias en España}

Algunas experiencias de ApS diseñadas en grados universitarios de España, pretenden solucionar demandas y necesidades sociales, evidenciando elementos identificativos y analizando las experiencias desde las dimensiones señaladas. La aplicación de proyectos de ApuniS permite a los participantes vivir experiencias únicas $\mathrm{y}$ significativas reales, con proyección formativa y vocacional, logro de la ciudadanía activa y a favor de la comunidad (Pérez-Galván \& Ochoa, 2017).

\subsection{ApS: una biblioteca para escuela infantil}

Alumnos del último año de Educación Infantil de la Universitat Autónoma de Barcelona, aplicaron un proyecto de ApS (González et al., 2015) creando una biblioteca infantil para una escuela con alumnado de dos a seis años, durante las prácticas externas. Aprenden como maestros futuros analizando las necesidades, sistemati- zando información, presentando el proyecto y presupuesto al equipo directivo de la escuela, decidiendo la metodología y los recursos y realizando un servicio al centro. La red de trabajo y colaboración se da entre la comunidad universitaria, tutores de prácticas, autoridades escolares, padres y miembros de bibliotecas.

La coordinación entre los organismos se da a través de las reuniones grupales y la planificación de actividades, como visitas a la biblioteca, presentación de normas y su funcionamiento, elaboración de pictogramas y fotos, organización de libros, realización de dibujos... Los niños y niñas son los beneficiarios. Todos se motivan por la interrelación y la participación en el proyecto. Adquieren competencias al buscar y gestionar información y trabajar en equipo. Influye directamente sobre créditos de la asignatura del Practicum, mostrando valores educativos y sociales. El proyecto tiene un carácter vocacional y profesional hacia las competencias del maestro, percibidas en la evaluación final. Se logran ODS como la educación inclusiva, la promoción de oportunidades de aprendizaje y de sociedades 
pacíficas e inclusivas, la igualdad de género y el empoderamiento femenino.

\subsection{ApS: el arte de aprender Arte. ApS para construir identidad profesional}

Gregori-Giralt y Menéndez-Varela (2014) publican la experiencia de ApS basada en el diseño de proyectos de arte por parte del alumnado de asignaturas de formación básica de las titulaciones de Bellas Artes de la Universidad de Barcelona. Como artistas, diseñadores y conservadores de arte, orientaron sus proyectos a resolver alguna de las dificultades de la comunidad, analizando sus necesidades, y aplicando los conocimientos de la materia. Las calificaciones sobre los proyectos fueron mejorando en función de la identidad profesional de los grupos con el compromiso social. Lograron las dimensiones de planificación pedagógica, aprendizaje y servicio en la visión y misión del mundo que les rodea; la vinculación institucional y la conciencia e identidad vocacional. Alcanzaron ODS como la educación inclusiva, equitativa, de calidad y el aprendizaje y la reducción de las desigualdades, etc. La experiencia muestra la planificación y el aprendizaje cooperativo en torno al arte, su diseño y conservación. Se destaca el alto valor social y artístico, la implicación y el apoyo de la universidad, transcendiendo la conciencia e identidad profesional que conecta el arte con grupos humanos más excluidos.

\subsection{ApS: un maestro para Datem}

"Un maestro para el Datem" es una experiencia de ApS, en la que participan como prácticas externas, alumnado de Educación Infantil y Primaria de la Facultad de Educación de la Universidad Pontificia de Salamanca (UPSA). Siguiendo un modelo universitario de educación en valores (Alonso \& De Castro, 2015; Martín-García, et al., 2021), colaboran en red en el Proyecto de la Universidad Marcelino Champagnat de Lima
(UMCH), junto a la ONGD SED y el Instituto de Estudios Maristas (IEM), entre otros. El servicio se hace al alumnado de comunidades indígenas de siete etnias: Achuar, Awajún, Chapra, Shawi, Quechua, Wampis y Kanz, junto a mestizos, que acuden durante siete años en períodos vacacionales a San Lorenzo, provincia de Datem del Marañón en la Amazonía peruana, durante dos veces al año, una para entregar trabajos académicos (diciembre y agosto) y otra para recibir clases (enero).

Al proyecto acuden más de 500 indígenas adultos de Educación Inicial y Educación Primaria (Pérez, 2015), para obtener la titulación que les permita capacitarse y mantener la plaza en escuelas de sus comunidades repartidas por la selva de Perú. En enero, el alumnado de UPSA, junto a otros compañeros, desarrollan las prácticas docentes de asignaturas que han preparado fervientemente. Desarrollan tareas domésticas que elogian la convivencia. Por parte de $\mathrm{UMCH}$, el alumnado de UPSA recibe información, materiales, programación de actividades, apoyo y formación necesaria para el desarrollo de sus funciones en el respeto a fines, normas y desde la confidencialidad, sin contraprestación alguna, procurando el bien superior de los destinatarios.

El aprendizaje del alumnado agente se basa en el protagonismo ejercido, las competencias adquiridas, los valores socioeducativos y la calidad al servicio planificado. Se observa conciencia vocacional y profesional en la actividad docente, con valoraciones e índices altos de satisfacción. La experiencia acerca los ODS: disminuyen la pobreza, garantizan la educación inclusiva, equitativa y de calidad, reducen la desigualdad, logran asentamientos humanos inclusivos, seguros y sostenibles y fortalecen los medios de ejecución, revitalizando la alianza mundial para el desarrollo sostenible. 


\subsection{ApS: educación física para jóve- nes con discapacidad intelectual}

La experiencia de ApS (Santos-Pastor et al., 2018) desarrolló un proyecto de educación física y deporte para jóvenes con discapacidad intelectual del Programa Promentor (formación para la inclusión laboral de jóvenes con discapacidad intelectual). Alumnado del grado de CAFyD y de Educación Primaria de la mención de educación física de la Universidad Autónoma de Madrid, con sus respectivos docentes, formaron una red de trabajo con sesiones elementales planificadas de educación física, desarrollando roles que favorecieron el aprendizaje de unos y el servicio de otros. La participación, la motivación, los valores y las competencias logradas, se confirman en la evaluación basada en un cuestionario y un diario, que revelan la satisfacción y aportan mejoras. Se alcanzaron los ODS que garantizan una vida más sana y de bienestar; una educación inclusiva, equitativa, de calidad y de oportunidades y asentamientos humanos más inclusivos, resilientes y sostenibles.

\section{Experiencias de ApS universitarias en México}

- En México, uno de los primeros antecedentes que reconocemos es el servicio social obligatorio que todas y todos los estudiantes de Educación Superior cumplen para acceder a un título de grado en cualquier carrera. Surge en el contexto de la Revolución Mexicana (1910) y se implementa como una forma de compensar las carencias y desigualdades que se dan, producto de este movimiento. Según Mungaray et al. (2002), el servicio social en la Educación Superior mexicana se constituyó como una actividad espontánea, de alta solidaridad y reciprocidad por parte de estudiantes universitarios. En 1945 se reforma el artículo 50 constitucional, donde se establece "el servicio social obligatorio para estudiantes de educación media superior y superior. Convirtiéndolo en una institución básica del desarrollo social en México" (p. 33).

Actualmente el servicio social permanece como requisito obligatorio para obtener el título profesional, acorde con lo dispuesto en la Ley General de Educación, así, los futuros profesionistas deben cumplir con 480 horas de servicio. Sin embargo, si bien se puede identificar como antecedente, no todos los programas de servicio social universitario se caracterizan como proyectos de ApS, debido, entre otras cosas, a que no siempre están explícitamente vinculados a aprendizajes y que cada institución determina los mecanismos para desarrollarlo.

Para el objetivo de este documento se optó por seleccionar dos prácticas de servicio social que, aunque no tuvieran la "etiqueta" de ApS contaran con elementos para considerarlos como tal, se describen otras dos donde las autoras retoman el ApS explícitamente. Para su análisis y valoración se siguió las dimensiones e indicadores descritos.

\subsection{ApS en intervenciones de educación ambiental}

El documento "Experiencias de intervención sobre educación ambiental se sustentan en comunidades de Guerrero" (Figueroa et al., 2016), desarrolla el servicio social de tres generaciones de estudiantes en la licenciatura Desarrollo Regional de la Universidad de Guerrero (UAGRO). Describe el análisis de la contribución de los proyectos de servicio social al campo de la Educación Ambiental sustentable.

Las intervenciones se centran en talleres de educación ambiental: capacitación para la producción de hortalizas, para el manejo de residuos sólidos y para la construcción de estufas ecológicas dirigida a mujeres. Estas intervenciones contribuyen a los ODS al garantizar una vida sana y promover el bienestar para todos; garantizar una educación inclusiva, equitativa y 
de calidad y promover oportunidades de aprendizaje; garantizar el acceso a una energía asequible, segura, sostenible y moderna y garantizar el consumo y producción sostenibles.

En cuanto a la planificación pedagógica, se observan aprendizajes centrados en contenidos específicos sobre educación ambiental y salud. Relacionan la sustentabilidad con la salud humana y animal. El servicio se ve claramente definido en la construcción de estufas, donde el alumnado realizó acuerdos con la comunidad para diagnosticar la salud, afectada por problemas respiratorios asociados a la inhalación de humo de los fogones y la puesta en marcha, no solo de la construcción de las estufas, sino de un programa de capacitación en medioambiente y la salud. La experiencia recoge el protagonismo del alumnado, al dar respuesta a partir de los conocimientos que contaban, al realizar todas las actividades y su logística, contribuyendo su visión del aprendizaje y del mundo, al tratarse de comunidades muy vulnerables de regiones pobres y con grandes problemáticas educativas y sociales de México. Se observa la importancia de la vinculación entre universidad y comunidad por la relación horizontal, para lograr aprendizajes a través del servicio que satisfizo necesidades reales. Hay que destacar que, al tratarse de prácticas de servicio social, no observamos procesos de reflexión y evaluación, como parte sustancial de los proyectos ApS.

\subsection{ApS sobre participación solida- ria y equidad de género}

Un artículo de Quiroga (2013) de la Universidad de Monterrey (UDEM), da cuenta del impacto de la metodología ApS en el empoderamiento de la mujer. La intervención realizada por estudiantes del curso de pregrado de Aplicaciones en Psicología Clínica, desarrolló un programa para impulsar la capacidad en la toma de decisiones de las mujeres. Los objetivos de aprendizaje se centraron en la identificación y diagnóstico de problemas del comportamiento, cognitivos, emocionales y sociales. Se midieron datos cuantitativos sobre el índice y severidad de la violencia de pareja en las mujeres adultas, que asisten a los espacios de servicio a la comunidad y variables psicológicas, que moderan y median su presencia. Las actividades se realizaron en colaboración con el DIF (Desarrollo Integral de la Familia) un organismo del gobierno federal y estatal.

Esta experiencia contribuye a "lograr la igualdad entre los géneros y empoderar a todas las mujeres y las niñas" como ODS, aunque no esté explicitado. Debido a que el artículo se centra en mostrar el impacto de la intervención realizada, no podemos dar cuenta del proceso del proyecto. Se incluyeron estrategias que garantizaban el servicio de calidad, como el diagnóstico participativo con las instituciones beneficiarias; el asesoramiento por parte del docente; los vínculos con organizaciones comunitarias y las actividades planeadas y gestionadas.

\subsection{ApS en la implementación de política pública para la infancia}

Desde la Universidad de Monterrey, LópezVillarreal (2018) publicó el artículo "Aprendizaje Servicio en la implementación de política pública para la infancia", describiendo una intervención realizada con metodología vinculada a la asignatura de Teorías del Aprendizaje, al fortalecer:

El área psicopedagógica de ocho Casas Hogar, mediante el desarrollo de manuales pedagógicos enfocados a las necesidades educativas de los niños que habitan estas instituciones (...). La experiencia analiza las relaciones entre los estudiantes que favorecieron la educación ciudadana implementando el ApS como proceso de las políticas públicas. (p. 1)

Se infiere que no se describe ni el proceso, ni los resultados. Los ODS cercanos al logro son la educación inclusiva, equitativa y de calidad y las sociedades pacíficas e inclusivas para el desarrollo sostenible y el acceso a la justicia. 


\subsection{ApS en torno al Programa "Adopta a un amig@"}

Son 55 Instituciones de Educación Superior (IES) que participan en el programa internacional de servicio social PERAJ "Adopta a un amig@”. El objetivo es apoyar el desarrollo psicosocial de estudiantes de primaria, mediante el acompañamiento de jóvenes universitarios a través de la tutoría, haciendo orientación académica, mejora de la autoestima, habilidades sociales y aumento de su horizonte de vida, para vencer el rezago social y educativo. El programa contribuye a los ODS: Garantizar una vida sana y promover el bienestar para todos en todas las edades; garantizar una educación inclusiva, equitativa y de calidad y promover oportunidades de aprendizaje durante toda la vida, aunque no se expliciten. El servicio de los universitarios está bien definido, al enfocarse en la disminución de riesgos psicosociales, el fortalecimiento de la cohesión social y el fomento de formación integral y compromiso social.

En la revisión localizamos dos artículos que muestran el impacto de este programa en la formación del universitario. El primero denominado Evaluación del programa de servicio social UADY- Peraj "Adopta a un amig@” (Santoyo, 2017), si bien no describe la forma de llevarlo a cabo, muestra el impacto positivo en el alumnado de primaria y la discusión del autor por redefinir los tiempos y duración de desarrollo del programa. En el caso del estudio "análisis del impacto del programa social UBP Peraj "Adopta un amig@” con estudiantes de la Universidad Politécnica del Bicentenario (Gómez \& Escobedo, 2019), se centra en la evaluación del alumnado, destacando su honestidad, escucha activa, paciencia y compromiso social.

\section{Conclusiones}

Atendiendo a la naturaleza del ApS (origen y finalidad), avalamos que el ApS es una estrategia de enseñanza-aprendizaje colaborativa y democráti- ca, diseñada para promover la mejora académica, el crecimiento personal y el aprendizaje cívico. Fomenta las necesidades públicas, en la que el alumnado se asocia recíprocamente con miembros de la comunidad y de la universidad en experiencias, tanto académicas como de interés público. En el fondo, uno de los objetivos más importantes de ApS es acercar la universidad a la sociedad.

Experiencias de ApS universitarias como estas enriquecen a toda la comunidad universitaria y a quienes participan de una u otra manera, produciendo una verdadera red de trabajo cooperativo, que revela el sentido vocacional y profesionalizante hacia la comunidad y el aprendizaje mutuo.

En el ámbito universitario español, a diferencia del méxicano, este tipo de experiencia está a veces vinculada a la práctica de las materias, y en otras es voluntaria. La experiencia de ApS no siempre va asociada al reconocimiento de créditos formativos.

En la revisión de ApS en México, existe carencia en la sistematización de experiencias, tanto de servicio social como de ApS en las IES. Si partimos de que la sistematización permite ordenar y clasificar las experiencias, para comprobar su planificación, definición y dimensiones de actuación, para aprender de los aciertos y errores, y así construir aprendizajes y mejorar las prácticas, en México nos queda un gran trecho por recorrer. Para poder dar cuenta de la mejora a partir de la introducción de esta metodología de ApS que, si bien tiene una larga tradición, al parecer no ha permeado en las propuestas de innovación pedagógica de las IES. Más aún, es necesario evaluar y reconsiderar el servicio social universitario, ya que la falta de sistematización, no permite valorar si se cumplen los objetivos.

Las dimensiones coincidentes en las experiencias de ApS de ambos países destacan las relacionadas con el aprendizaje, el servicio, las competencias, la participación y la evaluación. En el caso español también sobresalen la planificación, su ordenación, sistematización y el trabajo en red. 
Entre los ODS concurrentes hay que destacar los que aparecen en experiencias con marcado carácter educativo e inclusivo: garantizar la educación inclusiva, equitativa y de calidad y promover oportunidades de aprendizaje; lograr la igualdad entre los géneros y empoderar a mujeres y niñas; promover sociedades pacíficas e inclusivas para el desarrollo sostenible y facilitar el acceso a la justicia. Otros ODS como poner fin al hambre; garantizar el agua; el crecimiento económico; las infraestructuras y la industrialización; la conservación de océanos y mares, permanecen ausentes entre las finalidades de los ApS propuestos.

Los destinatarios de las experiencias de ApS de los dos países son personas de colectivos sociales excluidos como discapacitados intelectuales, menores, mujeres, indígenas, etc., que, mediante acciones de educación ambiental y ciudadana, igualdad y empoderamiento, desarrollo psicopedagógico y social, acompañamiento $y$ orientación, acercan los ODS como personas con los mismos derechos.

En general, las experiencias de ApS crean sostenibilidad educativa y curricular en la propia universidad, que ve fortalecido su sentido, propósito, inclusión social y deber solidario. De igual forma, favorecen en los participantes el conocimiento, la sensibilización, el compromiso y logro progresivo de los ODS mediante las actuaciones planeadas.

Los próximos trabajos sobre el ApS podrán centrarse en la aproximación o distanciamiento de los modelos conocidos, el seguimiento longitudinal de los agentes del ApS desde sus inicios escolares hasta su actual experiencia universitaria. Así como orientar la revisión hacia el cambio conceptual producido sobre el ApS. Desde la fundamentación filosófica y pedagógica, interesa revisar los elementos aplicados, así como continuar en el logro de los ODS desde las experiencias del ApS.

\section{Referencias bibliográficas}

Alonso, F., \& De Castro, R.M. (2015). Solidaridad y voluntariado: un modelo universitario de educación en valores. En J. Escudero y F. González (Coords.), Solidaridad y voluntariado universitario. Relatos de Experiencias (pp. 33-53). Servicio de Publicaciones (UPSA).

Bellera, J., Albertín, P., \& Bonmati, A. (2014). Criterios para valorar propuestas universitarias de Aprendizaje Servicio (ApS). Procedia - Social and Behavioral Sciences, 196 (2015), 14-20. https://doi.org/10.1016/j.sbspro.2015.07.003

Bringle, R., \& Hatcher, J.A. (1996). Implementing Service Learning in Higher Education, Journal of Higher Education, 67(2), 221-239. https://bit.ly/3EyUFrm

Bringle, R.G., Hatcher, J.A., \& Jones, S.G. (Eds.). (2011). International service learning. Conceptual frameworks and research. Sterling, VI: Stylus Publishing.

Brown, D. (2001). Pulling it together: A method for developing service-learning and community partnerships based on critical pedagogy. Corporation for National Service.

Butin, D.W. (2005). Service-Learning as Postmodern Pedagogy. En Service-Learning in higher education (pp. 89-104). Palgrave Macmillan.

Campo, L. (2015). Una rúbrica para evaluar y mejorar los proyectos de aprendizaje servicio en la universidad. RIDAS, Revista Iberoamericana de Aprendizaje Servicio, 1, 91-111.

http://dx.doi.org/10.1344/ridas2015.1.6

Chiva, Ò., Gil, J., Corbatón, R., \& Capella, C. (2016). El aprendizaje servicio como propuesta metodológica para una pedagogía crítica. RIDAS, Revista Iberoamericana de Aprendizaje Servicio, 2, 70-94.

http://dx.doi.org/10.1344/RIDAS2016.2.4

Corporation for National and Community Service (1993). The National and Community Service Trust Act of 1993. https://bit.ly/36LXKEB

Deans, T. (1999). Service-learning in two keys: Paulo Freire's critical pedagogy in relation to John Dewey's pragmatism. Michigan Journal of Community Service Learning, 6, 5-29.

https://bit.ly/32TkbWW 
Deeley, S.J. (2016). El Aprendizaje-Servicio en educación superior. Teoría, práctica y perspectiva crítica. Madrid: Narcea.

Dewey, J. (1938). Experience and education. Macmillan Publishing Co.

Ehrlich, T. (1996). Prólogo. En B. Jacoby and Associates, Service-Learning in Higher Education: Concepts and Practices. Jossey-Bass.

Ehrlich, T. (Ed.) (2000). Higher education and the development of civic responsibility. En T. Ehrlich (Ed.), Civic responsibility and higher education (pp. XXI-XIII). Americ.

Escofet, A., Folgueiras, P. Luna, E., \& Palou, B. (2016). Elaboración y validación de un cuestionario para la valoración de proyectos de aprendizaje-Servicio. Revista Mexicana de Investigación Educativa, 21(70), 929-949.

https://bit.ly/3EmeRg8

Figueroa, O., Chávez, R., \& Hilario, A, (2016). Experiencias de intervención sobre educación ambiental sustentable en comunidades de Guerrero. 1er Congreso Nacional de Educación Ambiental para la Sustentabilidad. https://bit.ly/3eGGPpT

Freire, P. (1967). Educación como práctica de la libertad. Paz e Terra.

García-Romero, D., \& Lalueza, J. L. (2019). Procesos de Aprendizaje en Aprendizaje-Servicio Universitario: Una Revisión Teórica. Educación XX1, 22(2).

http://dx.doi.org 10.5944/educxx1.22716

Giles, D., \& Eyler, J. (1994). The Impact of a College Community Service Laboratory on Students. Personal, Social and Cognitive Outcomes, Journal of Adolescence, 17(4), 327-339. https://doi.org/10.1006/jado.1994.1030

Gómez, I., \& Escobedo, P. (2019). Análisis del impacto del programa social UPB Peraj adopta a un amig@en estudiantes de la Universidad Politécnica del Bicentenario. Memoria electrónica del Congreso Internacional de Investigación. Academia Journals. México. ahttps://bit.ly/2ySMsC2

González, A., Peña, C., Prat, M., \& Rodríguez, L. (2015). Una biblioteca para la Escuela Infantil 0-6. Una propuesta de Aprendizaje Servicio. Procedia-Social and Behavioral Sciences, 196, 142-148.

http://dx.doi.org/10.1016/j.sbspro.2015.07.024
Gregori-Giralt, E., \& Menéndez-Varela J. L (2014). El arte de aprender arte: El aprendizajeservicio como instrumento para construir la identidad profesional del estudiante. Una propuesta para los estudios universitarios de arte. Procedia-Social and Behavioral Sciences, 196, 100-105.

http://dx.doi.org/10.1016/j.sbspro.2015.07.018

Hollander, E., Saltmarsh, J., \& Zlotkowski, E. (2001). Indicators of engagement. En L. A. K. Simon, M. Kenny, K. Brabeck, R. M. Lerner (Eds.), Learning to serve: Promoting civil society through service learning. Kluwer.

Illich, I. (1971). Deschooling Society. Harper \& Row. Jacoby, B., \& Associates (2003). Building partnerships for service-learning. Jossey-Bass.

Kendall, J. C. (Ed.) (1990). Combining service and learning: A resource book for community and public service. National Society for Experiential Education.

Liu, G. (1996). Community service in higher education: A decade of development. Providence College.

López-Villarreal, I. (2018). Aprendizaje Servicio en la implementación de política pública para la infancia. RIDAS. Revista Iberoamericana de Aprendizaje Servicio, 5, 1-11.

http://dx.doi.org/10.1344/RIDAS2018.5.1

Lowery, D., May, D. L., Duchane, K. A., Coulter-Kern, R., De' Bryant, Morris, P. V., Pomery, J. G., Bellner, M. (2006). A logic model of service learning: Tensions and issues for further consideration. Michigan Journal of Community Service Learning, 13, 47-60.

https://bit.ly/2QPFMtw

Martín-García, X., Bär-Kwast, B., Gijón-Casares, M., Puig-Rovira, J. M., \& Rubio-Serrano, L. (2021). El mapa de los valores del aprendizaje-servicio. Alteridad, 16(1), 12-22.

https://doi.org/10.17163/alt.v16n1.2021.01

Merriam, S., Cafarella, R., \& Baumgartner, L. (2007). Learning in Adulthood. Jossey-Bass.

Mungaray, A., Ocegueda, J. M., \& Sánchez, M. D. (2002). Retos y perspectivas de la reciprocidad universitaria a través del servicio social en México. ANUES.

ONU, PNUD (2015). Objetivos Desarrollo Sostenible. Agenda 2030. https://bit.ly/3h19Nmh

Pérez-Galván, L. M., \& Ochoa-Cervantes, A. (2017). El aprendizaje-servicio (ApS) como estra- 
tegia para educar en ciudadanía. Alteridad, 12(2), 175-187.

https://doi.org/10.17163/alt.v12n2.2017.04

Pérez, R. (2015). San Lorenzo Datem. En J. Escudero y F. González (Coords.), Solidaridad y voluntariado universitario. Relatos de Experiencias (pp. 33-53). Servicio de Publicaciones (UPSA).

Quiroga, A. (2013). Aprendizaje Servicio en México: participación solidaria en pro de la equidad de género. Revista Internacional de Educación para la Justicia Social (RIEJS), 2(2), 55-73. https://bit.ly/3GbOQ3F

Reardon, K. M. (1998). Participatory action research as service learning. En K.M. Reardon, New Directions for Teaching and Learning (73), 57-64. https://doi.org/10.1002/tl.7307

Rodríguez-Izquierdo, R. M. (2020). Aprendizaje Servicio y compromiso académico en Educación Superior. Revista de Psicodidáctica, $25,45-51$. https://doi.org/10.1016/j.psicod.2019.09.001

Santos-Pastor, M. L., Cañadas, L., Martínez-Muñoz, L. F., Calle-Molina, M. T., Garoz-Puerta, I., Sanz-Arribas, I., Álvarez-Barrio, M. J., \& Ponce-Garzarán, A. (2018). Programa de Educación Física para Jóvenes Universitarios con Discapacidad Intelectual desde Metodología de Aprendizaje-Servicio. En F.J. Murillo Avances en Democracia y Liderazgo Distribuido en Educación. Actas del II Congreso Internacional de Liderazgo y Mejora de la Educación. RILME, 136-139.
Santoyo, D. (2017). Evaluación del Programa de Servicio Social UADY- Peraj "Adopta a un amig@”. Perspectivas Docentes, 64, 9-17. https://doi.org/10.19136/pd.a28n64.1915

Shor, I. (Ed.) (1987). Freire for the classroom: A sourcebook for liberatory teaching. Boynton/Cook Publishers.

Sigmon, R. L. (1979). Service-learning: Three Principles. Synergist. National Center for Service-Learning. Synergist, 8(1), 9-11. https://bit.ly/3EoRiU7

Speck, B. W., \& Hoppe, S. L. (Eds.). (2004). Servicelearning: History, theory, and issues. Greenwood Publishing Group.

Strand, K., Marullo, S., Cutforth, N., Stoecker, R., \& Donohue, P. (2003). Principles of Best Practice for Community-Based Research. Michigan Journal of Community Service Learning, 9(3), 5-15. https://bit.ly/2DrbyKx

Vygotsky, L. S. (1962), Thought and Language. MIT Press.

Weiler, K. (1991). Freire and a feminist pedagogy of difference. Harvard Educational Review, 61, 449-474.

https://doi.org/10.17763/haer.61.4.a102265j168rju84

Zayas, B., Gozálvez, V., \& Gracia, J. (2018). La dimensión ética y ciudadana del Aprendizaje Servicio: Una apuesta por su institucionalización en la Educación Superior. Revista Complutense De Educación, 30(1), 1-15. https://doi.org/10.5209/RCED.55443 


\section{Monitoreo de aprendizajes en escuelas públicas
chilenas en contexto COVID-19}

Dra. María Verónica Leiva-Guerrero es profesora de la Pontificia Universidad Católica de Valparaíso (Chile) (veronica.leiva@pucv.cl) (https:// orcid.org/0000-0002-7641-0087)

(D) Jimena Ivonne Sanhueza-Mansilla es profesora de la Pontificia Universidad Católica de Valparaíso (Chile) (jimena.sanhueza@pucv.cl) (https:// orcid.org/0000-0002-9279-421X)

(D) María Paz Soto-Calderón es profesora de la Pontificia Universidad Católica de Valparaíso (Chile) (maria.soto.c@pucv.cl) (https:// orcid.org/0000-0002-7165-1578)

(D) María Eliana Muñoz-Lameles es profesora de la Pontificia Universidad Católica de Valparaíso (Chile) (maria.munoz.l@pucv.cl) (https:// orcid.org/0000-0002-8784-8535)

Recibido: 2021-02-01 / Revisado: 2021-09-30 / Aceptado: 2021-11-12 / Publicado: 2022-01-01

\section{Resumen}

En el actual contexto de pandemia se ha condicionado la escolaridad, rompiendo con las pautas cotidianas de las escuelas y comprometiendo alternativas paliativas para la puesta en marcha, especialmente las relativas a la evaluación. Las escuelas se ven desafiadas a un seguimiento dimanado del Ministerio de Educación, como la priorización curricular y escenarios de aprendizaje en casa. En este contexto resulta de interés analizar cómo incorporar el proceso de monitoreo y evaluación de aprendizajes en clases no presenciales durante la pandemia, en escuelas públicas de Chile. Para ello, se han seleccionado 50 escuelas públicas que son parte del programa Sumo Primero en Terreno. La metodología de tipo cualitativa se basa en entrevistas en profundidad a los equipos directivos de estas escuelas. Las conclusiones más relevantes son la diversidad de concepciones y finalidades del monitoreo que tienen los directivos, la sistematicidad del mismo en su frecuencia y en las herramientas para llevarlo a cabo, la concentración de la comunicación en el uso de los medios de comunicación WhatsApp, teléfono, mail y redes sociales, así como las dificultades para sistematizar fórmulas que atiendan a la variedad y singularidad de todos y cada uno de sus estudiantes.

Descriptores: Monitoreo de aprendizajes, evaluación formativa, educación a distancia, retroalimentación, COVID-19.

\section{Abstract}

Nowadays, in pandemic context, education has been conditioned to break with the daily school's routine and has engaged to different alternatives to implement changes, especially those related to evaluation. Schools are challenged to follow new guidelines which are issued by the Ministry of Education, such as Priorización Curricular and new learning contexts at home. In this research, it is of a great interest to analyze how to incorporate the process of monitoring and learning assessment in the context of non-face-to-face classes during the pandemic in Chile? This article aim is to analyze the process of monitoring learning in 50 Chilean public schools during covid-19 context. The public schools which have been selected, are part of the Sumo Primero en Terreno program that is implemented by the Ministry of Education (MINEDUC) and the Pontificia Universidad Católica de Valparaíso (PUCV). The methodology is qualitative; interviews were answered by the management teams of these schools. The most relevant conclusions are a wide range of conceptions and monitoring purposes that school leaders have, the systematic nature of the monitoring process, its frequency and the tools to carry it out, the overuse of the multimedia systems and digital gadges (WhatsApp, telephone, mail and social networks), as well as the difficulties that they face to organize different approaches to guarantee learning to each student.

Keywords: Learning monitoring, formative assessment, distance education, feedback, COVID-19.

Forma sugerida de citar: Leiva-Guerrero, M. V., Sanhueza-Mansilla, J. I., Soto-Calderón M. P., \& Muñoz-Lameles, M. E. (2022). Monitoreo de aprendizajes en escuelas públicas chilenas en contexto COVID-19. Alteridad, (17)1, 89-102. https:// doi.org/10.17163/alt.v17n1.2022.07 


\section{Introducción}

El informe "La educación en tiempos de la pandemia de COVID-19" de la CEPAL-UNESCO, es categórico en señalar que la pandemia ha impactado fuertemente en el desarrollo del proceso educativo en casi todas las regiones del mundo, lo cual traerá consecuencias inimaginables, que afectarán principalmente a aquellos estudiantes y familias con menos oportunidades socioeconómicas (MINEDUC, 2020b; Murillo \& Duk, 2020; Rieble \& Viteri, 2020; Zhao, 2020). La pandemia de coronavirus provocó que millones de escuelas cerraran sus puertas forzosamente, durante el 2020, teniendo que reestructurar sus procesos educativos, para así, acoger al alumnado con actividades de aprendizaje remotas o híbridas, mediante dispositivos digitales o material impreso, para dar continuidad al proceso de enseñanza y aprendizaje. Las diferentes soluciones adoptadas para hacer frente al proceso de educación a distancia han dependido de las capacidades y recursos con los que cuenta cada nación (Álvarez et al., 2020; Bos et al., 2020).

Chile, al igual que gran parte de los países del mundo, no se encontraba preparado para brindar una educación virtual, razón por la cual, todos los procesos desplegados han significado grandes desafíos para el sistema educativo, especialmente para los equipos directivos y profesores. No solo tuvieron que diseñar estrategias para organizar el año escolar virtual, sino que además tuvieron que distribuir su liderazgo para propiciar el desarrollo de nuevas capacidades docentes y dar respuesta a las emergentes necesidades escolares y socioemocionales del estudiantado (Harris \& Jones, 2020). Por su parte, los docentes, debieron aprender rápidamente a usar diversas plataformas tecnológicas y modificar sus estrategias pedagógicas y evaluativas para sus clases.

A su vez, varias organizaciones entregaron recomendaciones para apoyar la gestión y docencia en las escuelas, tales como: mejorar el acceso a las tecnologías, desarrollar la colaboración, pro- piciar el bienestar socioemocional, fortalecer el desarrollo profesional docente, crear un ambiente propicio para el aprendizaje y establecer relaciones de confianza entre todos los actores de la comunidad escolar (Propuestas Educación Mesa Social Covid-19, 2020b; Reimers \& Schleicher, 2020, Propuestas Educación trabajo interuniversitario mesa social 3B COVID 19, 2020).

Por otra parte, el Ministerio de Educación de Chile (MINEDUC) desplegó una serie de medidas para continuar con el proceso educativo, entre las que destacan la conectividad, el acceso a dispositivos tecnológicos de estudiantes y la definición de un currículum priorizado que pone énfasis en objetivos imprescindibles que debe alcanzar el estudiantado en cada nivel de enseñanza, para responder a la reducción del año escolar presencial, además de adaptar el currículum al contexto de cada escuela, (MINEDUC, 2020b). Por lo anterior, los equipos directivos se vieron desafiados a establecer nuevas formas de monitoreo para evidenciar fehacientemente cómo y de qué forma está aprendiendo el alumnado. Por ello, este artículo aborda el proceso de monitoreo de aprendizajes en 50 escuelas públicas, en contexto de pandemia, con el objetivo de enriquecer la discusión y reflexión sobre el tema.

\subsection{El monitoreo de aprendizajes en contexto COVID-19}

Los equipos directivos de las escuelas en conjunto con profesores han debido sortear varios retos durante el 2020 y uno de los aspectos claves es el monitoreo en este nuevo escenario, en el cual los y las estudiantes, profesores y profesoras han debido quedarse en sus hogares y desde ahí comenzar a desarrollar la labor educativa. Ulloa y Gajardo (2017) plantean que "El monitoreo se entiende fundamentalmente como la búsqueda de la coherencia entre lo planificado y lo efectivamente enseñado" (p.13), siendo este último aspecto el más difícil de determinar en las escuelas, ya que se requiere de una evaluación permanente (Nercellas, 2016). 
Para Guach y Peña (1995) el monitoreo es:

Un proceso continuo que acompaña y forma parte de la praxis profesional donde tienen lugar la observación, la retroalimentación, el debate crítico y reflexivo sobre el desempeño del docente en el salón de clases y su repercusión en el aprendizaje de sus educandos, así como en la formación y desarrollo de competencias profesionales que tributan a una práctica eficiente. (p. 4)

Por su parte Mokate (2003) establece que "el propósito del monitoreo consiste en detectar de manera oportuna las fortalezas y deficiencias de los procesos de ejecución, para hacer ajustes conducentes a una gestión óptima de las iniciativas" (p. 8). En vista del contexto de pandemia es evidente que estos ajustes son necesarios para enfrentar de mejor manera los cambios en los procesos educativos dada la necesidad de quedarse en los hogares y mantener el proceso educativo funcionando.

El Ministerio de Educación de Chile en su Marco para la Buena Dirección y Liderazgo Escolar establece una dimensión específica para el proceso de monitoreo, señalando que los equipos directivos deben: "Monitorear la implementación integral del currículum y los logros de aprendizaje en todos los ámbitos formativos de los estudiantes para el mejoramiento de los procesos de enseñanza y la gestión pedagógica" (MINEDUC, 2015 p. 25). Esto se torna muy complejo en tiempos de pandemia, debido a que gran parte del alumnado no cuenta con acceso a internet para participar de las clases online, lo que demandó en los equipos directivos y docentes el desarrollo de diferentes formas para llegar a los y las estudiantes, con actividades de aprendizaje para un currículum priorizado y flexible al contexto de las escuelas (MINEDUC, 2016; MINEDUC, 2020a). Sin embargo, la devolución de actividades de aprendizaje por parte de estudiantes es baja, ya sea por problemas de conectividad, por su localización geográfica, estado socioemocional y económico, entre otros, por lo que no se puede evidenciar realmente el aprendizaje del alumnado.

$\mathrm{Al}$ respecto, un estudio realizado por el Ministerio de Educación y el Banco Mundial, en agosto de 2020, establece que solo un $27 \%$ de alumnos y alumnas de escuelas vulnerables tenían cobertura escolar (MINEDUC, 2020b). En esta realidad adquiere relevancia el conocimiento de datos demográficos, por parte de las escuelas para determinar a qué estudiantes se debe entregar mayor apoyo y en qué ámbitos se debe focalizar la ayuda, adaptando de esta forma los procesos educativos al contexto de pandemia e impactar en la mejora educativa (Agencia de la Calidad, 2018a). En este sentido, cobra relevancia el proceso de retroalimentación, ya que los y las estudiantes deben "recibir una retroalimentación oportuna y clara, para definir los aspectos logrados, los errores procedimentales que se encuentren en una tarea y los pasos a seguir para avanzar en el aprendizaje en curso" (Educación 2020, 2020 p. 7).

Estas nuevas estrategias de monitoreo debieron poner énfasis en la diversificación de la enseñanza de todos los y las estudiantes, lo cual fue un gran desafío para el cuerpo docente y equipos directivos, quienes debieron integrar el uso pedagógico de videollamadas, las cuales se realizaban a través de diversas plataformas como Zoom, Google Meet, WhatsApp, Teems, entre otras, adecuando el material educativo e incorporando rúbricas con nuevos espacios para instrucciones, retroalimentaciones y autoevaluación o coevaluación (Gálvez \& Crino, 2020).

\subsection{La evaluación formativa para el monitoreo de aprendizajes}

Uno de los aspectos clave en el nuevo escenario de pandemia para monitorear los aprendizajes es evaluar al alumnado de forma efectiva para evidenciar lo que está aprendiendo y retroalimentarlo oportunamente. Es en este contexto donde cobra relevancia la evaluación formativa, que permite de forma oportuna, y en el pro- 
ceso mismo del acto educativo, fortalecer los aprendizajes de los estudiantes (Hebles et al., 2017; Castillo \& Cabrerizo, 2009; Foster, 2017). Anijovich y Cappelletti (2017) establecen que la evaluación formativa pone en el centro al estudiante y su aprendizaje, para adecuar las prácticas de enseñanza. En Chile la promulgación del Decreto 67 del Ministerio de Educación establece,

Por una parte, fortalecer la integración de la evaluación formativa a la enseñanza para diagnosticar y monitorear de modo más constante y sistemático los aprendizajes de los estudiantes, tomando decisiones pedagógicas de forma oportuna; y, por otra, enriquecer el modo en que se evalúa sumativamente y se califica, para representar y comunicar de mejor manera el aprendizaje y para aportar a motivar y apoyar el aprendizaje de los estudiantes. (MINEDUC, 2018, p. 10)

Lo cierto es que la evaluación está llamada a constituirse en una actividad necesaria para dar cuenta de la calidad del proceso educativo y de los resultados de la enseñanza y el aprendizaje (Förster \& Zepeda, 2017). Para ello, los establecimientos educacionales deben salir de la aplicación, solo, de heteroevaluaciones, con foco en lo sumativo y en la calificación, y avanzar hacia una evaluación participativa, formativa, con presencia de autoevaluaciones para la autorregulación de los aprendizajes desde los propios estudiantes (Anijovich, 2017; Educación 2020, 2020; Mottier, 2010). Además de instalar en las escuelas una cultura evaluativa que implique hacer un giro de paradigma desde la preponderancia de la calificación y evaluaciones sumativas a los procesos de aprendizajes y evaluaciones formativas (Popham, 2013). Al respecto, Anjovich y Capelletti destacan la función pedagógica de la evaluación formativa, dado que "aporta información útil para reorientar la enseñanza (en caso de ser necesario)" (2017, p. 12), invita a la retroalimentación constante y acompañamiento de los estudiantes en la construcción de los aprendizajes esenciales (MINEDUC, 2020a). "Una buena retroalimentación acelera el aprendizaje porque produce motivación y compromiso con el aprendizaje e incentiva el gusto por aprender y mejorar" (MINEDUC, 2020a, p. 5). Por su parte, la UNESCO (2020), refiriéndose a la evaluación para el monitoreo de aprendizajes, señala que se debe tomar en cuenta los aspectos de confinamiento, la ansiedad, las brechas socioeconómicas y educativas, para realizar la retroalimentación al alumnado durante el proceso de aprendizaje.

Por tanto, la flexibilización en una educación remota desde la perspectiva curricular y evaluativa, implica un trabajo articulado e integrado entre directivos, docentes, estudiantes, familias, las asignaturas y lo digital, para fortalecer procesos de responsabilidad y autorregulación en los estudiantes. Así la evaluación, como parte del monitoreo de aprendizajes puede visualizarse como el "proceso que retroalimenta a todos los actores que intervienen en la educación e incluso al sistema educativo como tal" (Escobar, 2014, p. 127)

\section{Método}

El objetivo de esta investigación es describir cómo los equipos directivos de escuelas chilenas abordan el proceso de seguimiento de los aprendizajes en el contexto de pandemia COVID-19, durante el año escolar 2020. Este estudio forma parte de un proyecto mayor denominado Sumo Primero en Terreno que tiene por objetivo instalar competencias para la gestión curricular en equipos directivos de escuelas públicas, ubicadas en las XVI regiones de la organización territorial de Chile. La investigación se realizó con una metodología cualitativa (Flick, 2015).

\subsection{Diseño}

Para la realización de la investigación se utilizó un diseño metodológico descriptivo (Maxwell, 2005), con la aplicación de una entrevista grupal en profundidad (Valles, 1999; Holstein \& Gubrium, 1995), con la finalidad de conocer 
cómo los equipos directivos, compuestos por Jefe/a de Unidad Técnica Pedagógica (cargo de liderazgo pedagógico que en Chile realizan tanto mujeres como hombres, pero que lleva ese nombre) y Director/a de establecimientos educacionales del país, abordan el monitoreo de los aprendizajes del alumnado en pandemia COVID-19. Luego de aplicar las entrevistas y transcribirlas, se asignaron categorías a las respuestas mediante la aplicación ATLAS.ti 9, estas categorías surgieron al agrupar elementos afines que respondieron a los objetivos propuestos para esta investigación, partiendo inicialmente por una codificación abierta para identificar los temas más amplios. A partir de ello, se construyó una codificación axial para reconocer las relaciones más relevantes e identificar los aspectos claves y "críticos" en respuesta a las preguntas que orientaron la investigación. Dos investigadores analizaron el corpus de datos de manera independiente. Una vez acordadas las categorías, dos investigadores codificaron el corpus textual para establecer la fiabilidad.

\subsection{Participantes}

Como población del estudio se contaba con las 200 escuelas públicas chilenas participantes en el Programa Sumo Primero en Terreno en el año 2020 , de las cuales la muestra de tipo no probabilística, intencionada (Latorre et al., 2003) quedó constituida por 50 escuelas, que fueron las que accedieron a participar de la entrevista, cumpliendo con los criterios de categorización insuficiente y medio bajo del establecimiento escolar según la Agencia de la Calidad de la Educación (Agencia de la Calidad de la Educación, 2019), representación geográfica (escuelas ubicadas en las zonas norte, centro y sur del país), participación en entrevista de ambos actores claves de equipo directivo: Director/a y Jefe/a de Unidad Técnica Pedagógica. Los/as participantes previo a la entrevista firmaron un consentimiento informado. En la tabla 1 se caracteriza a las escuelas participantes donde se desempeñan los equipos directivos.

Tabla 1. Caracterización de las cincuenta escuelas públicas categorizadas como insuficiente o medio-bajo, en las que se desempeñan los equipos directivos participantes en la investigación, Chile, 2020

\begin{tabular}{|l|l|l|l|l|l|l|l|}
\hline $\mathbf{N}^{\circ}$ & \multicolumn{1}{|c|}{ Región } & \multicolumn{1}{|c|}{ Comuna } & Categoria & $\mathbf{N}^{\circ}$ & Región & Comuna & Categoría \\
\hline 1 & $\begin{array}{l}\text { Región de } \\
\text { Tarapacá }\end{array}$ & lquique & Medio Bajo & 26 & Región de Valparaíso & Quilpué & Insuficiente \\
\hline 2 & $\begin{array}{l}\text { Región de } \\
\text { Tarapacá }\end{array}$ & lquique & Medio Bajo & 27 & Región de Valparaíso & Quilpué & Medio Bajo \\
\hline 3 & $\begin{array}{l}\text { Región de } \\
\text { Antofagasta }\end{array}$ & Antofagasta & Medio Bajo & 28 & Región de Valparaíso & Valparaíso & Insuficiente \\
\hline 4 & $\begin{array}{l}\text { Región de } \\
\text { Antofagasta }\end{array}$ & Antofagasta & Medio Bajo & 29 & Región de O'Higgins & Rancagua & Medio Bajo \\
\hline 5 & $\begin{array}{l}\text { Región de } \\
\text { Antofagasta }\end{array}$ & Antofagasta & Medio Bajo & 30 & Región del Maule & San Clemente & Insuficiente \\
\hline 6 & $\begin{array}{l}\text { Región de } \\
\text { Antofagasta }\end{array}$ & Antofagasta & Insuficiente & 31 & Región del Maule & Villa Alegre & Insuficiente \\
\hline 7 & $\begin{array}{l}\text { Región de } \\
\text { Atacama }\end{array}$ & Chañaral & Medio Bajo & 32 & Región del Maule & Talca & Medio Bajo \\
\hline 8 & $\begin{array}{l}\text { Región de } \\
\text { Atacama }\end{array}$ & Freirina & Medio Bajo & 33 & Región del Nuble & Chillán & Medio Bajo \\
\hline
\end{tabular}




\begin{tabular}{|c|c|c|c|c|c|c|c|}
\hline $\mathbf{N}^{\circ}$ & Región & Comuna & Categoria & $\mathbf{N}^{\circ}$ & Región & Comuna & Categoría \\
\hline 9 & $\begin{array}{l}\text { Región de } \\
\text { Atacama }\end{array}$ & Chañaral & Medio Bajo & 34 & Región del Ñuble & Pinto & Medio Bajo \\
\hline 10 & $\begin{array}{l}\text { Región de } \\
\text { Coquimbo }\end{array}$ & La Serena & Medio Bajo & 35 & Región del Bío Bío & Santa Bárbara & Medio Bajo \\
\hline 11 & $\begin{array}{l}\text { Región de } \\
\text { Coquimbo }\end{array}$ & La Serena & Medio Bajo & 36 & Región del Bío Bío & Lebu & Medio Bajo \\
\hline 12 & $\begin{array}{l}\text { Región de } \\
\text { Coquimbo }\end{array}$ & La Serena & Medio Bajo & 37 & Región de Aysén & Coyhaique & Medio Bajo \\
\hline 13 & $\begin{array}{l}\text { Región de } \\
\text { Coquimbo }\end{array}$ & La Higuera & Medio Bajo & 38 & Región de Aysén & Coyhaique & Medio Bajo \\
\hline 14 & $\begin{array}{l}\text { Región de } \\
\text { Coquimbo }\end{array}$ & Vicuña & Medio Bajo & 39 & Región de Aysén & Aysén & Medio Bajo \\
\hline 15 & $\begin{array}{l}\text { Región de } \\
\text { Coquimbo }\end{array}$ & Ovalle & Medio bajo & 40 & Región Metropolitana & Macul & Medio Bajo \\
\hline 16 & $\begin{array}{l}\text { Región de } \\
\text { Coquimbo }\end{array}$ & Ovalle & Medio bajo & 41 & Región Metropolitana & La cisterna & Insuficiente \\
\hline 17 & $\begin{array}{l}\text { Región de } \\
\text { Coquimbo }\end{array}$ & Punitaqui & Insuficiente & 42 & Región Metropolitana & Lo Prado & Insuficiente \\
\hline 18 & $\begin{array}{l}\text { Región de } \\
\text { Coquimbo }\end{array}$ & Illapel & Insuficiente & 43 & Región Metropolitana & $\begin{array}{l}\text { San José de } \\
\text { Maipo }\end{array}$ & Medio Bajo \\
\hline 19 & $\begin{array}{l}\text { Región de } \\
\text { Coquimbo }\end{array}$ & Illapel & Medio Bajo & 44 & Región Metropolitana & El Bosque & Medio Bajo \\
\hline 20 & $\begin{array}{l}\text { Región de } \\
\text { Coquimbo }\end{array}$ & Salamanca & Medio Bajo & 45 & Región Metropolitana & Peñaflor & Medio Bajo \\
\hline 21 & $\begin{array}{l}\text { Región de } \\
\text { Coquimbo }\end{array}$ & Monte Patria & Medio Bajo & 46 & Región Metropolitana & Peñaflor & Insuficiente \\
\hline 22 & $\begin{array}{l}\text { Región de } \\
\text { Coquimbo }\end{array}$ & Coquimbo & Medio Bajo & 47 & Región Metropolitana & Peñaflor & Medio Bajo \\
\hline 23 & $\begin{array}{l}\text { Región de } \\
\text { Valparaíso }\end{array}$ & San Esteban & Medio Bajo & 48 & Región Metropolitana & Peñaflor & Insuficiente \\
\hline 24 & $\begin{array}{l}\text { Región de } \\
\text { Valparaíso }\end{array}$ & Quilpué & Medio Bajo & 49 & Región Metropolitana & La Florida & Medio Bajo \\
\hline 25 & $\begin{array}{l}\text { Región de } \\
\text { Valparaíso }\end{array}$ & Panquehue & Medio Bajo & 50 & Región Metropolitana & Talagante & Medio Bajo \\
\hline
\end{tabular}

Fuente: Sistema de Gestión Programa Sumo Primero en Terreno. https://bit.ly/3r8hKxB 


\subsection{Instrumento}

Para la investigación se aplicó una entrevista en profundidad en modalidad virtual, a través de la plataforma Google Meet, la cual fue grabada con el sistema de la herramienta. Dicha entrevista se realizó en una sesión de una hora y treinta minutos, usando la técnica activa-reflexiva (Valles, 1999; Hostein \& Gubrium, 1995). Esto implica una interacción en la cual los partícipes, entrevistado/da y entrevistador/ra son asumidos como personas que interactúan abiertamente, guiados por preguntas flexibles de conversación. Las preguntas planteadas fueron: ¿Cómo comprenden el monitoreo de aprendizajes en contexto de pandemia?, ¿Cómo y con qué frecuencia implementan el monitoreo de aprendizajes en la escuela? y ¿Cuáles son las dificultades para implementar el monitoreo en contexto de pandemia en la escuela? La definición de las preguntas fue consensuada por el equipo de investigadores en función de la pregunta de investigación del estudio sobre ¿cómo los equipos directivos abordan el monitoreo de aprendizajes en situación de crisis sanitaria por COVID-19? Todas las entrevistas fueron grabadas y transcritas de manera íntegra para su posterior procesamiento y análisis. Para la transcripción y registro de información se siguieron las recomendaciones de MacLellan et al. (2003).

La información se procesó a través de un análisis de contenido de tipo temático (Valles, 1999; Bardin, 1986). Se consideraron tanto categorías deductivas como inductivas. Las primeras se derivaron de la literatura revisada. Las categorías inductivas consideran los descubrimientos hallados (Mayring, 2000). Debido al volumen de información que implicó las respuestas de las 50 entrevistas, se construyó un libro de códigos (MacQueen et al., 1998) que orientó el trabajo grupal de análisis, donde investigadores analizaron el corpus de datos de manera independiente, para posteriormente consensuar las categorías levantadas y proceder a la codificación textual.
Para realizar el análisis de datos cualitativos se utilizó el software Atlas.ti (Friese, 2017).

\section{Resultados y discusión}

\subsection{Monitoreo de aprendizajes de directivos en contexto de pandemia}

Con relación a cómo comprenden el monitoreo en contexto de pandemia los directores/as y jefes/ as de UTP de las escuelas, señalan principalmente que es un proceso relevante de información del y sobre el aprendizaje de los estudiantes, que se recoge en el proceso educativo, introduciendo retroalimentaciones y los ajustes necesarios para la mejora de los aprendizajes. Además, implica la reflexión y toma de decisiones como consecuencia de la información detectada en este proceso denominado monitoreo.

El monitoreo se comprende y se visualiza en las distintas etapas de desarrollo de nuestros estudiantes y en los diferentes niveles de aprendizaje posible de ser desarrollado por la vía del trabajo a distancia. El monitoreo es relevante, y se realiza a través de recursos digitales. El monitoreo del proceso de aprendizaje y la evaluación de logros, desde una perspectiva formativa, permite ir realizando ajustes, obtener información actualizada e ir retroalimentando a los estudiantes. (ED897)

El monitoreo es muy importante, ya que a través de esta instancia se puede recolectar información que permitirá analizar y utilizar evidencias para hacer un seguimiento al progreso de cada uno de los estudiantes, también incide en el resultado de los aprendizajes, en la mejora permanente de los procesos de enseñanza aprendizaje y para evidenciar los grados de avances y déficit, de esta manera realizar la retroalimentación de forma inmediata. La evaluación formal está concentrada en los logros esperados y alcanzados por los estudiantes. Esta debe proporcionar una evidencia que sea creable y útil para ser usados en el futuro 
para la toma de decisiones con los educandos. Permite al docente perfeccionar su propia práctica. (ED1245)

No obstante, otros directivos lo visualizan como un proceso complejo en el contexto de clases remotas, burocrático con orientación a la supervisión y con la única finalidad de establecer el cumplimiento de la cobertura curricular.

El monitoreo es un proceso continuo, permanente y sistemático que se da de manera paralela al proceso enseñanza-aprendizaje, al realizar una supervisión y seguimiento sobre la cobertura curricular. (ED1893)

Se comprende como una barrera más dentro del difícil escenario que desarrollan los docentes. Por un lado, se piensa que el registro de evidencias es algo engorroso (planillas de registro) y por otro, la evaluación aparece como una de las grandes complicaciones del proceso, considerando para ello que la evaluación se debe realizar de manera sincrónica (para lo cual no existen los recursos de parte del sostenedor). (ED489)

Se comprende el monitoreo y la evaluación del aprendizaje como un proceso complejo dentro de los nuevos lineamientos y el proceso que implica la educación a distancia, considerando que no existe el contacto físico y la interacción que debiera darse entre docente y estudiante. (ED10753)

Al respecto, observamos dos miradas por parte de los equipos directivos sobre el monitoreo de aprendizajes en pandemia, algunos lo entienden desde una perspectiva más cuantitativa, poniendo acento en la cantidad del aprendizaje logrado, sin contemplar el cómo y el para qué fue logrado dicho aprendizaje, y otros, más cualitativamente, al poner énfasis en los avances y logros de una manera mucho más descriptiva, considerando las características de los y las estudiantes, sus diferencias individuales y consultando los resultados en la toma de decisiones pedagógicas. Además, se aprecia una tensión, entendiendo el monitoreo centrado en la acción docente como el proceso que da cuenta del cumplimiento del currículum o como una supervisión de tareas y/o actividades desempeñadas por los y las estudiantes, razón por la cual, los equipos directivos requieren asegurar una planificación - con tiempo y materiales - para la consecución de metas que deben monitorear, acompañar y retroalimentar con el fin de comprender y explicar los resultados de aprendizaje de sus estudiantes (Volante et al., 2015).

En la Figura 1 se observan las categorías generadas a partir de las respuestas de los equipos directivos sobre la comprensión del monitoreo de aprendizajes en pandemia COVID-19.

Figura 1. Categorías halladas sobre cómo comprenden el monitoreo, jefe de UTP y directores/as de 50 escuelas públicas de Chile, 2020

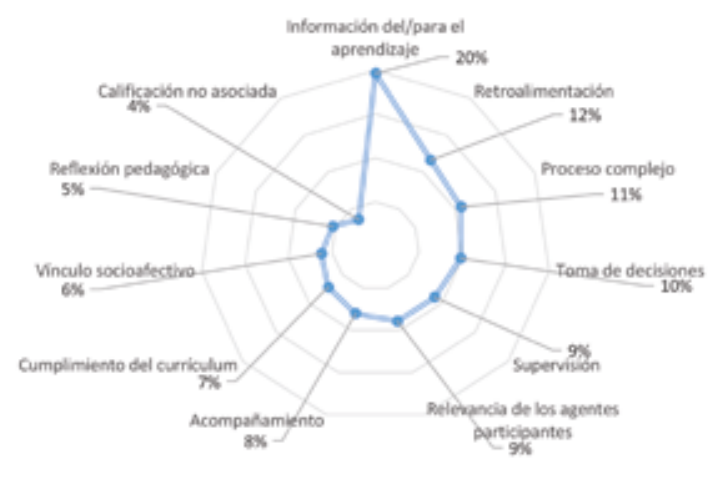


Finalmente, se señala como elementos emergentes, la comprensión de retroalimentación, acompañamiento y vínculo socioafectivo, los cuales están relacionados y son clave para que el monitoreo que se lleve a cabo junto a la participación de la familia/apoderados, como la de los/ las estudiantes; donde en este caso se señala que en ocasiones es escasa. $\mathrm{Al}$ respecto, Educación 2020 (2020), plantea que es necesario retroalimentar constantemente a los y las estudiantes, entregando una definición clara de los que se va a evaluar y cómo se realizará este proceso, generando conversaciones y acuerdos con el alumnado, haciendo llamadas o videollamadas, promoviendo la autoevaluación o la coevaluación.

\subsection{Cómo y con qué frecuencia implementan el monitoreo de aprendizajes los equipos directivos}

Respecto de la implementación del monitoreo, se evidencia en el discurso de los directivos elementos vinculados a recursos pedagógicos como medios de comunicación; entendiendo que la educación a distancia marcó el escenario pedagógico. De los recursos pedagógicos más utili- zados fueron las guías, trabajos y/o tareas (66 $\%)$ como estrategias para evidenciar desempeños que permitan realizar un monitoreo de los aprendizajes. Posteriormente, se encuentran las subcategorías pautas $(13 \%)$ como recurso para sistematizar lo realizado por los/las estudiantes, siendo estas para indicar la presencia o ausencia de una acción de forma dicotómica o con un nivel mayor de profundidad que permitiese dar cuenta de un descriptor o nivel de desempeño de una tarea determinada. Seguido se encuentra la observación directa en clases sincrónicas (9\%), lo que es variable, pues las adaptaciones, tanto de la docencia como de los y las estudiantes para comprender este sistema nuevo como ambiente de aprendizaje, indica en mayor o menor media la adaptación que tienen los mismos para implementar el monitoreo. Además de contar con los recursos de conectividad y tecnológicos de forma irregular. Finalmente, la subcategoría preguntas $(6 \%)$ y formularios $(6 \%)$ se enfoca en recursos pedagógicos que se utilizan en algún momento de la clase sincrónica o de forma asincrónica para conocer el nivel de comprensión de algún objetivo de aprendizaje trabajado y/o existencia de inquietudes, dudas, entre otro tipo de información relevante para el logro del aprendizaje (ver Figura 2).

Figura 2. Estrategias de cómo implementan el monitoreo de aprendizajes 50 escuelas públicas chilenas, 2020

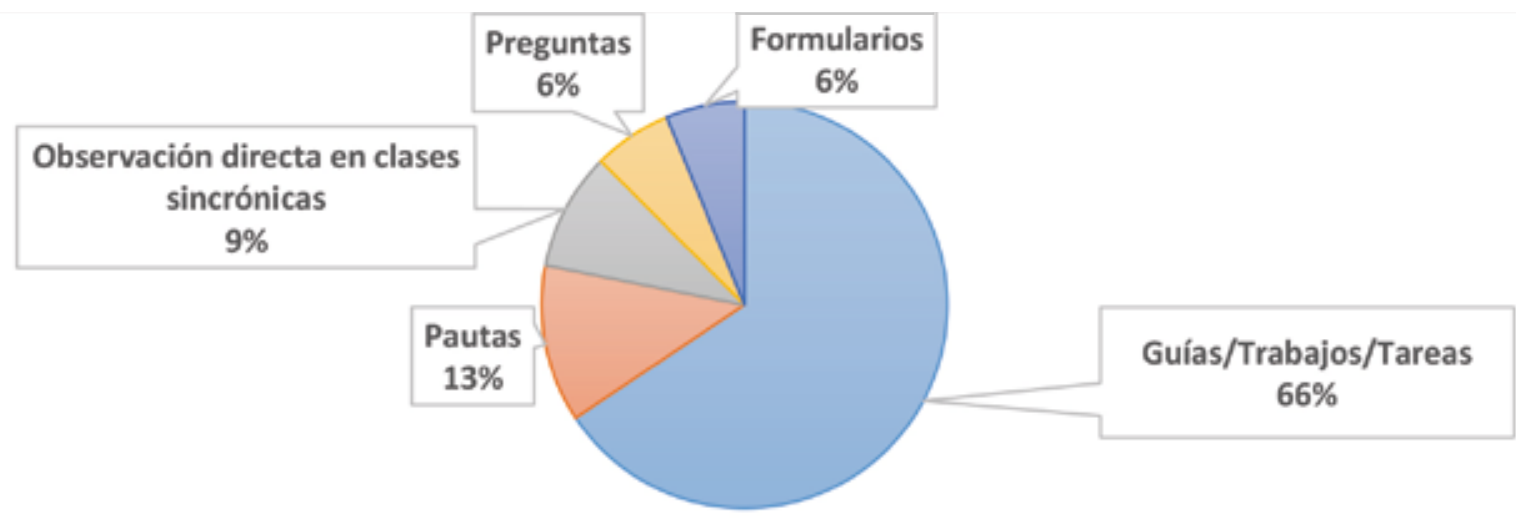


Los medios de comunicación más nombrados por los equipos directivos utilizados en la implementación del monitoreo responden al uso de la aplicación WhatsApp (32\%) dada su versatilidad como canal de comunicación; ya sea por permitir el envío de mensajes escritos, audios, imágenes, videos y/o videollamadas. Seguido de la llamada telefónica (25\%), plataformas virtuales y/o redes sociales $(18 \%)$, videollamadas $(14 \%)$ y correo electrónico (11\%). Se aprecia que la gran mayoría de los medios señalados son de carácter virtual y, por lo tanto, requieren de una conexión a internet dependiendo de la compañía de telecomunicaciones que libera datos para el uso de ciertas aplicaciones y redes sociales.

Lo anterior, es coincidente con lo planteado por el MIDE UC, de retroalimentar y monitorear los aprendizajes por todos los medios disponibles: llamadas, pequeños videos, mensajes de texto, WhatsApp, comentarios escritos, y con los estudiantes que más requieren ayuda, retroalimentarlos directamente a través de llamadas para así tener la certeza que han entendido las instrucciones y poder aclarar sus dudas inmediatamente (MIDE UC, 2020).

Sin lugar a dudas, monitorear en un contexto de clases no presencial implica innovar en las estrategias evaluativas virtuales donde el estudiantado debe ser el protagonista en la valoración de sus aprendizajes, sin embargo, desde la virtualidad la evaluación sigue siendo una tarea pendiente y con insuficiente desarrollo en los docentes (Maureira et al., 2020).

Sobre la frecuencia que se implementa el monitoreo de aprendizajes en las escuelas, mayoritariamente $(28 \%)$ se haría diaria y $(28$ $\%)$ quincenalmente, semanal (16\%) y en menor medida (4 \%) mensualmente. Llama la atención que los directivos de algunas escuelas no lograron especificar con claridad la frecuencia (24\%), señalando que era relativo, porque dependía de cada docente, lo que evidencia que no existiría una definición institucional, sobre los tiempos en que se debe realizar dicho proceso.

Nos falta concretar un lineamiento institucional, sobre cómo y cuándo monitorear los aprendizajes de los estudiantes en el difícil escenario de clases no presenciales por el Covid. (ED8348)

No se debe olvidar que el proceso de monitoreo permite "sentar las bases para una toma de decisiones pedagógicas respecto de los procesos de enseñanza y aprendizaje" (MINEDUC, 2016, p. 3).

\subsection{Dificultades para implemen- tar el monitoreo en contexto de pandemia}

Un $67 \%$ de los equipos directivos señalan que la principal dificultad para implementar el monitoreo en contexto de pandemia son las condiciones educativas diversas que ha generado la propia pandemia, por las distintas modalidades sincrónicas y asincrónicas que se han implementado las clases, la localización geográfica de las escuelas, el ambiente emocional y de incertidumbre que viven los niños/ niñas y sus familias. Otra dificultad (33\%) derivada del uso de recursos digitales y virtuales; que permiten mantener la comunicación sincrónica o asincrónica con el estudiantado.

Presencia de esta crisis sanitaria se nos presenta como un proceso difícil que nos permite observar la participación y el compañerismo de los estudiantes que viven un período adverso, donde es más relevante que el currículum implementado. (ED114)

Contexto de pandemia ha resultado difícil un mejor desarrollo de este tipo de evaluación. (ED4356)

Los medios con los cuales se hace el trabajo remoto no han ayudado. (ED306) 
En pandemia la dificultad de monitorear los aprendizajes sistemáticamente ha sido contar con recursos digitales e insumos para abordar el estado emocional de los alumnos, producto de la propia pandemia. (ED2119)

No contamos con las herramientas digitales que nos permitan realizar estos procesos debido a los problemas de conectividad de los estudiantes $\mathrm{y}$ sus familias. Algunos profesores han podido monitorear y aplicar pequeñas evaluaciones formativas, pero no en todos los casos, ni todos los profesores de asignaturas, ya que el trabajo remoto lo lideran los profesores jefes. (ED11704)

En complemento a los hallazgos expuestos, Murillo y Duck (2020), plantean que las dificultades para el proceso educativo en pandemia, más allá de los problemas de conectividad, está el manejo del mundo digital. Las escuelas de América Latina en un alto porcentaje, no cuentan con las condiciones y competencias para asumir el tremendo desafío de una educación remota.

Pero, por otra parte, es fundamental, en estos contextos de crisis que implican una educación a distancia, incorporar a otros agentes evaluativos como son los estudiantes y las familias, a través del fortalecimiento y toma de consciencia de la autoevaluación y vínculo con las familias que son las que observan el desempeño de los y las estudiantes en los hogares. Al respecto, Aguilar-Gordón (2020), señala que uno de los desafíos para el cumplimiento de la función de la familia en el proceso de aprendizaje:

Es la comunicación permanente para potenciar la participación, la colaboración, la solución de problemas, el uso equilibrado de redes sociales y demás insumos tecnológicos, la emisión de ideas, sentimientos y pensamientos conforme a necesidades e intereses de los niños y jóvenes. (p. 222)

Finalmente, las dificultades para poder monitorear lo que realmente han aprendido los/ las estudiantes en un contexto de pandemia, que implicó un proceso de enseñanza y aprendizaje sincrónico y asincrónico, no presencial, obliga a repensar y profundizar prácticas evaluativas más cualitativas, donde el alumnado debe asumir una mayor autonomía frente a ellas, a través de instrumentos que inviten a la reflexión y autorregulación de su aprendizaje como autoevaluaciones y rúbricas. Así, la evaluación no se concentra exclusivamente en el profesorado, sino también en las y los estudiantes que se involucran en el proceso y establecen valoraciones acerca de su propio desempeño (Moreno, 2021).

\section{Conclusiones}

El monitoreo de los aprendizajes es una condición básica y necesaria en todo contexto educativo, instituyéndose como un principio pedagógico insoslayable que debe tener su aplicación didáctica y práctica en cada situación de enseñanza-aprendizaje. En las condiciones actuales de pandemia sufridas desde el año 2020, que ha llevado a la dispersión de los contextos didáctico-pedagógicos, con las aulas cerradas y un uso asistémico de metodologías alternativas, especialmente aquellas que pudieron ser implementadas por los sistemas virtuales, con desiguales efectos, por razones de condición social, disponibilidad de recursos, condiciones socioeconómicas de las familias, diseminación geográfica y distancia de las escuelas, ha puesto en una situación muy compleja la aplicación de los sistemas de interacción ordinarios. Así, tanto por la gestión educativa, como por parte de las propias escuelas. Por tanto, cabe buscar alternativas para la interacción educativa desde el proceso analizado de monitoreo de los aprendizajes, que lógicamente requiere una gran variedad de iniciativas, tanto por su diversidad de acuerdo con las necesidades concretas de cada estudiante, como por su intensidad, precisamente por las circunstancias personales de cada estudiante.

Sin embargo, a la hora de conceptualizar este proceso por parte de los directivos se da una gran diversidad de concepciones, casi todas ellas asociadas a los aspectos de supervisión, informa- 
ción, animación y/o rendición de cuentas, siendo solamente que la quinta parte centra tal acción en torno a aprendizaje, como mecanismo para guiarlo.

No obstante, se releva la consideración del uso de herramientas ad hoc para llevar a cabo tal tarea, mostrándose que en las dos terceras parte de los casos se utilizan guías, trabajos o tareas como elementos que facilitan realizar un seguimiento de lo realizado. Sin embargo, la frecuencia con la que se hacen tales seguimientos resulta ser muy asistemática, con frecuencias no definidas y en las que apenas una cuarta parte lo refieren a diaria o semanalmente y en algunos casos mensualmente, por lo que la mayoría no reporta con precisión que sistematicidad temporal se tiene establecida para tal tarea, considerando en consecuencia que no se ha previsto, o no se tiene constancia de la misma.

Los mecanismos para implementarla, considerando que se basa en un proceso de comunicación, donde utilizan los medios más habituales, caso del WhatsApp, teléfono, redes sociales y correo electrónico, por lo que se puede considerar que son medios que aseguran la bidireccionalidad de la información, siempre en la consideración que habrá casos en los que tales sistemas no los tienen a su disposición, caso de zonas rurales alejadas o familias con escasos recursos para el acceso a las redes.

En síntesis, se puede señalar que a las condiciones restrictivas y condicionantes que genera la propia pandemia, se añaden ahora otras derivadas de las infraestructuras del sistema escolar, la propia formación del profesorado, el sistema de gestión u organización del currículum y la diversidad de situaciones a las que este ha de acomodarse, bien fuera por las particularidades de los y las estudiantes, como por la diversidad de situaciones sociofamiliares-económicas y geográficas del estudiantado y sus familias, pero también por las propias dinámicas institucionales de las escuelas en las que se hallan implicados profesores y directivos.

\section{Limitación y prospectiva}

Las principales limitaciones del estudio se refieren a la cantidad de escuelas analizadas, en total $50 \mathrm{y}$ con ello los resultados de una muestra acotada del total de escuelas participantes del programa Sumo Primero en Terreno. Por otra parte, la modalidad virtual de realización de las entrevistas, por el contexto de pandemia COVID-19, pudieron limitar una mayor profundización y acercamiento en las respuestas a las preguntas sobre el monitoreo de aprendizajes. Desde una mirada prospectiva, el artículo entrega hallazgos relevantes sobre el monitoreo de aprendizajes en escuelas públicas ubicadas en diferentes regiones del país, que sería interesante contrastar con establecimientos escolares de otras dependencias, con procedimientos de recolección de información presenciales.

\section{Agradecimiento}

Las autoras agradecen al Programa Sumo Primero en Terreno del Ministerio de Educación de Chile, a la Pontificia Universidad Católica de Valparaíso y a las escuelas participantes en el programa.

\section{Apoyos y soporte financiero de la investigación}

Programa Sumo Primero en Terreno del Ministerio de Educación de Chile

\section{Referencias bibliográficas}

Aguilar-Gordón, F. (2020). Del aprendizaje en escenarios presenciales al aprendizaje virtual en tiempos de pandemia. Estudios pedagógicos (Valdivia), 46(3), 213-223.

https://dx.doi.org/10.4067/S0718-07052020000300213

Agencia de la Calidad de la Educación (2018a). Guía metodológica para el uso de datos.

https://bit.ly/30Fp0pQ

Agencia de la Calidad de la Educación (2018b). Nuevo Sistema Nacional de Evaluación de Aprendizajes. La evaluación al servicio de los aprendizajes. https://bit.ly/30KWHpU

Agencia de la Calidad de la Educación (2019). Categoría de desempeño de los centros escolares. https://bit.ly/3CyeRrG

Álvarez, H., Arias, E., Bergamaschi, A., López, A., Noli, A., Ortiz, M., Pérez, M., Rieble, S., 
Rivera, M., Scannone, R., Vásquez, M., \& Viteri, A. (2020). La educación en tiempos del coronavirus: Los sistemas educativos de América Latina y el Caribe ante COVID-19. [Education in times of Coronavirus: Latin America and the Caribbean's education systems in the face of COVID-19]. Banco Interamericano de Desarrollo.

https://dx.doi.org/10.18235/0002337

Anijovic, R. \& Cappelletti, G. (2017). La evaluación como oportunidad. Paidós.

Anijovich, R. (2020, 28 de mayo). "Evaluar sí, pero qué y cómo" [video]. YouTube.

https://youtu.be/araSxpBTlGs

Bardin, L. (1986). El análisis de contenido. Akal.

Bos, M., Minoja, L., \& Dalaison, W. (2020). Estrategias de reapertura de escuelas durante covid19. Banco Interamericano de Desarrollo. [Strategies for school reopenings during the COVID-19 pandemic].

https://doi.org/10.18235/0002334

Castillo, S., \& Cabrerizo, J. (2009) Evaluación educativa de aprendizajes y competencias. Pearson.

CEPAL/UNESCO (Comisión Económica para América Latina y el Caribe/Organización de las Naciones Unidas para la Educación, la Ciencia y la Cultura). (2020). "La educación en tiempos de la pandemia de COVID 19", Informe COVID-19 CEPAL-UNESCO, Santiago, agosto. https://bit.ly/3Hz8Sqx

Educación 2020 (2020). Educar en tiempos de pandemia: Parte 3. Recomendaciones pedagógicas para evaluar aprendizajes en tiempos de Covid-19. https://bit.ly/3qU8Nrp

Escobar, G. (2014). La evaluación del aprendizaje, su evolución y elementos en el marco de la formación integral. Revista de Investigaciones UCM, 14(24), 126-141.

https://bit.ly/3CsWWCQ

Foster, C. (2017). El poder de la evaluación en el aula: mejores decisiones para promover aprendizajes. Ediciones UC

Förster, C., \& Zepeda, S. (2017) Planificando integradamente la enseñanza y la evaluación. En C. Förster (Ed.), El poder de la evaluación en el aula (pp.74-93). Ediciones UC.

Friese, S. (2017). ATLAS.ti 8 Windows User Manual. Berlin: ATLAS.ti Scientific Software Development GmbH. https://bit.ly/3x397VI
Gálvez-Donoso, V., \& Crino-Ortiz, F. (2020). La evaluación escolar en tiempos de pandemia. Revista de psicopedagogía, 163, 29-47.

https://bit.ly/3CDR0qt

Guach-Castillo, J., \& Peña-Gálvez, R. (1995). El método de seguimiento o monitoreo. Una experiencia en la formación reflexiva-creativa del programa PRYCREA. Revista Crecemos Internacional, 2. https://bit.ly/3HARly7

Harris, A., \& Jones, M. (2020). COVID 19 - liderazgo escolar en tiempos disruptivos. [COVID-19school leadership in disruptive times]. School Leadership \& Management, 40(4), 243-247. https://doi.org/10.1080/13632434.2020.1811479

Hebles, M., Dos Santos, M., Alvarez de Eulate, C., \& Villardon, L. (2017). Diseño y validación de la Escala Evaluación de los Aprendizajes. [Design and validation of the Assessment of Learning Scale (EEA)]. Revista de currículum y formación del profesorado, 21(2), 107-126. https://bit.ly/3Hxkg6d

Holstein, J. A., \& Gubrium, J. F. (1995). Qualitative research methods, Vol. 37. The active interview. Sage Publications, Inc. https://doi.org/10.4135/9781412986120

Latorre, A., Rincón, D., \& Arnal, J. (2003). Bases metodológicas de la investigación educativa. Grao

MacQueen, K. M., McLellan, E., Kay, K., \& Milstein, B. (1998). Codebook development for team-based qualitative analysis. Cultural Anthropology Methods, 10, 31-36.

https://doi.org/10.4236/psych.2011.24050

Maureira, O., Vásquez, M., Garrido, F., \& Olivares, M. (2020). Evaluación y coevaluación de aprendizajes en blended learning en educación. Revista Alteridad, 15(2), 190-203.

https://doi.org/10.17163/alt.v15n2.2020.04

Maxwell, J. (2005). Diseño de investigación cualitativa: un enfoque interactivo. Sage.

Mayring, P. (2000). Qualitative Content Analysis. Forum Qualitative Sozialforschung. Forum: Qualitative Social Research, 1(2).

http://dx.doi.org/10.17169/fqs-1.2.1089

MIDE UC. (2020, 10 de septiembre). Retroalimentación: el corazón de la evaluación para el aprendizaje [video]. YouTube. https://bit.ly/3FuZSke

Ministerio de Educación de Chile (MINEDUC) (2015). Marco para la Buena Dirección y Liderazgo Escolar. Santiago de Chile. 
https://bit.ly/3x5UQaR

Ministerio de Educación de Chile (MINEDUC) (2016). Monitoreando la implementación curricular y los logros de aprendizaje. Santiago de Chile. https://bit.ly/3CungfH

Ministerio de Educación de Chile (MINEDUC. (2018). Unidad de Currículum y evaluación. Orientaciones para la implementación del Decreto 67. https://bit.ly/3FApMDo

Ministerio de Educación de Chile (MINEDUC) (2020a). Orientaciones para la implementación de la Priorización Curricular en forma remota y presencial. Santiago de Chile. https://bit.ly/3kVtmQx

Ministerio de Educación de Chile (MINEDUC), Centro de Estudios (2020b). Impacto del COVID-19 en los resultados de aprendizaje y escolaridad en Chile. Santiago, Chile.

https://bit.ly/3CyByft

Mokate, K. (2003). Convirtiendo el "monstruo" en aliado: la evaluación como herramienta de la gerencia social.Banco Interamericano de Desarrollo. https://bit.ly/30EALNh

Moreno, T. (2021). Cambiar la evaluación: un imperativo en tiempos de incertidumbre. Revista Alteridad, 16(2), 223-234

https://doi.org/10.17163/alt.v16n2.2021.05

Mottier, L. (2010). Evaluación formativa de los aprendizajes: síntesis crítica de los trabajos francófonos. En R. Anijovich (Ed.), La evaluación significativa (pp. 43-71). Editorial Paidós

Murillo, J., \& Duk, C. (2020). El Covid-19 y las Brechas Educativas. [The Covid-19 and the Educational Gaps]. Revista latinoamericana de educación inclusiva, 14(1), 11-13.

https://dx.doi.org/10.4067/S0718-73782020000100011

McLellan, E., MacQueen, K., \& Neidig, J. (2003). Beyond the Qualitative Interview: Data Preparation and Transcription. Field Methods, 15(1), 63-84.

https://doi.org/10.1177/1525822X02239573

Nercellas, M. (2016). La noción de cobertura curricular y su impacto en la visión del aprendizaje y de la evaluación. https://bit.ly/30F6A8j

Rieble-Aubourg, S., \& Viteri, A. (2020). Educación más allá del COVID-19. Banco Interamericano de Desarrollo. http://dx.doi.org/10.18235/0002654
Popham, J. (2013). Evaluación trans-formativa: el poder transformador de la evaluación formativa. Narcea.

Propuestas Educación Mesa Social Covid-19 (2020a). Didácticas para la proximidad: aprendiendo en tiempos de crisis. Santiago de Chile. https://bit.ly/30PSj8W

Propuestas Educación Mesa Social Covid-19 (2020b). Liderazgo escolar: aprendiendo en tiempos de crisis. Santiago de Chile.

https://bit.ly/3oMi4ip

Propuestas Educación Trabajo Interuniversitario Mesa Social 3B COVID-19. (2020). Pontificia Universidad Católica de Chile y Universidad de Chile. https://bit.ly/3ctOmce

Reimers, F., \& Schleicher, A. (2020). Aprendiendo durante la pandemia: de la disrupción a la innovación. https://bit.ly/3kSXJab

Sumo Primero en Terreno (2020). Guion Taller $N^{\circ} 8$. Evaluando para el monitoreo de los aprendizajes. Liderazgo escolar.

https://bit.ly/3Cuohol

Ulloa, J., \& Gajardo, J. (2017). Gestión de la implementación curricular. Informe Técnico $\mathrm{N}^{\circ} 5$. Líderes Educativos, Centro de Liderazgo para la Mejora Escolar. Chile.

https://bit.ly/32jVOFh

UNESCO (2020). Experiencias de evaluación formativa entre miembros de comunidades educativas latinoamericanas: caracterización de los resultados de encuesta de la UNESCO sobre evaluación formativa. [Formative assessment experiences among members of Latin American educational communities]. https://bit.ly/3p2PABt

Valles, M. (1999). Técnicas cualitativas de investigación social. Reflexión metodológica y práctica profesional. Síntesis.

Volante, P., Bogolasky, F., Derby, F., \& Gutiérrez, G. (2015). Hacia una teoría de acción en gestión curricular: Estudio de caso de enseñanza secundaria en matemática. Psicoperspectivas, 14(2), 96-108.

https://dx.doi.org/10.5027/PSICOPERSPECTIVAS-VOL14ISSUE2-FULLTEXT-445

Zhao, Y. (2020). COVID-19 como catalizador del cambio educativo. [COVID-19 as a catalyst for educational change]. Perspectivas, 49, 29-33.

https://doi.org/10.1007/s11125-020-09477-y 


\section{ALTERIDAD \\ Interacción, diálogo y prácticas pedagógicas en el bachillerato \\ Interaction, dialogue and pedagogical practices in high school}

(1) Rita de Cássia de Almeida Rezende es profesora en el Distrito Federal (Brasil) (ritinhademaria@gmail.com) (https://orcid.org/0000-0002-3354-7972)

(1) Dr. Geraldo Caliman es coordinador de la Cátedra UNESCO de Juventud, Educación y Sociedad en la Universidad Católica de Brasilia (Brasil) (caliman@p.ucb.br) (http://orcid.org/0000-0003-2051-9646)

Recibido: 2021-05-03 / Revisado: 2021-09-01 / Aceptado: 2021-10-25 / Publicado: 2022-01-01

\section{Resumen}

La sociedad demanda habilidades de autonomía, dinamismo y protagonismo frente a los más diversos desafíos derivados de la globalización que permea todos los sectores sociales. Para los jóvenes, la escuela se presenta como un espacio en el que se pueden desarrollar mejor estas habilidades, ya que su alumnado está en plena formación cognitiva, actitudinal y socioemocional. Esta investigación se justifica por la necesidad de aclarar cómo la escuela, especialmente el profesor, puede contribuir a la formación autónoma y dinámica de alumnos y alumnas que estudian en escuelas públicas y se revelan vulnerables a los desafíos sociales. E estudio tiene como objetivo analizar la interacción social entre profesores y alumnado, centrándose en la aplicación de técnicas pedagógicas capaces de desarrollar la resiliencia, en una escuela secundaria de una comunidad vulnerable, a partir de la triangulación de los marcos teóricos de la interacción, la exclusión y la autonomía. La metodología se basa en un enfoque cualitativo de carácter exploratorio con el método de estudio de caso. Los datos se recogen mediante el análisis de documentos, la observación y las entrevistas con ocho profesores/profesoras y veinte alumnos/alumnas de una escuela pública de la capital de Brasil. Como resultados más relevantes de la investigación, se observa que la interacción profesor-alumno dentro del proceso de enseñanza-aprendizaje hace que el proceso educativo sea significativo para el alumnado y la estrategia pedagógica posibilita la formación integral de alumnos/ alumna ante los retos que surgen de la realidad actual.

Descriptores: Resiliencia, inclusión, interacción, vulnerabilidad, estrategias pedagógicas, la escuela secundaria.

\section{Abstract}

Society demands autonomy, dynamism, and protagonist skills in the face of the most diverse challenges arising from globalization that permeates all social sectors. For young people, the school presents itself as a space in which these skills can be better developed since their students are in full cognitive, attitudinal, and socio-emotional training. This research is justified by the need to clarify how the school, especially the teacher, can contribute to the autonomous and dynamic formation of students who study in public schools and reveal themselves to be vulnerable to social challenges. The study aims to analyze the social interaction between teachers and students, focusing on applying pedagogical techniques capable of developing resilience, in a secondary school in a vulnerable community, based on the triangulation of theoretical frameworks of interaction, exclusion, and autonomy. The methodology is based on a qualitative approach of an exploratory nature with the case study method. Data are collected through document analysis, observation, and interviews with eight teachers and twenty students from a public school in the capital of Brazil. As the most relevant research results, it is observed that the teacher-student interaction within the teachinglearning process makes the educational process meaningful for students and pedagogical strategy enables the comprehensive training of students in the face of the challenges that emerge from reality current.

Keywords: Resilience, inclusion, interaction, vulnerability, pedagogical strategies, high school.

Forma sugerida de citar: Rezende, R., \& Caliman, G. (2022). Interacción, diálogo y prácticas pedagógicas en el bachillerato. Alteridad, (17)1, 103-113. https://doi.org/10.17163/alt.v17n1.2022.08 


\section{Introducción}

La sociedad, desde su constitución, hace distinciones entre las personas, clasificándolas por sus características físicas, psicológicas y por su posición social. Actualmente, el discurso del respeto a las diferencias se intensifica, siendo el foco la búsqueda de la igualdad, y según la Declaración Universal de los Derechos Humanos, "todos los seres humanos nacen libres e iguales en dignidad y derechos" (ONU, 2018). A su vez, la Constitución Federal brasileña establece en su artículo $5^{\circ}$ que "todos son iguales ante la ley, sin distinción alguna” (Brasil, 1988). Sin embargo, entre lo que se dice y lo que se hace, hay una brecha, y en el caso brasileño, la falta de respeto a las diversas formas de diferencias sociales ha asumido un carácter histórico, llegando a los ambientes escolares.

Considerando el derecho a la igualdad y a la dignidad para la educación, que es el foco de esta investigación, es posible identificar al alumno de una comunidad vulnerable que vive bajo adversidades, rodeado de problemas familiares, violencia, invisibilidad social/educativa, y con dificultades en la interacción con sus profesores. Se enfrenta a las fuerzas de un círculo de exclusión que puede influir en toda su vida social y educativa.

A pesar de este contexto desafiante, algunos alumnos logran salir de esta situación y se liberan de estos ciclos. Los que no logran salir sufren directamente los efectos de una red de factores discriminatorios ubicados fuera de la escuela (violencia, drogas, discriminación y problemas sociales), que terminan retroalimentando la exclusión educativa a través de la indisciplina, las bajas calificaciones, el fracaso y la deserción escolar; todo esto se traduce en un previsible fracaso escolar.

Para cambiar este escenario, la interacción surge como una posible diferencia entre el estancamiento y la superación, la exclusión y la interacción, y el diálogo para construir la resiliencia necesaria frente a los obstáculos que ya enfrentan estos alumnos.
$\mathrm{Al}$ analizar el concepto de interacción, en lo que respecta a la educación, se puede definir como la relación de influencia recíproca en la que el comportamiento de los involucrados se convierte en un estímulo para el otro (Síveres, 2016; Caliman, 2008). La interacción profesoralumno se produce entre dos actores fundamentales para la acción educativa: profesor y alumno. Esta interacción presenta a estos actores como complementarios y estructurados entre sí. Uno condiciona la existencia del otro y las acciones de ambos se orientan mutuamente.

En resumen, la resiliencia puede entenderse como la capacidad de resistir una condición desfavorable. Existe la posibilidad de desarrollar la resiliencia en diferentes grados a lo largo de la vida del individuo, y puede ser desarrollada/estimulada en cualquier grupo social o institución (Assis et al., 2006).

Analizando la educación brasileña, se puede observar cómo está en desventaja en comparación con otros países: aumentan los problemas como la deserción escolar, la brecha de conocimientos y los problemas sociales que afectan el desempeño del alumnado en la escuela. Estos problemas ponen en evidencia lo que se puede hacer para revertir esta situación, ya que el mundo está cada vez más globalizado, con nuevas tecnologías y exige nuevas competencias. En este mundo que se desarrolla rápidamente, se requiere que los individuos desarrollen sus potencialidades (Luckesi, 2016) para convivir con estos nuevos desafíos y superarlos. La resiliencia (Laplane, 2000), como ya se ha mencionado, es un factor que contribuye al cambio de paradigmas negativos. La resiliencia, desarrollada y mejorada a través de la educación, puede ser una herramienta esencial para cambiar el marco en el que se presenta la educación brasileña. Para ello, la educación se erige como el lugar adecuado para el desarrollo de las potencialidades.

Así, este estudio busca presentar cómo la interacción entre profesor y alumno en una escuela pública de una comunidad vulnerable 
puede cambiar este cuadro de fracaso a través del desarrollo de la resiliencia en el ambiente escolar.

\section{Metodología}

La investigación es cualitativa y exploratoria, utilizando el método de estudio de caso (Stake, 2016). La población investigada fue la de una escuela pública de Ceilândia, en el Distrito Federal. La escuela resiente una importante vulnerabilidad relativa a cuestiones socioeconómicas, violencia, problemas familiares, embarazo adolescente, drogadicción y pobreza. Los alumnos y las alumnas estaban matriculados en la escuela secundaria de turno diurno. Fueron entrevistados ocho profesores y veinte estudiantes; se observaron dieciséis clases de Lengua Portuguesa y Matemáticas.

La elección de una escuela pública en una comunidad vulnerable se justifica porque el artículo 206, inciso VII, de la Constitución Federal (Brasil, 1988) establece la garantía de estándares de calidad para la educación. Sin embargo, existen diferencias entre la garantía de este derecho en relación con las comunidades vulnerables y las no vulnerables. La escuela secundaria fue el campo de investigación porque es la última etapa de la educación básica, en la que el alumnado debe presentarse como capaz de ejercer su ciudadanía y entrar en el mercado de trabajo o en la Universidad (Brasil, 1988).

En la generación de datos se utilizó el razonamiento deductivo e inductivo, la aproximación a la realidad y el análisis bibliográfico de teóricos que abordan los temas de la interacción profesor-alumno, el diálogo y la resiliencia. La investigación comenzó con un análisis documental, analizando, a priori, el Proyecto Político-Pedagógico (PPP) de la escuela, y los planes de curso de los profesores seleccionados para la investigación. El objetivo de este análisis fue identificar las posibles estrategias pedagógicas durante la interacción profesor-alumno que contribuyeran a la resiliencia y, en consecuencia, a la inclusión de alumnos de esta escuela. Se rea- lizaron entrevistas semiestructuradas y observación participante. $\mathrm{P}$ se destinará a los profesores, seguido del número asignado para identificar a los profesores y a los alumnos citados en el estudio. Lo mismo ocurre con los alumnos. Serán identificados por la letra A seguida de un número correspondiente al orden de colocación de las entrevistas.

\section{Análisis de datos y resultados}

El análisis de los resultados se basó en la interacción profesor-alumno y cómo se presentó en los temas identificados: los círculos de exclusión identificados en la investigación, las estrategias pedagógicas utilizadas por profesores y el diálogo construido a través de la interacción observada entre profesor y alumno.

$\mathrm{Al}$ abordar primero el análisis documental, este se basó en el Proyecto Político Pedagógico de la Escuela Pública Pesquisada-EPP y en los planes de curso de los profesores. La observación se basó en un guion. Sin embargo, la información pertinente, que fue notada a lo largo de la recolección, también fue insertada en la descripción presentada. Las entrevistas fueron semiestructuradas, siguieron los guiones previamente elaborados y abordaron puntos relevantes presentados en las observaciones y en el guion preparado.

\subsection{Círculos de exclusión}

Los alumnos del EPP, en su mayoría, son afrodescendientes, tienen dificultades económicas y problemas familiares importantes. Por lo tanto, se puede percibir varias categorías de excluidos, y pueden experimentar varias situaciones de exclusión (Durkeim, 1999; Weber, 2002). Se observa entre los alumnos de esta escuela cómo sufren debido a la pobreza, la pertenencia racial, la violencia y las condiciones sociales. En los análisis, observaciones y entrevistas, se evidenciaron dos círculos excluyentes, uno formado por alumnos que presentan alguna dificultad de aprendizaje y otro formado por alumnos con discapacidad. 


\subsubsection{Problemas de aprendizaje}

El primer círculo a tratar es el pedagógico. La EPP tiene unos índices de aprobados insatisfactorios en los cursos de $1^{\circ}$ y $3^{\circ}$ año. Las observaciones en el aula y las entrevistas con los alumnos han permitido constatar un cierto grado de desmotivación hacia el estudio, especialmente entre los alumnos de $3^{\circ}$ año. Esta situación puede ser ejemplificada por la alumna A1, que está en el tercer año y no ha decidido qué carrera quiere seguir, no se ha inscrito en el Enem, es decir, no tiene ningún enfoque sobre su futuro, y afirma que "... solo quiero terminar mis estudios, eso es todo".

En el curso de 1er año, lo que más destacó fue la queja recurrente sobre la diferencia entre el $9^{\circ}$ curso de Primaria II y el ler año de Secundaria. También se criticó que no se trabaje la transición de Primaria a Secundaria con los alumnos de los últimos cursos de Primaria II, ni con los alumnos recién llegados a 1er año.

En el Proyecto Pedagógico de la EPP, hay varias referencias a la preocupación con la atención de los alumnos con bajo rendimiento, con la falta de conocimientos previos y necesarios para algunos contenidos, y con los alumnos desinteresados y ausentes. La EPP orienta a los profesores sobre los alumnos ausentes o que tienen dificultades de aprendizaje para que se pueda hacer un trabajo diferenciado con ellos. Esta actitud inclusiva de la EPP permite acoger a estos alumnos excluidos en las aulas y que necesitan encontrar viabilidad para ser incluidos y visibles en la escuela y posteriormente en la sociedad.

Como propuesta para afrontar las dificultades/desinterés, la EPP sugiere proyectos pedagógicos y otras actividades que pretenden contribuir al desarrollo total del alumno y romper este círculo negativo. En la APP se mencionan proyectos interdisciplinarios. Algunas actividades fomentan la participación en pruebas, como el PAS, el Enem, los concursos, las pruebas de acceso, que se aplican al final del bachillerato, y otras actividades como la Olimpiada de Matemáticas y Astronomía.
En la parte diversificada, que son las clases extras distribuidas para completar la carga mínima exigida a cada profesor, la EPP propone actividades para superar algunas de las dificultades de los alumnos ya identificadas en las evaluaciones y/o en las observaciones de aula. En estas clases se sugieren algunos proyectos que implican talleres de lectura y escritura, el rescate de las matemáticas para los alumnos con grandes dificultades, actividades en la sala de informática y el fomento de las clases de lengua extranjera (español) y matemáticas.

En las observaciones realizadas, el trabajo de asistencia a los alumnos con dificultades de aprendizaje no fue tan tangible como se presenta en el PPP. Las clases de seguimiento no se realizaron este año, como se esperaba, debido a la falta de alumnos. Eso ocurrió porque la mayoría de los profesores trabajan en horario extraescolar. Las prácticas destinadas a mejorar la lectura y la interpretación, dificultades mencionadas en el PPP, se observaron en las clases de los profesores P2 y P5, que trabajan con proyectos transdisciplinarios basados en temas de la vida cotidiana de los alumnos.

En cuanto a los problemas de aprendizaje en el área de matemáticas, se observaron dos acciones más significativas: el seguimiento realizado por el profesor de Biblioteca que ayuda a los alumnos que lo solicitan; y las intervenciones que el profesor $\mathrm{P} 1$ realiza dentro del aula centradas en actividades de prerrequisito, es decir, contenidos previos y necesarios centrados en conceptos matemáticos con el objetivo de contribuir a la recuperación progresiva de conocimientos en esta materia.

En las entrevistas con los profesores, la percepción sobre la atención a las dificultades de los alumnos fue bastante diferente. Cuatro de los entrevistados señalan la falta de interés de la mayoría de los alumnos que desanima el trabajo pedagógico. Sienten la necesidad y la obligación de contribuir a reducir las dificultades percibidas en las clases y en las evaluaciones. Para los profesores, los alumnos muestran poco 
interés en reparar los prerrequisitos o en trabajar las dificultades de lectura o interpretación, por ejemplo. Según los cuatro profesores, la mayor preocupación percibida entre los alumnos era la de obtener la nota mínima requerida para aprobar. Esta actitud fue más recurrente en el ler año. En el 3er año, se pudo verificar una preocupación más significativa con los estudios a causa del Enem y de los exámenes de acceso a la universidad. También se observa que algunos alumnos se interesan exclusivamente por aprobar el examen con una nota mínima.

Los alumnos, en sus entrevistas, afirmaron que los participantes de los proyectos de incentivo a la lectura y a la interpretación tuvieron un mejor desempeño durante el semestre que los alumnos que no participaron y que se interesaron más en las clases y participaron efectivamente en las actividades propuestas.

\subsubsection{Alumnos especiales}

En el PPP de la escuela, la inclusión de alumnos con discapacidad está contemplada en la meta 4 del Plan Nacional de Educación-PNE (Brasil, 2014). Para los alumnos con discapacidad, la EPP ofrece Asistencia Educativa Especializada-AEE. ${ }^{1}$ El Aula de Recursos ${ }^{2}$ es responsable de garantizar la participación de los alumnos en el aula regular, orientando y controlando la aplicación de pequeñas o grandes adaptaciones curriculares (Góes \& Laplane, 2004), siendo implementadas por los profesores para proporcionar un mejor aprendizaje a estos alumnos.

En el aula regular, se notó una interacción más significativa entre los profesores y estos alumnos. También había actividades que intentaban mostrar, de diferentes maneras, el contenido para que tuvieran mejores condiciones de aprendizaje. La interacción de los profesores con sus alumnos es más celosa, y se anima a estos alumnos a participar en clase y en la resolución de ejercicios. Los alumnos también participan en actividades grupales mixtas dentro del aula donde son evaluados.
En las entrevistas con los dos profesores que trabajan con alumnos con discapacidad, destacaron la preocupación por atender mejor las necesidades específicas de este colectivo. Los profesores creen que aún queda mucho por hacer para desarrollar e incluir a estos alumnos. Confirman que hubo una mejora entre algunos alumnos, que ahora pueden participar más eficazmente en las actividades pedagógicas, presentar trabajos en grupo, hacer exámenes en salas normalizadas y socializar durante los recreos.

En la entrevista con los dos alumnos con discapacidad, destaca el entusiasmo que expresan por estar estudiando. Pasan más tiempo en la EPP que el resto de los alumnos porque necesitan asistir al Aula de Recursos algunos días por la mañana, además de todas las tardes en el aula ordinaria.

Así, la EPP atiende tanto a alumnos con problemas de aprendizaje como a alumnos excepcionales, representando los dos círculos de exclusión identificados en la investigación. Sin embargo, a través de las entrevistas y las observaciones, se pudo ver que el círculo de alumnos especiales tiene más interacción por parte de toda la EPP. Posiblemente esto ocurre porque tienen a su favor la legislación que ordena actividades que atiendan sus necesidades.

\subsection{Estrategias pedagógicas}

"Las estrategias pedagógicas" se entienden aquí como los procedimientos planificados e implementados por los educadores para contribuir al aprendizaje de sus alumnos. Deben ser diversificadas y creativas para atender a los diferentes contenidos y necesidades de los alumnos que asisten al aula (Antunes, 2015). En una visión general, las prácticas pedagógicas que más contribuyen a la formación de alumnos tienen afinidad con el concepto de metodologías activas (Revans, 2011). Conciben a los alumnos como agentes y protagonistas de su aprendizaje, y en algunos de los planes presentados se utilizaron metodologías activas. 
Así, tanto en el PPP como en las orientaciones para la elaboración del plan de curso, se evidencia que la escuela considera que la forma de enseñar tiene que ser innovadora, eficaz y diversa. Deja claro que se necesitan estrategias que contribuyan a la formación cognitiva y actitudinal del alumno. Al analizar, en general, los planes de curso presentados por los profesores de la EPP, es fundamental observar que, a pesar de las muchas estrategias pedagógicas que pueden ayudarlos en el aula, las más mencionadas en los planes fueron las tradicionales (lectura, debate y conferencia).

El primer plan de curso analizado fue el de Matemáticas. Presenta como estrategias la clase expositiva y participativa, la formación de equipos para resolver ejercicios, actividades de investigación extra-clase, la observación y la participación de alumnos en aspectos actitudinales. La clase expositiva y participativa es una primera estrategia que puede contribuir al desarrollo de la resiliencia porque, además de la interpretación y discusión de los contenidos presentados en clase, el alumno puede desarrollar la capacidad de diálogo y empatía. Esta estrategia permite al alumno desarrollar las habilidades de visión dialogante, protagonismo, reflexión y evaluación del contexto.

La segunda estrategia tiene como objetivo la resolución de problemas a través de juegos operativos. Están orientados a promover la reflexión, la búsqueda de soluciones, el diálogo, la participación colaborativa y el análisis de posibilidades (Koehler et al., 2010). El profesor P1 impartió la clase de forma expositiva, con interacción y fomento de la participación de alumnos en general. Este profesor presenta un buen diálogo con la clase, anima al grupo con bromas y frases de incentivo, y estimula la participación para que hagan sus preguntas. Para explicar el contenido de la clase, realiza actividades con ejemplos significativos basados en la realidad de la comunidad y con elementos concretos. El profesor destaca que estas actividades son necesarias para facilitar la comprensión y el aprendizaje; todos los ejercicios se resuelven con la partici- pación y la aportación de los alumnos. Todos ellos permanecen cerca de la silla del profesor y prestan atención a todo lo que enseña.

En el plan de Lengua Portuguesa de 3er año se incluyen estrategias de estudio dirigido e investigación en los cuatro bimestres. Ambas prácticas pedagógicas ayudan al desarrollo reflexivo, crítico y autónomo de alumnos. Además de estas estrategias, los talleres literarios promueven el desarrollo de la creatividad. La planificación de $3^{\circ}$ año también incluye una práctica llamada retroalimentación que contribuye a desarrollar el pensamiento crítico, la aceptación y el respeto. La reescritura de ensayos/ textos ayuda al desarrollo de estas competencias. La planificación del $1^{\circ}$ año de Lengua Portuguesa incluye actividades como las salidas de campo, que favorecen el desarrollo del aprendizaje en grupo, la observación, la concentración y el juego y desarrollan la creatividad, la reflexión y la lectura del mundo (Koehler et al., 2010).

La observación del $1^{\circ}$ año en el aula resulta de una divergencia entre lo planificado y la práctica. Los profesores P3 y P4 no utilizaron las estrategias pedagógicas diversificadas contempladas en la planificación. Las clases fueron solo expositivas y sin incentivos para la participación de los alumnos, lo que contribuyó a su dispersión.

Algunos profesores trabajan las dificultades de lectura e interpretación a través de proyectos transdisciplinarios. En el primer semestre, el proyecto destacado fue realizado por P2 y P5, profesoras de $3^{\circ}$ año. Las actividades se basaron en la Ley Maria da Penha (Brasil, 2006) y se realizaron a través de debates, producción de textos, entrevistas, seminarios, cordéis, ensayos y preparación de vídeos. Todas ellas son estrategias pedagógicas, como afirmó Antunes (2013), que ayudan significativamente al aprendizaje del alumno. La participación de los alumnos fue visible, y las producciones fueron trabajadas en el aula, en casa y como actividades evaluativas. Los videos realizados por alumnos fueron inscritos en la "Mostra de Curta de Brasília", 2019, resultando en la obtención del primer lugar, comprobando su crecimiento en el 
ámbito pedagógico y en la promoción de la ciudadanía. Esa satisfacción puede ser ratificada en el discurso de alumnos A2, que expresa su orgullo por el "[...] resultado de nuestro trabajo" y A6 que reconoce lo mucho que "fue genial trabajar en clase sobre temas comunes para nuestra comunidad y nosotros".

Para trabajar las competencias sociales y emocionales, observamos el proyecto de profesores $\mathrm{P} 2$ y P5, extendido a toda la escuela, que aborda la formación multidimensional de alumnos. El proyecto tiene como objetivo la formación ciudadana y el desarrollo de la empatía en el alumnado. El trabajo cosecha resultados con el cambio de comportamiento actitudinal, conciencia ciudadana y participación en la realidad del alumno dentro y fuera de la escuela, ya que trabaja el respeto, la empatía y la vida en sociedad.

Otro proyecto excelente para la formación integral del alumno, también de profesores P2 y P5, se centró en la valorización de la mujer dentro de la propia familia. Esta valorización se presenta como relevante porque el PPP de la escuela registra que las mujeres (abuelas y madres) sostienen la mayor parte de las familias de la comunidad. El trabajo generó respeto, admiración y un cambio en el comportamiento de alumnos hacia la vida de esas mujeres. Además de las prácticas dirigidas al diálogo, a través del desarrollo de la producción de textos, debates y exposiciones en la escuela, se observa que hubo un desarrollo en las actitudes de los alumnos hacia la alteridad en la familia. Los alumnos destacan el placer de tener clases que tengan que ver con su realidad (Wallon, 1968), posibilitadas a través de actividades prácticas y no solo de contenidos estancos.

En cuanto a los profesores, todos están de acuerdo en contribuir con las estrategias pedagógicas a la formación integral del alumno. Sin embargo, cuatro profesores creen que el alumnado no se compromete como debería con las actividades propuestas. ¿La falta de compromiso no sería una falta de relación entre las actividades propuestas y la realidad vivida por los alumnos?

\subsection{El diálogo}

El diálogo es la base para la comunicación, y la necesidad de una relación dialógica entre alumno y profesor es incuestionable para la interacción. Por ello, el profesor puede influir positivamente a través del diálogo polisémico y ontológico porque su proximidad a los alumnos le permite conocer e interactuar eficazmente con el proceso pedagógico.

Así, el diálogo se revela como un proceso relacional que influye en las relaciones personales, epistémicas y ontológicas. El ser humano es un individuo convivencial que dialoga consigo mismo, con el otro, con la cultura y con la naturaleza. Por eso, en la escuela, el diálogo debe ser intencional porque, a través de él, existe la posibilidad de realización personal y de transformación social, según la pedagogía de Freire.

A partir de las palabras de Freire (1992) y de Síveres (2016), es posible verificar la eficacia del diálogo entre profesores y alumnos, y esto fue atestiguado en dos momentos diferentes en la EPP. En la clase del profesor P1, la conversación es espontánea y agradable, está atento a los comentarios en clase y motiva a sus alumnos a participar en todas las actividades. En las clases de la profesora P2, la conversación se inicia en los pasillos de la escuela hasta llegar al contenido propuesto para el día. Cada vez que aparece un vínculo con la vida o la realidad de los alumnos (la violencia en el barrio, las políticas públicas y el contexto social), este vínculo se inserta inmediatamente en el contexto del aula para que todos puedan también discutirlo.

En las observaciones del aula, notamos la diferencia entre las clases con prácticas pedagógicas motivadoras, inspiradoras y dialogantes y las clases de "tiza y pizarra" con solo "monólogo del profesor".

Las observaciones realizadas revelan que las prácticas puestas en marcha en algunas aulas están en contradicción con la realidad. Mientras que en algunas clases el diálogo es natural y espontáneo; en otras, reina el monólogo del pro- 
fesor. Aunque la conversación es aparentemente constante en el aula, el diálogo se limita a alumnos y a los grupos que se forman dentro del aula. La mayoría de profesores parece que solo establecen prácticas dialógicas cuando exigen silencio y atención o recuerdan a los alumnos que una actividad concreta merece una calificación.

Las clases sin interacción y diálogo resultan en la desvinculación de los alumnos, generando dispersión y apatía (Moreira, 2010). Los alumnos estaban presentes en el aula, pero sin participación efectiva, y cuando sonaba el timbre para terminar la clase, la estampida era incontrolable. El profesor descargaba su contenido y se marchaba para repetir este comportamiento en la siguiente clase.

Tres de los ocho profesores entrevistados empezaron a tener a los alumnos en los pasillos de la EPP y a los que ya habían entrado en el aula, manteniendo una conversación receptiva sobre cómo van los alumnos hasta llegar a lo que pedagógicamente va a funcionar. Las clases con estrategias pedagógicas se llenaron de diálogo, discusiones, debates y empatía. La participación de los alumnos resultó significativa, de modo que durante la clase se ejerció el respeto, la conversación y la exposición de opiniones similares u opuestas.

Quince de los veinte alumnos entrevistados también destacaron que la forma en que el profesor habla e interactúa con ellos marca mucha diferencia; esta relación, positiva o negativa, se traduce en el nivel de participación de alumnos en las actividades y en lo motivados que se sienten o no para exponer sus ideas durante la clase o en las actividades propuestas (Assis et al., 2008).

Esta diferencia en el uso del diálogo por parte de profesores se señala en las entrevistas con los alumnos y se ejemplifica en las declaraciones de los alumnos: A7 - "Hay profesores que no hablan con nosotros. Algunos profesores no nos tratan con educación" - y A2 - "Hay profesores que son nuestros amigos".

\section{Discusión y conclusión}

La interacción es la relación recíproca de estímulo e influencia entre las personas. La interacción está presente en la relación profesor-alumno en la escuela y es una herramienta indispensable para que se construya todo el proceso pedagógico. En la investigación, la interacción se clasificó en función de las estrategias pedagógicas y el diálogo, y estos dos aspectos fueron recurrentes o estuvieron ausentes. Estas dos categorías son esenciales para que la interacción positiva ayude al aprendizaje y al desarrollo pleno de los alumnos; y para que el diálogo, herramienta indispensable para el proceso pedagógico y social, ocurra en el aula. Así, la ausencia de estrategias y de diálogo influyen negativamente en todo el proceso educativo; y lo contrario también es cierto, ya que las estrategias y el diálogo influyen positivamente en dicho proceso.

Es necesario construir y privilegiar el proceso interactivo entre profesor-alumno en la escuela. Como señala Wallon (1968), la escuela es un recurso significativo para el desarrollo, ya que, desde los primeros años de la vida escolar, se convierte en el centro de las relaciones que guían el desarrollo cognitivo, interactivo y afectivo del alumno. De esa forma, los ambientes constituyen al individuo, y la escuela es uno de esos ambientes.

Según Tavares (2002), la resiliencia está vinculada a las habilidades individuales y puede construirse mediante la interacción con las personas y su contexto. Así, en lo que respecta a los profesores y a las observaciones realizadas, es posible afirmar que el desarrollo de la resiliencia en algunos alumnos se logró a través de la actitud de algunos profesores que se preocuparon por desarrollar estrategias para favorecer la resolución de ejercicios prácticos, prácticas pedagógicas y asesorías dirigidas a la continuidad de los estudios. Este comportamiento refuerza el papel de la escuela y de profesores porque ambos son inductores de la resiliencia y pueden influir positivamente de manera individual en la formación 
de la autonomía, el autocontrol y el carácter de los alumnos (Caliman, 2012).

Los profesores también consideraron positiva la interacción entre profesor y alumno. Sin embargo, algunos profesores se mantienen distantes de los alumnos, entrando en el aula solo para pasar los contenidos programados y presentar su monólogo, haciendo poco para que las relaciones en el aula sean más significativas para ambos.

Una escuela que promueve el diálogo (Buber, 2003), que acoge y despierta la confianza y la autonomía, contribuye a la construcción o al desarrollo de la resiliencia en los alumnos (Assis et al., 2006). Al construir o desarrollar la resiliencia, se rompe el vínculo con una cultura pesimista que tiende a consolidarse dentro del proceso educativo. Esta cultura se basa en la falta de interés de los alumnos, la falta de compromiso de los profesores, la drogadicción cada vez más presente en las escuelas y la violencia social y doméstica, lo que lleva a los alumnos de comunidades vulnerables al fracaso recurrente e inevitable escolar y de vida.

Todos los alumnos y alumnas necesitan oportunidades que promuevan un aprendizaje significativo y diverso, y los que viven en comunidades vulnerables necesitan aún más estímulo para aprender, de modo que puedan romper con los procesos de exclusión que los acosan y prefiguran nuevas perspectivas de vida. Teixeira (2016) presenta cuatro pilares de resiliencia, humor, creatividad, optimismo y motivación. Estos pilares se pueden observar en los proyectos realizados por la EPP para promover el desarrollo de la creatividad y el optimismo y motivar a los alumnos a cambiar frente a la realidad que viven.

En las observaciones se comprueba cómo el alumnado no hace lo propuesto porque no sabe cómo hacerlo, y esto se explica también por el desfase formativo (entre la edad del alumno y la clase a la que asiste) que se acumula, repercutiendo en la formación académica de los alumnos desde la Escuela Primaria. Cuando el alumno llega a la Secundaria, la idea es que todo depende de él, y termina siendo responsable de la construcción de su propio conocimiento. Muchas veces, la actitud del alumno con un desfase formativo llega a ser vista como desinterés y apatía, resultando, no pocas veces, en la deserción escolar y la repetición recurrente.

Si la formación del alumno de bachillerato estuviera basada en lo que afirman Marandino y Scarpa (2017), esta etapa educativa debería ser el espacio para que la información y el conocimiento se basen en la reflexión, teniendo la formación del alumno en su totalidad. Para que esto ocurra, el alumno debe ser parte de todo el proceso pedagógico, contribuyendo con su perspectiva ontológica y epistemológica, desarrollando el poder de leer el mundo y, en consecuencia, desarrollando su resiliencia a los desafíos de la escuela y de la sociedad. Todo debe hacerse en colaboración entre la escuela y el alumno, como ya lo planteaba Don Bosco (Hornich \& Biscalchin, 2018) en sus trabajos con los y las jóvenes.

Desarrollar una visión de formación integral es esencial y se reafirma a través de que la escuela logre construir una cultura resiliente, donde el desarrollo de competencias favorezca la inclusión dentro y fuera de la escuela. Una cultura formada a partir de la actuación de la institución educativa, a través de sus educadores, dirigida a influir en la historia de vida de cada alumno. De esta manera, sí es posible eliminar el estigma negativo de que la escuela promueve la exclusión, como se ha venido presentando.

En esta perspectiva, la formación del profesor debe basarse en otras áreas además de la cognitiva. Debe presentar estrategias pedagógicas positivas y promover la interacción en el aula. Así, la escuela puede convertirse en un ambiente propicio para la formación integral de los alumnos de secundaria, creando posibilidades para el cambio de la cultura del fracaso y de la falla a una cultura de la inclusión de la resiliencia de la superación.

El camino para hacer del Bachillerato un periodo de aprendizaje concreto y de formación integral del alumno debe ser gradual pero 
constante. Este cambio requiere ciertamente de políticas educativas que valoren la educación en general y que requieran la participación del profesor. El profesor debe estar dispuesto a cambiar el paradigma educativo que se ha creado y consolidado en los últimos siglos. Hay que animar al profesor a desarrollar una conciencia de su responsabilidad en la promoción del aprendizaje del alumno. Para ello, es necesario proporcionarle oportunidades de formación continua (Imbernón, 2010). El profesor debe tener acceso a formación para utilizar nuevas estrategias pedagógicas, como las metodologías activas (Revans, 2011), para ser valorado como ciudadano y profesional, consciente de su papel determinante en el rendimiento de los alumnos.

\section{Notas}

1 Asistencia obligatoria garantizada por el Decreto No 6.571/08 y la Ordenanza No 243, del 15 de abril de 2016.

2 Espacio físico garantizado por la ley (Resolución SE 68, de 12-122017).

\section{Referencias bibliográficas}

Antunes, C. (2013). Na sala de aula (In the classroom) (3a ed.). Editora Vozes.

https://amzn.to/3aNOD9h

Antunes, C. (2015). Resiliência-a construção de uma nova pedagogia para uma escola pública de qualidade (fascículo 13) (8a ed.). Vozes. https://amzn.to/3AVydpQ

Assis, S. G., Avanci, J. Q., Pesce, R. P., \& Njaine, K. (2008). Resiliência na adolescência: refletindo com educadores sobre a superação de dificuldades. [Resilience in adolescence: reflecting with educators on overcoming difficulties] Fiocruz/ENSP/Claves/CNPq.

https://bit.ly/3ARG1sO

Assis, S. G., Pesce, R. P., \& Avanci, J. Q. (2006). Resiliência: enfatizando a proteção dos adolescentes. Artmed. https://bit.ly/3ASxFB5

Bauman, Z. (2001). Modernidade líquida. Tradução de Plínio Dentzien. Jorge Zahar.

https://bit.ly/3AMisBL
Brasil (1988). Constituição da República Federativa do Brasil. Diário Oficial da União - Seção 1 - 5/10/1988, Página 1 (Publicação Original). D.O.U de $05 / 10 / 1988$, pág. ${ }^{\circ} 1$

Brasil (2006). Lei no 11.340, de 7 de agosto de 2006. Câmara dos Deputados.

https://bit.ly/3n7DOVa

Buber, M. (2006). (Eu e Tu. Tradução, Introdução e Notas de Newton Aquiles Von Zuben. Centauro Editora. 6. ed.

https://bit.ly/3BUdwMv

Durkheim, É. (1999). Da divisão do trabalho social. $2^{\circ}$ ed. Martins Fontes. https://bit.ly/3vlLRS5.

Caliman, G. (2008). Paradigmas da exclusão social. Unesco. https://bit.ly/3C1I3rA

Caliman, G. (2012). Educação social entre redes afetivas e institucionais. In C. A. Gomes, A. F. Grasiele \& S. M. F. Koehler (Orgs.). Culturas de violência, culturas de paz; da reflexão à ação de educadores, operadores do direito $e$ defensores dos direitos humanos.

https://amzn.to/3jcyPS4

Freire, P. (1992). Pedagogia da Esperança (4a ed.). Paz e Terra. https://bit.ly/3nannHY.

Góes, M. C. R., \& Laplane, A. L. F. da. (2004). Políticas e práticas de educação inclusiva. Autores Associados. https://amzn.to/30DLSWj.

Hornich, D., \& Biscalchin. F. C. (2018). 200 anos de Dom Bosco: a Pedagogia Salesiana, a Universidade para a Maioridade e a Primazia da Dignidade da Pessoa Humana [200 years of Don Bosco: the Salesian Pedagogy, the university for the majority and the primacy of the dignity of the human person]. SérieEstudos, (23), 279-304.

https://doi.org/10.20435/serie-estudos.v23i48.1118

Imbernón, F. (2010). Formação continuada de professores. Artmed. https://amzn.to/3aNymkB.

Koehler, S. M. F., Santos, G. J. P. A., Correa, C. F. S. (2021). Adolescentes en la escuela: grupos de reflexión para pensar en la práctica de la ciudadanía. [Adolescents in school: reflection groups to think about the practice of citizenship] Alteridad Revista, 16(2), 249-260, https://doi.org/10.17163/alt.v16n2.2021.07

Laplane, A. L. F. (2000). Interação e silêncio na sala de aula. Editora. Unijuí. https://bit.ly/3aN83Ll 
Luckesi, C. (2016, 21, agosto). O ser humano, sua educabilidade e o educador. Luckesi.blogspot. https://bit.ly/3aOplrA

Marandino, M., \& Scarpa, D. L. (2017). Diálogos entre a formação integral e a alfabetização científica no Ensino médio. In R. Gauche \& W. Weller, Ensino médio em debate: currículo, avaliação e formação integral. 1ª. Ed. (pp. 167200) [High School in Debate: Curriculum, Assessment and Comprehensive Education]. Editora UnB. https://bit.ly/31QB2nV.

Moreira, I. (2010). Fracasso escolar e interação professor-aluno. (3a ed.). Editora Wak.

https://amzn.to/2XsMDAk

ONU-Organização das Nações Unidas (2018). Declaração Universal dos Direitos Humanos. https://bit.ly/3n67CkZ
Revans, R. (2011). ABC da aprendizagem pela ação. Farnham: Gower.

http://doi.org/ 10.37702/COBENGE.2020.2792

Síveres, L. (Org.). (2016). Diálogo-um princípio pedagógico. Liber Livro. https://bit.ly/3jdhjNn

Stake, R. E. (2016). A arte da investigação com estudos de caso. Fundação Calouste.

https://bit.ly/3jaLepq

Tavares, J. (Org.). (2002). Resiliência e Educação (3a ed.). Cortez. https://bit.ly/3BUQWDd

Teixeira, E. C. (2016). Resiliência e vulnerabilidade social: uma perspectiva para a educação sociocomunitária da adolescência. Vozes.

https://amzn.to/3DXmXvj

Weber, M. (2002). Conceitos básicos de Sociologia. 1982. https://bit.ly/3DQlgj1

Wallon, H. (1968). A evolução psicológica da criança. Edições 70. https://bit.ly/3BRFb0A 


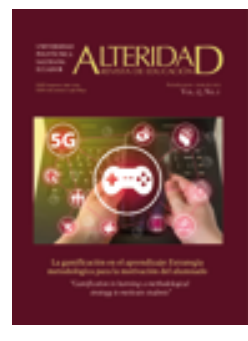

\title{
Estudio motivacional sobre el aprendizaje de la religión en Educación Básica mediante las TIC
}

\author{
Motivational study on learning religion in Basic Education \\ using ICT
}

D Justo Olivares-Rosado trabaja en la Arquidiócesis de Quito (Ecuador) (justo@arquidiocesisdequito.com.ec) (https://orcid.org/0000-0003-1347-9025)

D Dra. Isabel López-Cobo es profesora de la Universidad de Sevilla (España) (ilopez10@us.es) (https://orcid.org/0000-0003-3579-0877)

(D) Dr. Jesús Conde-Jiménez es profesor ayudante en la Universidad de Sevilla (España) (jconde6@us.es) (https://orcid.org/0000-0002-4471-5089)

Recibido: 2021-08-14 / Revisado: 2021-12-02 / Aceptado: 2021-12-05 / Publicado: 2022-01-01

\section{Resumen}

La motivación es un aspecto que sirve de motor para despertar el interés en asimilar una unidad curricular en particular. Esta investigación tiene como objetivo determinar las causas que provocan desmotivación en el estudiantado de Educación General Básica (EGB) de una institución educativa religiosa modelo y su influencia en el interés por aprender sobre religión, con el fin de incorporar las TIC como estrategia motivacional de enseñanza. Metodológicamente, se trata de una investigación bajo el paradigma positivista de carácter no experimental, ex post facto y transversal, de naturaleza descriptiva y causal, con un diseño documental y de campo. El tipo y nivel de profundidad del estudio es descriptivo y explicativocorrelacional con la participación de una muestra de 268 estudiantes y ocho docentes de religión. Se diseñaron dos tipos de cuestionarios, uno dirigido al estudiantado y otro a los/as docentes. La hipótesis consistió en verificar si a mayor motivación y satisfacción del estudiantado, mayor aprendizaje de la religión en Educación Básica. Los resultados evidencian la presencia de un bajo nivel de motivación y satisfacción en el estudiantado a causa de deficiencias en el ambiente de aprendizaje, así como al uso de metodologías de enseñanza tradicionales que limitan la participación activa del estudiantado. Se resalta la necesidad de aplicar metodologías alternativas que incorpore las TIC para incrementar la motivación estudiantil, y, por ende, el rendimiento académico.

Descriptores: Religión, educación, tecnología, información, comunicación, motivación.

\section{Abstract}

Motivation is an aspect that serves as a motor to awaken interest in assimilating a particular curricular unit. This research aims to determine the causes that cause demotivation in the students of Basic General Education (EGB) of a model religious educational institution and its influence on the interest in learning about religion, in order to incorporate ICT as a motivational teaching strategy. Methodologically, it is an investigation under the positivist paradigm of a non-experimental, ex post facto and transversal nature, of a descriptive and causal nature, with a documentary and field design. The type and depth level of the study is descriptive and explanatory-correlational with the participation of a sample of 268 students and eight religion teachers. Two types of questionnaires were designed, one aimed at students and the other at teachers. The hypothesis consisted in verifying if greater motivation and satisfaction of the students, greater learning of religion in Basic Education. The results show the presence of a low level of motivation and satisfaction in the student body due to deficiencies in the learning environment, as well as the use of traditional teaching methodologies that limit the active participation of the student. The need to apply alternative methodologies that incorporate ICT to increase student motivation and, therefore, academic performance is highlighted.

Keywords: Religion, education, technology, information, communication, motivation.

Forma sugerida de citar: Olivares-Rosado, J., López-Cobo, I., \& Conde-Jiménez, J. (2022). Estudio motivacional sobre el aprendizaje de la religión en Educación Básica mediante TIC. Alteridad, (17)1, 114-125. https://doi.org/10.17163/alt. v17n1.2022.09 


\section{Introducción y estado de la cuestión}

Según Arévalo-Tapia (2016), en el Ecuador se evidencia la presencia de una escasa motivación del estudiantado con relación a su deseo por aprender la asignatura de religión, aún más, en las instituciones educativas que siguen un régimen católico cuya materia es parte obligatoria de su malla curricular, aunque para el Estado se convierte en una materia opcional. Al respecto, Núñez-Soler y Lourdes-González (2017) consideran que la falta de motivación y el desinterés propician malos resultados académicos, por lo que es oportuno conocer las causas que lo provocan y su incidencia en el aprendizaje. Holmes-Coto (2018) además resalta que en la actualidad el Ecuador vive un proceso de cambios, actualizaciones curriculares, evaluaciones a las instituciones educativas y capacitación continua a los/as docentes, no obstante, en la parte de la enseñanza-aprendizaje aún se presentan algunas falencias debido al tradicionalismo en la enseñanza donde el estudiantado no tiene un aprendizaje significativo.

En este orden de ideas, el propósito de la investigación consiste en determinar las causas que provocan desmotivación en el estudiantado de Educación General Básica (EGB) de una institución educativa religiosa modelo y su influencia en el interés por aprender sobre religión, a los fines de incorporar las Tecnologías de la Información y la Comunicación (TIC) como estrategia motivacional de enseñanza.

\subsection{La religión en el Ecuador}

A raíz de un estudio desarrollado por el Instituto Nacional de Estadística y Censos en 2012, se expone que en el Ecuador el 91,95 \% de la población afirma profesar alguna religión, de la cual el 80,4\% corresponde a las personas que ejercen el catolicismo (Ecuador en cifras, 2012), por lo tanto, la investigación tiene relevancia al momento de analizar qué es lo que pedagógi- camente impide que el alumnado sienta interés en el aprendizaje de la religión y cómo se podría mejorar la situación.

En este orden de ideas Salinas (2016) señala que las instituciones educativas católicas tienen una alta aceptación por los padres y madres de familias, quienes aspiran para sus representados una formación íntegra que no solo se constituya mediante una educación formal de alto nivel, sino que su educación personal muestre el desarrollo de un conjunto de valores y una conciencia moral bien definida. Actualmente, se observa que estas instituciones no han podido internalizar en el estudiantado la importancia de la religión. Por lo que la investigación arrojará las causas de tal acontecimiento y la posible solución para incrementar la motivación en el estudiantado.

Anticipando a que las causas sean de orden metodológico en la Enseñanza de la Religión Escolar (ERE) y de relaciones entre docentealumno, Salinas (2016, p. 594) manifiesta que "necesitamos estudiar más a fondo el tema de los contenidos y métodos de la Enseñanza Religiosa, dentro de las posibilidades que ofrece el marco escolar, para que pueda desarrollarse como una materia escolar en todas sus consecuencias", es decir, se viene pronosticando la existencia de inconvenientes en el proceso de enseñanza que interfiere en el aprendizaje significativo, $y$, por ende, en la motivación escolar con el propósito de asimilar la educación religiosa a fin de fomentar valores en la personalidad del aprendiz.

\subsection{El aprendizaje como proceso para asimilar significativamen- te la religión escolar}

Existen distintos paradigmas que enfocan el aprendizaje de modo diferente según la premisa que se defienda; al respecto, se señala que a lo largo de la historia y evolución educativa han aparecido y siguen vigentes el paradigma conductista y el constructivista. Estos se describen a continuación: 


\subsubsection{Paradigma conductista}

Su precursor, John Broadus Watson (1878-1958), fue el creador de la escuela conductista, considerando que la psicología debía encargarse del estudio de los procesos observables de la conducta humana, con esta definición sobre el objeto de estudio de la psicología, la colocaba cómodamente dentro de la corriente positivista y excluía de su interés investigativo todo aquello que no fuera observable, medible y verificable (Morales-López \& Irigoyen-Coria, 2016).

Durante su etapa como profesor asistente recibió la influencia de Ivan Pavlov en la cual concuerda que ni a la mente ni a la conciencia pueden recibir un trato netamente objetivo (Garello, 2019). Con respecto a los aportes que brinda el conductismo a la pedagogía, Salas (2002) señala que "el propósito del conductismo es otro que moldear la conducta a través de la manipulación intencionada del ambiente" (p. 13). Asimismo, expresa que, bajo el paradigma conductista, la metodología de enseñanza se enmarca en el establecimiento de objetivos cuyos resultados pueden ser mensurables mostrando un enfoque cuantitativo con desarrollo progresivo en el rendimiento académico del estudiantado. Por otro lado, Sánchez (2012) confirma que 'la pedagogía en la teoría de aprendizaje conductista, se enfoca en una didáctica que apoya la enseñanza moldeadora de la conducta del estudiantado, utilizando el diseño del currículo por objetivos y predomina la evaluación cuantitativa del rendimiento académico" (p. 74).

En su momento hizo grandes aportaciones al proceso educativo; sin embargo, más adelante se comenzaron a encontrar fallas y críticas a sus resultados y métodos de enseñanza. Por ejemplo, Burrus Frederick Skinner, quien también se declaró conductista, hizo otras aportaciones en las que señalaba que la enseñanza tradicional se encontraba revestida de un cúmulo de deficiencias que dificultaban el proceso de aprendizaje del estudiantado (González, 2004).

\subsubsection{Paradigma constructivista}

Principalmente, se fundamenta en las ideas de Jean Piaget con respecto al progreso cognitivo en el aprendizaje. Este autor se centró en dos aspectos principales, el primero, relacionado con el desarrollo del pensamiento en el ser; y, el segundo, el desarrollo morfológico que experimenta. Se enfocó en los modelos biológicos extrapolándolo a las disciplinas antes mencionadas, aplicándolo al desarrollo del intelecto y a la psicología del alumnado (Saldarriaga et al., 2016).

La problemática que aborda el constructivismo es la construcción del conocimiento. Sus fundamentos epistemológicos son el constructivismo, el interaccionismo y el relativismo; el constructivismo porque parte del supuesto de que el sujeto es activo ante el entorno, tomando de este los elementos que le resultan significativos; el interaccionismo, porque el sujeto interactúa con su entorno para poder resolver los problemas que se le presentan, y el relativismo porque depende del entorno y de las necesidades del sujeto, los procesos de conocimiento se desarrollan, generando resultados específicos, para situaciones específicas (Morales-López \& Irigoyen-Coria, 2016).

Según Regader (2020), Jean Piaget se encaminó en el desarrollo psicológico de la información y en el enfoque de la teoría constructivista del desarrollo de la inteligencia, establecida por autores como Lev Vygotsky o David Ausubel, para crear la Teoría del Aprendizaje de Piaget. En la cual explica que, en el proceso de aprendizaje, se debe realizar una reorganización de las estructuras cognitivas existentes en cada momento; a partir de nuevas experiencias y saltos cualitativos el aprendiz alcanza a interiorizar nuevos conocimientos.

Desde la perspectiva pedagógica, el constructivismo ha generado muchas expectativas en el ámbito educativo y con grandes aportes y resultados; de hecho, se considera de las más reconocidas por la psicología general (Navarrete et al., 2020). 
En este sentido, Piaget muestra, que cada alumno (sujeto) es capaz de construir su propio proceso cognoscitivo (esquema) en función de su manera de pensar, y de conocer, como resultado de la interacción con el entorno (objetos de la realidad). De esta manera, incorpora la naturaleza adaptativa de la inteligencia a partir de dos conceptos claves de su teoría y que son vinculantes, la asimilación y la acomodación, buscando equilibrar el sujeto con los objetos (Piaget, 2009). Con relación a la asimilación, Piaget la conceptualiza como aquella "acción del organismo sobre los objetos que le rodea, en tanto que, esta acción dependa de las conductas anteriores referidas a los mismos objetos o a otros análogos" (Piaget, 2009, p. 18).

Con lo expuesto hasta ahora, Piaget (2009) demuestra el carácter activo (más que pasivo) que tiene el ser para asimilar, adaptarse y responder ante los hechos que se suscitan en el entorno.

Ahora bien, otro de los precursores sobresalientes en el paradigma constructivista es Lev Vygotsky, con base en sus contribuciones en el desarrollo socio-cognitivo en los primeros años de infancia de la persona; la aparición del lenguaje y la comunicación; la construcción del lenguaje escrito; $y$, otros aspectos relacionados con el aprendizaje (Carrera \& Mazzarella, 2001). En sus obras, Vygotsky expone dos conceptos fundamentales en su propuesta, los cuales están relacionados con los instrumentos y los símbolos como medios para comprender los procesos sociales; aquí, los símbolos o signos se entienden como aquellos instrumentos que sirven como mediadores para el desarrollo de la conducta. De las pocas diferencias conceptuales entre Piaget y Vygotsky; el primero, observa los signos desde una visión funcional semiótica; mientras que el segundo, lo percibe como una mediación semiótica (Vergel, 2014).

Con respecto a la investigación, el desarrollo de la conducta, se observa que la motivación representa la simbología; mientras que, la metodología de enseñanza representa el instrumento mediante el cual se puede provocar y entender los cambios sociales que se suscitan en el aula y fuera de ella, buscando el aprendizaje significativo de la asignatura de religión.

Es por ello, que las teorías de Vygotsky se centran en el pensamiento, el lenguaje, la memoria y el juego en los procesos educativos, y en el desarrollo mental del estudiantado (Atlantic Internacional University, 2008). En virtud de las concepciones sociales que atribuye Lev Vygotsky al proceso de educación, se establece el enfoque del constructivista social, donde se considera a la persona, como el resultado de un proceso histórico y social, y el lenguaje desempeña un parte relevante en su desarrollo cognitivo. Sosteniendo que el conocimiento se basa en el proceso de interacción de la persona con el medioambiente, enfatizando aspectos sociales y culturales, y no solamente físicos (Payer, 2005). Es decir, Lev Vygotsky enfatiza en la importancia que tienen los factores sociales y culturales en la apropiación del conocimiento, y, especialmente, en el desarrollo de las diferentes actividades mentales del estudiantado, y que pueden ser asimilados por cuenta propia o con ayuda de otra persona (Bolaños et al., 2011).

Por último, estableciendo puntos de encuentro entre Piaget y Vygotsky, ambos coindicen en oponerse al aprendizaje asociativo o exclusivamente experimental (Severo, 2012). Dando cabida al concepto de aprendizaje significativo como una forma de asimilar activando experiencias y conocimientos previos con los que se relaciona e integra el nuevo conocimiento en un proceso que implica atribución de significados o comprensión de saberes (Rivas, 2015).

La teoría del aprendizaje significativo fue una contribución al campo educacional presentada en 1963 por David Paul Ausubel (19182008), en un momento histórico en el que la corriente conductista era imperante en su máxima aplicación. Al respecto, propuso como alternativa "un modelo de enseñanza-aprendizaje basado en el descubrimiento, que privilegiaba el activismo y postulaba que se aprende aquello que se descubre” (Rodríguez, 2011, p. 30) 
Dada su propuesta, la ofreció como una forma de aprendizaje del ser humano idónea, que permite asimilar conocimientos y permanece en el tiempo, es decir, la denominó receptiva significativa con aplicación en el aula y en el desarrollo de la cotidianidad (Ausubel, 2002).

De acuerdo con Rodríguez (2011), el proceso de aprendizaje significativo constituye un eje esencial en la enseñanza, donde el mismo alumnado tiene una participación activa, al asimilar y retener la información y contenido impartido a través de la asimilación de los conceptos de acuerdo con los conocimientos y experiencias que conforman su estructura cognitiva, permitiendo que aprendan de forma no arbitraria y sustantiva. En pocas palabras, se basa en la condición que el mismo estudiantado aprende, cuando define la importancia y significancia del contenido, a partir de lo que ya sabe.

Resumiendo lo antes planteado, el uso de la teoría del aprendizaje permite buscar y mostrar las condiciones y propiedades para la asimilación, las cuales se relacionan con modos efectivos de generar de forma reflexiva modificaciones cognitivas con estabilidad y propensas a ofrecer un significado propio a partir del entorno.

\subsection{La motivación como motor de interés por el aprendizaje signi- ficativo de la religión escolar}

Valenzuela et al. (2014) manifiestan que la motivación resulta de las expectativas que tiene el estudiantado de efectuar satisfactoriamente los deberes encomendados por los/as docentes y del valor de importancia, utilidad e interés que le dé a los mismos; implicando en el estudiantado una emoción que vale la pena el esfuerzo para realizar dicha actividad y aprender. En este orden de ideas, García (2014) asegura que la motivación se refiere a una necesidad o deseo específico que activa al organismo y dirige la conducta hasta el objetivo o la meta.

Por otro lado, la motivación, según Valenzuela et al. (2015), se trata de una fuerza que impulsa al estudiantado a realizar tareas que el docente envía como refuerzo de sus aprendizajes sobre los contenidos curriculares, pero esto, no quiere decir que se termina con la finalización de la tarea.

Considera Kazarián (2017) que la motivación es el conjunto de diversos factores psíquicos que guían el comportamiento de las personas, relacionado con una necesidad, es dirigido, intenso y sostenido. Por lo tanto, se puede entender la motivación como un impulso debido a una necesidad que tiene una persona para empezar o detener un proceso durante el cual se plantea un objetivo, se utilizan los recursos imprescindibles y se mantiene una determinada conducta debido a un estado interno que la activa, dirige y mantiene, con el propósito de lograr una meta.

En este sentido, se puede decir que existe una estrecha relación entre la motivación y el rendimiento, por tanto, "al ser la motivación una variable dinámica está sujeta a cambios en la intensidad de su relación con el rendimiento cuando interactúa con ciertas condiciones y estímulos del ambiente" (Rivera, 2014, p. 27).

En consecuencia, como expresan García y Betoret (2000), los/as docentes son pieza fundamental en el proceso motivacional del alumnado para lograr en ellos calidad en el proceso de enseñanza-aprendizaje, y, como tal, pueden aprovechar de un conjunto de indicadores para medir la motivación como lo plantea Del Campo (2017), sirviendo de herramienta con el propósito de observar si se encuentran realmente motivados o no el alumnado en el proceso de aprendizaje; estos indicadores son: rapidez de respuesta; perseverancia en el trabajo empezado; selección entre las diversas y posibles formas de realizar los trabajos encomendados; intensidad con que el estudiantado realiza la tarea encomendada; $y$, reflejo de la emoción que demuestra su cara o cuerpo al realizar la tarea.

De acuerdo con la literatura, existen dos tipos de motivación, la intrínseca y la extrínseca, que se analizan a continuación. 


\subsubsection{Motivación intrínseca}

La motivación intrínseca, según Rivera (2014), ocurre cuando la fuente de la motivación reside en el individuo y la tarea, en otras palabras, existe motivación sin la necesidad de que exista recompensa extrínseca, es decir, el estudiantado no depende de otros factores o circunstancias externas, simplemente estudia porque le gusta, en este caso, se transforma en aprendizaje sólido, mejorando el desempeño estudiantil. Así mismo, añade en que este tipo de motivación repercute en la autodeterminación del propio estudiantado, mencionando, a continuación, algunas de las características comunes entre el estudiantado que se encuentran motivados intrínsecamente: a) Hacen sus deberes por iniciativa propia; b) Investigan algo más de lo enseñado en clase aparte de los deberes; c) Aprenden las cosas de forma razonada y no de memoria; d) No se dan por vencidos frente a un fracaso; y, e) Se entusiasman por encontrar los resultados, y tener un alto rendimiento.

En resumen, para que el estudiantado esté motivado y obtenga buenos resultados académicos, necesariamente, debe tener voluntad propia para seguir investigando, esto implica que, actúe con curiosidad, competencia y autodeterminación, consiguiendo como resultado emociones positivas como la satisfacción. Es muy común, que el ser humano constantemente se esté esforzando por comprender y asimilar su entorno permitiendo sobrevivir a los constantes cambios que se enfrenta diariamente.

\subsubsection{Motivación extrínseca}

La motivación extrínseca acontece cuando se requiere de incentivos y sanciones para ejecutar las acciones o tareas encomendadas, es decir, el estudiantado depende de factores externos para llevar a cabo su aprendizaje. Este tipo de motivación se ha venido utilizando a lo largo de la historia, pero no siempre se logra el estímulo esperado, por tanto, se produce el efecto contrario, la desmotivación.

En este contexto, para evitar la presencia de desmotivación en el estudiantado, se debe generar un conjunto de acciones en las que Ibáñez (2016) sugiere: a) Soslayar comentarios negativos ante los intentos de participación; b) Evitar desempeñar la actividad docente con exceso en autoridad; c) Incorporar en la planificación trabajos colaborativos; d) Valorar positivamente los intentos de aprender del estudiantado; e) Resaltar más los aciertos que los fracasos; f) Conocer las causas del éxito o el fracaso; g) La enseñanza debe ser relevante y relacionada con la vida real del estudiantado; h) El contenido debe ser claro y asequible a la posibilidad del estudiantado; i) Estar atentos ante las manifestaciones de bajo nivel de motivación; j) Impulsar la participación con toma de decisiones; $\mathrm{y}, \mathrm{k}$ ) Implementar metodología preferiblemente alternativas que incluya temas y recursos de impacto durante la clase, evitando las situaciones extremas de máxima ansiedad o de aburrimiento, como, por ejemplo, las TIC.

\subsection{Las TIC como recurso motiva- cional para el aprendizaje signi- ficativo de la religión escolar}

Varios autores han abordado las tecnologías de la información y la comunicación desde diferentes aristas, entre ellos Aznar-Díaz et al. (2018) con la realidad virtual y Cabero-Almenara \& ValenciaOrtiz (2019) con la inclusión.

No obstante, para efectos de conceptualizar las TIC como una disciplina asociada a la informática es entendida como un medio de comunicación mediado por las tecnologías de la información y la comunicación que genera innovación en los procesos de educación, el trabajo y las relaciones interpersonales (Moreno-López et al., 2017).

Por su parte, Maldonado-Berea et al. (2019) consideran que las (TIC) intervienen directamente en la cultura de un país como un 
agente moderador e influyen en las identidades nacionales, pues modifican relaciones interpersonales, la producción del sentido, identificación cultural, ética y toda esfera de la vida humana. El acceso a estas requiere de la participación de los ciudadanos (...) para fortalecer los servicios de educación.

Ante tal envergadura los/as docentes tienen un rol fundamental para propiciar y motivar el mejor uso de estas para fomentar la enseñanza-aprendizaje de modo significativo, en el caso de esta investigación dirigida a los estudios de religión en el alumnado de la EGB. Respecto a la importancia del uso de las TIC coinciden Arévalo-Tapia (2016), Vallejos-Briceño (2017) y Perilla-Ramírez et al. (2019).

\section{Metodología}

Metodológicamente se ha desarrollado una investigación bajo el paradigma positivista de carácter no experimental, ex post facto y transversal, de naturaleza descriptiva y causal, en la cual se observa y analiza lo que ocurre en estado natural. Se utiliza un diseño transversal, puesto que se recogen datos en más de un grupo al mismo tiempo mediante las encuestas realizadas y enviadas utilizando una plataforma tecnológica denominada SurveyMonkey, en la que se indaga sobre una serie de variables que pretenden medir y descubrir las causas negativas que influyen en la motivación del estudiantado en las sesiones virtuales de la asignatura de religión.

El diseño de la investigación es documental y de campo; ya que, en primera instancia se acude a estudios desarrollados por otros investigadores a través de un proceso de recolectar; seleccionar; analizar; criticar e interpretar los mismos. Por otro lado, según Arias (2012) se considera de campo, toda vez que se aborda a los sujetos de estudios empleando la recolección de datos con la finalidad de conocer e interpretar el comportamiento de las categorías de estudio y sus relaciones sin manipular las variables ni el contexto.
Ahora bien, con respecto al tipo y nivel de profundidad del estudio, es descriptivo y explicativo-correlacional; en primer lugar, porque busca caracterizar el comportamiento de las categorías de análisis según reportan los sujetos de estudio, y, en segundo lugar, se persigue explicar las razones que revelan las causas y efectos mediante una prueba de hipótesis con un estudio correlacional a fin de lograr un nivel suficientemente profundo en el que muestra los resultados y conclusiones determinantes en el comportamiento de las categorías de análisis y los sujetos de estudio (Arias, 2012).

Con respecto a la población, se incluyen los ocho docentes de religión que representan el $100 \%$ y 876 estudiantes de EGB entre el 8vo grado hasta el 3er año de bachillerato de una institución modelo de la ciudad de Quito, en el año lectivo 2020-2021. La selección de la muestra se realizó siguiendo la fórmula para poblaciones finitas, toda vez que se conoce la cantidad de estudiantes que cursan estudios en la institución modelo para los grados y años seleccionados, obteniendo una muestra de 268 estudiantes con un $95 \%$ de confianza, un error muestral del $5 \%$ y una proporción esperada del $50 \%$. Mientras que, en el caso de los/as docentes se aplica el censo, ya que se encuesta a los ocho que imparten religión.

En cuanto a los instrumentos de recolección de datos se consideró como base los cuestionarios presentados por Moos et al. (1995), para lo cual fue necesario realizar un conjunto de ajustes para dirigir las interrogantes al campo de la religión, con el propósito de describir y comprender las causas-efectos que afectan la motivación en el estudiantado. Partiendo del instrumento propuesto por Moos et al. (1995), se adaptaron dos cuestionarios dirigidos al estudiantado y a los/as docentes en el que se incluyen aspectos relacionados con:

Factores personales y sociales (edad, género, curso, medio social familiar y cultural). 
- Factores académicos (relación docenteestudiante, método de enseñanza, ambiente escolar).

- Nivel de motivación del estudiantado.

- Datos sociodemográficos del docente.

- Nivel de uso de la tecnología según el estudiantado y los/as docentes.

- Nivel de disposición del estudiantado y del docente en el uso de la tecnología.

- Nivel de satisfacción y motivación del estudiantado en el aprendizaje y profesores/ profesoras en la enseñanza.

En efecto, se diseñó un cuestionario dirigido al estudiantado y otro a los/as docentes. Ambos, sometidos a la validación de constructo mediante el análisis factorial con el uso del paquete estadístico IBM ${ }^{\circledR}$ SPSS ${ }^{\circledR}$ versión 24. Para ello, se aplicó el método de extracción de componentes principales para generar la matriz de correlación por cada una de las categorías establecidas. De este modo se extraen aquellos factores con valores superiores a 1 . Se emplea la estadística descriptiva, como medida de tendencia central la media aritmética $(\dot{\mathrm{x}})$ para conocer la incidencia del ambiente de aprendizaje y el método de enseñanza de la asignatura de religión en la motivación del estudiantado; nivel de motivación y satisfacción del estudiantado en el aprendizaje.

Así mismo, se muestra un análisis correlacional, a través del coeficiente de Rho de Spearman, para cada una de las categorías de análisis.

Para desarrollar lo antes planteado, se concreta como hipótesis de trabajo que a mayor motivación y satisfacción del estudiantado, mayor es el aprendizaje de la religión en Educación Básica.

En este sentido se plantea como hipótesis alternativa $(\mathrm{H} 1)$ verificar si existe relación entre la motivación del estudiantado y su satisfacción con la metodología de enseñanza de la asignatura de religión y como hipótesis nula (H0) la no existencia de relación entre la motivación del estudiantado y su satisfacción con la metodología de enseñanza de la asignatura de religión.

\section{Resultados}

De los datos recabados en el proceso de investigación se pudo apreciar que:

- Entre los resultados positivos y negativos que inciden en la motivación del estudiantado, con valores promedios superiores a 3.50 se encuentran:

- Se sienten como miembros importantes del grupo.

- Trabajan juntos en equipo mediante aplicaciones virtuales.

- En ocasiones se sienten reprochados por el docente.

- Se le da mayor atención y motivación al estudiantado sobresaliente.

- Relacionados con el método de enseñanza que emplean los/as docentes y que ha identificado el estudiantado en el proceso formativo de la religión, el desarrollo de habilidades en los aprendices tiene un promedio de 4.36 y el uso de la metodología tradicional de dictado de contenidos un 4.27.

- Sobre aspectos relacionados con el estado de ánimo, concentración; participación; deseos por ser mejor estudiante; libertad de expresión; entre otros es preciso indicar que pocos ítems muestran que exista un nivel de motivación alta; por el contrario, las media tienden a 1 que representa la categoría de totalmente en desacuerdo o algo en desacuerdo.

- El nivel de satisfacción que reporta el estudiantado en cuanto al disfrute de aprender la religión, muestra que los valores promedio superiores a 4 consideran que no es divertido recibir las clases de religión y que se aburren en las mismas, solo el valor promedio o la media de 1.99 disfrutan las clases de religión.

- El 66,4 \% del estudiantado expresa que es importante o muy importante el uso de herramientas tecnológicas en la asignatura de religión; una cuarta parte se muestra 
indiferente y el 5,6\% le da poca importancia a su utilización.

- El $75 \%$ de maestros consideran importante el uso de herramientas tecnológicas en la asignatura de religión y a un $25 \%$ le resulta indiferente.

\section{Discusión y conclusiones}

De acuerdo con los resultados obtenidos y complementando con la comparación de los valores arrojados, por ejemplo, con la variable ambiente de aprendizaje, a partir del análisis de tendencia central con la utilización de la media, los datos indican que efectivamente esta variable influye en la motivación del estudiantado, nótese que la mayor parte de los ítems apunta a la categoría algo de acuerdo y totalmente de acuerdo. Así mismo, en cuanto a la variable método de enseñanza según expone el estudiantado la media apunta a que los/as docentes están en la búsqueda que ellos logren desarrollar un conjunto de habilidades y destrezas que permita aplicarlas en las sesiones virtuales. No obstante, también identifican casi totalmente de acuerdo que los/ as docentes no permiten la participación activa del alumno, puesto que, se dedican a dictar contenido de la materia, lo cual es constatado por los/as docentes al señalar que utilizan el método tradicional de clases magistrales y dictado de contenidos; asimismo, los aspectos positivos de la enseñanza que promueve un enfoque activoparticipativo tienden a 1 , es decir, algo en desacuerdo y totalmente en desacuerdo que se esté aplicando en la actualidad. Por tanto, es preciso establecer otro tipo de metodología de enseñanza que motiven al estudiantado a aprender la religión. Criterio con el cual coincide Zaninelli (2019) al considerar necesario que los/as docentes empleen estrategias de diversas modalidades de organización para lograr motivar al estudiantado (...).

Con relación a la categoría de motivación, es preciso indicar que pocos ítems muestran que exista un nivel de motivación alto; por el contrario, las media tienden a 1 que representa la escala de totalmente en desacuerdo o algo en desacuerdo. Ante tal hecho las situaciones con más desventajas motivacionales se refieren a la ausencia de ayuda y apoyo entre estudiantes para comprender la religión. De igual modo, no hay motivación en el estudiantado por participar activamente ni poseer interés por relacionarse con los/as docentes o entre ellos en el proceso formativo y no sienten libertad para dar su opinión y participar en las sesiones virtuales.

Relacionado con ello Kazarián (2017) resalta el papel que juega la motivación en el proceso de aprendizaje del estudiantado, donde el rol del profesorado o profesora es de gran importancia en la selección de tareas y actividades que ofrezcan retos a la inteligencia, la imaginación y satisfagan las necesidades comunicativas del estudiantado y proporcionen el genuino disfrute del proceso del aprendizaje.

En cuanto al análisis correlacional, a través del coeficiente de Rho de Spearman, para cada una de las categorías de análisis, dado que el valor de $\mathrm{p}$ es superior a 0,05 se concluye que se acepta la hipótesis alternativa, en otras palabras, se confirma que existe asociación entre cada una de las variables de la investigación, particularmente entre la motivación y la metodología de enseñanza.

En definitiva, con los resultados obtenidos del estudiantado se aprecia que efectivamente existe un alto nivel de desmotivación para el aprendizaje de la religión, ya que el alumnado experimenta desinterés; aburrimiento; apatía; disgusto y siente que pierde el tiempo cursando la materia debido a que no le encuentran un sentido de beneficio ni personal ni espiritual. Así mismo, se encuentran algunos puntos negativos en cuanto al comportamiento del docente y la forma de enseñar la materia al inclinarse con conductas de exclusión entre el estudiantado y diferenciación con preferencias claramente demostradas. Por último, existe disposición por parte del estudiantado en que se utilicen las TIC en el proceso de enseñanza-aprendizaje dada 
la importancia percibida, incluso como medio motivacional para atraer la atención y dedicación a la asignatura de religión, criterio que reafirma en sus investigaciones (Vallejos-Briceño, 2017; Flores-Cuevas, 2018).

Del análisis de lo antes planteado se puede resumir que:

- Los/as docentes propician con el ambiente de enseñanza la existencia de niveles de desmotivación del alumnado en el aprendizaje de la religión, dada la presencia de comportamientos que ocasiona inconvenientes en la relación docente-estudiante.

- La metodología tradicional de enseñanza que aplican los/as docentes no promueve la participación activa del estudiantado fomentando la pasividad y desinterés por aprender la materia.

- Independientemente de que los/as docentes se sienten muy motivados con su carrera profesional en el campo educativo; resulta necesario extender dicha motivación al estudiantado con distintas actividades $y$ estrategias metodológicas y un comportamiento inclusivo del estudiantado.

- Los resultados alcanzados, confirman y justifican la investigación realizada, toda vez que se trata afrontar con soluciones óptimas los factores que inciden en la desmotivación del estudiantado por aprender religión en la EGB; para ello, se concluye la necesidad de aplicar metodologías alternativas que incorporen las TIC para incrementar la motivación estudiantil, y, por ende, el rendimiento académico, lo que permite ampliar posibilidades para expandir la línea de investigación en aspectos relacionados con los ambientes de aprendizajes y métodos de enseñar para motivar al estudiantado.

\section{Referencias bibliográficas}

Arévalo-Tapia, F. R. (2016). Plataforma virtual Moodle en la enseñanza aprendizaje de religión y dignidad humana. (Tesis de grado pre- vio a la obtención del título de Maestría en Tecnologías para la Gestión y Práctica Docente). Pontificia Universidad Católica del Ecuador. Sede Esmeraldas.

Arias, F. (2012). El proyecto de investigación. Introducción a la metodología científica. Episteme, C.A.

Atlantic Internacional University (2008). Constructivismo y Vigotsky. Atlantic Internacional University.

Ausubel, D. P. (2002). Adquisición y retención del conocimiento. Una perspectiva cognitiva. Paidós.

Aznar-Díaz, I., Romero-Rodrígez, J. M., \& RodríguezGarcía, A. M. (2018). La tecnología móvil de realidad virtual en educación: una revisión del estado de la literatura científica en España. revisión del estado de la literatura científica en España. EDMETIC, Revista de Educación Mediática y TIC, 7(1), 256-274. https://doi.org/10.21071/edmetic.v7i1.10139

Bolaños, S., Delgado, A., Chamorro, M., Guerrero, M., \& Quilindo, J. (27 de febrero de 2011). Constructivismo Social-Lev Vigotsky.

https://bit.ly/3vp2uMf

Carrera, B., \& Mazzarella, C. (2001). Vygotsky: Enfoque sociocultural. Educere, 5(13), 41-44. https://bit.ly/38Eu0vk

Cabero-Almenera, J., \& Valencia-Ortiz, R. (2019). TIC para la inclusión: una mirada desde Latinoamérica. Aula Abierta, 48(2), 139-146. https://doi.org/10.17811/rifie.48.2.2019.139-146

Del Campo, F. (2017). La búsqueda de sentido como factor motivador del estudiante en clase de religión. Revista Electrónica Educare, 19(3), 1-32. https://bit.ly/38FIfQF

Flores-Cuevas, F. (2018). La formación pedagógica y el uso de las tecnologías de la información y comunicación dentro del proceso enseñanza aprendizaje como una propuesta para mejorar su actividad docente. EDMETIC, Revista de Educaicón Mediática y TIC, 7(1),151-173. https://doi.org/10.21071/edmetic.v7i1.10025

García, E. (2014). Psicología General. Grupo Editorial Patria S.A.

García, F., \& Betoret, F. (2000). Motivación, aprendizaje y rendimiento escolar. Revista española de motivación y emoción, 16(1), 55-65. https://bit.ly/3bLZG3Z

Garello, M. (19 de agosto de 2019). Biografías. 
https://bit.ly/3tfGhhV

González, A. (2004). Aportaciones de la psicología conductual a la educación. Sinéctica, (25), 15-22. https://bit.ly/3czBlgS

Holmes-Coto, C. L. (2018). El paradigma constructivista y el aprendizaje significativo. Universidad de Guayaquil. Facultad de Filosofía, Letras y Ciencias de la Educación.

Ibáñez, A. (2016). La desmotivación y el fracaso escolar, la motivación en el aprendizaje. EDUCA.

Kazarián, Y. (2017): Pobre motivación por el aprendizaje de inglés en los estudiantes de medicinaun reto presente, Revista Atlante: Cuadernos de Educación y Desarrollo (mayo 2017). https://bit.ly/3uoHlBH

Maldonado-Berea, G. A., García-González, J., \& Sampedro-Requena, B. E. (2019). El efecto de las TIC y redes sociales en estudiantes universitarios. RIED. Revista Iberoamericana de Educación a Distancia, 22(2).

https://doi.org/10.5944/ried.22.2.23178

Moos, R., Moos, B., \& Trickett, E. (1995). Escalas de clima social (FES, WES, CIES, CES). TEA ediciones.

Morales-López, H., \& Irigoyen-Coria, A. (2016). El Paradigma conductista y constructivista de la educación a través del decálogo del estudiante. Archivos en Medicina Familiar, 18(2), 27-30. https://bit.ly/3uoUMS6

Moreno-López, N. M., González-Roble, A. C., TorresGómez, A. C., \& Araya-Hernández, J. (2017). Alfabetización digital a padres de familia en el uso de las redes sociales. Alteridad. Revista de Educación, 12(1), 8-19, enero-junio.

http://doi.org/10.17163/alt.v12n1.2017.01

Núñez-Soler, N. E \& Lourdes-González, M. (2017). El formato Aula Taller y su incidencia sobre la motivación, el aprendizaje y el logro escolar de niños de nivel primario. Saberes y prácticas. Revista de Filosofía y Educación, 2, 1-15. https://bit.ly/3zZQBO8

Navarrete, J., Vásquez, A., Montero, E., \& Cantero, D. (2020). Significant learning in catholic religious education: The case of Temuco (Chile). British Journal of Religious Education, 42(1), 90-102.

https://doi.org/10.1080/01416200.2019.1628005

Payer, M. (2005). Teoría del constructivismo social de Lev Vygotsky en comparación con la teoria de Jean Piaget. UNAM.
Perilla-Ramírez, A. S., Ramírez-García, S., \& AgudeloAlzate, A. (2019). Impacto de las Tecnologías de la Información y la Comunicación (TIC) en la formación ética y religiosa de estudiantes de educación media vocacional. Revista electrónica de Educación Religiosa, Didáctica y Formación de Profesores, 9(1).

https://bit.ly/3AUHKhQ

Piaget, J. (2009). La psicología de la inteligencia. Crítica, S.L.

Regader, B. (27 de febrero de 2020). La Teoría del Aprendizaje de Jean Piaget.

https://bit.ly/3eDZFkE

Rivas, M. (2015). Procesos cognitivos y aprendizajes significativos. Comunidad de Madrid.

Rivera, G. (2014). La motivación del alumno y su relación con el rendimiento académico en los/as estudiantes de Bachillerato Técnico en Salud Comunitaria del Instituto República Federal de México de Comayagüela, M.D.C., durante el año lectivo 2013. Universidad Pedagógica Nacional Francisco Morazán.

Rodríguez, M. (2011). La teoría del aprendizaje significativo: una revisión aplicable a la escuela actual. Revista Electrònica d'Investigació $i$ Innovació Educativa $i$ Socioeducativa, 3(1), 29-50. https://bit.ly/30GaASr

Salas, F. (2002). Epistemología, educación y tecnología educativa. Educación, 26(1), 9-18.

https://doi.org/10.15517/revedu.v26i1.2873

Saldarriaga, P., Bravo, G., \& Loor, M. (2016). La teoría constructivista de Jean Piaget y su significación para la pedagogía contemporánea. Dominio de las Ciencias, 2, 127-137.

http://dx.doi.org/10.23857/dc.v2i3\%20Especial.298

Salinas, J. (2016). La enseñanza religiosa escolar en el pensamiento actual de la Comisión Episcopal de Enseñanza. Revista de Pedagogía, 54(3), 583-595. https://bit.ly/38CeTCE

Sánchez, R. (2012). Conductismo vs. Constructivismo: Sus principales aportes a la pedagogía, el diseño curricular e instruccional en el área de las ciencias naturales. Ensayos Pedagógicos, (2), 67-83. https://bit.ly/3eEQNvc

Severo, A. (2012). Teorías del aprendizaje de Piaget $y$ Vygotsky. IFD.

Valenzuela, J., Muñoz, C., Silva, I., Gómez, V., \& Precht, A. (2015). Motivación escolar: Claves para la 
formación motivacional de futuros docentes. Estudios Pedagógicos, 41(1), 351-361.

http://dx.doi.org/10.4067/S0718-07052015000100021

Valenzuela, J., Nieto, A., \& Muñoz, C. (2014). Motivación y disposiciones: enfoques alternativos para explicar el desempeño de habilidades de pensamiento crítico. Investigación educativa, 16(3), 16-32. https://bit.ly/3rMEIYx

Vallejos-Briceño, S. (2017). Propuesta didáctica para motivar el uso de las TIC dentro del proceso de enseñanza aprendizaje de los docentes de las Especialidades Técnicas del C.T.P. de Cartagena Sección Nocturna. Circuito 03, Dirección Regional Santa Cruz. Instituto
Tecnológico de Costa Rica. Escuela de Educación Técnica. Programa de Maestría en Educación Técnica

Vergel, R. (2014). El signo en Vygotsky y su vínculo con el desarrollo de los procesos psicológicos superiores. Folios, 65-76.

https://doi.org/10.17227/01234870.39folios65.76

Zaninelli, M. (2019). Estilos de aprendizaje de los estudiantes de cuarto y quinto año de educación secundaria del área de educación religiosa de las instituciones educativas estatales. Universidad Católica los Ángeles de Chimbote. 


\section{FijeRIDAD $\quad$ Fijaciones visuales y caracteres: incidencias en la 56 comprensión de textos expositivos

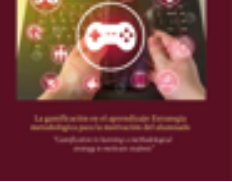

\author{
Visual fixations and characters: incidence in the understanding of \\ expository texts
}

(D) Dr. Rodolfo Antonio Padilla-Berdugo es docente de la Institución Educativa María Auxiliadora de Galapa (Colombia) (rapadilla@uninorte.edu.co) (https://orcid.org/0000-0002-5433-4626)

(1) Jorge Alberto Amador-López es docente de la Fundación Centro Educativo Mixto de Galapa (Colombia) (jamador@cemga.edu.co) (https://orcid.org/0000-0002-6173-8370)

(D) Dr. José Luis Olivo-Franco es docente de la Institución Educativa Técnica Agrícola Juan Domínguez Romero de CaracolíColombia (Colombia) (joseolivofranci@hotmail.com) (https://orcid.org/0000-0002-7781-1261)

Recibido: 2021-04-26 / Revisado: 2021-07-29 / Aceptado: 2021-11-05 / Publicado: 2022-01-01

\section{Resumen}

Por lo general, el profesorado se centra en promover la criticidad y trata, a toda costa, desarrollar procesos de intertextualidad en el estudiantado, dejando de lado los otros niveles indispensables para conseguir el producto final. En este sentido, es vital tener presente que el orden de los factores, en este caso, si alteraría el resultado, ya que velis nolis se debe llevar un ciclo y unos procedimientos específicos para lograr los objetivos trazados con antelación. Por este motivo, es necesario demostrar que las lecturas por menos y encima de lo esperado conforme a unos caracteres y cantidad de fijaciones inciden en la comprensión textual. Por otra parte, en esta investigación se aplicó un diseño transformativo secuencial con enfoque mixto, se seleccionó una muestra de 80 estudiantes de nivel secundario de una institución educativa privada del municipio de Galapa-Colombia, quienes realizaron una lectura de tres textos relacionados con el electromagnetismo en la pantalla del Eye tracker T. I 20 y con el uso del software Tobii.3.4.6. Los resultados permiten confrontar desde la práctica docente distintas posturas sobre la incursión de estas variables en los procesos lectores. Finalmente, se concluye que las fijaciones visuales cumplen un rol principal para la comprensión de un texto y por consiguiente, se plantea como prospectiva el diseño de una propuesta didáctica que tome como referente la ciencia y la imagen para buscar la comprensión adecuada por parte del lector.

Descriptores: Percepciones visuales, textos expositivos, comprensión de textos, lecturabilidad, educación básica, electromagnetismo.

\section{Abstract}

Teachers generally focus on promoting criticality and try, at all costs, to develop intertextuality processes in the student body, leaving aside the other essential levels to achieve the final product. In this sense, it is vital to bear in mind that the order of the factors, in this case, would alter the result, since velis nolis must carry out a cycle and specific procedures to achieve the objectives set in advance. For this reason, it is necessary to show that the readings for less and above than expected according to some characters and quantity of fixations affect textual comprehension. On the other hand, a transformative sequential design with a mixed approach was applied to this research, a sample of 80 students from the secondary level of a private educational institution in the municipality of Galapa-Colombia was selected, who read three texts related to the electromagnetism on the Eye tracker T.I20 screen and using Tobii.3.4.6 software. The results allow us to confront from the teaching practice different positions on the incursion of these variables in the reading processes. Finally, it is concluded that visual fixations play a main role for the understanding of a text and, therefore, the design of a didactic proposal that takes science and the image as a reference is proposed as a prospective one in order to seek adequate understanding by the student reader.

Keywords: Visual perceptions, expository texts, text comprehension, readability, basic education, electromagnetism.

Forma sugerida de citar: Padilla-Berdugo, R. A., Amador-López, J. A., \& Olivo-Franco, J. L. (2022). Fijaciones visuales y caracteres: incidencias en la comprensión de textos expositivos. Alteridad, (17)1, 126-137. https://doi.org/10.17163/alt. v17n1.2022.10 


\section{Introducción}

A pesar de que las fijaciones visuales pueden tener aplicaciones en diversas áreas (Clay et al., 2019), su incidencia en la comprensión de textos expositivos no ha sido explorada en los escolares del nivel de educación básica secundaria (EBS), específicamente en temáticas acerca del electromagnetismo (en adelante emg), caracterizado por poseer contenidos complejos y de difícil acceso, puesto que los párrafos que presentan están cargados de un discurso específico, monosémico, con un léxico estratificado y dirigido a un público en concreto; además que este debe ser analizado desde las perspectivas semánticas y pragmáticas que faciliten el acceso a la comprensión textual. Por consiguiente, se hace imprescindible tratar desde este estudio aquellos aspectos cognoscitivos relacionados con las fijaciones visuales, las características generales de los textos expositivos, su comprensión y la lecturabilidad (Jarodzka \& Brand-Gruwe, 2017).

\subsection{Las fijaciones visuales}

Una fijación visual es según Reyes (2017) "la duración de tiempo de visualización dedicado a un objetivo particular localizado en el rango foveal del ojo humano" (p. 27). Las sacadas, por otro lado, son los movimientos oculares rápidos que se presentan entre diferentes fijaciones que se evidencian frente a un objeto estímulo. Entonces, como consecuencia, el número de fijaciones produce una medida numérica que conlleva una información intrínseca para tasar, de cierta manera, el comportamiento ocular.

En este orden de ideas, se debe mencionar que según Gila et al. (2009), los ojos poseen músculos interiores que se encargan de regular el diámetro de las pupilas y la cobertura del cristalino, ya que posee movimientos impulsados a través de ciertos nervios craneales como el tercero, el cuarto y el sexto, los cuales logran que se enfoque la imagen sobre la retina; permitiendo obtener la información externa necesaria para la compren- sión. Existen tres tipos de movimientos oculares en el ser humano: los movimientos automáticos, los movimientos voluntarios de refijación, sacádicos y los micromovimientos relacionados con la fijación ocular, temblor, micro sacadas y derivadas.

Finalmente, puede decirse que según Rayner et al. (1996) el tiempo de la fijación en una lectura de textos está definido por variables como la frecuencia de vocablos, la ambigüedad del léxico, las relaciones semánticas, las anáforas, las restricciones contextuales y la complejidad sintáctica. Además, que está vinculado a variables lingüísticas que se conectan íntimamente con los movimientos oculares de cada individuo.

\subsection{Características generales del texto expositivo}

Según Alonso y Seré (1997, p. 320): "Los textos expositivos son muy difíciles de comprender muchas veces por las características que poseen". Esta tipología textual incluye en sus peculiaridades en primer lugar, una selección léxica, es decir, uso de términos monorreferenciales, de carácter endocéntricos y que se acomodan a un contenido lingüístico monosémico donde se evita el uso de sinónimos.

En segundo lugar, incorpora su propio campo terminológico, es decir que, posee una relación entre los textos excluyentes y su significado; o sea que estos conducen a la producción de nuevos documentos con léxico culto y que conllevan a una coherencia conceptual.

En tercer lugar, los textos expositivos poseen invariancia sociolectal, lo que implica el uso determinado de un vocabulario y su relación con un área específica del saber. Esta tipología textual se extiende a un lector que debe poseer unas características especiales como pertenecer a un sector social de tipo homogéneo y poseer cierto bagaje cultural en torno a las ciencias. En efecto, a mayor claridad sobre el sociolecto usado en el texto, mayor posibilidad de comprensión existirá en el individuo. 
Otra condición es la organización sintáctica o economía lingüística en el contenido textual, con vocabulario designativo y con descripciones específicas y concisas. Se debe priorizar lo científico y lo sintáctico para obtener una descripción específica; tiende más a la explicación y descripción que a la narración y argumentación; carece de cuantificadores y usa pocos calificativos por la rigurosidad de su estructura. Además, recurre a objetivos científicos precisos con propósito central.

Adicionalmente, se presenta la ausencia de elementos retóricos, es decir, la existencia de neutralidad lingüística, lo cual implica eficacia descriptiva, omisión de algunos elementos semánticos de tipo expresivo que reducen la extensión del texto, pero no su riqueza científica.

\subsection{La comprensión de textos expositivos}

Desde la perspectiva lingüística, la comprensión estaría relacionada con los procesos comunicativos, la conexión existente entre texto, lector, contexto y autor; todo visto de diferentes ángulos para lograr asimilar la intención comunicativa y facilitar el proceso de comprensión textual. Martínez et al. (2008), definen la comprensión de textos como:

Una serie de procesos cognitivos que implican formar y conectar proposiciones dentro de una frase, realizar inferencias tanto las que conectan elementos textuales como las basadas en el conocimiento y formar macro-ideas o estructuras de integración de la información. (p. 321)

Los textos expositivos según López y De León (2017, p. 270), son "aquellos que brindan información fidedigna y confiable, suministran justificaciones acerca de acontecimientos, fenómenos, tópicos; su propósito es informar y/o persuadir al lector". Estos autores también afirman que la comprensión implica un procedimiento cíclico que involucra integración y construcción de significados, además afirman que para lograr comprender se hace indispensable recorrer tres niveles de representación: primero la creación de un modelo mental en el que se parte de la superficie del texto, el procesamiento de los datos y el vínculo de estos con estructuras léxicas, jerárquicas y lingüísticas; posteriormente se crearía micro y macro estructuras, que consisten en una red proposicional de dos niveles de abstracción del significado del texto, una desde lo local y la otra desde lo global.

Por tanto, cuando se habla de comprensión textual, se relaciona con la complejidad del texto y sus características inherentes (López \& De León, 2017). De este modo, los textos expositivos requieren que el lector posea mayor compromiso atencional y conocimientos previos para acceder a la interpretación y posteriormente a la comprensión. Entonces, para comprender es indispensable implantar una correlación autorlector, por lo cual es ineludible relacionar experiencias y emociones que enlacen el contenido nuevo con los saberes previamente adquiridos.

Otro aspecto relevante es la identificación e interpretación del léxico, lo cual facilita la comprensión semántica; entonces una interpretación fallida del glosario produce una comprensión errónea. Esta tipología textual posee impersonalidad, objetividad y exactitud; características que también son propias de la ciencia y que complementadas con recursos didácticos y herramientas visuales no lingüísticas como los organizadores gráficos podrían garantizar la efectividad de la comprensión.

En este orden de ideas, un texto expositivo posee variedad de características específicas que lo hacen más complejo para su interpretación; también que su función es determinada y contextualizada desde la óptica científica.

Del mismo modo, Alonso y Séré (1997) afirman que:

Un texto expositivo posee ciertas características que los complejizan como lo son la pertenencia a un área del saber, tener un discurso con sintaxis específica y el uso determinado de la lengua.; además poseen términos léxicos o tecnolectos que complejizan la comprensión 
debido a que se hallan en un entorno académico desligado de la cotidianidad. (p. 321)

Finalmente, puede decirse que el lector es el responsable principal del proceso, puesto que su motivación a la lectura y su interpretación facilita el acceso a contenidos. El texto, por su parte, debe servir al lector como mecanismo facilitador para extraer los datos sobre funciones, clasificación, propósitos, intención comunicativa y características del documento.

\subsection{Lecturabilidad}

La lecturabilidad es un término visto desde diferentes ángulos. En primera instancia, desde lo estilístico según Sigaud-Sellos (2010) es la extensión de frases, periodicidad de expresiones, aspectos sintácticos y morfológicos que determinan la lectura adecuada y asertiva que posibilita la comprensión de textos. Por otro lado, Campos et al. (2014) afirman que "la lecturabilidad es la facilidad/dificultad con la que un texto puede ser leído y comprendido" (p. 16). Esto implica que la comprensión depende del rendimiento óptimo del lector para el proceso. La lecturabilidad no es solo la decodificación, es el dominio de recursos visuales y su asociación con documentos escritos con el propósito de permitir una comprensión instantánea (Rojas et al., 2020).

Asimismo, se debe tener en cuenta que la diversidad léxica de los textos exige al lector a tomar una actitud de motivación para conseguir la comprensión, puesto que debe asociar la mayor cantidad de palabras. Por ende, si el lector desconoce el vocabulario su comprensión será inconclusa o nula. La complejidad sintáctica, por otro lado, puede incidir en los desempeños de lectura de un individuo, ya que debe entender la estructura de las oraciones y contenido pragmático; en otras palabras, proposiciones cortas, sencillas y simples serán más fácil de interpretar que las oraciones compuestas.

En síntesis, se deduce que entre más complejidad posea el texto se hará más difícil su comprensión, ya que al lector le corresponde asociar terminologías léxicas, aspectos gramaticales y sintácticos que se relacionan pragmáticamente en un mismo contenido. En consecuencia, repercuten en el rendimiento lector y en conjunto sobre el comportamiento de los lectores frente al texto.

\section{Método}

Se aplicó un diseño transformativo secuencial, el mismo implica tener como propósito un marco de referencia conceptual transformativa, o defensa de una ideología específica, así como implementar a partir de la fase cuantitativa o la fase cualitativa; o ambos una serie de recursos en igual magnitud para conseguir ciertas metas de investigación (Cresswell et al., 2008). A partir de un diseño cuasi-experimental (Ramón, 2000) y bajo la orientación de un enfoque mixto se tomó una muestra de 80 escolares de 7 y 8 grado de nivel de educación básica secundaria pertenecientes al Centro Educativo Mixto de Galapa-Colombia, institución de naturaleza privada, ubicada en el área metropolitana de Barranquilla, para poder analizar si las lecturas de temas relacionados con el emg realizadas por encima o menos de lo esperado de acuerdo con unos caracteres y cantidad de fijaciones por párrafos inciden en la comprensión de textos expositivos.

Esta muestra fue seleccionada dentro de una población de 112 estudiantes y se atendió a unas variables control para ello, tales como contar con edades entre 12 y 13 años, no presentar déficit visual o cognitivo y no tener conocimientos previos respecto a los temas de los textos. También fue necesario aplicar una prueba de memoria operativa (Subgrupo Weschler), teniendo en cuenta que los participantes tenían que superar el límite inferior (16).

Respecto a lo cuasi-experimental, lo que se hizo al azar fue asignarles a sendos miembros de la muestra un grupo experimental por unidades de muestreo aleatorio simple y sistemático en cuatro grupos de 20 participantes A, B, C y D.

Inicialmente, los escolares leyeron individualmente en la pantalla del Eye tracker T.120 
tres lecturas relacionadas con la pila, el alternador y el timbre eléctrico después de haber observado imágenes según distintas escalas de iconicidad, luego se aplicó a cada estudiante un test de comprensión de textos expositivos (en adelante TPC) (Martínez et al., 2008), en la que nuevamente aparecieron los textos con una serie de ítems que permitió constatar si las lecturas en pantalla pudieron incidir en los resultados de la prueba realizada.

Los temas de emg seleccionados para esta investigación no hacían parte del pensum de los grados correspondientes a los participantes de la muestra. Los textos acerca de la pila, el alternador y el timbre fueron sometidos a consideración de expertos en el área cognitiva, del lenguaje y de la enseñanza de la Física. Criterios como la macroestructura, la superestructura, el modelo, la funcionalidad, la claridad, la coherencia y la relevancia se constituyeron en un referente partiendo de la reconstrucción de los textos. Para ello se tomó también un ejemplo de prueba relacionado con el texto "Los pingüinos" tomado de Martínez et al. (2008) que permitió realizar adecuaciones desde los aspectos semánticoconceptuales, la estructura de los ítems a evaluar atendiendo el nivel educativo de los escolares y las características de un texto expositivo, como se muestra en la tabla 1.

Tabla 1. Ítems del TPC

\begin{tabular}{|l|l|l|l|}
\hline \multicolumn{1}{|c|}{ Captación de ideas en una frase } & Inferencia anafórica & $\begin{array}{l}\text { Inferencia-basada en el } \\
\text { conocimiento }\end{array}$ & $\begin{array}{c}\text { Formación de } \\
\text { macro-ideas }\end{array}$ \\
\hline El & Lector & debe & \\
\hline $\begin{array}{l}\text { Descomponer la frase en proposiciones, } \\
\text { analizar las relaciones semánticas y sin- } \\
\text { tácticas que las proposiciones mantienen } \\
\text { entre sí. }\end{array}$ & $\begin{array}{l}\text { Conectar dos ideas que } \\
\text { aparecen en el texto. }\end{array}$ & $\begin{array}{l}\text { Activar esquemas previos } \\
\text { de conocimiento. }\end{array}$ & $\begin{array}{l}\text { Seleccionar lo que es } \\
\text { común con el tema } \\
\text { leído, omitiendo la in- } \\
\text { formación no esencial. }\end{array}$ \\
\hline
\end{tabular}

Fuente: Padilla (2020, p. 316$)$

Es menester recalcar que el TPC fue validado, por lo que previamente se realizó una prueba piloto a 80 escolares de la Institución educativa María Auxiliadora de Galapa-Colombia de naturaleza oficial. Fue primordial asegurar que el vocabulario, la estructura gramatical, el lenguaje y el formato de los ítems fuera el adecuado para el estudiantado; así como también se determinó el tiempo de duración por los procesos cognitivos o destrezas complejas que implican este tipo de pruebas (Medina-Díaz \& Verdejo-Carrión, 2020).

Respecto a la validación del test puede decirse que los resultados arrojaron que los escolares presentaron dificultades en tres preguntas, estas fueron sometidas a un proceso de reestructuración. Se puntuó con $0 / 1$ cada respuesta asertiva, siendo la máxima puntuación 18 puntos, pues fueron seis preguntas por tema. A partir de este pilotaje se estableció que para la aplicación del TPC con este tipo de textos se debe tener en cuenta una hora con 30 minutos, tiempo global utilizado por el grupo de participantes.

Mediante el software Tobii 3.4.6 se evaluó la CF (Cantidad de fijaciones) en los escolares haciendo uso de la técnica Eye Tracking (rastreo ocular), que tiene como propósito "registrar información de lo que el participante encontró interesante, es decir, lo que llamó su atención durante las distintas observaciones de las imágenes presentadas en el software" (Duchowski, 2007, p. 5).

\section{Resultados y análisis}

Se tuvo en cuenta el número de caracteres versus el número de fijaciones en los procesos de comprensión lectora, los cuales se convirtieron en unos criterios que permitieron con la ayuda del software Tobii 3.4.6 identificar si los escolares 
leyeron por menos de lo esperado (es decir por debajo de la cantidad de fijaciones establecida en la tabla 2), sobreleyeron (por encima de la cantidad de fijaciones establecida en la tabla 2), realizaron lecturas incompletas, no leyeron los textos relacionados con la pila, el alternador y el timbre; y cómo se convirtieron en un referente para comprobar los resultados obtenidos en el TPC.

Las fijaciones por menos de lo esperado están relacionadas con las regresiones y las que están por encima de lo esperado puede decirse que están directamente relacionadas con las refijaciones.

Según Fernández (2011) las refijaciones, reflejan la dificultad del procesamiento léxico para la palabra y las regresiones podrían también ser un indicativo de una dificultad oculomotora, donde hay un ajuste de la posición de la fijación dentro de la palabra, sin tener en cuenta el estado léxico de la palabra fijada (p.52-53).

En cambio, otras explicaciones acerca de las regresiones están relacionadas con las expuestas por Tremps-Garín (2014), pues este autor afirma que las regresiones presentes en los procesos lectores se caracterizan por dar importancia a la observación de los aspectos más distinguidos del texto; además, incluye aspectos preponderantes de lectura textual como retomar palabras, frases mal leídas, comprobar significados y realizar correcciones en fallos oculomotores.

Tabla 2. Número de caracteres y fijaciones para cada párrafo

\begin{tabular}{|c|c|c|}
\hline Temas & Número de caracteres & Número de Fijaciones o CF (Cantidad de Fijaciones) \\
\hline \multicolumn{3}{|c|}{ Pila } \\
\hline Párrafo 1 & 277 & 39,6 \\
\hline Párrafo 2 & 332 & 47,4 \\
\hline Párrafo 3 & 501 & 71,6 \\
\hline \multicolumn{3}{|c|}{ Alternador } \\
\hline Párrafo 1 & 188 & 26,9 \\
\hline Párrafo 2 & 305 & 43,6 \\
\hline Párrafo 3 & 414 & 59,1 \\
\hline \multicolumn{3}{|c|}{ Timbre } \\
\hline Párrafo 1 & 199 & 28,4 \\
\hline Párrafo 2 & 254 & 36,3 \\
\hline Párrafo 3 & 367 & 52,4 \\
\hline
\end{tabular}

\section{Fuente: Padilla (2020, p. 208)}

Tomando como referencia la tabla anterior se obtuvieron los siguientes resultados por grupo a partir de los datos ofrecidos por el software:

\section{Grupo A}

En el grupo A se identificaron tres lecturas incompletas en el párrafo 1 de la pila y 1 en el párrafo 3 del mismo texto. Asimismo, se dieron tres lecturas incompletas en el párrafo 1, 3 en el párrafo 2 y 1 en el párrafo 3 del alternador. Solamente se identificó 1 fijación por encima de lo esperado en el párrafo 1 del alternador (26,9 fijaciones). En la lectura del timbre se dio únicamente lectura incompleta en el párrafo 1 por parte de un estudiante. Fijaciones por más de lo esperado se dieron en la lectura del texto 
relacionado con el timbre en el párrafo $1(28,4$ fijaciones) y 1 en el párrafo $3(52,4)$; en el texto de la pila dos veces en el párrafo $1(39,6), 2$ en el párrafo $2(47,4)$ y 2 en el párrafo $3(71,6)$, a diferencia del alternador en la que se dio 1 fijación en el párrafo 1 (26,9 fijaciones). En el resultado del TPC fueron mayores los aciertos en los ítems relacionados con la pila y el de menos acierto fueron los del timbre. De 20 escolares miembros del grupo, 12 acertaron por encima del $50 \%$ de las 18 preguntas del test.

\section{Grupo B}

Las lecturas incompletas estuvieron presentes en el grupo B, esto se identifica en la lectura del párrafo 1 relacionado con la pila en la que dos escolares no la realizaron como lo esperado; así, sucedió una vez en el párrafo 2 y dos en el párrafo 3. No realizaron completamente la lectura del texto relacionado con el alternador cinco escolares en el párrafo 1 , dos en el párrafo 2 y dos en el párrafo 3. El texto acerca del timbre fue leído en su totalidad por todos los miembros del grupo. En cuanto a las fijaciones por encima de lo esperado, puede decirse que estas se centraron más en el timbre, 1 en el párrafo 1 (28,4 fijaciones), 1 en el párrafo 2 (36,3 fijaciones) y 4 en el párrafo 3 (52,4 fijaciones). En el resultado del TPC fueron mayores los aciertos en los ítems relacionados con la pila y el de menos aciertos fueron los del timbre. De 20 escolares 13 acertaron por encima del $50 \%$ de las 18 preguntas del test.

\section{Grupo C}

Respecto a los resultados del grupo C, seis lecturas fueron incompletas en el párrafo 1 del alternador, tres en el párrafo 3 y uno en el párrafo 2 . En relación con las fijaciones, dos por encima de las esperadas estuvieron presentes de igual forma en el párrafo 1 (39,6 fijaciones), párrafo 2 (47,4 fijaciones) y en el párrafo 3 (71,6 fijaciones) del texto relacionado con la pila. Dos fijaciones por más de lo esperado fueron identificadas en el párrafo 2 (43,6 fijaciones) del texto relacionado con el alter- nador. Al leer el texto acerca del timbre algunos escolares superaron las fijaciones por encima de lo esperado, uno en el párrafo $1(28,4)$, uno en el párrafo $2(36,3)$ y tres en el párrafo $3(52,4)$.

A pesar de la existencia de lecturas incompletas, puede afirmarse que estas se dieron generalmente y en menor cantidad en los textos que enuncian el alternador, por tal razón, en los resultados del TPC los ítems evaluados arrojaron la mayor cantidad de aciertos en relación con el texto que enuncia el alternador por encima de los otros textos, siendo los ítems relacionados con la prueba del timbre los de menos aciertos. De 20 escolares 13 acertaron por encima del $50 \%$ de las 18 preguntas del test.

\section{Grupo D}

En el grupo D se dio la mayor cantidad de fijaciones por menos de lo esperado de acuerdo con la cantidad de caracteres y fijaciones para la lectura de textos en la pantalla estímulos. Fueron identificadas 2 fijaciones en el párrafo 1 de la pila (39, 6 fijaciones) y en estas mismas condiciones dos en el párrafo 2 (47,4 fijaciones) y el párrafo 3 (71,6 fijaciones). Una fijación por más de lo esperado se dio una vez en el párrafo 1 del texto relacionado con la pila. Las lecturas por menos de lo esperado también se pudieron identificar en los textos que enuncian el alternador en el párrafo 1 (26,9 fijaciones) por tres escolares, en cambio en el párrafo 2 (43,6 fijaciones) fueron tres fijaciones por más de lo esperado y tres en el párrafo 3 (59,1 fijaciones).

El texto relacionado con el timbre, tuvo una fijación por encima de lo esperado en el párrafo 3 (52,4 fijaciones) por un estudiante. Los ítems evaluados en el test de comprensión de textos expositivos (TPC) arrojaron resultados en el texto relacionado con el alternador, siendo los ítems con menos acierto en las respuestas las del texto que enuncia el timbre. En el test, 14 escolares obtuvieron respuestas acertadas por encima del $50 \%$ de las 18 preguntas del test. No se realizaron lecturas incompletas. 
Se pudo conocer después de las observaciones realizadas por los escolares en la pantalla, distintos movimientos oculares y ciertos fenó- menos relacionados con el procesamiento de la información, tal como se indica en la tabla 3 .

Tabla 3. Fenómenos relacionados con el procesamiento de la información

\begin{tabular}{|c|c|c|}
\hline Mapas de calor & Mapas de rutas & Mapas de opacidad \\
\hline 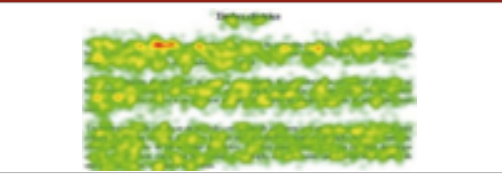 & 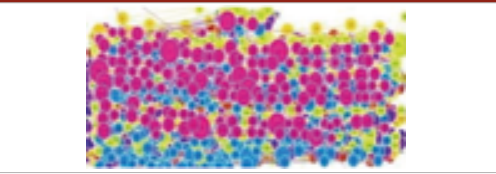 & 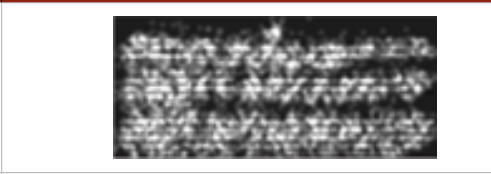 \\
\hline $\begin{array}{l}\text { Fig. Mapas de calor o heatmaps. A tra- } \\
\text { vés de ellos "se observan áreas con } \\
\text { una mayor densidad de fijaciones, mar- } \\
\text { cando con el color rojo las zonas de } \\
\text { mayor afluencia, y gradualmente varía } \\
\text { hacia tonalidades amarillas y verdes } \\
\text { según disminuye la cantidad de fijacio- } \\
\text { nes" (Martínez et al., 2015, p. } 7 \text { ). }\end{array}$ & $\begin{array}{l}\text { Fig. Gráficos de mirada o gazeplot. } \\
\text { "Cumple como función proporcionar } \\
\text { información detallada sobre el com- } \\
\text { portamiento de búsqueda del usuario, } \\
\text { tales como primeras fijaciones, la du- } \\
\text { ración de visualización y sus localiza- } \\
\text { ciones" (Reyes, } 2017, \text { p. } 27 \text { ). }\end{array}$ & $\begin{array}{l}\text { Fig. Mapas de opacidad. "Permiten } \\
\text { apreciar con más nitidez la zona que } \\
\text { ha sido más observada. Aportan la } \\
\text { misma información que los mapas } \\
\text { de calor, pero de un modo diferente" } \\
\text { (Martínez et al., 2015, p.7). }\end{array}$ \\
\hline
\end{tabular}

Fuente: Elaboración propia

\section{Discusión}

Algo que no tienen en cuenta ni los docentes de lenguaje, ni las personas vinculadas a otras disciplinas, es el hecho que las fijaciones visuales cumplen un rol principal para obtener la comprensión de un texto, ya que es a través de la vista por donde ingresa la información, que posteriormente será almacenada en el cerebro (Dharmawansa et al., 2015). Entonces todo parte del rango foveal del ojo humano, de la cantidad de fijaciones, las áreas de interés y, por ende, de la motivación del individuo para lograr la comprensión de textos. Es importante mencionar que si no hay motivación tampoco habrá fijaciones; sin ninguna de las dos tampoco habrá un proceso adecuado de lectura; y mucho menos comprensión. En este sentido, es coherente ver cómo los resultados de los mapas de calor demuestran que las fijaciones visuales se centran en las áreas de interés para el estudiantado (Cuesta-Cambra et al., 2017).

De lo anterior, surgen interrogantes como ¿qué es más importante en los procesos de lectura comprensiva de un individuo, los niveles de desempeño lector o la cantidad de fijaciones visuales que se presenten en el transcurso de la lectura?, ¿Por qué el grupo D fue el que más leyó y el que tuvo lecturas por menos de lo esperado? ¿Por qué en este grupo D no hubo sobrelectura?. Vale señalar que los procesos de lectura de adolescentes están íntimamente relacionados con diferentes variables sociales, culturales y emocionales. Por consiguiente, preguntarse sobre si un texto aborda la misma temática en términos de coherencia y cohesión global ¿Qué hace que el párrafo 3 posea mayor grado de comprensión y al mismo tiempo más lecturas por encima de lo esperado, al tiempo que ausencia de lecturas incompletas o lecturas nulas?.

En esta parte se trae a colación a Pozo (1996), quien desde la piscología piagetana examina algunas consideraciones que pueden dar luz a estos interrogantes. Así, en la perspectiva cognitiva, se dan variedad de mutaciones en cuanto a los pensamientos y operaciones formales. En otros términos, el adolescente deja de pensar y actuar como niño, comienza a pensar y concebir la realidad de un modo distinto, sus objetivos cambian, tienden a ver los contenidos 
no desde el plano del concepto o definición sino desde lo práctico.

La comprensón de un texto científico es muy compleja ya que las terminologías utilizadas son generalmente monosémicas, eluden el uso de sinónimos y tienden a ser específicas en un área de conocimiento. Lo anterior, podría ser un motivo por la cual son relevantes las refijaciones y regresiones en las lecturas (Fernández, 2011; Tremps-Garín, 2014), pues el hecho de leer menos o más respecto a una determinada cantidad de fijaciones por caracteres en los párrafos de este tipo de textos estarían más ligadas a un lenguaje que no es tan común en el grupo escolar; así como porque tiene características muy complejas que por naturaleza impiden alcanzar mayores niveles de comprensión. Tampoco sería siempre cierto afirmar que una causa de las regresiones son los fallos oculomotores. Por consiguiente, es menester partir de conocimientos previos para posibilitar la adquisición de nuevo material sobre la temática tratada (Olivo-Franco, 2021).

Además, la motivación determina la comprensión y por consiguiente puede considerarse una actitud interna y positiva que facilita el aprendizaje (Carrillo et al., 2009; Herrera y Fraga, 2009). El lector debe tener un propósito de lectura, unas metas que le permitan estar motivado. Este debe percibir el texto desde una óptica pragmática, en la cual lo vincule con el contexto en el cual se halla. Entonces, se hace necesario reconocer el circuito comunicativo del texto, relacionarlo con el contexto inmediato $y$ cultural, tener claro los intereses e instituir los sentidos del documento objeto de estudio. De este modo, la pragmática implica análisis crítico por parte del lector; a través de ella se abriría el camino para acceder a la comprensión.

Muchas veces los maestros no tienen en cuenta el tiempo que los estudiantes deben dedicar a la lectura de textos, razón que los limita en el proceso. En la imagen, por ejemplo, existe un tiempo limitado de 18 segundos de observación y esto se evidenció cuando al realizar un pilotaje de observaciones de imágenes relacionadas con temas de electromagnetismo con tres estudiantes, se pudieron conocer distracciones en la pantalla del Eye tracker T.120 después de este tiempo estipulado (Padilla, 2020); en cambio la longitud de los párrafos en variadas oportunidades genera desmotivación y fatiga para leer. Así, los tres textos trabajados en esta investigación tenían solo tres párrafos, en los cuales el estudiantado debió seleccionar lo común con el tema leído, omitir la información no esencial y, para ello, era imprescindible el aprendizaje basado en los conocimientos previos.

Otras apreciaciones importantes se refieren al hecho de examinar si la extensión de los párrafos y los textos, en general, incidieron en el hecho de que algunos alumnos no leyeran o se diera la sobre lectura, la desmotivación, la fatiga y el cansancio ocular.

\section{Conclusiones}

La mayoría de los lectores poseen dificultades en la comprensión de textos expositivos puesto que generalmente, carecen de un lenguaje rico e idóneo que les permita acceder a un discurso coherente para describir lo observado. Las estructuras gramaticales, las percepciones visuales y los intereses de los estudiantes son los grandes influenciadores de los procesos de comprensión de lectura, pues de ellos dependen en gran medida, la falta de lectura, la sobre lectura o la lectura comprensiva. Es menester resaltar que muchas veces el tiempo dedicado a estas actividades es escaso, pues generalmente, la enseñanza-aprendizaje de la lectura se presenta como un producto finiquitado que no guarda un proceso secuencial y continuo, el cual debe estar en constante evaluación.

Otro aspecto a tener presente es la incidencia de los estímulos perceptivos de la fóvea del ojo en los textos, las áreas de interés y el entendimiento de recursos semánticos inherentes en el documento al que se enfrenta el lector; quien además, debe sentirse motivado y debe entender los términos que encuentra en el documento para que se le facilite la comprensión del mismo. 
No debería descartarse que, aunque exista un alto grado de captación y fijación visual, dichos procesos en los que se extrae y se organiza la información, también estarían influenciados por los niveles de fijación por encima de unos parámetros establecidos, por la cantidad de fijaciones visuales, la sobrelectura, las diferentes regresiones formadas durante el proceso de lectura, los estados anímicos, los intereses del lector, entre otros aspectos.

De otro lado, el ser humano posee capacidades únicas para hacer uso de la vista de manera rápida, eficaz y automática, sin que esto requiera un esfuerzo adicional (Tremps-Garín, 2014; Reyes, 2017). Estas habilidades son el resultado de la cantidad de fijaciones que fueron captadas durante la observación específica de un área de interés; estas áreas influyen, de forma significativa, en la comprensión semántica de los textos expositivos.

No obstante lo anterior, los procesos de acomodación, convergencia, divergencia y visión binocular no pierden su disposición secuencial. Esta situación se da en la lectura de textos expositivos por los movimientos sacádicos, que son rápidos e irregulares, orientan la mirada hacia estímulos visuales cuyas variaciones en número, velocidad, amplitud del texto y la motivación del lector adquieren importancia en los procesos cognitivo.

El proceso de comprensión de lectura requiere de variedad de elementos distintos a los comúnmente trabajados en las escuelas, pues se vislumbran aspectos cognitivos, metacognitivos, biológicos, emocionales y psicológicos. Es pertinente mencionar que muchas veces los textos expositivos pueden generar pautas de fijación menores por sus características intrínsecas. En contraste, esta investigación determinó a través de la prueba de rastreo ocular (Eye tracker) que un grupo mínimo de los observadores no fijó la mirada en todas las áreas de interés presentes en las imágenes y obviaron el título de cada uno de los textos en la prueba TPC; del mismo modo, algunos solo leyeron oraciones presentes en un párrafo, otros lo hicieron en uno, dos o tres párrafos; y muchos otros no leyeron absolutamente nada.

Por otra parte, las estrategias pedagógicas y didácticas que presente el texto serían útiles para facilitar la comprensión, así mismo, el interés que presente el lector. Por esta razón, los textos expositivos están dirigidos a un grupo objetivo con unas características específicas que restringen las acciones del lector.

Como se ha descrito anteriormente, se confirma que los textos expositivos poseen ciertas limitaciones para un público que no está preparado para su comprensión. Todos estos aspectos son limitantes en muchas oportunidades para lograr la comprensión textual, pero es tarea de los docentes encontrar las herramientas para orientar a los escolares a hallar los procesos adecuados para comprender lo que leen. Y no es una labor única de los docentes de lenguaje, sino un compromiso de maestros de las diferentes disciplinas y del mismo educando en su autoaprendizaje.

En consecuencia, mientras más claridad posea el lector sobre el léxico empleado en el texto, mayor probabilidad de comprensión existirá. Sin embargo, muchas veces esto no se da debido al desconocimiento de los sociolectos y tecnolectos empleados y al poco bagaje científico sobre las temáticas trabajadas. Otra dificultad que muchas veces se presenta es la existencia de una neutralidad lingüística u omisión de ciertos elementos semánticos de tipo expresivo que poseen una riqueza científica a pesar de corta extensión. En efecto, son múltiples factores que facilitarían los procesos de comprensión. La labor no es para nada sencilla, pero es una tarea en la que todos los agentes involucrados deben aportar para facilitar el proceso. Se aspira que con la implementación de estos instrumentos en grupos escolares de dos o más instituciones educativas se puedan contrastar resultados que permitan seguir comprobando hipótesis respecto a la incidencia de las fijaciones visuales y los caracteres en la comprensión de textos expositivos. 


\section{Agradecimiento}

Agradecimientos a Dr, Fernando Iriarte DíazGranados. Profesor de la Universidad del Norte de Barranquilla- Colombia.

\section{Referencias bibliográficas}

Alonso, C. L., \& Séré, A. (1997). Un hipertexto de comprensión para textos científicos. Revista de filología románica (14), 319-332.

https://bit.ly/2SXkb7o

Campos, D., Contreras, P., Riffo, B., Véliz, M., \& Reyes, A. (2014). Complejidad textual, lecturabilidad y rendimiento lector en una prueba de comprensión en escolares adolescentes. Universitas psychologica, 13(3), 1135-1146.

http://dx.doi.org/10.11144/Javeriana.UPSY13-3.ctlr

Carrillo, M., Padilla, J., Rosero, T., \& Villagómez, M. S. (2009). La motivación y el aprendizaje. Alteridad, 4(1), 20-33.

https://bit.ly/3bxR1kG

Clay, V., König, P., \& König, S. (2019). Eye Tracking in Virtual Reality. Journal of eye movement research, 12(1), 10.16910/jemr.12.1.3.

https://doi.org/10.16910/jemr.12.1.3

Cresswell, J. W., Plano, V. L., Gutmann, L., \& Hanson, W. E. (2008). Avanced Mixed Methods Research Designs. En Vicki L. Plano \& Jhon W. Cresswell (1 edition), The Mixed Methods Reader (pp. 159-196). Sage Publications.

Cuesta-Cambra, U., Niño-González, I., \& Rodríguez, J. (2017). The Cognitive Processing of an Educa tional App with Electroence phalogram and "Eye Tracking". Comunicar, 52(25), 41-50. https://bit.ly/3wnn4vK

Dharmawansa, A., Fukumura1, Y., Marasinghe, A., \& Madhuwanthi, R. (2015). Introducing and Evaluating the Behavior of Non-verbal Features in the Virtual Learning. International Education Studies, 8(6), 82-94.

https://bit.ly/2UvVKhP

Duchowski, A. T. (2007). Eye tracking methodology. Theory and practice, 328.

http://dx.doi.org/10.1007/978-3-319-57883-5.

Fernández, G. (2011). Procesamiento dinámico de la información durante la lectura: estrategias mentales en la exploración visual.
https://bit.ly/3jPXih1

Gila, L., Villanueva, A., \& Cabeza, R. (2009). Fisiopatología y técnicas de registro de los movimientos oculares. En Anales del sistema sanitario de Navarra, 32, 9-26. Gobierno de Navarra. Departamento de Salud.

https://bit.ly/3dOfRP1

Herrera, C., \& Fraga, R. (2009). Etapas del proceso pedagógico. Alteridad, 4(1), 14-19.

https://bit.ly/3pZVXHu

Jarodzka, H., \& Brand-Gruwe, S. (2017). Tracking the reading eye: towards a model of realworld reading. Journal of computer assited learning, 33 (3), 193-201.

http://dx.doi.org/10.1111/jcal.12189

López, N.A. V, \& De León, T.A. M (2017) Comprensión de textos expositivos: consideraciones teóricas y pedagógicas. Revista Internacional de Ciencias Sociales y Humanidades, Sociotam, 27(2), 261-284. https://bit.ly/2Vi9pcF

Martínez, Ó. N., Díaz, A. I. M., \& Alcocer, M. L. (2015). Evaluación del color en materiales multimedia. Una experiencia con eye tracking. Edutec. Revista Electrónica de Tecnología Educativa, (54), 317-317.

https://doi.org/10.21556/edutec.2015.54.426

Martínez, T., Vidal-Abarca, E., Sellés, P., \& Gilabert, R. (2008). Evaluación de las estrategias y procesos de comprensión: el Test de Procesos de Comprensión. Infancia y Aprendizaje, 31(3), 319-332. https://bit.ly/3hkDfG0

Medina-Díaz, M. D. R., \& Verdejo-Carrión, A. L. (2020). Validez y confiabilidad en la evaluación del aprendizaje mediante las metodologías activas. Alteridad. Revista de Educación, 15(2), 270-284.

https://bit.ly/3GzadwK

Olivo-Franco, J. (2021). Mapas conceptuales: su uso para verificar el aprendizaje significativo en estudiantes de primaria. Revista Actualidades Investigativas en Educación, 21(1), 1-31. https://doi.org 10.15517/aie.v21i1.42380

Padilla, R. (2020). Efectos de las imágenes representadas según distintas escalas de iconicidad de Abraham Moles sobre la comprensión de textos expositivos relacionados con el electromagnetismo (Disertación doctoral). Universidad del Norte de Colombia. https://bit.ly/2THJ1s4 
Pozo, I. (1996). La Psicología cognitiva y la educación científica. Investigaçoes em Ensino de Ciencias 1(2), 110-131. https://bit.ly/3AzRhuR

Ramón,G. (2000).Diseñosexperimentales.Universidad de Antioquia. https://bit.ly/3ABSYYG

Rayner, K., Sereno, S. C., \& Raney, G. E. (1996). Eye movement control in reading: a comparison of two types of models. Journal of Experimental Psychology: Human Perception and Performance, 22(5), 1188.

https://doi.org/10.1037/0096-1523.22.5.1188

Reyes, M. (2017). Registro de patrones de lectura con dispositivos de Eye Tracker de bajo coste y estudio de su aplicación para la recomendación de diagnóstico de patologías (Tesis de Licenciatura). https://bit.ly/3ytqxKP
Rojas, L. D., Ibáñez, R., Moncada, F., \& Santana, A. (2020). Géneros de conocimiento en el libro de texto escolar de lengua y comunicación: Un análisis semiautomático de su lectura. RLA. Revista de lingüística teórica y aplicada, 58 (2), 41-67. https://bit.ly/31etwLQ

Sigaud-Sellos, P. (2010). Aproximación a los conceptos de legibilidad y lecturabilidad: aplicación a la lectura de textos digitales. Universidad de Navarra.

Tremps-Garín, M. D. C. (2014). Estudio de los movimientos oculares, la comprensión lectora y su influencia en el rendimiento escolar (Tesis de Maestría) https://bit.ly/3CtihvS 



\section{ALTERIDAD}

p-ISSN: 1390-325x / e-ISSN: 1390-8642

Vol. 17, No. 1/ enero-junio 2022

\section{Normas Editoriales} (Publication guidelines)

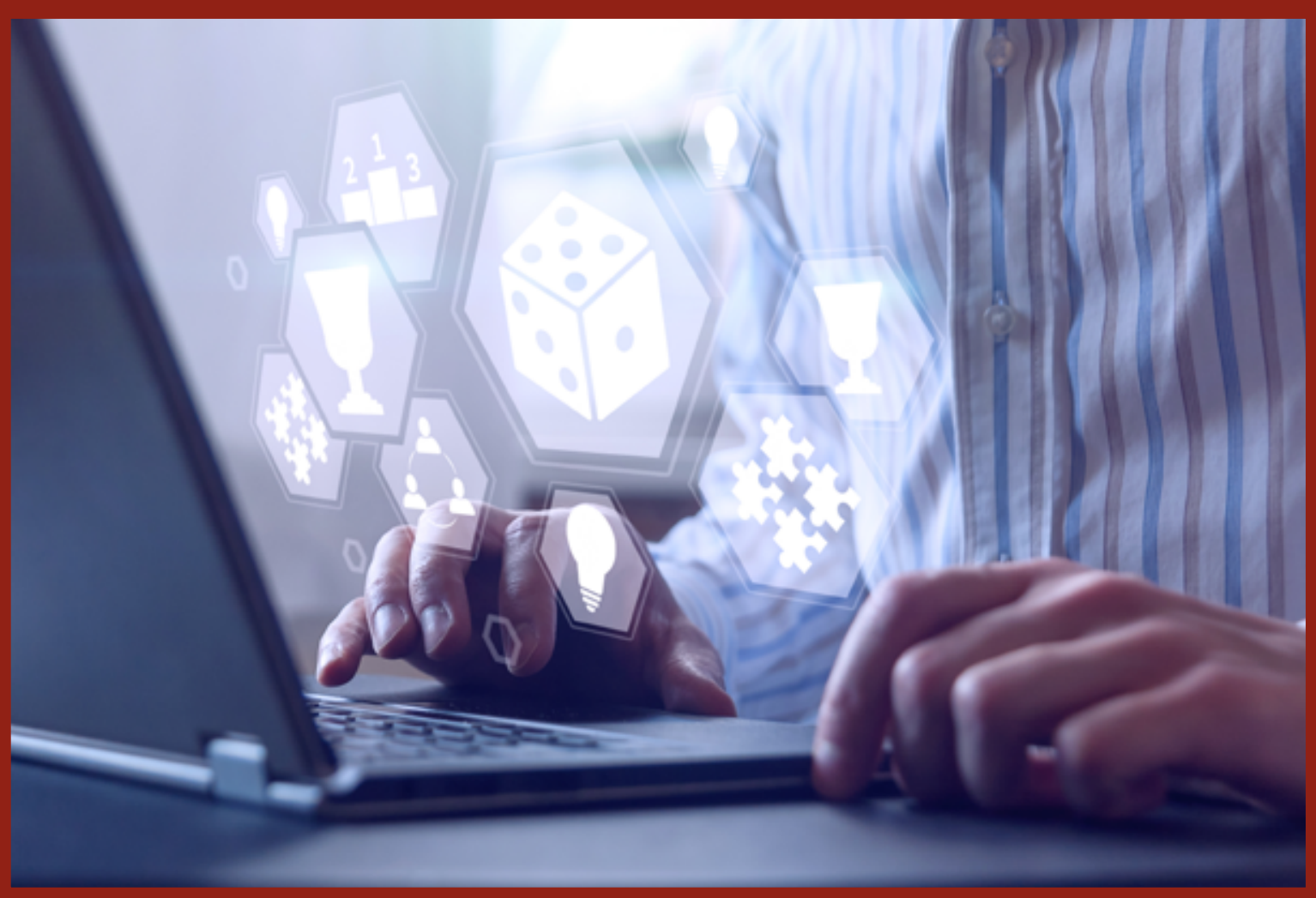




\title{
Normas de Publicación en «Alteridad»
}

\author{
http:// alteridad.ups.edu.ec/ \\ p-ISSN:1390-325X / eISSN:1390-8642
}

\section{Información general}

«Alteridad» es una publicación científica bilingüe de la Universidad Politécnica Salesiana de Ecuador (UPS), editada desde enero de 2006 de forma ininterrumpida, con periodicidad fija semestral (enero-julio).

Es una revista científica arbitrada, que utiliza el sistema de evaluación externa por expertos (peer-review), bajo metodología de pares ciegos (doble-blind review), conforme a las normas de publicación de la American Psychological Association (APA). El cumplimiento de este sistema permite garantizar a los autores un proceso de revisión objetivo, imparcial y transparente, lo que facilita a la publicación su inclusión en bases de datos, repositorios e indexaciones internacionales de referencia.

«Alteridad» se encuentra indexada en el Emerging Sources Citation Index (ESCI) de Web of Science, en la Scientific Electronic Library Online (SciELO), en el Sistema de Información Científica REDALYC, en el directorio y catálogo selectivo del Sistema Regional de Información en Línea para Revistas Científicas de América Latina, el Caribe, España y Portugal (Latindex), en el Directory of Open Access Journals (DOAJ), en el European Reference Index for the Humanities and Social Sciences (ERIHPLUS), en la Red Iberoamericana de Innovación y Conocimiento Científico (REDIB), en el Portal Dialnet; está evaluada en la Matriz de Información para el Análisis de Revistas (MIAR), en la Clasificación Integrada de Revistas Científicas (CIRC), y en el sistema Qualis de revisión de revistas de CAPES. Además, se encuentra en repositorios, bibliotecas y catálogos especializados de todo el mundo.

La revista se edita en doble versión: electrónica (e-ISSN: 1390-8642) e impresa (ISSN: 1390-325X) en español e inglés; siendo identificado cada trabajo con un Digital Object Identifier System (DOI). Todos los artículos publicados en «Alteridad» tienen licencia Creative Commons Reconocimiento-No-Comercial-Compartir igual (RoMEO blue journal).

\section{Alcance y política}

\subsection{Temática}

«Alteridad» es una revista especializada en Educación y sus líneas transdisciplinares como Didáctica, Políticas Públicas, Gerencia de Centros Escolares, Educomunicación, TIC, Pedagogía Social, entre otras; y todas aquellas disciplinas conexas interdisciplinarmente con la línea temática central.

\subsection{Secciones}

La revista tiene periodicidad semestral (20 artículos por año), publicada en los meses de enero y julio y cuenta por número con dos secciones de cinco artículos cada una, la primera referida a un tema Monográfico preparado con antelación y con editores temáticos y la segunda, una sección de Misceláneas, compuesta por aportaciones variadas dentro de la temática de la publicación. 


\subsection{Aportaciones}

Todos los trabajos deben ser originales, no haber sido publicados en ningún medio ni estar en proceso de arbitraje o publicación. Se editan preferentemente resultados de investigación empírica, redactados en español, portugués o inglés, siendo también admisibles estudios y selectas revisiones de la literatura (state-of-the-art):

- Investigaciones: 5000 a 6500 palabras de texto, incluyendo título, resúmenes, descriptores, tablas y referencias. Se valorarán especialmente los resultados de la investigación, el rigor metodológico, la relevancia de la temática, la calidad de la discusión científica, la variedad, actualidad y riqueza de las referencias bibliográficas (preferiblemente de publicaciones indexadas en JCR y Scopus). Se esperan mínimo 35 referencias.

- Estudios y revisiones de la literatura: 6000 a 7000 palabras de texto, incluidas tablas y referencias. Se valorará especialmente el debate generado, la relevancia de la temática, la originalidad de las aportaciones y referencias justificadas, actuales y selectivas de alrededor de 70 obras (preferiblemente de publicaciones indexadas en JCR y Scopus).

\section{Proceso editorial}

\subsection{Envío de manuscritos}

Los manuscritos deben ser enviados única y exclusivamente a través del Open Journal System (OJS), en el cual todos los autores deben darse de alta previamente, si bien uno solo de ellos será el responsable de correspondencia. Ningún autor podrá enviar o tener en revisión dos manuscritos de forma simultánea, estimándose una carencia de cuatro números consecutivos ( 2 años). Un artículo podrá tener como máximo 3 autores, aunque si se justifica en función del tamaño del estudio, podrán ser hasta 5.

«Alteridad» acusa recepción de los trabajos enviados por los autores, informa por email y la plataforma del proceso de aceptación o rechazo; y en el caso de aceptación, del proceso de edición.

En el Portal oficial de la revista, en la sección Normativas, están las Normas para Autores, el formato de estructura de los artículos, la Portada y Carta de presentación, el chequeo previo al envío, los formularios de evaluación por parte de los revisores externos y una guía para el envío del artículo a través de OJS. Antes de su envío se recomienda encarecidamente que se compruebe el manuscrito con el Protocolo de chequeo previo. Deben remitirse simultáneamente dos documentos:

a. Portada y Carta de presentación (usar el modelo oficial), en la que aparecerán:

- Portada (Título, Resumen y Descriptores previstos en el Manuscrito).

- Nombre y apellidos completos de cada uno de los autores, organizados por orden de prelación; seguido por la categoría profesional, centro de trabajo, correo electrónico de cada autor y número de ORCID. Es obligatorio indicar si se posee el grado académico de doctor (incluir Dr./ Dra. antes del nombre).

- Se incluirá además una declaración (Cover letter) de que el manuscrito se trata de una aportación original, no enviada ni en proceso de evaluación en otra revista, confirmación de las autorías firmantes, aceptación (si procede) de cambios formales en el manuscrito conforme a 
las normas y cesión parcial de derechos a la editorial.

b. Manuscrito totalmente anonimizado, conforme a las normas referidas en el epígrafe 4 .

\subsection{Proceso de revisión}

En un plazo máximo de 30 días, a partir de la recepción del documento, el autor de correspondencia recibirá una notificación, indicando preliminarmente si se estima o desestima para el arbitraje por los revisores científicos. En el caso de que el artículo presente deficiencias formales, no trate el tema educativo, o tenga un elevado porcentaje de similitud con otro(s) documento(s), el Consejo editorial desestimará el trabajo sin opción de vuelta. Por el contrario, si presenta carencias superficiales de forma, se devolverá al autor para su corrección antes de comenzar del proceso de evaluación. La fecha de recepción del artículo no computará hasta la recepción correcta del mismo.

Los artículos serán evaluados científicamente por una media de tres expertos en el tema. Los informes indicarán las siguientes recomendaciones: Aceptar el envío, Publicable con modificaciones, Reenviar para revisión, No publicable. A partir del análisis de los informes externos, se decidirá la aceptación o rechazo de los artículos para su publicación. En el caso de resultados discrepantes se remitirá a un nuevo dictamen, el cual será definitivo. El protocolo utilizado por los revisores es público (Investigaciones; Estudios y revisiones de la literatura).

En general, una vez vistas las revisiones científicas externas, los criterios que justifican la decisión sobre la aceptación/rechazo de los trabajos por parte del Consejo Editor son los siguientes:

- Actualidad y novedad.

- Relevancia y significación: Avance del conocimiento científico.

- Originalidad.
- Fiabilidad y validez científica: Calidad metodológica contrastada.

- Organización (coherencia lógica y presentación formal).

- Apoyos externos y financiación pública/ privada.

- Coautorías y grado de internacionalización de la propuesta y del equipo.

- Presentación: Buena redacción.

El plazo de evaluación científica de manuscritos, superados los trámites previos de estimación por el Consejo Editor, es de 100 días como máximo; los remitidos para Calls for papers, sus fechas de revisión científica se inician al cierre de los mismos. Los trabajos que sean evaluados positivamente y requieran modificaciones, deberán ser reenviados con los cambios, dentro de los siguientes 15 días.

\subsection{Edición y publicación del manuscrito}

El proceso de corrección de estilo y maquetación de los artículos aceptados es realizado por el Consejo Técnico de la Revista en coordinación con la Editorial Abya-Yala. «Alteridad» se reserva el derecho de hacer corrección de estilo y cambios editoriales que considere necesarios para mejorar el trabajo. A los autores de artículos se enviará una prueba de imprenta en formato PDF para su corrección únicamente de tipografía y ortografía en un máximo de tres días.

La Editorial Abya-Yala realizará, gratuitamente para los autores, la traducción profesional de la versión final del manuscrito al idioma inglés (o español, según la versión original), lo que garantizará su consulta y difusión internacional. Los artículos serán publicados en la plataforma de la revista en tiempo y forma. Todos los artículos, en sus dos versiones idiomáticas (español e inglés), son publicados en formato PDF, HTML, EPUB y XML-Jats. 


\subsection{Promoción y difusión del artículo publicado}

Los autores se comprometen a darle la máxima difusión a su artículo publicado en «Alteridad». En este sentido, se les exhorta a compartir y archivar su artículo publicado en las redes académicas (Academia.edu, ResearchGate, Mendeley, Kudos), sociales (Twitter, Facebook, LinkedIn, publicando en estos también el DOI), repositorios institucionales, web o blog personal, entre otras. Asimismo, se anima a los autores a compartir el artículo publicado a través de listas de correo electrónico, grupos de investigación y contactos personales.

«Alteridad» cuenta con sistemas de medición de métricas alternativas (PlumX) que permiten verificar el cumplimiento de este compromiso. Para la postulación de futuros artículos de autores de «Alteridad», se tendrá presente el impacto de los trabajos anteriores.

\section{Estructura de los manuscritos}

Los trabajos se presentarán en tipo de letra Arial 10, interlineado simple, justificado completo y sin tabuladores ni espacios en blanco entre párrafos. Solo se separarán con un espacio en blanco los grandes bloques (título, autores, resúmenes, descriptores, créditos y epígrafes). La página debe tener 2 centímetros en todos sus márgenes. Los trabajos deben presentarse en documento de Microsoft Word (.doc o .docx), siendo necesario que el archivo esté anonimizado en Propiedades de Archivo, de forma que no aparezca la identificación de autor/es.

\subsection{Portada}

Título (español) / Title (inglés): Conciso pero informativo, en castellano en primera línea y en inglés en segunda, conformado por el mayor número de términos significativos posibles. El título no solo es responsabilidad de los autores, pudiéndose proponer cambios por parte del Consejo Editorial. Se aceptan como máximo 80 caracteres con espacio.

Resumen (español) / Abstract (inglés): Se describirán de forma concisa y en este orden: Justificación del tema, objetivos, metodología empleada (enfoque y alcance), resultados más relevantes, discusión y principales conclusiones. Ha de estar escrito de manera impersonal "El presente trabajo analiza...”. En el caso del Abstract no se admitirá el empleo de traductores automáticos por su pésima calidad. Tendrá como extensión entre 220/230 palabras.

Descriptores (español) / Keywords (inglés): Se deben exponer 6 descriptores por cada versión idiomática relacionados directamente con el tema del trabajo. Será valorado positivamente el uso de las palabras claves expuestas en el Thesaurus de la UNESCO (http://bit.ly/2kIgn8I) o del Vocabulario controlado del IRESIE (http://bit.ly/2mgg4m8).

\subsection{Estructura IMRDC}

Para aquellos trabajos que se traten de Investigaciones de carácter empírico, los manuscritos respetarán rigurosamente la estructura IMRDC, siendo opcionales los epígrafes de Apoyos y Notas. Los trabajos que se traten de Estudios y revisiones de la literatura podrán ser más flexibles en sus epígrafes, especialmente en Metodología, Resultados y Discusión. En todas las tipologías de trabajos son obligatorias las Referencias bibliográficas. 
1. Introducción y estado de la cuestión: Debe incluir los fundamentos teóricos y el propósito del estudio, utilizando citas bibliográficas, así como la revisión de la literatura más significativa del tema a nivel nacional e internacional. Se valorará positivamente el uso de referencias de alto impacto (JCR y Scopus).

2. Metodología: Debe ser redactado de forma que el lector pueda comprender con facilidad el desarrollo de la investigación. Deberá contener la explicación sobre el enfoque (cuantitativo, cualitativo o mixto) y el alcance (exploratorio, descriptivo, correlacional o explicativo). En su caso, describirá la muestra y la forma de muestreo, así como se hará referencia al tipo de análisis estadístico aplicado. Si se trata de una metodología original, es necesario exponer las razones que han conducido a su empleo y describir sus posibles limitaciones.

3. Resultados: Se procurará resaltar los resultados y las observaciones más relevantes de la investigación, describiéndose, sin hacer juicios de valor, el material y métodos empleados para el análisis. Los resultados se expondrán en figuras o/y tablas según las normas de la revista (Ver epígrafe 4.4). Aparecerán en una secuencia lógica en el texto, las tablas o figuras imprescindibles, evitando la redundancia de datos.

4. Discusión y conclusiones: Resumirá los hallazgos más importantes, relacionando las propias observaciones con estudios de interés, señalando aportaciones y limitaciones, sin redundar datos ya comentados en otros apartados. Asimismo, el apartado de discusión y conclusiones debe incluir las deducciones y líneas para futuras investigaciones.

\subsection{Apoyos y Notas}

Apoyos (opcionales): El Council Science Editors recomienda a los autor/es especificar la fuente de financiación de la investigación. Se considerarán prioritarios los trabajos con aval de proyectos competitivos nacionales e internacionales. En todo caso, para la valoración científica del manuscrito, este debe ir anonimizado con XXXX solo para su evaluación inicial, a fin de no identificar autores y equipos de investigación, que deben ser explicitados en la Carta de Presentación y posteriormente en el manuscrito final.

Las notas (opcionales) irán, solo en caso necesario, al final del artículo (antes de las referencias). Deben ser utilizadas para aclarar términos o hacer anotaciones marginales. Los números de notas se colocan en superíndice, tanto en el texto como en la nota final. No se permiten notas que recojan citas bibliográficas simples (sin comentarios), pues éstas deben ir en las referencias. En caso de contener alguna cita, su referencia deberá encontrarse también en la sección de Referencias bibliográficas.

\subsection{Referencias bibliográficas}

Las citas bibliográficas deben reseñarse en forma de referencias al texto. No debe incluirse bibliografía no citada en el texto. Su número ha de ser suficiente y necesario para contextualizar el marco teórico, la metodología usada y los resultados de investigación en un espacio de investigación internacional: Mínimo 35 para los manuscritos de investigaciones de carácter empírico, y alrededor de 70 para los estudios y revisiones de literatura.

Se presentarán alfabéticamente por el apellido primero del autor (agregando el segundo solo en caso de que el primero sea de uso muy común, y unido con guion). Las citas deberán extraerse de los documentos originales preferentemente revistas y en menor medida libros. Dada la trascendencia para los índices de citas y los cálculos de los factores de impacto, se valorarán positivamente el 
uso de referencias provenientes de publicaciones indexadas en JCR y/o Scopus y la correcta citación conforme a la Norma APA 6 (http://bit.ly/2meVQcs).

Es prescriptivo que todas las citas que cuenten con DOI (Digital Object Identifier System) estén reflejadas en las Referencias (pueden obtenerse en https://search.crossref.org/). Todas las revistas y libros que no tengan DOI deben aparecer con su link (en su versión on-line, en caso de que la tengan, acortada, mediante Bitly: https://bitly.com/), y de los sitios web además la fecha de consulta en el formato indicado.

Los artículos de revistas deben ser expuestos en idioma inglés, a excepción de aquellos que se encuentren en español e inglés, caso en el que se expondrá en ambos idiomas utilizando corchetes.

\section{Normas para las referencias}

\section{a) Publicaciones periódicas}

- Artículo de revista (un autor): Ochoa, A. (2019). The type of participation promoted in schools is a constraint factor for inclusive education. [El tipo de participación que promueve la escuela, una limitante para la inclusión]. Alteridad, 14(2), 184-194. https://doi.org/10.17163/alt. v14n2.2019.03

- Artículo de revista (hasta veinte autores): Guarderas, P., Larrea, M., Cuvi, J., Vega, C., Reyes, C., Bichara, T., Ramírez, G., Paula, Ch., Pesantez, L., Íñiguez, A., Ullauri, K., Aguirre, A., Almeida, M., \& Arteaga, E. (2018). Sexual harassment in Ecuadorian universities: Content validation for instrument development. [Acoso sexual en las universidades ecuatorianas: Validez de contenido de un instrumento de medición]. Alteridad, 13(2), 214-226. https://doi.org/10.17163/alt. v13n2.2018.05

- Artículo de revista (sin DOI): López, L., \& Ramírez-García, A. (2014). Medidas disciplinarias en los centros educativos: ¿Suficientes contra el acoso escolar? Perfiles Educativos, 36(145), 32-50. https://bit.ly/37Xd5mw

\section{b) Libros y capítulos de libro}

- Libros completos: Cuéllar, J.C., \& Moncada-Paredes, M.C. (2014). El peso de la deuda externa ecuatoriana. Abya-Yala.

- Capítulos de libro: Padilla-Verdugo, J. (2014). La Historia de la Educación desde los enfoques del conocimiento. In E. Loyola (Ed.), Ciencia, Tecnología y Sociedad (CTS). Miradas desde la Educación Superior en Ecuador (pp. 107-128). Abya-Yala. https://bit.ly/3etRnZH

\section{c) Medios electrónicos}

- Aunión, J. (2011, marzo 12). La pérdida de autoridad es un problema de toda la sociedad, no es específico del aula. El País. https://bit.ly/2NIM9Dp

\section{Normas para epígrafes, tablas y figuras}

Los epígrafes del cuerpo del artículo se numerarán en arábigo. Irán sin caja completa de mayúsculas, ni subrayados, ni negritas. La numeración ha de ser como máximo de tres niveles: 1. / 1.1. / 1.1.1. Al final de cada epígrafe numerado se establecerá un retorno de carro. 
Las tablas y figuras deben presentarse incorporadas en el texto en Microsoft Word ${ }^{\circledR}$ ubicadas en el sitio en el que los autores consideren que deben estar. Se emplearán únicamente cuando sean necesarias e idóneas, debiendo limitarse su uso por cuestiones de espacios (máximo 6 entre tablas y figuras). Ambas deben ser enumeradas en arábigo y tituladas con la descripción de su contenido. Si la fuente de la tabla o figura no fuera de elaboración propia, los autores deberán incorporar al pie de la tabla o la figura la fuente de la que se extrae [por ejemplo, Fuente: Romero-Rodríguez (2016, p. 32)].

Las tablas deben estar elaboradas en el propio documento de Microsoft Word ${ }^{\circledR}$, por lo que no se aceptarán tablas cortadas y pegadas de otros documentos que no puedan ser editados en el proceso de diagramación. Las figuras, además de ser incorporadas en el documento de Microsoft Word ${ }^{\circledR}$, deberán ser enviadas como material complementario al momento del envío en el OJS de "Alteridad», debiendo tener una calidad superior a 600 dpi, en archivos de tipo TIFF, JPEG o PNG.

\section{Tasas y APC}

«Alteridad» es una revista Open Access, incluida en el Directory of Open Access Journals (DOAJ) que oferta toda su producción de forma íntegra online en abierto para toda la comunidad científica. Asimismo, no establece ninguna tasa económica durante todo el proceso editorial para la publicación de los artículos, incluyendo la revisión científica, la maquetación y la traducción de los mismos. No existe ningún publication fee, ni Article Processing Charge (APC) vinculados con esta publicación, ni para autores ni para lectores. Asimismo, la revista tiene licencia Creative-Commons ReconocimientoNo-Comercial-Compartir igual (RoMEO blue journal), lo que permite libre acceso, descarga y archivo de los artículos publicados. Todos los gastos, insumos y financiamiento de «Alteridad» provienen de los aportes realizados por la Universidad Politécnica Salesiana.

\section{Responsabilidades éticas}

Cada autor/es presentará una declaración responsable de autoría y originalidad, así como sus responsabilidades éticas contraídas.

- Originalidad: Los trabajos deben ser originales y no deben estar siendo evaluados simultáneamente en otra publicación, siendo responsabilidad de los autores el cumplimiento de esta norma. Las opiniones expresadas en los artículos publicados son responsabilidad del autor/ es. «Alteridad», como socio internacional de CrossRef ${ }^{\circledR}$, emplea la herramienta antiplagio CrossCheck ${ }^{\circledR}$ y iThenticate ${ }^{\circledR}$ para garantizar la originalidad de los manuscritos.

- Autoría: En la lista de autores firmantes deben figurar únicamente aquellas personas que han contribuido intelectualmente al desarrollo del trabajo. Haber colaborado en la recolección de datos no es, por sí mismo, criterio suficiente de autoría. «Alteridad» declina cualquier responsabilidad sobre posibles conflictos derivados de la autoría de los trabajos que se publiquen.

- Transmisión de los derechos de autor: Se incluirá en la carta de presentación la cesión de derechos del trabajo para su publicación en «Alteridad». La Universidad Politécnica Salesiana (la editorial) conserva los derechos patrimoniales (copyright) de los artículos publicados; favorece y permite la reutilización de las mismas bajo la licencia de uso indicada en ut supra. 


\title{
Publication Guidelines of «Alteridad»
}

\author{
http:// alteridad.ups.edu.ec/ \\ p-ISSN:1390-325X / eISSN:1390-86
}

\section{General information}

«Alteridad» is a bilingual scientific publication of the Salesian Polytechnic University of Ecuador (UPS), published since January 2006 uninterruptedly, on a semi-annual basis (January-July).

It is an arbitrated scientific journal, which uses the peer-review system under the double-blind review, in accordance with the publication standards of the American Psychological Association (APA). The compliance with this system ensures authors an objective, impartial and transparent review process, making it easier for authors to be included in reference international databases, repositories and indexes.

«Alteridad» is indexed in the Web of Science's Emerging Sources Citation Index (ESCI), at the Scientific Electronic Library Online (SciELO), in the REDALYC Scientific Information System, in the directory and selective catalog of the Regional Online Information System for Scientific Journals of Latin America, the Caribbean, Spain and Portugal (Latindex), in the Directory of Open Access Journals (DOAJ), in the European Reference Index for the Humanities and Social Sciences (ERIHPLUS), in the Ibero-American Network of Innovation and Scientific Knowledge (REDIB), on the Dialnet Portal; it is evaluated in the Information Matrix for Journal Analysis (MIAR), the Integrated Classification of Scientific Journals (CIRC), and the Qualis review system for CAPES journals. In addition, it is in repositories, libraries and specialized catalogs around the world.

The journal is published in two versions: electronic (e-ISSN: 1390-8642) and printed (ISSN: 1390-325X) in Spanish and English; each manuscript is identified with a Digital Object Identifier System (DOI). All articles published in «Alteridad» have the Creative Commons Attribution-NonCommercial-Share Equal license (RoMEO blue journal).

\section{Scope and policies}

\subsection{Topics}

«Alteridad» is a journal specialized in Education and its transdisciplinary lines such as Didactics, Public Policies, School Management, Edu-communication, ICT, Social Pedagogy, among others; and all those disciplines related to the main topic.

\subsection{Sections}

The journal has a semi-annual periodicity (20 articles per year), published in January and July and has two sections of five articles each by number; the first referring to a Monographic topic 
prepared in advance and with thematic topic and the second, a section of Miscellaneous, composed of varied contributions within the theme of the publication.

\subsection{Contributions}

All manuscripts must be original, and must not have been published in any other journal or must not be in the arbitration or publication process in another journal. Empirical research results are published in Spanish, Portuguese or English, and studies and state-of-the-art are also admissible:

- Researches: 5000 to 6500 text words, including title, abstracts, descriptors, tables and references. Particular assessment will be made of research results, methodological rigor, the relevance of the subject, the quality of scientific discussion, the variety, timeliness and richness of bibliographic references (preferably publications indexed in JCR and Scopus). At least 35 references are expected.

- Literature studies and reviews: 6000 to 7000 text words, including tables and references. The debate generated, the relevance of the subject, the originality, current and selective contributions and references of around 70 works (preferably from publications indexed in JCR and Scopus) will be particularly valued.

\section{Editorial process}

\subsection{Submission of manuscripts}

Manuscripts must be submitted only and exclusively through the Open Journal System (OJS), in which all authors must register in advance, although only one will be responsible for the correspondence. No author may submit or review two manuscripts simultaneously, estimating a time of four consecutive numbers ( 2 years). An article may have a maximum of 3 authors, although if justified depending on the size of the study, there may be up to 5 .

«Alteridad» informs the reception of the manuscript submitted by the authors; the information related to the acceptance or rejection of the manuscript is sent by email and the platform; and in the case of acceptance, the author is also informed of the editing process.

In the website of the journal, in the Guidelines section, are presented the Guidelines for the Authors, the format of the structure of the articles, the cover page and cover letter, the pre-submission list, the evaluation forms by the external reviewers and a guide for the submission of the article through OJS. Before the submission, it is strongly recommended that the manuscript be checked with the Pre-Check Protocol. Two documents should be sent simultaneously:

a) Cover page and cover letter (use the official model), on which will appear

- Cover page (Title, Abstract and key words provided in the Manuscript).

- Full name of each of the authors, organized in priority order; followed by the professional category, institution, email of each author and ORCID number. It is mandatory to indicate if the authors have a $\mathrm{PhD}$ academic degree (include Dr. before the name). 
- A Cover letter will also be included indicating that the manuscript is an original contribution, has not been sent or evaluated in another journal, with the signature of the authors, and acceptance (if applicable) of formal changes to the manuscript compliant with the rules and partial transfer of rights to the publisher.

b) Fully anonymized manuscript, in accordance with the rules referred to in section 4 .

\subsection{Revission process}

Upon having received the document and in a maximum period of 30 days, the correspondence author shall receive a notification, indicating whether the manuscript is estimated or dismissed for the arbitration process by the scientific reviewers. In the case that the article has formal problems, or does not address the educational subject, or has a high similarity percentage to another document(s), the editorial board shall dismiss the work without the option to return it. Conversely, if it has superficial problems, it will be returned to the author for the corrections before starting the evaluation process. The submission date of the article will be considered based on the final submission when the article is presented with the corrections.

The articles will be scientifically evaluated by an average of three experts of the topic. Reports will indicate the following recommendations: Accept the Submission, Publishable with Modifications, Sent the manuscript back for its Review, Not Publishable. The acceptance or rejection of the manuscript for its publication will be decided from the analysis of external reports. In the case of dissenting results, it shall be forwarded to a new opinion, which shall be final. The protocol used by reviewers is public (researches; studies and state-of-the-art).

In general, once the external scientific reviews are taken into view, the criteria justifying the decision on the acceptance/rejection of the manuscript by the Editorial board are:

- Current and novelty.

- Relevance and significance: Advancement of scientific knowledge.

- Originality.

- Reliability and scientific validity: Proven methodological quality.

- Organization (logical coherence and formal presentation).

- External support and public/private funding.

- Co-authoring and internationalization degree of the proposal and the team.

- Presentation: Good writing.

The timeline for the scientific evaluation of manuscripts, after the previous estimation procedures by the Editorial Board is a maximum of 100 days. In relation to the manuscripts sent for Calls for papers, their scientific review dates begin once the call finishes. Manuscripts that are positively evaluated and require modifications must be sent with the changes, within the next 15 days.

\subsection{Editing and publishing of the manuscript}

The edition and layout processes of the accepted articles is performed by the Technical Board of the journal in coordination with the Abya-Yala Editorial. «Alteridad» reserves the right to make 
style corrections and editorial changes if necessary to improve the manuscript. A proof of printing in PDF format will be sent to the authors for the correction of typography and spelling in a maximum of three days.

Abya-Yala Editorial will carry out, free of charge for the authors, the professional translation of the final version of the manuscript into the English language (or Spanish, according to the original version), which will guarantee the consultation and international dissemination of the manuscript. The articles will be published on the journal's platform in a timely manner. All articles, in their two language versions (Spanish and English), are published in PDF, HTML, EPUB and XML-Jats format.

\subsection{Promotion and dissemination of the published article}

The authors are committed to give maximum diffusion to their article published in «Alteridad». In this sense, they are encouraged to promote their published article on academic networks (Academia.edu, ResearchGate, Mendeley, Kudos), social (Twitter, Facebook, LinkedIn, also publishing the DOI), institutional repositories, web or blog staff, among others. Authors are also encouraged to share the published article through email lists, research groups and personal contacts.

«Alteridad» has a Metric Measurement System (PlumX) that allows verifying the compliance with this commitment. For the submission of future articles by authors of «Alteridad», the impact of previous works will be taken into account.

\section{Structure of the manuscripts}

The manuscripts shall be submitted in typeface Arial 10, simple spacing, fully justified and without tabs or white space between paragraphs. Only large blocks (title, authors, abstracts, key words, credits, and captions) will be separated with white space. The page must be 2 centimeters in all its margins. Manuscripts must be submitted in Microsoft Word document (.doc or .docx), requiring the file to be anonymized in File Properties to avoid the information related to the identification of the author/s.

\subsection{Cover page}

Title (Spanish and English): Concise but informative, in Spanish on the first line and in English in the second, consisting of as many significant terms as possible. The title is not only the responsibility of the authors, and changes can be proposed by the Editorial Board. A maximum of 80 characters with space are accepted.

Abstract (Spanish and English): It must be presented in a concise way and in this order: Justification, objectives, methodology used (approach and scope), more relevant results, discussion and main conclusions. It must be written impersonally "The present work analyzes.... In the case of the Abstract, the use of automatic translators will not be accepted because of their poor quality. It will be between 220/230 words.

Key words (Spanish and English): 6 keywords must be presented for each language version directly related to the topic of the manuscript. The use of the keywords presented in UNESCO's Thesaurus will be positively valued (http://bit.ly/2kIgn8I) or the controlled vocabulary of IRESIE (http://bit.ly/2mgg4m8). 


\subsection{IMRDC Structure}

For those works involving empirical research, the manuscripts will strictly respect the IMRDC structure, with the headings of Economic Supports and Notes being optional. The works involving Literature Studies and Revisions may be more flexible under their headings, especially in Methodology, Results and Discussion. In all types of works, bibliographic references are mandatory.

1. Introduction and state of the play: It should include the theoretical foundations and purpose of the study, using bibliographic citations, as well as the review of the most significant literature of the topic at the national and international level. The use of high-impact references (JCR and Scopus) will be positively valued.

2. Methodology: It must be written in a way that the reader can easily understand the development of the research. It should contain the explanation on the approach (quantitative, qualitative or mixed) and the scope (exploratory, descriptive, correlational or explanatory). When appropriate, it shall describe the sample and the sampling form, as well as it must refer to the type of statistical analysis applied. If it is an original methodology, it is necessary to set out the reasons that have led to its use and describe the possible limitations.

3. Results: Efforts will be made to highlight the most relevant results and observations of the investigation, describing, without making judgments, the material and methods used for the analysis. The results will be presented in figures or/and tables according to the journal's standards (See section 4.4). They will appear in a logical sequence in the text, tables or figures, avoiding data redundancy.

4. Discussion and conclusions: Discussion and conclusions: It will summarize the most important findings, relating the observations with interesting studies, pointing to contributions and limitations, without resulting in data already commented in other sections. In addition, the discussion and conclusions section should include deductions and lines for future research.

\subsection{Economic support and notes}

Economic support (optional): Council Science Editors recommends that authors specify the source of funding for the research. Works on the endorsement of competitive national and international projects will be considered a priority. In any case, for the scientific assessment of the manuscript, it must be anonymized with XXXX only for its initial evaluation, in order not to identify authors and research teams, which must be set out in the Presentation Letter and subsequently in the final manuscript.

Notes (optional) will go, only if necessary, at the end of the article (before references). They should be used to clarify terms or make marginal annotations. Note numbers are placed in superscript, both in the text and in the final note. Notes collecting simple bibliographic citations (without comments) are not allowed, as these should be in the references. If it contains a cite, the reference must also be found in the Bibliography section.

\subsection{Bibliography}

Bibliographical citations should be reviewed in the form of references to the text. Bibliography that is not cited should not be included in the text. Its number must be sufficient and necessary to contextualize the theoretical framework, methodology used and research results in an international research space: Minimum 35 for empirical research manuscripts, and around 70 for literature studies and reviews. 
They will be presented alphabetically by the author's first last name (adding the second one only in case the first one is very commonly used, and joined with a hyphen). The quote should be extracted from the original documents, preferably journals and to a lesser extent books. Given the significance of citation indices and impact factor calculations, the use of references from indexed publications in JCR and/or Scopus and the correct citation following APA 6 norms is valued (http:// bit.ly/2meVQcs).

It is mandatory that quotes with DOI (Digital Object Identifier System) be reflected in the References (can be obtained on https://search.crossref.org/). All journals and books without DOI must contain a link (in its online version, if applicable, and in a shorten version using Bity: https:// bitly.com/), and the websites must include the consultation date using the format provided.

Journal articles must be presented in English, with the exception of those in Spanish and English, in which case they will be presented in both languages using square brackets.

\section{Norms for the references}

\section{a) Periodic publications}

- Journal article (one author): Ochoa, A. (2019). The type of participation promoted in schools is a constraint factor for inclusive education. [El tipo de participación que promueve la escuela, una limitante para la inclusión]. Alteridad, 14(2), 184-194. https://doi.org/10.17163/alt. v14n2.2019.03

- Manuscript from a journal (until twenty authors): Guarderas, P., Larrea, M., Cuvi, J., Vega, C., Reyes, C., Bichara, T., Ramírez, G., Paula, Ch., Pesantez, L., Íñiguez, A., Ullauri, K., Aguirre, A., Almeida, M., \& Arteaga, E. (2018). Sexual harassment in Ecuadorian universities: Content validation for instrument development. [Acoso sexual en las universidades ecuatorianas: Validez de contenido de un instrumento de medición]. Alteridad, 13(2), 214-226. https://doi.org/10.17163/ alt.v13n2.2018.05

- Manuscript from a journal (without DOI): López, L., \& Ramírez-García, A. (2014). Medidas disciplinarias en los centros educativos: ¿Suficientes contra el acoso escolar? Perfiles Educativos, 36(145), 32-50. https://bit.ly/37Xd5mw.

\section{b) Books and chapters of books}

- Complete books: Cuéllar, J.C., \& Moncada-Paredes, M.C. (2014). El peso de la deuda externa ecuatoriana. Abya-Yala.

- Chapter of books: Padilla-Verdugo, J. (2014). La Historia de la Educación desde los enfoques del conocimiento. In E. Loyola (Ed.), Ciencia, Tecnología y Sociedad (CTS). Miradas desde la Educación Superior en Ecuador (pp. 107-128). Abya-Yala. https://bit.ly/3etRnZH

\section{c) Electronic means}

- Aunión, J. (2011, marzo 12). La pérdida de autoridad es un problema de toda la sociedad, no es específico del aula. $t$. https://bit.ly/2NlM9Dp 


\section{Guidelines for headings, tables and figures}

The headings of the article shall be numbered in Arabic. These will be without full case of capital letters, no underscores, no bold ones. The numbering must be at most three levels: 1. / 1.1. / 1.1.1. A carriage return will be established at the end of each numbered heading.

Tables and figures must be presented in the text in Microsoft Word ${ }^{\circledR}$ located on the place where the authors consider they should be. They shall be used only when necessary and suitable, their use should be limited for reasons of spaces (maximum 6 between tables and figures). Both must be listed in Arabic and titled with the description of their content. If the source of the table or figure is not of its own elaboration, the authors must incorporate the source consulted below the table [for example, Source: Romero-Rodríguez (2016, p. 32)].

Tables must be elaborated in Microsoft Word document, thus tables cut and pasted from other documents that cannot be edited in the diagramming process will not be accepted. The figures, in addition to being incorporated in the Microsoft Word document ${ }^{\circledR}$, must be sent as supplementary material during the submission in the «Alteridad» OJS, with a quality greater than $600 \mathrm{dpi}$, in TIFF, JPEG or PNG files.

\section{Fees and APC}

«Alteridad» is an Open Access journal, included in the Directory of Open Access Journals (DOAJ) that offers all its production online for the entire scientific community. It also does not set any economic fees throughout the editorial process for the publication of the articles, including scientific review, layout and translation thereof. There is no publication fee, no Article Processing Charge (APC) associated with this publication, neither for authors nor for readers. The journal is also licensed by Creative-Commons Attribution-Non-Commercial-Share Equal (RoMEO blue journal), which allows free access, download and archive of published articles. All expenses, inputs and financing of «Alteridad» come from the contributions made by the Salesian Polytechnic University.

\section{Ethical responsibilities}

Each author shall submit a responsible statement of authorship and originality, as well as their ethical responsibilities.

- Originality: The works must be original and should not be evaluated simultaneously in another publication, being the responsibility of the authors to comply with this standard. The opinions expressed in the published articles are the responsibility of the author/s «Alteridad» as CrossRef ${ }^{\circledR}>$ s international partner, uses the CrossCheck $^{\circledR}$ and iThenticate ${ }^{\circledR}$ anti-plagiarism tool to ensure the originality of the manuscripts.

- Authorship: The list of signatory authors should include only those who have contributed intellectually to the development of the work. Collaborating in data collection is not sufficient criteria of authorship. «Alteridad» rejects any responsibility for possible conflicts arising from the authorship of the manuscripts published.

- Transmission of copyright: the transfer of rights of the manuscript published in «Alteridad» will be included in the cover letter. The Salesian Polytechnic University (the publisher) has the copyright of published articles; it favors and allows the reuse of these under the license of use indicated above. 


\title{
ALTERIDAD
}

enero- junio 2022

ISSN $1390-325 \mathrm{X}$

e-ISSN I390-8642

\author{
CANJE \\ Se acepta canje con otras publicaciones periódicas. \\ Dirigirse a: \\ Secretaría Técnica de Comunicación \\ Universidad Politécnica Salesiana \\ Turuhuayco 3-69 y Calle Vieja \\ Casilla Postal 2074 \\ PBX: (+593 7) 2050000 - Ext. II82 \\ FAX: +593 72869112 \\ E-mail: rpublicas@ups.edu.ec \\ www.ups.edu.ec \\ Cuenca-Ecuador
}

Para canje, por favor complete el formulario a continuación y envíe a la Secretaría Técnica de Comunicación y Cultura, Universidad Politécnica Salesiana.

Nombre

Título de la publicación de canje

Institución

Dirección

Provincia o Estado

País

Teléfono

Fax

Correo electrónico

Portal electrónico 


\section{ALTERIDAD}

Volumen I7, número I (Período enero - junio 2022)

\section{Política Editorial}

«Alteridad» es una publicación científica bilingüe de la Universidad Politécnica Salesiana de Ecuador (UPS), editada desde enero de 2006 de forma ininterrumpida, con periodicidad fija semestral (enero-julio).

Es una revista científica arbitrada, que utiliza el sistema de evaluación externa por expertos (peer-review), bajo metodología de pares ciegos (doble-blind review), conforme a las normas de publicación de la American Psychological Association (APA). El cumplimiento de este sistema permite garantizar a los autores un proceso de revisión objetivo, imparcial y transparente, lo que facilita a la publicación su inclusión en bases de datos, repositorios e indexaciones internacionales de referencia.

«Alteridad» se encuentra indexada en el Emerging Sources Citation Index (ESCI) de Web of Science, en la Scientific Electronic Library Online (SciELO), en el Sistema de Información Científica REDALYC, en el directorio y catálogo selectivo del Sistema Regional de Información en Línea para Revistas Científicas de América Latina, el Caribe, España y Portugal (Latindex), en el Directory of Open Access Journals (DOAJ), en el European Reference Index for the Humanities and Social Sciences (ERIHPLUS), en la Red Iberoamericana de Innovación y Conocimiento Científico (REDIB), en el Portal Dialnet; está evaluada en la Matriz de Información para el Análisis de Revistas (MIAR), en la Clasificación Integrada de Revistas Científicas (CIRC), y en el sistema Qualis de revisión de revistas de CAPES. Además, se encuentra en repositorios, bibliotecas y catálogos especializados de todo el mundo.

La revista se edita en doble versión: electrónica (e-ISSN: I390-8642) e impresa (ISSN: I390-325X) en español e inglés; siendo identificado cada trabajo con un Digital Object Identifier System (DOI). Todos los artículos publicados en «Alteridad» tienen licencia Creative Commons Reconocimiento-No-Comercial-Compartir igual (RoMEO blue journal).
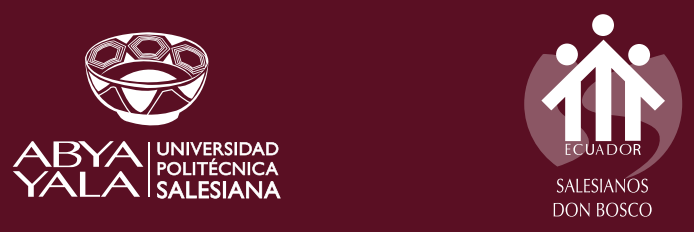